\title{
Parallele Datenakquisition zur Beschleunigung Diffusionsgewichteter Kernspintomographie mit Stimulierten Echos
}

\author{
Dissertation \\ zur Erlangung des Doktorgrades \\ der Mathematisch-Naturwissenschaftlichen Fakultäten \\ der Georg-August-Universität zu Göttingen
}

Vorgelegt von

MATTHIAS KÜNTZEL

aus Hannover

Göttingen 2006 
D7

Referent:

Korreferent:

Tag der mündlichen Prüfung:
Prof. Dr. H. Hofsäss

Prof. Dr. J. Frahm

17.08.2006 
SAPERE Aude
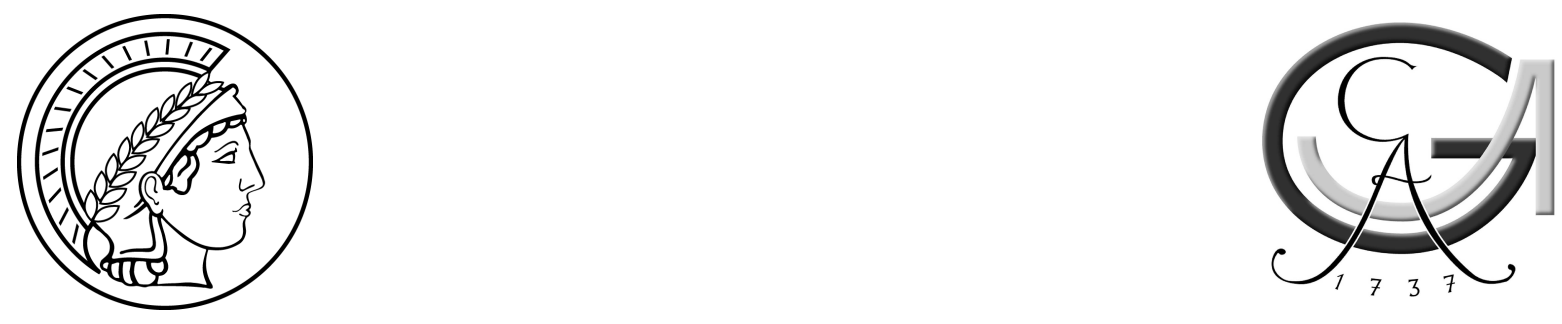



\section{Inhaltsverzeichnis}

1 Einleitung $\quad \mathbf{1}$

1.1 Eine diffusionsgewichtete Geschichte der NMR-Tomographie . . . . . . . 1

1.2 Motivation und Zielsetzung . . . . . . . . . . . . . . 2

2 Grundlagen der diffusionsgewichteten NMR-Bildgebung 4

2.1 Die Physik der Kernspinresonanz-Methode . . . . . . . . . . . . . . . 4

2.2 Makroskopische Phänomenologie der Spin-Dynamik . . . . . . . . . . 7

2.2 .1 Einfaches Spin-Echo _. . . . . . . . . . . . . 8

2.2 .2 Stimuliertes Echo . . . . . . . . . . . . . . . . . 9

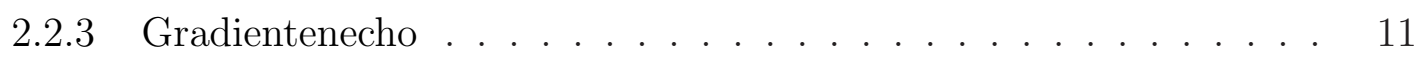

2.3 Diffusion und das NMR-Signal . . . . . . . . . . . . . . . . . . . . . . . . . . . . . . . . . . . . . . . .

2.3.1 Die Stejskal-Tanner-Sequenz . . . . . . . . . . . . . . . . 13

2.3.2 Diffusion in biologischen Strukturen . . . . . . . . . . . . . . . . . 14

2.3.3 Quantitative Beschreibung mit der Bloch-Torrey-Gleichung . . . . 16

2.3.4 Auflösung komplexer Geometrien . . . . . . . . . . . . . . . . 18

2.4 NMR-Bildgebung . . . . . . . . . . . . . . . . . . . . . . 20

2.4.1 Schichtselektive Pulse . . . . . . . . . . . . . . 20

2.4 .2 Ortskodierung . . . . . . . . . . . . . . . . . . . 21

2.4.3 Sequenzen und Bildkontrast . . . . . . . . . . . . . . . 22

2.4.4 Auflösungsvermögen . . . . . . . . . . . . . . . . 23

3 Diffusionstensorbildgebung mit DW-SSSTEAM 25

3.1 Berechnung des Diffusionstensors . . . . . . . . . . . . . . . . . . 25

3.2 Charakterisierung und Quantifizierung von DTI-Daten . . . . . . . . . 26

3.3 Artefakte, Randbedingungen und Sequenz-Konzeption . . . . . . . . . . . 30

3.3 .1 Systematische Fehler . . . . . . . . . . . . . . . . . . 30

Bildartefakte . . . . . . . . . . . . . . . 30

3.3.2 Rauschen und das SNR . . . . . . . . . . . . . . . . . 34

3.3.3 Wahl der Parameter für in-vivo Messungen . . . . . . . . . . . . 36 
3.4 Die DW-SSSTEAM Sequenz . . . . . . . . . . . . . . . . . . . 37

$3.4 .1 \quad$ Puls-Schema . . . . . . . . . . . . . . . . . . . . . . . 38

3.4.2 Die natürliche Punkt-Transfer Funktion: Wahl der Flipwinkel . . . 41

3.4 .3 k-Raum-Reduktion I: Partial Fourier . . . . . . . . . . . . . . 45

3.4.4 Referenzimplementation . . . . . . . . . . . . . . 45

3.4 .5 Versuchsaufbau . . . . . . . . . . . . . . . . . 47

4 Parallele Datenaufnahmetechniken und DW-SSSTEAM 49

4.1 k-Raum-Reduktion II: sensitivitätskodierte Bildgebung . . . . . . . . . 49

$4.1 .1 \quad$ SENSE . . . . . . . . . . . . . . . . . 50

4.1 .2 Von SMASH bis GRAPPA . . . . . . . . . . . . . 52

4.1 .3 Konsequenzen für DW-SSSTEAM . . . . . . . . . . . . . 55

4.2 Verallgemeinerung der Flipwinkelberechnung . . . . . . . . . . . 56

4.3 Theoretische Auswirkungen . . . . . . . . . . . . . . . . 58

4.3 .1 Reduktionsfaktor . . . . . . . . . . . . . . . . . . . 59

4.3 .2 Bandbreite. . . . . . . . . . . . . . . . 61

4.4 Implementierung und Einstellung der PPI-Parameter . . . . . . . . . . 62

4.5 Qualitativer Methodenvergleich . . . . . . . . . . . . . . 65

4.6 Quantifizierung der Datenqualität . . . . . . . . . . . . . 66

4.7 Diskussion . . . . . . . . . . . . . . . . . . . . . 68

5 Faserverfolgung $\quad 74$

5.1 Konventionelle Techniken . . . . . . . . . . . . . . . . . . . . 74

5.2 DTI-Traktographie . . . . . . . . . . . . . . . . . 75

5.3 Regularisierung und singuläre Punkte . . . . . . . . . . . . . . 77

$5.4 \quad \mathrm{DeffCoN} \ldots \ldots \ldots \ldots \ldots \ldots \ldots$

5.4 .1 Datenverarbeitung . . . . . . . . . . . . . 80

5.4 .2 Programm-Funktionalität . . . . . . . . . . . . . . 81

5.4 .3 Typisches Vorgehen . . . . . . . . . . . . . . . . . . . . . . . . 81

$5.5 \quad$ Ergebnisse . . . . . . . . . . . . . . . . . . . . . . . . 82

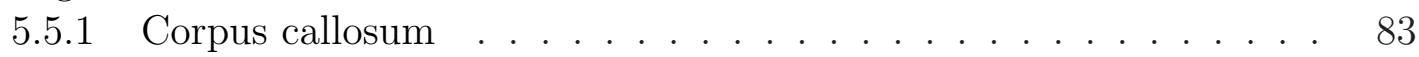

5.5 .2 Limbisches System . . . . . . . . . . . . . . . . . . 87

5.5 .3 Assoziationsbahnen . . . . . . . . . . . . . . . . . . . . . 88

5.5.4 Capsula interna (Crus anterius) . . . . . . . . . . . . . 91

5.5 .5 Hirnstamm . . . . . . . . . . . . . . . . . . . . . 9 94

$5.5 .6 \quad$ Visuelles System . . . . . . . . . . . . . . . . . . . . 97

6 Abschließende Anmerkungen 103

$\begin{array}{ll}\text { A Sequenzeinstellungen } & 109\end{array}$

$\begin{array}{ll}\text { Literaturverzeichnis } & 119\end{array}$

$\begin{array}{ll}\text { Danksagung } & 128\end{array}$ 


\section{KAPITEL 1}

\section{Einleitung}

\subsection{Eine diffusionsgewichtete Geschichte der NMR-Tomographie}

Seit seiner theoretischen und experimentellen Begründung in den Arbeiten von Rabi [1], Bloch [2, 3] und Purcell [4,5] hat das Gebiet der Kernspinresonanz-Methoden in den letzten Jahrzehnten eine stürmische Entwicklung erfahren. Die ursprüngliche Fragestellung, die Bestimmung magnetischer Momente einzelner Atomkerne, wurde sehr bald um die Untersuchung der komplexen Phänomene ihrer Wechselwirkung in kondensierter Materie erweitert. Mit der Bereicherung des experimentellen Arsenals um die von Hahn [6] eingeführten Magnetfeldpulse, ihrer Kombination zu sogenannten Sequenzen und den damit provozierten Spin-Echos erschlossen sich der wissenschaftlichen Neugierde nicht nur stationäre Zustände, wie sie durch die anfangs übliche CW-Methode (Continuous Wave) erzeugt werden, sondern auch die Spin-Dynamik sowie Möglichkeiten, diese aktiv zu manipulieren. Einen Meilenstein in der weiteren Entwicklung stellt die Verwendung von gepulsten Magnetfeldgradienten dar, die erstmals von Lauterbur [7] und Mansfield [8] in bahnbrechenden Arbeiten zur räumlichen Auflösung des NMR-Signals (Nuclear Magnetic Resonance) benutzt wurden. Sie begründeten damit das weite Feld der NMR-Tomographie, einer Disziplin, die sich neben der bestehenden Spektroskopie etablierte. Die hohen experimentellen Anforderungen machen diese Methode zu einem Kind des allgemeinen technischen Fortschritts. Ihre breite Anwendbarkeit beruht unter anderem auf der Entwicklung von supraleitenden Spulen zur Erzeugung starker, homogener Magnetfelder, dem Triumph computergestützter Meßdatenerfassung und der Entdeckung effizienter Algorithmen für die digitale Datenverarbeitung wie der schnellen Fourier-Transformation [9], FFT (Fast Fourier Transform).

Auf der Grundlage des charakteristischen Verhaltens von Kernspins in unterschiedlichen Gewebsarten und in Folge der Verfügbarkeit schneller Bildgebungssequenzen [10, 11] hat die NMR-Bildgebung insbesondere wegen ihres nichtinvasiven Charakters und der Fülle von Kontrasten die medizinische Diagnostik revolutioniert. Ihre Anwendungsgebiete reichen inzwischen von der Abbildung von Tumoren und Gewebsdefekten im Allge- 
meinen über Angiographie bis hin zur dynamischen Darstellung des schlagenden Herzens und von Aktivitätsmustern des Gehirns.

Schon früh erkannte Tanner [12], daß sich wegen des Einflusses translativer Diffusionsprozesse auf das NMR-Signal [6] die zugrunde liegende Mikrodynamik und damit auch indirekt dreidimensionale Substrukturen, z. B. die Textur von in konventionellen anatomischen Kernspintomographie-Aufnahmen relativ homogen erscheinenden Gewebsarten, charakterisieren lassen. Diese Informationen, die darüberhinaus durch Kombination mit Bildgebungssequenzen auch räumlich aufgelöst werden können, sind sowohl für medizinische Fragestellungen als auch für die Grundlagenforschung von großem Wert. Das heute übliche NMR-Verfahren zur quantitativen Bestimmung der Diffusionswirkung beruht auf der Idee von Stejskal und Tanner [13], den abschwächenden Effekt der thermischen Bewegung mittels magnetischer Gradientenpulse definiert zu verstärken. Auf dieser Basis modellierten Basser et al. [14, 15] schließlich die Richtungsabhängigkeit der Bewegung von Wassermolekülen in anisotropem Gewebe mit Hilfe eines Diffusionstensors und gaben ein Verfahren zu seiner Berechnung aus einfachen Diffusionsmessungen an. Dadurch bereiteten sie den Boden für die dreidimensionale in-vivo Darstellung des Verlaufs von Nervenfaserbündeln mit Kernspintomographie.

\subsection{Motivation und Zielsetzung}

Pulssequenzen, die diffusionsgewichtete Bildgebung realisieren, sind durch den inhärent abschwächenden Charakter der thermischen Bewegung auf das NMR-Signal, die Begleiterscheinungen der benötigten starken Gradientenfelder und nicht zuletzt durch physiologische Beschränkungen bei in-vivo Messungen mit großen technischen und methodischen Problemen behaftet. Alle Ansätze müssen daher in gewissen Bereichen Abstriche machen. Stark vereinfacht teilen sich die Verfahren in ein Lager mit vergleichsweise gutem Signal-zu-Rausch Verhältnis, SNR (Signal-to-Noise Ratio), bei geringer Meßzeit aber gleichzeitig starker Anfälligkeit gegenüber geometrischen Verzerrungen und in ein Lager konkurrierender Sequenzen mit genau konträren Eigenschaften. Da die anatomisch exakte Reproduktion eines bildgebenden Verfahrens in der medizinischen Praxis gerade auch bei intra-operativen Anwendungen eine nahezu unabdingbare Voraussetzung ist, versucht man, in dieser Hinsicht beeinträchtigte Bilder aufwendig mittels digitaler Nachverarbeitung zu korrigieren. Aufgrund des nichtaffinen Charakters der auftretenden Verzerrungen ist eine solche numerische Rekonstruktion jedoch nur eingeschränkt möglich. Darüberhinaus muß realistischerweise die anfallende Nachverarbeitungszeit bei einem denkbaren interventionellen Einsatz in der Neurochirurgie effektiv zur Datenakquisitionszeit hinzuaddiert werden. Die Alternative, das zugrunde liegende Aufnahmeverfahren unempfindlicher zu machen, führt in der Praxis häufig zu Kompromißlösungen in Bezug auf Meßzeit oder Signalstärke bei zwar abgeschwächten, aber immer noch vorhandenen topologischen Fehlern. Die angeführten Probleme lassen es deshalb vorteilhaft erscheinen, die Diffusionsbildgebung auf Akquisitionsverfahren aufzubauen, die qua Konstruktion eine geometrisch exakte Abbildung garantieren.

Die in der vorliegenden Arbeit verwendete DW-SSSTEAM-Sequenz (Diffusion-Weigh- 
ted Single-Shot Stimulated Echo Acquisition Mode) erfüllt die geforderte Bedingung, erkauft sich diesen Vorteil allerdings mit den oben erwähnten Einschränkungen im Hinblick auf Meßzeit und SNR. Um die Defizite der Methode abzumildern, wird an dieser Stelle die Möglichkeit untersucht, sie mit dem Prinzip der parallelen Bildgebung zu kombinieren, das durch Ausnutzung von Redundanzen in Aufnahmen mit Multi-SpulenEmpfängern eine reduzierte Datenakquisition ermöglicht. Dabei ist theoretisch zusätzlich zur Verkürzung der Meßzeit sogar eine moderate Zunahme des SNR zu erwarten. Das Vorhaben erfordert eine Neuprogrammierung des Auslesesegments des DW-SSSTEAMPulsprogramms, das die Form der Punkt-Transfer Funktion und damit die effektive Auflösung der resultierenden Bilder bestimmt.

Wegen des geringen Signalpegels diffusionsgewichteter Bilder und rekonstruktionsbedingter, inhomogener Verfälschungen der Bildintensitäten durch nichtlineare Transformation des Meßrauschens muß eine sorgfältige Charakterisierung des Bildmaterials und gegebenenfalls eine Anpassung der Sequenzparameter erfolgen. In einer abschließenden Bewertung der Güte des Aufnahmeverfahrens sind in letzter Konsequenz auch die Daten auf ihre Fähigkeit zu überprüfen, die untersuchten Gewebstexturen korrekt wiederzugeben. Zu diesem Zweck wurde ein umfangreiches Computerprogramm erstellt, das es in einem gewissen Rahmen erlaubt, eine solche Einschätzung vorzunehmen. Es dient in dieser Arbeit insbesondere auch dazu, Nervenfaserbündel im menschlichen Gehirn darzustellen, von denen einige in Bereichen liegen, die durch die gemeinhin verwendeten Diffusionssequenzen nur schlecht abgebildet werden. 


\section{KAPITEL 2}

\section{Grundlagen der diffusionsgewichteten NMR-Bildgebung}

\subsection{Die Physik der Kernspinresonanz-Methode}

Die von Stern und Gerlach in einem externen Magnetfeld $\boldsymbol{B}_{0}$ nachgewiesene Quantelung eines atomaren magnetischen Momentes $\boldsymbol{\mu}$ findet ihre Begründung in der Quantenmechanik durch die Diskretisierung des damit assoziierten Drehimpulses $\boldsymbol{J}$,

$$
\boldsymbol{\mu}=\gamma \boldsymbol{J}
$$

mit dem gyromagnetischen Verhältnis $\gamma$. Legt man die Quantisierungsachse $z$ in Richtung von $\boldsymbol{B}_{0}=B_{0} \boldsymbol{e}_{z}$, so hat der Operator $J_{z}$ bei gegebener Quantenzahl $j$ das Spektrum $m \hbar$, $m=-j,-j+1, \ldots,+j$, und die gleichzeitig meßbare Observable $\boldsymbol{J}^{2}$ den Eigenwert $j(j+$ 1) $\hbar^{2}$. Am Hamilton-Operator des durch Moment und Magnetfeld definierten Systems

$$
H=-\boldsymbol{\mu} \cdot \boldsymbol{B}_{0}=\gamma J_{z} B_{0}
$$

wird deutlich, daß die im magnetfeldfreien Fall entarteten Eigenzustände mit verschiedenen magnetischen Quantenzahlen $m$ durch das Magnetfeld in $2 j+1$ Niveaus aufgespalten werden (Zeeman-Effekt). Die Energielücke zwischen benachbarten Zuständen entspricht nach Planck der Übergangsfrequenz

$$
\omega_{0}=\gamma B_{0}
$$

die mit der Präzessions- oder Larmor-Frequenz des wie ein Kreisel um die $z$-Achse präzedierenden Momentes übereinstimmt.

Dieser Umstand bildet die Grundlage für ein von Rabi et al. [1] ersonnenes Experiment, bei dem Atome, deren Kerne einen Eigendrehimpuls (Kernspin) aufweisen, einem magnetischen Wechselfeld $\boldsymbol{B}_{1}$ definierter Frequenz $\omega_{1}$ ausgesetzt werden, das dem homogenen 


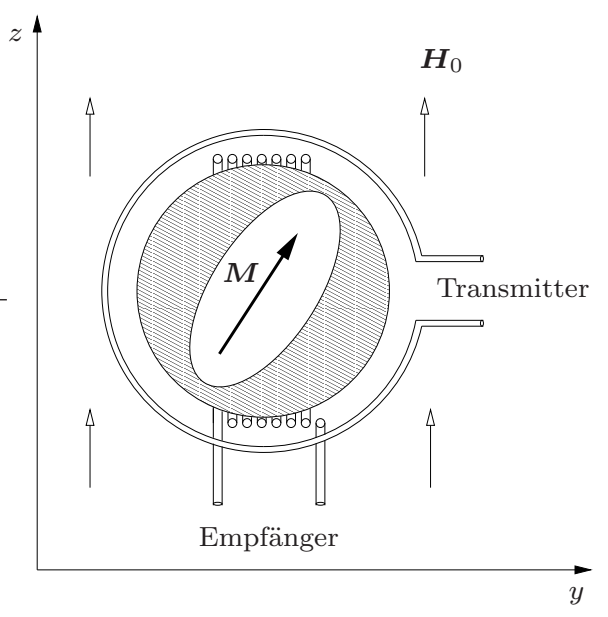

Abbildung 2.1: Schematische Darstellung eines NMR-Experiments. Die gemustert gezeichnete Probe wird von einer Empfängerspule entlang der $y$-Richtung und einer TransmitterSpule parallel zur $x$-Richtung (orthogonal zur Bildebene) umschlossen. Das externe Magnetfeld $\boldsymbol{H}_{0}$ verläuft entlang der $z$-Achse (Zeichnung nach [3]).

Magnetfeld $\boldsymbol{B}_{0}$ orthogonal überlagert ist. Die Eigenzustände des Hamilton-Operators aus Gleichung (2.2) erfahren durch die Übergangselemente des Drehimpulsoperators in dem neu hinzukommenden Störterm eine Koppelung, die bei resonanter Einstrahlung, d. h.

$$
\hbar \omega_{1}=\Delta E,
$$

durch Drehimpulsübertragung zu Übergängen zwischen jeweils zwei Eigenzuständen von $H$ mit Energiedifferenz $\Delta E$ führt. Dabei gilt die Auswahlregel $\Delta m= \pm 1$ für die korrespondierenden magnetischen Quantenzahlen. Der Resonanzfall tritt also dann auf, wenn die Anregung mit der Frequenz $\omega_{0}$ erfolgt. Weil die für Kernspins typischerweise benötigten Werte im Radiowellenbereich liegen, spricht man auch von RF-Anregung (Radiofrequenz). Bei zeitlich konstanter Einstrahlung treten durch Absorption und induzierte Emission periodische Umbesetzungen der atomaren Energiezustände auf (RabiOszillationen), die unterschiedliche magnetische Eigenschaften haben. Insbesondere war es mit solchen Verfahren möglich, die magnetischen Momente von Atomkernen mit großer Exaktheit zu vermessen, deren Verhalten in statischen und wechselnden Feldern die Grundlage der vorliegenden Arbeit bildet. In den folgenden Betrachtungen wird die Diskussion auf das für diese Dissertation relevante Zwei-Niveau-System (Spin-1/2) beschränkt, in dem die $z$-Komponente eines Spins also nur parallel oder antiparallel zum externen Magnetfeld ausgerichtet sein kann. Diese Konstellation ist in ${ }^{1} \mathrm{H}-$ Kernen realisiert, die als Bestandteil von Wasser wegen seines hohen Volumenanteils in lebendem Gewebe und ihrem großen gyromagnetischen Verhältnis primär das Signal in der NMRBildgebung bestimmen und ein endogenes Kontrastmittel darstellen.

Ein Ensemble wechselwirkungsfreier Spins in einem Magnetfeld läßt sich im Mittel durch eine makroskopische Gesamtmagnetisierung $\boldsymbol{M}$ beschreiben, da die Zeitentwicklung des quantenmechanischen Erwartungswertes $\langle\cdot\rangle$ eines atomaren Momentes $\boldsymbol{\mu}$, aus- 
gedrückt durch den Kommutator $[\cdot, \cdot]$ mit dem entsprechenden Hamilton-Operator

$$
\begin{aligned}
\frac{\mathrm{d}}{\mathrm{d} t}\langle\boldsymbol{\mu}\rangle & =\frac{\mathrm{i}}{\hbar}\langle[H, \boldsymbol{\mu}]\rangle \\
& =\gamma\langle\boldsymbol{\mu}\rangle \times \boldsymbol{B}
\end{aligned}
$$

der klassischen Bewegungsgleichung entspricht, wie man mit den Vertauschungsrelationen für Drehimpulsoperatoren zeigt. Im Gleichgewichtszustand, also inbesondere in Abwesenheit des Wechselfeldes, befindet sich ein Übermaß von Atomen im energetisch niedrigeren Zustand, wobei das Verhältnis der Besetzungszahlen $N_{+}(m=1 / 2)$ und $N_{-}(m=-1 / 2)$ bei der absoluten Temperatur $T_{\mathrm{s}}$ des Spinsystems vom Boltzmann-Faktor

$$
\frac{N_{+}}{N_{-}}=\exp \left[-\frac{\Delta E}{k_{\mathrm{B}} T_{\mathrm{s}}}\right]
$$

$\left(k_{\mathrm{B}}\right.$ ist die Boltzmann-Konstante) bestimmt wird. Weil keine Richtung in der $x$ - $y$-Ebene ausgezeichnet ist und deswegen $\left\langle\mu_{x}\right\rangle=\left\langle\mu_{y}\right\rangle=0$ gilt, hat der Vektor $\boldsymbol{M}$ nur eine nichtverschwindende $z$-Komponente $M_{z}^{0}$. Das Einschalten des zusätzlichen Strahlungsfeldes $\boldsymbol{B}_{1}$ bewirkt eine Auslenkung der Magnetisierung aus der Gleichgewichtsposition, wodurch im allgemeinen eine Transversalkomponente entsteht.

Die Übertragung der ursprünglich an Atomstrahlen erprobten Kernspinresonanz-Methode auf ausgedehnte Spin-Systeme in Festkörpern und Flüssigkeiten durch Bloch et al. [3] und Purcell et al. [4] erforderte ein theoretisches Verständnis der Relaxationsprozesse, die sich aus der Wechselwirkung eines magnetischen Moments mit der umgebenden Materie ergeben, und eine Anpassung der dynamischen Gleichungen für die Magnetisierung. Die rein phänomenologische Formulierung über die Bloch-Gleichung [2]

$$
\frac{\mathrm{d} \boldsymbol{M}}{\mathrm{d} t}=\gamma \boldsymbol{M} \times \boldsymbol{B}-\frac{M_{x} \boldsymbol{e}_{x}+M_{y} \boldsymbol{e}_{y}}{T_{2}}-\frac{\left(M_{z}-M_{z}^{0}\right)}{T_{1}} \boldsymbol{e}_{z}
$$

reicht für viele in der medizinischen NMR-Bildgebung relevante Phänomene völlig aus, obwohl sie nur in Regimen schwach gekoppelter Spinsysteme Gültigkeit hat, wie man sie z. B. in Flüssigkeiten vorfindet. Dabei beinhaltet $\boldsymbol{B}$ sowohl die Beiträge des statischen als auch des anregenden Magnetfeldes. Die Relaxation der Longitudinalkomponente $M_{z}$ in den Gleichgewichtszustand erfolgt auf einer charakteristischen Zeitskala $T_{1}$, die dem Einfluß der Interaktion einzelner Spins mit dem „Gitter", d. h. den restlichen Freiheitsgraden des Gesamtsystems, und dem damit verbundenen Energieübertrag geschuldet ist. Dagegen beschreibt die Zeitkonstante $T_{2}$ das Abklingen der Transversalmagnetisierung und subsumiert mehrere Einflüsse, wie Dipol-Dipol- und Quadrupol-Wechselwirkung oder indirekte Spin-Spin-Koppelung über die elektronischen Freiheitsgrade, die eine Beeinträchtigung des Phasengedächtnisses des Systems der kohärent angeregten, präzedierenden atomaren Momente bewirken. In Flüssigkeiten spielen speziell auch molekulare Bewegungen und die damit verbundenen Fluktuationen der Wechselwirkungsstärke eine Rolle [5]. Die chemische Abschirmung der Magnetfelder am Ort eines Kernspins durch umgebende Ladungsträger führt außerdem zu einer lokalen Modulation der Larmorfrequenz. 
Den gleichen Effekt haben durch starke Suszeptibilitätsunterschiede in heterogenen Untersuchungsobjekten hervorgerufene Magnetfeldgradienten.

Nicht nur immanente sondern auch äußere Einflüsse wie Inhomogenitäten des konstanten externen Magnetfeldes führen zu einer Verbreiterung des Frequenzspektrums, was einen progressiven Verlust der Phasenlagenkohärenz der einzelnen Spins und somit eine Verminderung der transversalen Magnetisierung zur Folge hat. Ist die genaue räumliche und zeitliche Form der Inhomogenität bekannt, z. B. beim bewußten Einsatz von Magnetfeldgradienten, die dem System eine lineare räumliche Variation der $z$-Komponente von $\boldsymbol{B}$ aufprägen, kann dieser Einfluß zu einem späteren Zeitpunkt durch Inversion der Schaltung rückgängig gemacht werden. Außerdem lassen sich die Auswirkungen von statischen Magnetfeldinhomogenitäten durch die noch zu diskutierende Technik der RF-basierten Spinechos kompensieren. Man beschreibt deshalb den durch zusätzliche, im Prinzip reversible Mechanismen weiter beschleunigten Signalabfall mit der Konstanten $T_{2}^{\star}$, mit $T_{2}^{\star} \leq T_{2}$. Der $T_{2}$-Abfall ist außerdem, wie schon das Vektorbild suggeriert, auch eng mit der Relaxation der Gesamtmagnetisierung in den Gleichgewichtszustand und auf diese Weise mit $T_{1}$ verknüpft. Es gilt $T_{2} \leq T_{1}$.

Die Messung von Kernspinresonanz-Übergängen erfolgt seit den Experimenten von Bloch normalerweise direkt über den induktiven Effekt der präzedierenden Transversalmagnetisierung in einer orthogonal zum homogenen externen Feld orientierten Spule. Der Versuchsaufbau ist in Abbildung 2.1 skizziert. Abweichend von der Methode Blochs verwendet man jedoch in der NMR-Bildgebung zur Signalerzeugung kein zeitlich konstantes magnetisches Wechselfeld, sondern eine Abfolge von im allgemeinen nicht periodischen Pulsen, deren Dauer $\tau_{\mathrm{A}}$ im Idealfall klein gegenüber der Spin-Spin-Relaxationszeit $T_{2}$ ist

$$
\tau_{\mathrm{A}} \ll T_{2}
$$

Dieser auf Hahn [6] zurückgehende Ansatz erlaubt die Beobachtung freier Relaxation in Abwesenheit des anregenden Feldes, das störend auf die Datenaufnahme wirkt. In der dadurch erzeugten Dynamik können nicht nur Gleichgewichtszustände, sondern auch ausserordentlich nützliche transiente Phänomene, sogenannte Spin-Echos, auftreten, welche durch eine geeignete Wahl der Abfolge von mehreren Anregungspulsen eingeschränkt manipulierbar sind.

\subsection{Makroskopische Phänomenologie der Spin-Dynamik}

Das hier als zirkular polarisiert angenommene exzitatorische Magnetfeld $\boldsymbol{B}_{1}$ mit seiner vorzeichenbehafteten Trägerfrequenz $\omega_{1}$ wird von der Einhüllenden $B_{1}^{\mathrm{e}}(t)$ moduliert

$$
\boldsymbol{B}_{1}(t)=B_{1}^{\mathrm{e}}(t)\left[\cos \left(\omega_{1} t+\phi\right) \boldsymbol{e}_{x}-\sin \left(\omega_{1} t+\phi\right) \boldsymbol{e}_{y}\right] .
$$

Es ist typischerweise um mehrere Größenordnungen schwächer als das statische $\boldsymbol{B}_{0}$ und wird dazu orthogonal appliziert. Zur Vereinfachung der Bewegungsgleichungen während der Anregung führt man ein rotierendes Bezugssystem $\left\{\boldsymbol{e}_{x^{\prime}}, \boldsymbol{e}_{y^{\prime}}, \boldsymbol{e}_{z^{\prime}}\right\}$ ein, das sich mit der Winkelgeschwindigkeit $\omega_{1}$ um die $\boldsymbol{e}_{z}$-Achse, die Richtung des statischen Magnetfeldes, 
dreht, wobei $\boldsymbol{e}_{z^{\prime}}=\boldsymbol{e}_{z}$ gilt. Die Phasenlage $\phi$ sei so gewählt, daß $\boldsymbol{B}_{1}$ in die $\boldsymbol{e}_{x^{\prime}}$-Richtung weist. Vernachlässigt man wegen (2.8) jegliche Relaxationseffekte, so gilt im bewegten Bezugssystem

$$
\begin{aligned}
\frac{\partial \boldsymbol{M}}{\partial t} & =\gamma \boldsymbol{M} \times\left[\left(\frac{\omega_{1}}{\gamma}+B_{0}\right) \boldsymbol{e}_{z^{\prime}}+B_{1}^{\mathrm{e}} \boldsymbol{e}_{x^{\prime}}\right] \\
& \equiv \gamma \boldsymbol{M} \times \boldsymbol{B}_{\mathrm{eff}} .
\end{aligned}
$$

Der Magnetisierungsvektor vollführt also eine Rotation auf einer Kegeloberfläche um $\boldsymbol{B}_{\text {eff }}$. Im Resonanzfall $\omega_{1}+\gamma B_{0}=0$ zeigt dieses effektive Magnetfeld in Richtung der Anregung, $\boldsymbol{e}_{x^{\prime}}$. Durch geeignete Wahl von $\tau_{\mathrm{A}}$ und $B_{1}^{\mathrm{e}}$ läßt sich $\boldsymbol{M}$ um einen beliebigen Winkel $\alpha=\int_{0}^{\tau_{\mathrm{A}}} \mathrm{d} t \gamma B_{1}^{\mathrm{e}}(t)$ gegen die $\boldsymbol{e}_{z^{\prime}}$-Achse auslenken. Der in der Empfängerspule beobachtete Spannungsabfall hat seinen Ursprung in der dann im Laborsystem präzedierenden Transversalmagnetisierung nach einem $\alpha$-Puls mit $0<\alpha<180^{\circ}$.

Wie schon im Zusammenhang mit der $T_{2}$-Relaxation diskutiert, existiert in einem Spin-Ensemble eine mehr oder weniger scharfe Verteilung von Resonanzfrequenzen, deren zugehörige Populationen $\boldsymbol{M}\left(\omega_{0}\right)$ im folgenden unter der Bezeichnung Isochromaten fungieren. Die natürliche Linienbreite des Systems wird durch die Inhomogenität des externen Magnetfeldes verfälscht, weil sich durch Spin-Dephasierung eine künstlich verkürzte transversale Relaxationszeit einstellt. Hahn entdeckte bei der Applikation mehrerer Pulse experimentell Lösungen der Bloch-Gleichung, die in den folgenden Abschnitten diskutiert werden und es ermöglichen, derartige Beeinträchtigungen auszuschließen.

Für die Berechnung des Einflusses von RF-Anregungen auf die Phasenverteilung der Isochromaten ist es nützlich, die Projektion $M_{\perp}$ der ausgelenkten Magnetisierung auf die Transversalebene im mit $\omega_{0}$ rotierten Bezugssystem als Vektor in der komplexen Ebene, $M_{\perp}^{\prime}:=M_{x^{\prime}}+\mathrm{i} M_{y^{\prime}}$, darzustellen.

\subsubsection{Einfaches Spin-Echo}

Man betrachte die Auswirkungen der Pulsfolge 90-TE/2-180 mit einem Zeitintervall $T E$ unspezifizierter Länge auf ein im Gleichgewicht befindliches Spin-System, dessen Resonanzfrequenzverteilung ein Maximum bei $\omega_{0}$ habe, auf welches auch das entlang $\boldsymbol{e}_{x^{\prime}}$ gerichtete Wechselfeld $\boldsymbol{B}_{1}$ abgestimmt sei. Dann erzeugt die erste Anregung aus der Gleichgewichtsmagnetisierung $\boldsymbol{M}^{0}=M_{z}^{0} \boldsymbol{e}_{z^{\prime}}$ einen Vektor in der $\left\{x^{\prime}-y^{\prime}\right\}$-Ebene, der auf der positiven $y^{\prime}$-Achse liegt. Während der Zeit $T E / 2$ relaxiert das System gemäß der Lösung der freien Bloch-Gleichungen

$$
\begin{aligned}
& M_{\perp}^{\prime}(t)=M_{\perp}^{\prime}\left(0_{+}\right) e^{-t / T_{2}} \\
& M_{z^{\prime}}(t)=M_{z}^{0}\left(1-e^{-t / T_{1}}\right)+M_{z^{\prime}}\left(0_{+}\right) e^{-t / T_{1}} .
\end{aligned}
$$

Dabei stehen die Symbole $t_{-}$und $t_{+}($hier $t=0)$ im folgenden jeweils für die Zeitpunkte direkt vor bzw. nach einem RF-Puls. Den durch Gleichung (2.11) beschriebenen Zustand des Systems bezeichnet man als freie Präzession, das mit $T_{2}$ abklingende Signal als freien Induktionsabfall, FID (Free Induction Decay). 


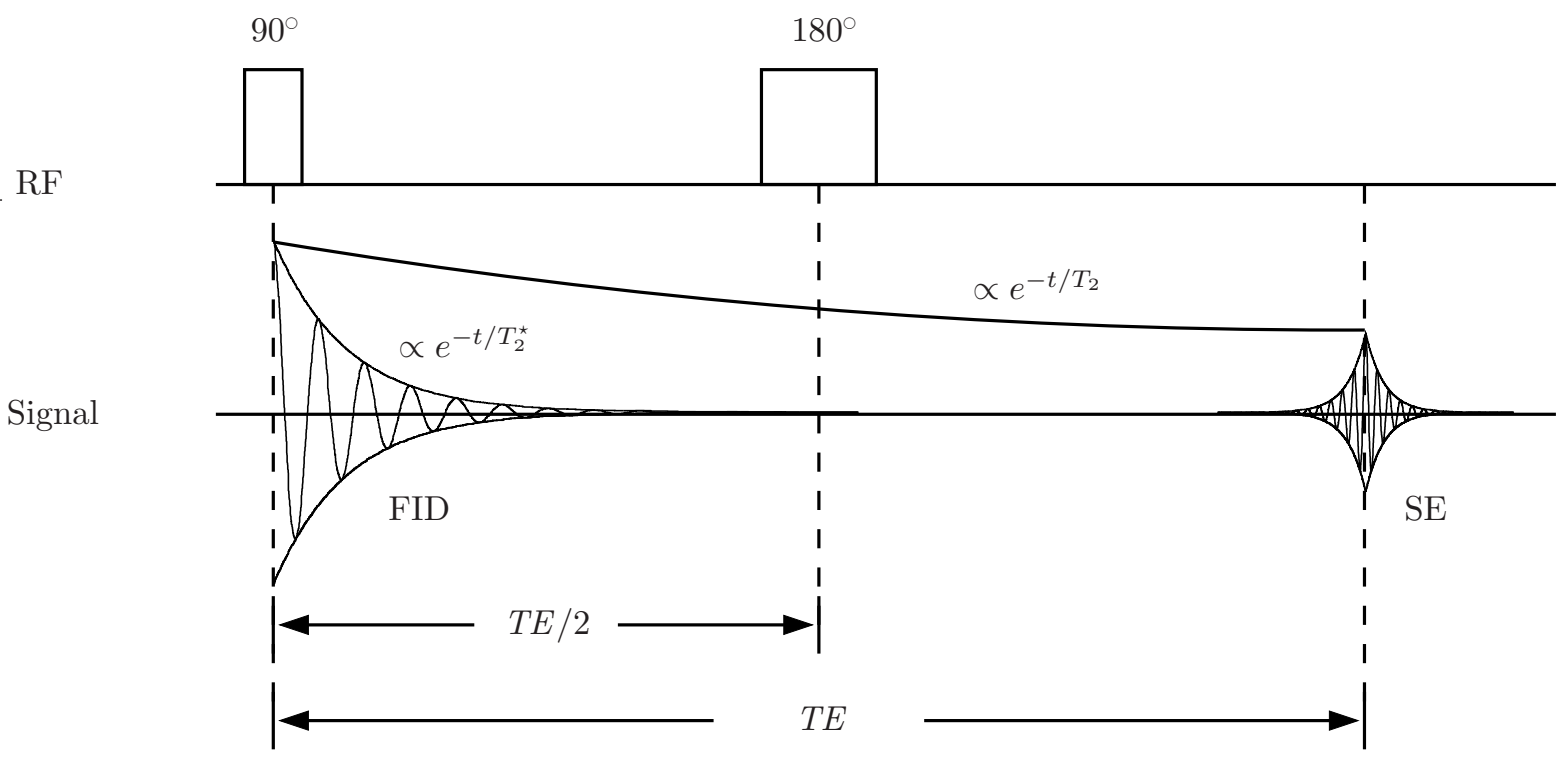

Abbildung 2.2: Einfaches Spin-Echo (SE). Das einfache Spin- oder Hahn-Echo entsteht aufgrund der phaseninvertierenden Wirkung eines der ersten Anregung folgenden zweiten RFPulses, dessen Schaltzeitpunkt TE/2 die Echozeit $T E$ bestimmt. In den Intervallen vor und nach dem refokussierenden Puls muß das System im zeitlichen Mittel die gleichen Larmorfrequenzen aufweisen.

Die Transversalkomponenten der verschiedenen Isochromaten $\boldsymbol{M}(\omega)$, deren für $\omega \neq \omega_{0}$ abweichendes Resonanzverhalten bei der Anregung vernachlässigt wurde, dephasieren aufgrund der unterschiedlichen Präzessionsgeschwindigkeiten im bewegten Bezugssystem mit negativer oder positiver Winkelgeschwindigkeit $\Delta \omega=\omega-\omega_{0}$, je nachdem, ob ihre Frequenz unter- oder oberhalb der anregenden liegt. Nach der Zeit TE/2 hat sich für die Transversalkomponente jeder Spin-Population eine bestimmte Phase $\phi(\Delta \omega)$ in der komplexen Ebene eingestellt. Der darauffolgende $180^{\circ}$-Puls bewirkt einerseits einen für diese Diskussion irrelevanten Vorzeichenwechsel in der $z^{\prime}$-Koordinate und andererseits eine Spiegelung der Magnetisierung an der $\left\{x^{\prime}-z^{\prime}\right\}$-Ebene. Auf diese Weise geht $M_{\perp}^{\prime}$ in das konjugiert komplexe $\left(M_{\perp}^{\prime}\right)^{*}$ über. Die Phasenlage ist damit invertiert, weshalb sich die Transversalkomponenten der einzelnen Isochromaten, die in der weiteren Zeitentwicklung wieder den gleichen Winkel $\phi$ akkumulieren, zum Zeitpunkt TE kohärent aufaddieren und ein sogenanntes einfaches Spin- oder auch Hahn-Echo (SE) der ursprünglichen Systemantwort nach dem ersten Puls bilden. Das erzeugte Signal verhält sich im Prinzip wie der direkt nach der $90^{\circ}$-Anregung beobachtbare freie Induktionsabfall, ist aber im Gegensatz zu ihm zeitlich symmetrisch zur Echo-Zeit TE und in der Amplitude um den Faktor $\exp \left[-T E / T_{2}\right]$ vermindert.

\subsubsection{Stimuliertes Echo}

Neben dem einfachen Spin-Echo (SE) tritt bei der Anwendung von drei oder mehr RFPulsen noch eine zweite ausgezeichnete Klasse von Kohärenzen auf, das stimulierte Echo 


\begin{tabular}{lll}
\hline Typ & Echo Zeit & auslösende+refokussierende Pulse \\
\hline SE & $2 \tau_{1}$ & $(1)+(2)$ \\
SE (2.Ordnung) & $2 \tau_{2}$ & {$[(1)+(2)]+(3)$} \\
STE & $2 \tau_{1}+\tau_{2}$ & $(1)+[(2)+(3)]$ \\
SE & $\tau_{1}+2 \tau_{2}$ & $(2)+(3)$ \\
SE & $2\left(\tau_{1}+\tau_{2}\right)$ & $(1)+(3)$ \\
\hline
\end{tabular}

Tabelle 2.1: Spin-Echo Typen. Kohärenzen in einer 3-Puls-Sequenz

(STE), das für die vorliegende Arbeit von herausragender Bedeutung ist. Dieses Phänomen läßt sich über eine von Woessner [16] eingeführte Darstellung der Auswirkungen eines RF-Pulses zum Zeitpunkt $t$ auf die sich unmittelbar danach einstellende Magnetisierung $\boldsymbol{M}\left(t_{+}\right)$bei gegebener Anfangsbedingung $\boldsymbol{M}(t) \equiv \boldsymbol{M}$ veranschaulichen:

$$
\begin{aligned}
& M_{\perp}^{\prime}\left(t_{+}\right)=M_{\perp}^{\prime} \cos ^{2}\left(\frac{\alpha}{2}\right)-\left(M_{\perp}^{\prime}\right)^{*} \sin ^{2}\left(\frac{\alpha}{2}\right)-M_{z^{\prime}} \sin (\alpha) \\
& M_{z^{\prime}}\left(t_{+}\right)=M_{z^{\prime}} \cos (\alpha)+\frac{1}{2}\left(M_{\perp}^{\prime}+\left(M_{\perp}^{\prime}\right)^{*}\right) \sin (\alpha)
\end{aligned}
$$

Der Term proportional $\mathrm{zu}\left(M_{\perp}^{\prime}\right)^{*}$ in $(2.12 \mathrm{a})$ beinhaltet durch die komplexe Konjugation eine Phasenlageninversion, die nach einem Intervall, das in seiner Dauer der Zeit entspricht, die für die Ausprägung der ursprünglichen Phase vonnöten war, im Zuge der freien Präzession zu einem Echo führt. Eine solche Refokussierung findet statt, wenn eine initiale transversale Magnetisierung vorhanden war, im einfachsten Fall also nach zwei Anregungen. Es handelt sich dann um das bereits diskutierte einfache Spin-Echo. Dagegen ist für das stimulierte Echo der Beitrag $\frac{1}{2}\left(M_{\perp}^{\prime}\right)^{*} \sin (\alpha)$ in der $z^{\prime}$-Koordinate $(2.12 \mathrm{~b})$ der erzeugten Magnetisierung entscheidend, da er nach einem weiteren Puls anteilig Bestandteil der neuen transversalen Komponente wird und, analog argumentiert, ebenfalls ein Echo zur Folge hat. Das ist möglich, weil die Phase in der $z^{\prime}$-Komponente von $\boldsymbol{M}$ konserviert wird. Ein stimuliertes Echo ist deshalb bei einer allgemeinen $\alpha_{1}-\tau_{1}-\alpha_{2}-\tau_{2}-\alpha_{3}-$ Sequenz, also bei der Anwendung der durch die Zeitintervalle $\tau_{i}$ getrennten, zumindest benötigten drei RF-Pulse $\alpha_{i}$, nach der Zeit $2 \tau_{1}+\tau_{2}$ zu erwarten.

Bemerkenswert sind dabei vor allem zwei Dinge. Zum einen ist aus der WoessnerZerlegung ersichtlich, daß das stimulierte Echo durch den Faktor $\frac{1}{2}$ in seiner Intensität mindestens um die Hälfte gegenüber dem ersten FID-Signal vermindert ist. Zusätzlich muß noch der Signalabfall durch Relaxationseffekte berücksichtigt werden. Hier kommt die zweite Eigenart dieses Echo-Typs zum Tragen, denn der für die Erzeugung relevante Magnetisierungsbeitrag ist in der Zeit zwischen dem zweiten und dem dritten Puls nur der $T_{1}$-Equilibrierung ausgesetzt, die deutlich langsamer verläuft als der Zerfall der transversalen Magnetisierung mit $T_{2}$. Dem Nachteil der reduzierten Signalintensität steht also die Möglichkeit gegenüber, das Phasengedächtnis des Spin-Systems über einen längeren Zeitraum erhalten zu können.

Durch die beschriebenen Mechanismen entsteht allgemein bei der Verwendung von $N>2$ RF-Pulsen eine Vielzahl von Echos, insbesondere auch solche höherer Ordnung, 


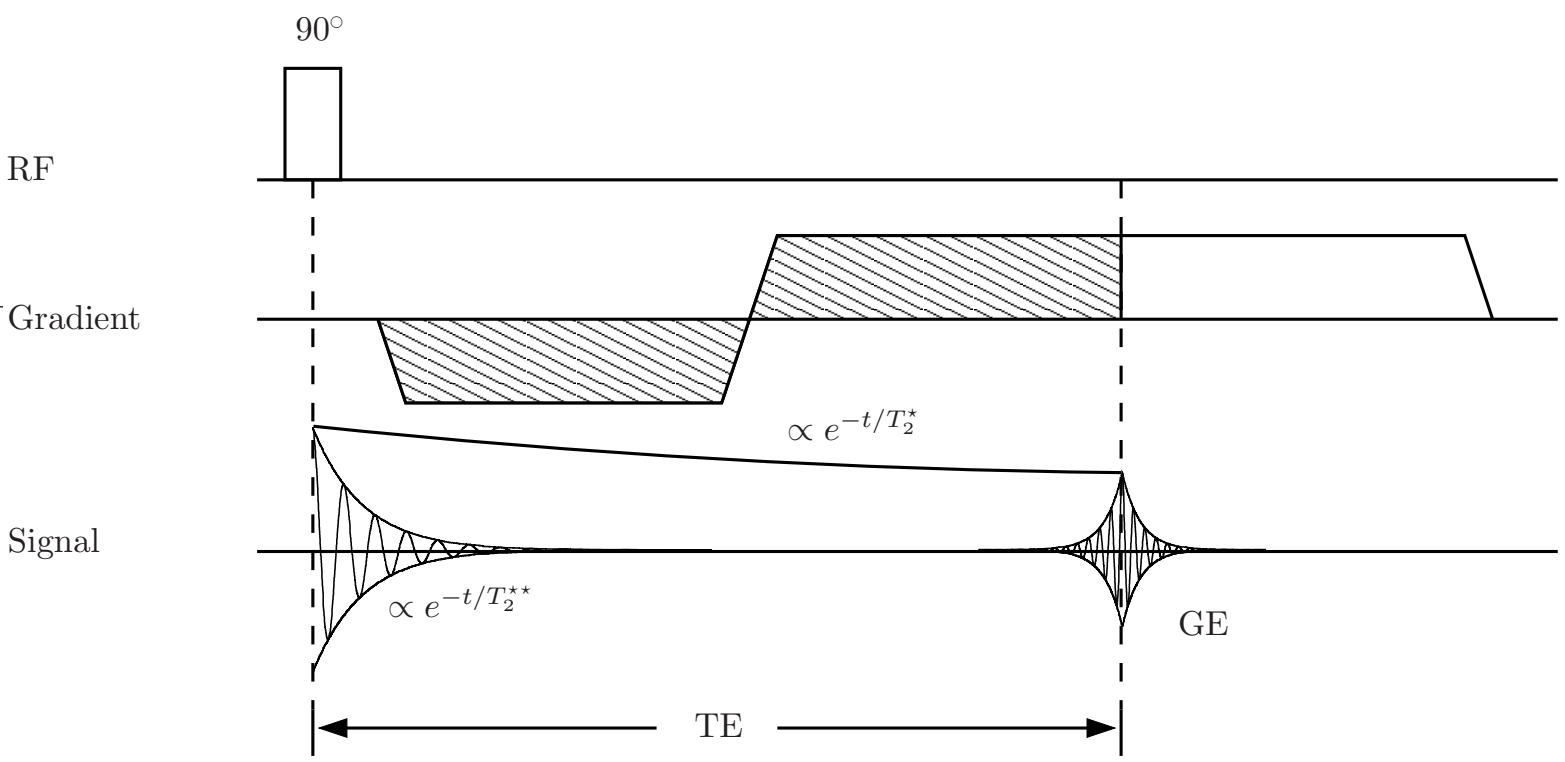

Abbildung 2.3: Gradientenecho (GE). Die durch einen Anregungspuls erzeugte transversale Magnetisierung wird unter der Wirkung eines zeitabhängigen Magnetfeldgradienten über die Probe künstlich de- und rephasiert. Das Integral der Gradientenfunktion verschwindet zum Zeitpunkt TE. In Anwesenheit statischer Magnetfeldgradienten ist die Phasenkohärenz nicht ideal.

d.h. Kohärenzen von Magnetisierungsbeiträgen, die mehrfach rephasiert wurden. Ihr Auftreten und ihre Stärke hängen von der Länge der Zeitintervalle $\tau_{i}$ und der Größe der Flip-Winkel $\alpha_{i}$ ab. In Tabelle 2.1 sind die möglichen Echos einer 3-Puls-Sequenz aufgeführt.

Maximal hat man $\frac{1}{2}\left(\frac{3^{N-1}-1}{2}-N\right)$ Signale unterschiedlicher Intensität zu erwarten [17, S.130]. Mit geschickt plazierten, künstlichen Magnetfeldinhomogenitäten, sogenannte Spoiler und Crusher, lassen sich einige unerwünschte Signale unterdrücken.

\subsubsection{Gradientenecho}

Ein Gradientenfeld $\boldsymbol{B}_{\mathrm{G}}$ im Sprachgebrauch eines NMR-Experimentes moduliert die zKomponente des homogenen Magnetfeldes mit der Stärke $G=\|\boldsymbol{G}\|$ linear entlang einer ausgezeichneten Richtung $\boldsymbol{G} / \boldsymbol{G}$

$$
\boldsymbol{B}_{G}(\boldsymbol{r})=\left(\begin{array}{c}
0 \\
0 \\
\boldsymbol{G} \cdot \boldsymbol{r}
\end{array}\right) \text {. }
$$

Schaltet man solch einen Gradienten nach der Anregung in einem Zeitintervall $\tau$ während der freien Präzessionsphase, so zerfällt die Gesamtmagnetisierung über die Probe rasch aufgrund der dadurch von außen aufgezwungenen räumlichen Variabilität der Larmorfrequenzen $\omega_{0}(\boldsymbol{r})=\omega_{0}(0)+\gamma \boldsymbol{G} \cdot \boldsymbol{r}$. Dies geschieht notwendigerweise schneller als 
beim reinen freien Induktionsabfall mit einer Zeitkonstanten $T_{2}^{\star \star}<T_{2}^{\star}$, die von der Gradientenstärke $G$ abhängt. Am Ort $\boldsymbol{r}$ hat das System zum Zeitpunkt $t$ gegenüber dem Koordinatenursprung die zusätzliche Phase

$$
\phi(\boldsymbol{r}, t)=\gamma \int_{0}^{t} \mathrm{~d} t^{\prime} \boldsymbol{G}\left(t^{\prime}\right) \cdot \boldsymbol{r}
$$

aufgebaut. Durch eine nachfolgende Umkehr der Gradientenrichtung wird der Effekt wieder ins Gegenteil verkehrt und führt nach einem weiteren Zeitintervall der Dauer $\tau$ bei $T E=2 \tau$ zu einem Gradientenecho (GE), das gegenüber der Amplitude des Ausgangssignals um $\exp \left[-T E / T_{2}^{\star}\right]$ abgeschwächt ist. Der Unterschied gegenüber den von Hahn beschriebenen RF-basierten Spin- und stimulierten Echos besteht in der abweichenden Gewichtung des Signals mit der Relaxationskonstanten $T_{2}^{\star}$. Das äußert sich in der NMRBildgebung allgemein in einem anderen Kontrast. Insbesondere wird der Einfluß systemimmanenter Magnetfeldinhomogenitäten, also speziell auch solcher, die aufgrund von Suszeptibilitätsunterschieden entstehen, nicht wie bei RF-induzierten Signalen ausgeglichen, was unter anderem zu Signalauslöschung bzw. -unterdrückung durch das kurze $T_{2}^{\star}$ führt. Weil außerdem die Ortskodierung auf der Erzeugung definierter räumlicher Phasenlagen beruht, treten wegen der verfälschten Larmorfrequenzen namentlich bei Sequenzen, die auf mehrfach refokussierten Gradientenechos aufbauen, in den betroffenen Regionen erhebliche geometrische Verzerrungen auf. Das Gradientenecho hat allerdings den Vorteil, sehr schnelle Bildgebungssequenzen zu ermöglichen.

\subsection{Diffusion und das NMR-Signal}

Schon in seiner grundlegenden Arbeit zum Spin-Echo untersuchte Hahn [6] auch die Auswirkungen von diffusiven Prozessen auf das NMR-Signal. Besonders in den Anfängen der Kernspinresonanz-Methode bei teilweise noch recht unzulänglichen statischen Magnetfeldern erwiesen sich diese Überlegungen als unerläßlich, weil durch Inhomogenitäten in $\boldsymbol{B}_{0}$ den Atomkernen in Flüssigkeiten als Konsequenz ihrer thermischen (Brownschen) Bewegung stochastisch zu behandelnde, zeitabhängige Larmorfrequenzen aufgeprägt werden. Folglich hat eine Gruppe von mobilen Spins mit einer zum Zeitpunkt der ersten Anregung gleichen Frequenz im späteren Verlauf aufgrund von (Selbst-) Diffusion eine breite Phasenverteilung. Deswegen werden nach einem zweiten Puls nur die Spins stationärer Kerne vollständig kohärent zu einem Echo refokussiert, so daß man eine Reduktion der Signalamplitude beobachtet. Weil sie den Signalabfall bei der Bestimmung der Relaxationszeiten verfälscht, ist Diffusion in diesem Sinne eigentlich ein Schmutzeffekt. Ihr Einfluß bietet jedoch andererseits die Möglichkeit, durch eine makroskopische Messung molekulare Dynamik zu untersuchen. Einen guten Einblick in diesen Themenbereich gewinnt man durch die Lektüre der Übersichtsartikel von Price [18, 19]. 


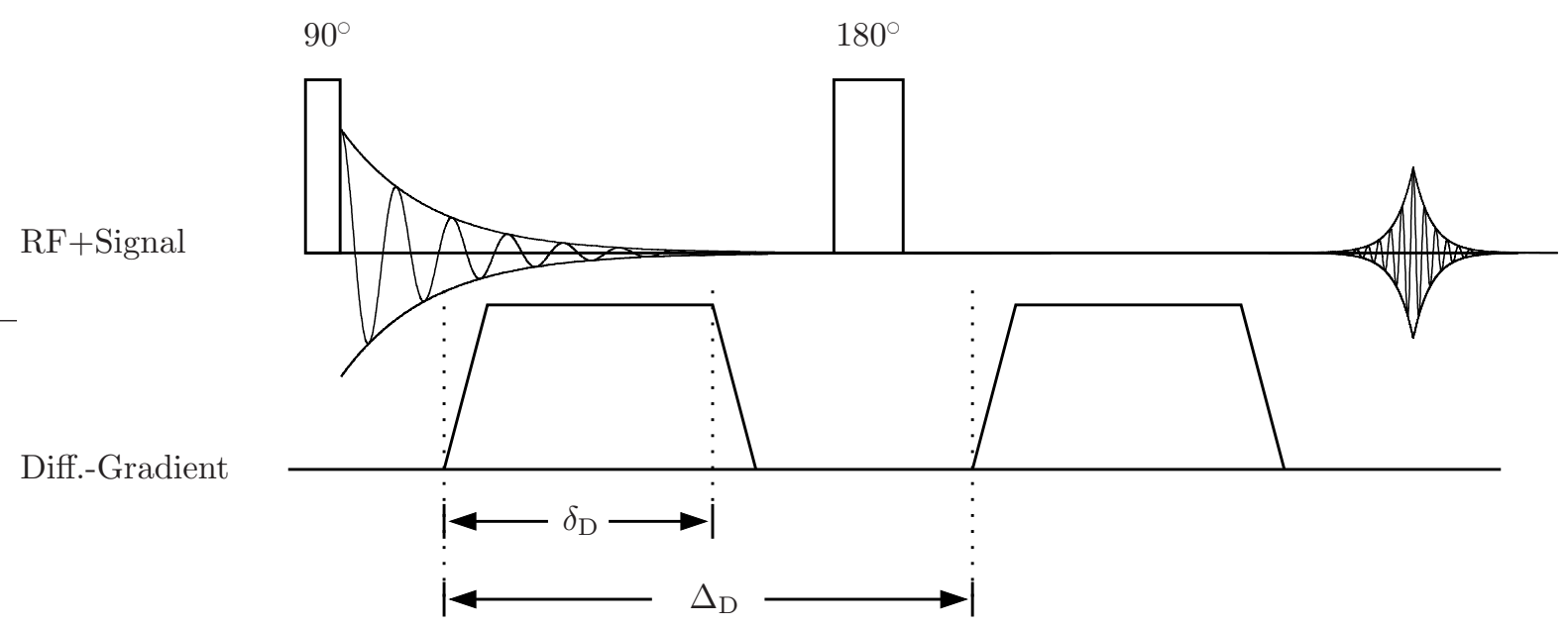

Abbildung 2.4: Stejskal-Tanner-Sequenz. Diffusionssensibilisierung mit gepulsten Magnetfeldgradienten.

\subsubsection{Die Stejskal-Tanner-Sequenz}

Zur Bestimmung von Diffusionskonstanten mittels Kernspinresonanz werden dedizierte, aus Gleichung (2.13) bekannte räumlich variable Magnetfelder eingesetzt. Weil ein während des gesamten Versuches zeitlich konstanter Gradient ungünstige Auswirkungen auf die benötigte Stärke der RF-Anregungen und die Breite der Echos hat, benutzt man eine auf Stejskal und Tanner [13] zurückgehende gepulste Schaltung, um die Sequenz für die Brownsche Bewegung zu sensibilisieren. Mit dieser Methode lassen sich deshalb auch sehr kleine Beweglichkeiten messen; außerdem ist die Diffusionszeit prinzipiell sinnvoller definiert.

In der Stejskal-Tanner-Sequenz, auch als PGSE (Pulsed-Gradient Spin-Echo)-Sequenz bekannt, ordnet man, wie in Abbildung 2.4 dargestellt, nach einer $90^{\circ}$-Anregung zwei Gradienten $\boldsymbol{G}$ gleicher Amplitude und der Puls-Dauer $\delta_{\mathrm{D}}$ im relativen Startabstand $\Delta_{\mathrm{D}}$ als Diffusionskodierer an den Seiten eines $180^{\circ}$-Refokussierers an. Es handelt sich also um eine geringfügige, aber folgenreiche Variation der Hahn-Echo Pulsfolge. Die Kernspins werden mit Hilfe des ersten, zum Zeitpunkt $t_{1}$ geschalteten Gradienten räumlich über ihre Larmorfrequenz markiert (,Spin-Labelling“) und durch den zweiten Gradienten über die translationsbedingte, für jedes Teilchen individuelle Phasenverschiebung mit ihrem Anfangszustand verglichen. Zum Zeitpunkt TE hat ein einzelner Spin mit der Trajektorie $\boldsymbol{r}(t)$ also die relative Phase

$$
\phi=\gamma\left[\int_{t_{1}}^{t_{1}+\delta_{\mathrm{D}}} \mathrm{d} t \boldsymbol{G}(t) \cdot \boldsymbol{r}(t)-\int_{t_{1}+\Delta_{\mathrm{D}}}^{t_{1}+\Delta_{\mathrm{D}}+\delta_{\mathrm{D}}} \mathrm{d} t \boldsymbol{G}(t) \cdot \boldsymbol{r}(t)\right]
$$

akkumuliert. Der abschwächende Effekt auf die Amplitude des gemessenen Signals $S_{G}(t)$ wächst mit der Beweglichkeit der Teilchen und wird durch die Phasenverteilung $\mathcal{P}_{G}(\phi, t)$ 
(a)

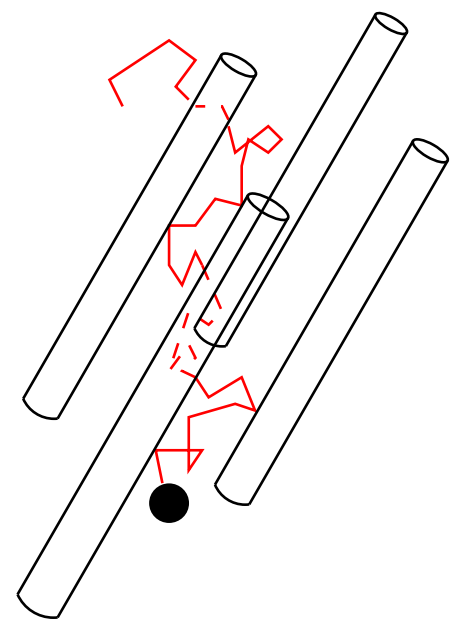

(b)

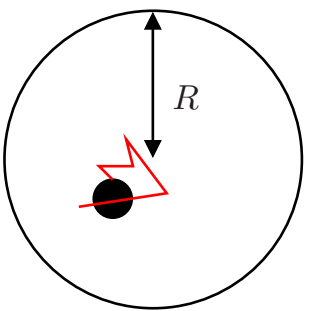

$D \Delta_{\mathrm{D}} / R^{2} \gg 1$
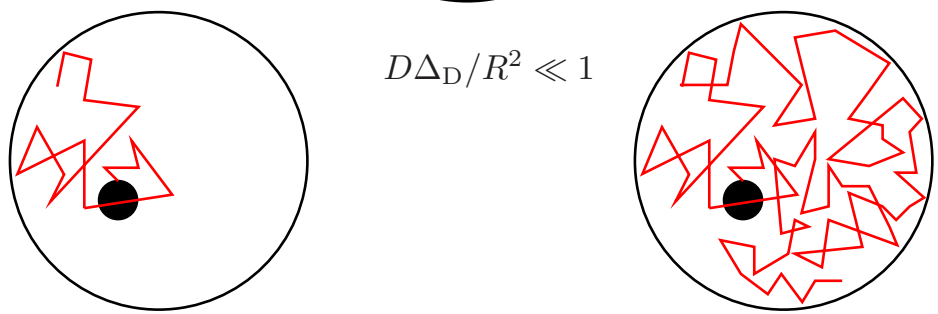

Abbildung 2.5: Einfluß der Geometrie auf Diffusionsmessungen. a) obstruierte Diffusion b) beschränkte Diffusion: Abhängigkeit der gemessenen Beweglichkeit von der Zeitskala.

des Spin-Systems bestimmt

$$
S_{\boldsymbol{G}}(T E)=S_{\mathbf{0}}(0) e^{-T E / T_{2}} \int_{-\infty}^{\infty} \mathrm{d} \phi \mathcal{P}_{\boldsymbol{G}}(\phi, T E) e^{\mathrm{i} \phi} .
$$

Dabei ist $\mathcal{P}_{\boldsymbol{G}}(\phi, t)$ einerseits durch die Gradientenschaltung $\boldsymbol{G}(t)$, also den Parametersatz $\left\{\delta_{\mathrm{D}}, \Delta_{\mathrm{D}}, \boldsymbol{G}\right\}$, und andererseits durch die molekulardynamischen Eigenschaften des Systems determiniert. Der Einfluß der Diffusion kann deswegen durch den relativen Signalabfall

$$
A\{\boldsymbol{G}(t)\}:=\frac{S_{\boldsymbol{G}}(T E)}{S_{\mathbf{0}}(T E)}
$$

beschrieben und von der $T_{2}$-Relaxation separiert werden.

\subsubsection{Diffusion in biologischen Strukturen}

Im allgemeinen reicht die Modellierung thermischer Bewegung durch eine einfache Diffusionskonstante $D$ nicht aus. Insbesondere muß man sich häufig von der Ortsunabhängigkeit und der skalaren Beschreibung verabschieden. Definiert man $D$ außerdem in einem homogenen und isotropen, aber räumlich beschränkten Medium maximaler Ausdehnung $R$ über das Ensemble-gemittelte Verschiebungsquadrat eines frei in einem dreidimensionalen Raum diffundierenden Teilchens

$$
\left\langle(\Delta \boldsymbol{r})^{2}\right\rangle=6 D \Delta_{\mathrm{D}}
$$

so wird man zwar für hinreichend kleine $\Delta_{\mathrm{D}}$, d. h. $\xi:=D \Delta_{\mathrm{D}} / R^{2} \ll 1$, annähernd ein der freien Bewegung entsprechendes $D$ messen, sobald aber die Moleküle während der 
Diffusionszeit mit den Wänden in Wechselwirkung treten $(\xi \approx 1)$, verringert sich der so bestimmte Wert wegen der geometrischen Randbedingung $\|\Delta \boldsymbol{r}\| \leq R$. Auf diese Weise wird $D$ sogar zu einer zeitabhängigen Funktion.

In biologischem Gewebe ist die Bewegung von Wassermolekülen einer komplizierten Dynamik unterworfen. Neben beschränkter Diffusion, also der Einschränkung der Dynamik auf einen endlichen Raum während des Experiments, kann die freie Ausbreitung der Teilchen in eine bestimmte Richtung auch nur durch kompakte Zellstrukturen, semipermeable Membrane und ähnlichen Hindernisse be- aber nicht verhindert sein, was hier als obstruierte Diffusion bezeichnet werden soll (siehe Abbildung 2.5). Außerdem tragen aktive Transportprozesse durch Membrane sowie chemische Interaktionen und Austauschreaktionen der Wasserstoffatome mit Makromolekülen zur Verkomplizierung des Sachverhalts bei.

Die Grundannahme, daß sich mikroskopisch inhomogenes Gewebe auf entsprechend grob gewählten räumlichen und zeitlichen Skalen lokal wieder als homogenes, aber im allgemeinen anisotropes Medium auffassen läßt, führt auf die Diffusionstensorbildgebung. Ein Diffusionstensor zweiter Ordnung D kann als einfachstes Modell für die Dynamik der Dichte $\rho(\boldsymbol{r}, t)$ eines Stoffes in einer Umgebung betrachtet werden, in welcher der Vektor der Teilchenstromdichte $\boldsymbol{j}$ nicht mehr mit der Richtung des Konzentrationsgradienten übereinstimmt. Das modifizierte erste Ficksche Gesetz lautet dann

$$
\boldsymbol{j}(\boldsymbol{r}, t)=-\mathbf{D} \nabla \rho(\boldsymbol{r}, t) .
$$

Die Übertragung dieser Beschreibung auf Selbstdiffusionsprozesse in biologischem Gewebe und das Verfahren zur quantitativen Bestimmung von D gehen auf Basser et al. [14, 15] zurück, nachdem in Vorarbeiten (siehe Referenzen in [14]) gezeigt worden war, daß der skalare Diffusionskoeffizient in gerichtetem Gewebe wie der weißen Hirnsubstanz oder Muskeln eine Abhängigkeit von der Lage der kodierenden Gradientenpulse relativ zur makroskopischen Faserrichtung mit einem Maximum bei paralleler Orientierung besitzt. Ein herausragendes Ergebnis des neuen Verfahrens war die Tatsache, daß diese Hauptfaserrichtung erfolgreich durch den zum größten Eigenwert korrespondierenden Eigenvektor des Diffusionstensors beschrieben und deshalb aus einer drastisch reduzierten Anzahl von Messungen bestimmt werden kann (siehe Kapitel 3). Weil es sich bei dem gemessenen D nicht um eine skalenunabhängige Größe handelt, und die Einträge der entsprechenden Matrix von den experimentellen Parametern abhängen, spricht man auch von einem scheinbaren oder effektiven Diffusionstensor $\mathbf{D}_{\text {eff }}$ und quasifreier Diffusion.

In dieser Arbeit liegt das Hauptaugenmerk auf der Protonenbewegung im ZNS (Zentrales Nervensystem), inbesondere in der weißen Hirnsubstanz. Dieses Gewebe besteht aus sich zu Bündeln zusammenschließenden langreichweitigen Axonen. Das sind Auswüchse von Neuronen, deren Zellkörper und Dendriten selbst einen Großteil der grauen Substanz ausmachen. Die Axone der weißen Hirnsubstanz sind gewissermaßen Datenautobahnen und verbinden langreichweitig funktionelle Zentren. Sie werden in vielen Bereichen beim erwachsenen, gesunden Menschen von Gliazellen (Oligodendrozyten) ummantelt, wodurch sich die elektrische Leitfähigkeit verbessert. Die Myelinschicht stellt dabei eine zusätzliche Bewegungsbarriere für Wassermoleküle dar. Aus Studien an Neugeborenen, 
bei denen diese Lipidhülle noch nicht voll ausgeprägt ist, weiß man, daß sie die Richtungsabhängigkeit der Diffusion zwar wesentlich beeinflußt, aber nicht allein bestimmt. Axone haben einen Durchmesser von der Größenordnung $10 \mu \mathrm{m}$, der aber genauso wie die Packungsdichte einer gewissen Schwankungsbreite unterliegt. Setzt man den Selbstdiffusionskoeffizienten von Wassermolekülen entlang der Hauptdiffusionsrichtung der weißen Substanz voraus, der sich in einem Bereich von ca. $0,5-2,0 \cdot 10^{-9} \mathrm{~m}^{2} / \mathrm{s}$ bewegt und typischerweise bei $1,0 \cdot 10^{-9} \mathrm{~m}^{2} / \mathrm{s}$ liegt, so tritt ein Teilchen innerhalb eines Axons nach einem charakteristischen Zeitintervall von $4 \mathrm{~ms}$ in Wechselwirkung mit den Zellwänden.

\subsubsection{Quantitative Beschreibung mit der Bloch-Torrey-Gleichung}

Die Auswirkungen thermischer Bewegung auf die gemessene Kernspin-Relaxation werden einer quantitativen Behandlung durch die Erweiterung der Blochschen Formulierung um einen lokalen Diffusionsterm nach Torrey [20] zugänglich

$$
\frac{\partial \boldsymbol{M}(\boldsymbol{r}, t)}{\partial t}=\gamma \boldsymbol{M} \times \boldsymbol{B}(\boldsymbol{r}, t)-\frac{M_{x} \boldsymbol{e}_{x}+M_{y} \boldsymbol{e}_{y}}{T_{2}}-\frac{\left(M_{z}-M_{z}^{0}\right)}{T_{1}} \boldsymbol{e}_{z}+\nabla \cdot(\mathbf{D} \nabla \boldsymbol{M}),
$$

wobei die Verwendung eines Diffusionstensors anstelle eines Skalars auf Stejskal [21] zurückgeht, der damit bereits anisotrope, obstruierte Diffusion zu beschreiben versuchte. Driftterme, die aufgrund der Kraftwirkung von Magnetfeldgradienten auf die Atomkerne zur Teilchenstromdichte beitragen könnten, treten zwar in der allgemeinen Formulierung der Bloch-Torrey-Gleichung (2.20) auf, werden hier aber nicht berücksichtigt. Beachtenswerterweise hat Gleichung (2.7) durch den Diffusionsterm ihre Ortsunabhängigkeit eingebüßt. Die Magnetisierung $\boldsymbol{M}$ bezieht sich jetzt also auf ein Volumenelement am Ort $\boldsymbol{r}$.

Für die Berechnung des Effekts der Diffusionskodierung in der Stejskal-Tanner-Sequenz in einem homogenen, anisotropen und unendlich ausgedehnten Medium betrachtet man die komplexe Transversalmagnetisierung $M_{\perp}$ im Laborsystem (siehe z. B. [22, Kap.1]), mit $\boldsymbol{B}(\boldsymbol{r}, t)=\left(0,0, B_{0}+\boldsymbol{G}(t) \cdot \boldsymbol{r}\right)^{\mathrm{T}}$

$$
\frac{\partial M_{\perp}(\boldsymbol{r}, t)}{\partial t}=\left(-\mathrm{i} \omega_{0}-\frac{1}{T_{2}}-\mathrm{i} \gamma \boldsymbol{r} \cdot \boldsymbol{G}(t)\right) M_{\perp}+\nabla \cdot\left(\mathbf{D} \nabla M_{\perp}\right) .
$$

$\mathrm{Zu}$ bemerken ist, daß sich die Phaseninversion durch den $180^{\circ}$-Pulse mathematisch in einen Vorzeichenwechsel von $\boldsymbol{G}$ übersetzt. Der Ansatz

$$
M_{\perp}(\boldsymbol{r}, t)=: m(t) \exp [-\mathrm{i} \boldsymbol{k}(t) \cdot \boldsymbol{r}] \exp \left[-\left(\mathrm{i} \omega_{0}+\frac{1}{T_{2}}\right) t\right]
$$

mit

$$
\boldsymbol{k}(t):=\gamma \int_{0}^{t} \mathrm{~d} t^{\prime} \boldsymbol{G}\left(t^{\prime}\right)
$$

führt auf eine gewöhnliche Differentialgleichung für die Größe $m(t)$, deren zeitliche Än- 
derung auf die Diffusion zurückzuführen ist

$$
\frac{\mathrm{d} m(t)}{\mathrm{d} t}=-m(t) \boldsymbol{k}(t)^{T} \mathbf{D} \boldsymbol{k}(t)
$$

Die Lösung, ausgedrückt durch die Transversalmagnetisierung $M_{\perp}\left(0_{+}\right)$unmittelbar nach Applikation des ersten $90^{\circ}$-Pulses, lautet

$$
\begin{aligned}
m(t) & =M_{\perp}\left(0_{+}\right) \exp \left[-\int_{0}^{t} \mathrm{~d} t^{\prime} \boldsymbol{k}\left(t^{\prime}\right)^{\mathrm{T}} \mathbf{D} \boldsymbol{k}\left(t^{\prime}\right)\right] \\
& =M_{\perp}\left(0_{+}\right) \exp [-\operatorname{tr}(\mathbf{b} \mathbf{D})] .
\end{aligned}
$$

Man hat also die Behandlung des diffusiven Signalabfalls auf die Berechnung der Spur des Matrixproduktes des Diffusionstensors mit der sogenannten b-Matrix

$$
\mathbf{b}(t):=4 \pi^{2} \int_{0}^{t} \mathrm{~d} t^{\prime} \boldsymbol{k}\left(t^{\prime}\right) \boldsymbol{k}\left(t^{\prime}\right)^{\mathrm{T}}
$$

reduziert, die über das Integral des dyadischen Produktes der $\boldsymbol{k}$-Vektoren gegeben ist. Wenn D über das Meßintervall nicht konstant sein sollte, dient Gleichung (2.25b) gleichzeitig als implizite Definition eines zeitgemittelten Effektivwertes. Weil die Struktur der b-Matrix durch die Richtung des Diffusionsgradienten bereits bekannt ist, muß für eine spezifische Sequenz nur noch der Faktor

$$
b(t):=\operatorname{tr}(\mathbf{b}(t))=\gamma^{2} \int_{0}^{t} \mathrm{~d} t^{\prime} G\left(t^{\prime}\right)^{2},
$$

der als b-Wert des Experiments bezeichnet wird, in Abhängigkeit von der Gradientenschaltung berechnet werden. Im gewöhnlichen Sprachgebrauch benutzt man nur die Größe $b \equiv b(T E)$. Für ein homogenes isotropes Medium ergibt sich für eine Sequenz mit Trapez-Gradienten zum Beispiel zur Echozeit TE der diffusionsbedingte Abfall

$$
A\{\boldsymbol{G}(t)\}=\exp [-b D]
$$

$\operatorname{mit}[15]$

$$
b=(\gamma G)^{2}\left[\delta_{\mathrm{D}}^{2}\left(\Delta_{\mathrm{D}}-\frac{\delta_{\mathrm{D}}}{3}\right)+\frac{\epsilon^{3}}{30}-\frac{\delta_{\mathrm{D}} \epsilon^{2}}{6}\right] .
$$

Das Hochfahren der Gradienten auf die Stärke $G$ in der endlichen Zeit $\epsilon$ (Ramp-Zeit) wurde dabei als zeitlich linearer Vorgang angenommen. Im Grenzfall $\delta_{\mathrm{D}} \rightarrow 0$ entfällt der Term $\delta_{\mathrm{D}} / 3$ in (2.29). Für endliche Schaltzeiten bezeichnet man $\Delta_{\mathrm{D}}-\delta_{\mathrm{D}} / 3$ als effektive Diffusionszeit. 


\subsubsection{Auflösung komplexer Geometrien}

Der Diffusionstensor bildet in komplexen Geometrien vielfach die zugrundeliegenden Strukturen nur unzulänglich ab. Beispielsweise lassen sich damit beliebig kreuzende Nervenfaserbündel in einem entsprechenden Volumenelement der weißen Hirnsubstanz nicht vollständig charakterisieren, denn die drei ausgezeichneten Richtungen des Tensors liegen orthogonal zueinander. Somit verbietet sich in diesem Fall eine einfache Bestimmung der Faserrichtungen anhand der Eigenstruktur von D, die für Gewebe mit homogener Textur und einer einzigen ausgezeichneten Orientierung so erfolgreich ist. Darüberhinaus sieht man in Experimenten mit hohen b-Werten, daß der Diffusionseffekt sich nicht mehr in einem monoexponentiellen Zerfall der Magnetisierung äußert, sondern durch mehr als eine Zeitskala bestimmt zu werden scheint. (Anbei bemerkt sind wohl einige der in der Literatur dokumentierten Beobachtungen aber auch auf Verfälschungen durch den Rauschuntergrund (siehe Abschnitt 3.3.2) zurückzuführen.) Versuche, die makroskopische Theorie empirisch durch Verwendung von mehreren Diffusionstensoren für verschiedene mikroskopische Kompartimente und einem multiexponentiellen Modell zu retten, ergaben inkonsistente Schätzwerte für die Größe der beitragenden Volumenanteile wie zum Beispiel intra- und extrazelluläres Wasser. Außerdem stellt sich das Problem, eine große Anzahl von freien Parametern numerisch stabil zu schätzen. Der Ansatz vermittelt allerdings einen intuitiven Zugang zur Erklärung der Zulässigkeit und Aussagekraft des einfachen Diffusionstensormodells für kleine b-Werte. Gegeben seien $N$ nicht wechselwirkende Kompartimente, charakterisiert durch $\mathbf{D}_{i}, i=1, \ldots N$, und die Volumenanteile $f_{i}$. Eine Taylor-Entwicklung um $\operatorname{tr}\left(\mathbf{b D}_{i}\right)=0$ des Diffusionsabfalls ergibt [23]

$$
\begin{aligned}
A & =\sum_{i=1}^{N} f_{i} \exp \left[-\operatorname{tr}\left(\mathbf{b} \mathbf{D}_{i}\right)\right] \\
& \left.\approx \sum_{i=1}^{N} f_{i}\left[1-\operatorname{tr}\left(\mathbf{b} \mathbf{D}_{i}\right)+\frac{1}{2} \operatorname{tr}\left(\mathbf{b} \mathbf{D}_{i}\right)\right)^{2}+\ldots\right] \\
& \approx \exp \left[-\operatorname{tr}\left(\mathbf{b} \mathbf{D}_{\text {eff }}\right)\right]
\end{aligned}
$$

mit

$$
\sum_{i=1}^{N} f_{i}=1 \quad \text { und } \quad \mathbf{D}_{\text {eff }}=\sum_{i=1}^{N} f_{i} \mathbf{D}_{i}
$$

Demnach ist der Signalabfall bei geeigneter Parameterwahl nur durch einen einzigen, effektiven Diffusionstensor $\mathbf{D}_{\text {eff }}$ charakterisiert.

Die tiefere Ursache für das Versagen von Diffusionstensormodellen bei hohen b-Werten liegt in der durch die Voraussetzung quasifreier Diffusion implizierten Annahme einer Gaußschen Form der Verschiebungswahrscheinlichkeit eines Teilchens begründet. Obwohl sie nicht im Rahmen dieser Arbeit genauer berücksichtigt werden kann, sei deshalb an dieser Stelle noch eine Vorgehensweise [24, 25, 26] erwähnt, mit der man versucht, auch komplexe Geometrien mit diffusionsgewichteter NMR aufzulösen. Sie basiert auf einem 
Spezialfall des Stejskal-Tanner Experimentes, in dem man die Zeit $\delta_{\mathrm{D}}$ so klein wählt, daß während der Applikation der Gradientenpulse praktisch keine Translation eines Teilchens durch Diffusion stattfindet, bzw. in abgemilderter und experimentell realisierbarer Form, daß der Hauptanteil der diffusionsbedingten Dephasierung näherungsweise auf die Bewegung zwischen den Gradientenpulsen zurückzuführen ist, d. h.

$$
\delta_{\mathrm{D}} \ll \Delta_{\mathrm{D}}
$$

Die Voraussetzung (2.31) wird in der englischen Literatur als „SGP-condition“ (Short Gradient Pulse) bezeichnet. Mit dieser Approximation läßt sich der Signalabfall durch die mittlere Teilchendichte $\rho(\boldsymbol{r})$ und die bedingte Wahrscheinlichkeit $\mathcal{P}\left(\boldsymbol{r} \mid \boldsymbol{r}_{0}, \Delta_{\mathrm{D}}\right)$ ausdrücken, einen Atomkern, der sich zunächst am Ort $\boldsymbol{r}_{0}$ befindet, nach der Zeit $\Delta_{\mathrm{D}}$ am Ort $\boldsymbol{r}$ zu finden

$$
A\left(\boldsymbol{G}, \Delta_{\mathrm{D}}, \delta_{\mathrm{D}}\right)=\int \mathrm{d} \boldsymbol{r}_{0} \rho\left(\boldsymbol{r}_{0}\right) \int \mathrm{d} \boldsymbol{r} \mathcal{P}\left(\boldsymbol{r} \mid \boldsymbol{r}_{0}, \Delta_{\mathrm{D}}\right) \exp \left[\mathrm{i} \gamma \delta_{\mathrm{D}} \boldsymbol{G} \cdot\left(\boldsymbol{r}-\boldsymbol{r}_{0}\right)\right] .
$$

Die Funktion $\mathcal{P}\left(\boldsymbol{r} \mid \boldsymbol{r}_{0}, \Delta_{\mathrm{D}}\right)$ nennt man auch den Propagator. Führt man formal die Integration über $\boldsymbol{r}_{0}$ aus, offenbart sich für $A$ bei festen Parametersatz $\left\{\delta_{\mathrm{D}}, \Delta_{\mathrm{D}}\right\}$ eine Fourier-Beziehung

$$
A(\boldsymbol{q})=\int \mathrm{d} \boldsymbol{R} \overline{\mathcal{P}}\left(\boldsymbol{R}, \Delta_{\mathrm{D}}\right) \exp [\mathrm{i} 2 \pi \boldsymbol{q} \cdot \boldsymbol{R}]
$$

zu einem gemittelten Propagator

$$
\overline{\mathcal{P}}\left(\boldsymbol{R}, \Delta_{\mathrm{D}}\right):=\int \mathrm{d} \boldsymbol{r}_{0} \rho\left(\boldsymbol{r}_{0}\right) \mathcal{P}\left(\boldsymbol{r}_{0}+\boldsymbol{R} \mid \boldsymbol{r}_{0}, \Delta_{\mathrm{D}}\right)
$$

in Abhängigkeit von dem zur Verschiebung $\boldsymbol{R}$ konjugierten Wellenvektor

$$
\boldsymbol{q}:=\frac{1}{2 \pi} \gamma \boldsymbol{G} \delta_{\mathrm{D}}
$$

Im Fall freier Diffusion ist die Verschiebungswahrscheinlichkeit ortsunabhängig und läßt sich wegen $\mathcal{P}\left(\boldsymbol{R}, \Delta_{\mathrm{D}}\right)=\overline{\mathcal{P}}\left(\boldsymbol{R}, \Delta_{\mathrm{D}}\right)$ direkt über die Invertierung von Gleichung (2.33) bestimmen. Eine fundamentale Einsicht gewährt die Betrachtung des Grenzfalls langer Diffusionszeiten $\Delta_{\mathrm{D}} \rightarrow \infty$ in beschränkten Geometrien, für die die Spin-Trajektorien als unabhängig von ihrer Startposition angesehen werden können

$$
\mathcal{P}\left(\boldsymbol{r} \mid \boldsymbol{r}_{0}, \Delta_{\mathrm{D}}\right)=\rho(\boldsymbol{r}) .
$$

Dann stellt sich der mittlere Propagator als Autokorrelation

$$
\overline{\mathcal{P}}\left(\boldsymbol{R}, \Delta_{\mathrm{D}}\right)=\int \mathrm{d} \boldsymbol{r} \rho\left(\boldsymbol{r}_{0}\right) \rho\left(\boldsymbol{r}_{0}+\boldsymbol{R}\right)
$$

der $\rho(\boldsymbol{r})$ entsprechenden Trägerfunktion des Systems dar. Der zugehörige Diffusionsabfall 
erhält, wenn man das Wiener-Chintschin-Theorem bemüht, dadurch die Gestalt einer inkohärenten Streufunktion

$$
A(\boldsymbol{q})=\left|F(\boldsymbol{q})^{2}\right|
$$

als Betragsquadrat des Formfaktors

$$
F(\boldsymbol{q}):=\int \mathrm{d} \boldsymbol{r} \rho(\boldsymbol{r}) e^{-\mathrm{i} 2 \pi \boldsymbol{q} \cdot \boldsymbol{r}} .
$$

Man gewinnt also indirekt durch Interferenzphänomene Informationen über die Form und Größe des Potentials, in dem sich die Brownschen Teilchen bewegen.

Der große Vorteil von Gleichung (2.32), die die Grundlage aller „,-space“-Ansätze bildet, besteht darin, daß sie einen zunächst modellfreien Zugang zur Abhängigkeit einer makroskopischen Meßgröße von der mikroskopischen Dynamik liefert. Das (effektive) Diffusionstensormodell ist darin als Grenzfall für kleine Wellenlängen $q$ enthalten [27]. In der medizinischen Bildgebung lassen sich die SGP-Bedingung und die erforderlichen hohen $q$-Werte aus physiologischen und technischen Gründen nicht angemessen realisieren. Die Daten entziehen sich also einer direkten, physikalisch korrekten Interpretation mit Hilfe des mittleren Propagators. Es gibt aber vielversprechende Arbeiten [28, 29], die auf der $q$-Raum-Methode aufbauend versuchen, die Beschränkungen der Diffusionstensorbildgebung zu überwinden.

\subsection{NMR-Bildgebung}

Um dem NMR-Signal ortsaufgelöste Bilder zu entlocken, verfolgt man im Grunde zwei komplementäre Strategien, die unter dem Begriff MRI (Magnetic Resonance Imaging) zusammengefaßt werden: die räumliche Kodierung der ausgelenkten Magnetisierung über die Larmor-Frequenz und die selektive Anregung von Schichten. Beide Ansätze nutzen dabei aus, daß sich einer Probe durch Magnetfeldgradienten definierte Frequenzvariationen aufprägen lassen, und man auf diese Weise Einfluß auf das Präzessions- und Resonanzverhalten der Spins erhält. Durch Anwendung der Stejskal-Tanner-Schaltung vor einer bildgebenden Sequenz ist es außerdem möglich, eine lokal aufgelöste Messung der Diffusionseigenschaften in einem Versuchsobjekt vorzunehmen. Wird dabei der vollständige Diffusionstensor bestimmt, spricht man von DTI (Diffusion Tensor Imaging).

\subsubsection{Schichtselektive Pulse}

Ein in Richtung der Schichtnormalen geschalteter Magnetfeldgradient $\boldsymbol{G}_{\mathrm{s}}$ bewirkt eine ortsabhängige Änderung der Larmor-Frequenzen

$$
\omega(\boldsymbol{r})=\omega_{0}+\gamma \boldsymbol{G}_{\mathrm{s}} \cdot \boldsymbol{r},
$$


so daß die Kernspins in den dazu orthogonalen Ebenen jeweils taktgleich präzedieren. Appliziert man gleichzeitig einen Anregungspuls

$$
B_{1}(t)=B_{1}^{\mathrm{e}}(t) e^{-\mathrm{i} \omega_{1} t}
$$

mit der Trägerfrequenz $\omega_{1}$, erfahren nur die Atome einen relevanten Energieübertrag, die sich in dem durch die Frequenzbandbreite $\Delta \omega_{1}$ der Einhüllenden $B_{1}^{\mathrm{e}}(t)$ implizit definierten Ortsraum

$$
\left|\omega_{1}-\omega(\boldsymbol{r})\right| \leq \frac{\Delta \omega_{1}}{2}
$$

befinden. Durch Variation von $\omega_{1}$ läßt sich also der Aufpunktvektor $\boldsymbol{r}_{0}$ der Mittenebene dieser Schicht verschieben, und über die Gradientenstärke $G_{s}$ oder die Bandbreite ihre Dicke einstellen.

Zur Bestimmung des genauen Anregungsprofils muß man die instantane Bloch-Gleichung (2.10) in Anwesenheit eines zeitlich konstanten Gradientenfeldes lösen. Für kleine Flipwinkel entkoppeln die Gleichungen für Longitudinal- und Transversalmagnetisierung, $M_{z}^{\prime}$ und $M_{\perp}^{\prime}$, so daß das Magnetisierungsprofil auf die Form

$$
M_{\perp}^{\prime}\left(\boldsymbol{r}, \tau_{\mathrm{A}}\right)=\mathrm{i} \gamma M_{z}^{0} \exp \left[-\mathrm{i} \gamma \boldsymbol{G}_{s} \cdot\left(\boldsymbol{r}-\boldsymbol{r}_{0}\right) \frac{\tau_{\mathrm{A}}}{2}\right] \int_{-\tau_{\mathrm{A}} / 2}^{\tau_{\mathrm{A}} / 2} \mathrm{~d} t^{\prime} B_{1}^{\mathrm{e}}\left(t^{\prime}+\frac{\tau_{\mathrm{A}}}{2}\right) \exp \left[\mathrm{i} \gamma \boldsymbol{G}_{s} \cdot\left(\boldsymbol{r}-\boldsymbol{r}_{0}\right) t^{\prime}\right]
$$

proportional zur Fouriertransformierten der Einhüllenden gebracht werden kann, die hier als symmetrisch um $t=\tau_{\mathrm{A}} / 2$ angenommen wurde. Der vor dem Integral stehende räumlich variable Phasenfaktor wird in einer Sequenz meist nach der Anregung durch ein geeignetes Magnetfeld, den sogenannten Rephasierer $\boldsymbol{G}_{\mathrm{r}}$, eliminiert [17, Abschnitt 5.1.4].

\subsubsection{Ortskodierung}

Die Ortskodierung des NMR-Signals erfolgt während der freien Präzession über die Einstellung und Messung definierter Phasenlagen der beitragenden lokalen Magnetisierungskomponenten. Mit Gleichung (2.14) und Definition (2.23) schreibt man

$$
M_{\perp}^{\prime}(\boldsymbol{r}, \boldsymbol{k})=M_{\perp}^{\prime}(\boldsymbol{r}) e^{\mathrm{i} \boldsymbol{k} \cdot \boldsymbol{r}} .
$$

Als Volumenintegral über alle (angeregten) Spins (einer Schicht) erweist sich das in Abhängigkeit von $\boldsymbol{k}$ gemessene Signal

$$
S(\boldsymbol{k})=\int \mathrm{d} \boldsymbol{r} M_{\perp}^{\prime}(\boldsymbol{r}) e^{\mathrm{i} \boldsymbol{k} \cdot \boldsymbol{r}}
$$

als Fouriertransformierte der transversalen Magnetisierungsbeiträge.

Um die Invertierung der orthogonalen Integraltransformation in Gleichung (2.45) durchführen zu können, muß während der Messung der k-Raum abgetastet werden. Die 


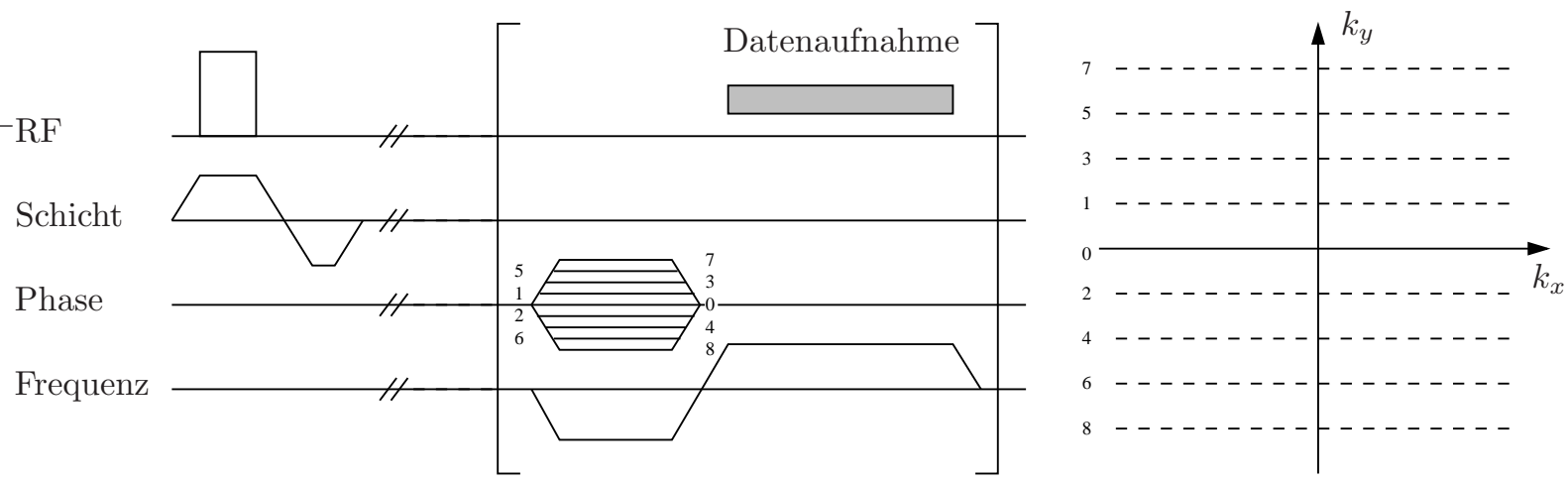

Abbildung 2.6: Fourier-Bildgebung. Der Anregungspuls wird durch einen Schichtgradienten ortsselektiv gemacht. Die dabei erzeugte, unerwünschte Dephasierung in Schichtrichtung erfordert eine Kompensation durch ein definiertes Moment mit invertiertem Vorzeichen. Wie die Präparation der Magnetisierung im weiteren Verlauf erfolgt, ist für jeden Sequenztyp individuell, es sind aber die notwendigen Magnetfelder eingezeichnet, um eine typische Ortskodierung durchzuführen. Der geklammerte Abschnitt wird für jede benötigte k-Raum-Linie einmal und insgesamt $N_{\mathrm{ph}}^{\text {acq }}$-mal durchlaufen. Dabei selektiert man mit der (zur Illustration numerierten) variablen Amplitude des Phasenkodierers die $k_{y}$-Koordinate (mit dem korrespondierenden Index) im Fourier-Raum. Um in $k_{x}$-Richtung eine spiegelsymmetrische Abtastung der gestrichtelt gezeichnete Phasenkodierlinien zu erreichen, wirkt der Frequenzkodierer (Lesegradient) typischerweise zunächst in negativer Richtung. Die für das Beispiel und auch in der Arbeit benutzte, um $k_{y}=0$ alternierende Abdeckung des k-Raums wird im Englischen als „centric reordering “ bezeichnet.

dabei beschriebene Bahnkurve bezeichnet man als k-Raum-Trajektorie. In der einfachen Fourier-Bildgebung liegen die Datenpunkte auf den Schnittpunkten kartesischer Koordinatenlinien. Eine Linie, konventionsgemäß entlang der $k_{x}$-Richtung, wird jeweils während eines Echos aufgenommen. Den beteiligten Gradienten bezeichnet man als Frequenzkodierer. In der Zeit zwischen den Datenaufnahmevorgängen kommt ein „Phasenkodiergradient" zum Einsatz, dessen wechselnde Momente (also seine Zeitintegrale) die $k_{y^{-}}$ Koordinate der Linien bestimmen. Typische Elemente einer Bildgebungsschaltung sind in Abbildung 2.6 dargestellt.

\subsubsection{Sequenzen und Bildkontrast}

Die verschiedenen Echo-Mechanismen aus Abschnitt 2.2 können in Kombination mit ortsaufösenden Gradienten auf vielfältige Weise zu Bildgebungssequenzen zusammengesetzt werden. Das NMR-Experiment wird desweiteren durch viele relativ frei wählbare Größen wie z. B. Flipwinkel, Repetitions- und Echozeiten und nicht zuletzt Diffusionswichtung parametrisiert, so daß ein großer Konfigurationsraum zur Verfügung steht, der in der medizinischen Anwendung die hervorragende Weichteildifferenzierung ermöglicht. Die Sequenz- und Parameterwahl bestimmt dabei auf nichttriviale Weise die Intensitätsverteilung eines Bildes, in dem sich in Abhängigkeit von lokalen $T_{1^{-}}, T_{2}^{(\star)}$ - und Protonendichte-Werten oder auch Diffusionseigenschaften in diesem Sinne homogene Re- 
gionen einer Probe voneinander abheben. Es sind immer mehrere charakteristische Größen in unterschiedlicher Ausprägung für die Intensität eines Bildelements verantwortlich. Man spricht in diesem Zusammenhang von gewichteten Bildern. Für die Diffusionstensorbildgebung sind allerdings nicht absolute Kontraste in Einzelbildern sondern Relationen zwischen Aufnahmen mit unterschiedlichen b-Faktoren entscheidend. Der absolute Wert eines Bildelements ist implementationsabhängig und wird von der Anzahl der Diskretisierungsstufen des Analog-Digital-Wandlers, der das NMR-Signal verarbeitet, bestimmt. Das mittlerweile standardisierte medizinische Datenformat (DICOM) sieht prinzipiell eine Kodierung in 12-Bit vor. Weil die meisten konventionellen Anzeigegeräte digitaler Bilder nur 256 Graustufen darstellen können, muß eine Transferfunktion gewählt werden, die meist aus einer einfachen Stufenfunktion mit linearer Rampe besteht. Der Abszissenbereich W, auf dem die Rampe wirkt, stellt das Intervall dar, in dem einzelne Bildwerte unterschieden werden können. Der Kontrast läßt sich über die Steigung, also die Größe von W und die Position C, den Mittelpunkt von W, variieren. Diesen Vorgang nennt man Fensterung.

\subsubsection{Auflösungsvermögen}

Weil entlang einer Ortskodierungsrichtung nur endlich viele Datenpunkte im Abstand $\Delta k$ bis zu einer Maximalfrequenz $k_{\max }$ aufgenommen werden, läßt sich die Datenmenge in der Form

$$
S(k)=S_{\text {ideal }}(k) \operatorname{Rect}\left(\frac{k}{2 k_{\max }}\right) \sum_{n=-\infty}^{\infty} \delta(k-n \Delta k), \quad k \in \mathbb{R}
$$

mit

$$
\operatorname{Rect}(x):= \begin{cases}1 & ,|x|<1 / 2 \\ 0 & , \text { sonst }\end{cases}
$$

und der Deltafunktion $\delta(x)$ darstellen. Im Bildraum wirkt sich diese Multiplikation des idealen, kontinuierlich und nicht-bandbeschränkt gemessenen Signals, $S_{\text {ideal }}(k)$, mit einer Rechteck- und einer Kammfunktion auf zweierlei Art und Weise aus. Durch die Diskretisierung fließt unter anderem der Signalbeitrag vom Ort $x$ eines Objekts in seiner Abbildung auch in die Bildinformation an dem um

$$
\mathrm{FOV}:=\frac{2 \pi}{\Delta k}
$$

verschobenen Ort mit ein, wie man sich an der Fouriertransformierten der Kamm-Funktion klar macht. Um eine objektgetreue Abbildung zu garantieren, muß also gefordert werden, daß die Probendimensionen das FOV (Field Of View) nicht überschreiten. Ansonsten treten Einfaltungen auf, in der englischen Literatur als „aliasing artefacts“ bekannt. Das ist eine Konsequenz des Abtast-Theorems [17, S.174], das die größte eindeutig rekonstruierbare Längenskala bei gegebenem Abtastintervall $\Delta k$ eigentlich durch $\pi / \Delta k$ 
nach oben beschränkt. Weil aber durch sogenannte Quadraturspulen beide Kanäle des komplexen NMR-Signals und damit ebenfalls der Drehsinn (also auch negative Frequenzen) gemessen werden, kann man Objekte mit Abmessungen innerhalb des Intervalls $[-\pi / \Delta k,+\pi / \Delta k]$ artefaktfrei darstellen.

Die künstliche Frequenzbandbeschränkung der aufgenommenen Daten bewirkt zusammen mit der endlichen Abtastrate eine Faltung des idealen Bildes mit der Fouriertransformierten des Produktes aus Rechteck- und Kammfunktion aus Gleichung (2.46). Weil ein Bildpunkt allgemein durch eine Modulation $f(k)$ im k-Raum über eine typische Länge verschmiert, und dadurch das Auflösungsvermögen beschränkt wird, bezeichnet man ihre Fouriertransformierte $\mathcal{F}\{f\}(x)$ als Punkt-Transfer Funktion. Im NMR-Sprachgebrauch hat sich die englische Abkürzung PSF (Point Spread Function) durchgesetzt. Die Halbwertsbreite $\Delta x$ der PSF kann als Maß für die Aufösung eines NMR-Bildes dienen. In der Fourierbildgebung gilt bei symmetrischer Aufnahme des k-Raums [17, Abschnitt 8.1.2]

$$
\operatorname{PSF}(x)=\operatorname{sinc}\left(k_{\max } x\right)
$$

mit

$$
\Delta x=\frac{\pi}{k_{\max }}
$$

Die Abtastrate des k-Raums in $k_{x}$-Richtung wird rein technisch nur durch die Geschwindigkeit der Aufnahmeelektronik und die verfügbare Gradientenstärke (Schaltzeit und Amplitude) eingeschränkt. Dagegen ist das maximal erzielbare Aufösungsvermögen in Phasenkodierrichtung aus verschiedenen Gründen in der Praxis härteren Randbedingungen unterworfen. Bei Sequenztypen, die nicht auf der Erzeugung eines stationären Zustandes der angeregten Magnetisierung beruhen („steady state“ Sequenzen), wird zur Aufnahme mehrerer Linien der initiale FID durch eine der in Abschnitt 2.2 diskutierten Techniken immer wieder neu zu einem Echo refokussiert. Wegen der Relaxation nimmt die Echointensität, soweit keine geeigneten Vorkehrungen getroffen werden, immer weiter ab, so daß die Akquisitionszeit und damit die Anzahl der Linien durch den Rauschpegel beschränkt ist, und außerdem die zusätzliche Modulation über die damit verbundene Verbreiterung der PSF die effektive Auflösung verschlechtert. Die routinemäßig als Sequenzenparameter angegebene nominelle Größe eines dreidimensionalen Bildelements, auch Voxel genannt, ist durch die Schichtdicke und Gleichung (2.49b) in Phasen- und Frequenzkodierrichtung determiniert. 


\section{KAPITEL 3}

\section{Diffusionstensorbildgebung mit DW-SSSTEAM}

\subsection{Berechnung des Diffusionstensors}

Gesetzt die Gültigkeit einiger Voraussetzungen der Onsagerschen Reziprozitätsbeziehungen $[30,31]$ der Nichtgleichgewichtsthermodynamik ist der Diffusionstensor symmetrisch. Seine Matrizendarstellung besitzt deshalb nur sechs unabhängige Elemente. Theoretisch läßt sich $\mathbf{D}$ daher in einem NMR-Experiment bei gegebenem b-Wert (2.27) aus dem jeweiligen Signalabfall für $N_{\mathrm{G}}=6$ in nicht kollineare Gradientenrichtungen $\boldsymbol{g}_{i}=\boldsymbol{G}_{i} / G_{i}$ geschaltete Diffusionskodierer und aus einer zusätzlichen, ungewichteten Messung bestimmen. Dazu ist das System

$$
\ln \left(\frac{S_{\boldsymbol{G}_{i}}}{S_{\mathbf{0}}}\right)=b \boldsymbol{g}_{i}^{\mathrm{T}} \mathbf{D} \boldsymbol{g}_{i} \quad i=1, \ldots, N_{\mathrm{G}}
$$

von $N_{\mathrm{G}}$ linear unabhängigen Gleichungen in den Unbekannten $\left\{D_{i j}\right\}$ zu lösen [15]. In der Praxis erweist es sich wegen des Meßrauschens als sinnvoll, zur zuverlässigen Schätzung von D bei beliebig orientierten Diffusionsellipsoiden mehr als sechs Diffusionsrichtungen zu verwenden, um eine bessere numerische Konditionierung zu erreichen. Im allgemeinen Fall werden $N_{\mathrm{G}} \geq 6$ Richtungen und $N_{\mathrm{b}} \geq 1$ von Null verschiedene b-Werte benutzt. Das Gleichungssystem (3.1) ist dann überbestimmt. Definiert man den Vektor

$$
\boldsymbol{v}:=\left(D_{x x}, D_{y y}, D_{z z}, D_{x y}, D_{x z}, D_{y z}, \ln \left(S_{0}\right)\right)^{\mathrm{T}},
$$

wobei $S_{0}$ hier als zusätzlicher freier Parameter für den ungewichteten Signalwert zu interpretieren ist, und faßt die $N_{\mathrm{G}} \cdot N_{\mathrm{b}}+1$ logarithmierten Signalwerte in einem Vektor $\boldsymbol{a}$ zusammen, so kann man das Problem auf die Form

$$
a=\mathrm{B} v
$$


mit der Matrix $\mathbf{B} \in \mathbb{R}^{\left(N_{\mathrm{G}} \cdot N_{\mathrm{b}}+1\right) \times 7}$ bringen. Diese Gleichung läßt sich mit dem sogenannten Moore-Penrose- oder auch Pseudo-Inversen [32, Abschnitt 3.3]

$$
\mathbf{B}_{\mathrm{MP}}^{-1}:=\left(\mathbf{B}^{\mathrm{T}} \mathbf{B}\right)^{-1}\left(\mathbf{B}^{\mathrm{T}}\right)
$$

in dem Sinne gemäß $\boldsymbol{v}_{\mathrm{opt}}=\mathbf{B}_{\mathrm{MP}}^{-1} \boldsymbol{a}$ eindeutig und optimal nach dem Vektor $\boldsymbol{v}$ auflösen, als daß dieser die Forderung des Gaußschen Ausgleichsprinzips

$$
\mathcal{R}:=\|\boldsymbol{a}-\mathbf{B} \boldsymbol{v}\|=\min !
$$

für das Residuum $\mathcal{R}$ erfüllt. Zur Berücksichtigung des Rauschens sollte im allgemeinen der gewichtete Ausdruck $\left\|\boldsymbol{\Psi}^{-1 / 2}(\boldsymbol{a}-\mathbf{B} \boldsymbol{v})\right\|$ minimiert werden. Unter der Annahme, daß alle Einzelmessungen statistisch unabhängig voneinander erfolgen, ist die Matrix $\boldsymbol{\Psi}^{-1}$ diagonal, und ihre Elemente sind Funktionen der Standardabweichungen $\sigma_{i}$ des Rauschens auf der $i$-ten Einzelmessung. Wegen der Logarithmierung der Signalgleichungen gilt aufgrund der Fehlerfortpflanzung

$$
\left(\boldsymbol{\Psi}^{-1}\right)_{i i}=\frac{a_{i}^{2}}{\sigma_{i}^{2}} .
$$

Gängigerweise nimmt man außerdem an, daß die Rauschcharakteristik für alle Messungen gleich ist und setzt $\sigma_{i} \equiv 1 \forall i$. Für die Berechnung des Moore-Penrose-Inversen kann man die Singulärwertzerlegung, SVD (Singular Value Decomposition) [32, Abschnitt 5.4], einsetzen, ein Algorithmus, der auch dann numerische Stabilität verspricht, wenn B singulär ist, also linear abhängige Zeilen besitzt. Das ist dann der Fall, wenn bei Verwendung des Gradienten $\boldsymbol{G}$ auch der Diffusionsabfall in Richtung $-\boldsymbol{G}$ bestimmt wird. Alternativ läßt sich $\boldsymbol{v}_{\text {opt }}$ auch als Lösung eines quadratischen Optimierungsproblems auffassen und mit nichtlinearer Regression bestimmen, z. B. mit Hilfe des Levenberg-Marquard-Algorithmus [32, Abschnitt 4.3] durch die Anpassung der parametrisierten Funktion

$$
S_{\boldsymbol{G}_{i}}=S_{0} \exp \left[-\sum_{j, k=1}^{3} b_{j k} D_{j k}\right]
$$

an die untransformierten Signalintensitäten $S_{\boldsymbol{G}_{i}}$ und $S_{\boldsymbol{G}=\mathbf{0}}$.

\subsection{Charakterisierung und Quantifizierung von DTI-Daten}

Nachdem man durch Kombination der Diffusionssensibilisierung mit einem anschließendem Bildgebungsteil und der Verarbeitung der Daten mit den eben ausgeführten Methoden eine räumlich aufgelöste Schätzung des Diffusionstensorfeldes in jedem Bildpunkt in der Hand hat, will man daraus sinnvolle Informationen extrahieren. Von der Vielzahl der vorgeschlagenen Meßgrößen werden hier nur die gängigsten vorgestellt. Eine tiefergehende Diskussion auch im Hinblick auf das Kontrast-zu-Rausch-Verhältnis und Verteilungsfunktionen findet man in [33, 34, 35, 36]. 
Wegen seiner Symmetrie ist $\mathbf{D}$ diagonalisierbar und besitzt drei zueinander orthogonale Eigenvektoren $\left\{\boldsymbol{\epsilon}_{i}\right\}$ mit den dazugehörigen Eigenwerten $\lambda_{1} \geq \lambda_{2} \geq \lambda_{3}$, den Diffusivitäten in Richtung der Hauptachsen des Tensors

$$
\begin{aligned}
\mathbf{D} \boldsymbol{\epsilon}_{i} & =\lambda_{i} \boldsymbol{\epsilon}_{i} \quad i \in\{1,2,3\} \\
\boldsymbol{\epsilon}_{i} \cdot \boldsymbol{\epsilon}_{j} & =\delta_{i j} .
\end{aligned}
$$

Aus physikalischen Gründen muß D positiv semi-definit sein, d.h. $\lambda_{i} \geq 0$. Ansonsten könnte sich ein (Teilchen-) Fluß entgegen der Richtung einer verallgemeinerten Kraft in der Thermodynamik ausbilden.

Eine einfache skalare Größe, die es erlaubt, die mittlere Behinderung der Wassermoleküle durch mikroskopische Barrieren in Relation zur freier Diffusion zu bewerten, ist der ADC (Apparent Diffusion Coefficient)

$$
\mathrm{ADC}:=\frac{1}{3} \operatorname{tr}(\mathbf{D})
$$

Abbildung 3.1 (a) zeigt beispielsweise erwartungsgemäß, daß die Beweglichkeit in den Ventrikeln des Gehirns, das sind mit Cerebrospinalflüssigkeit (Liquor) gefüllte Kavitäten, erheblich größer ist als im umliegenden Gewebe. Der ADC hat in der klinischen Anwendung eine große Bedeutung, weil er als ein Frühindikator in der Schlaganfalldiagnostik dient. Bei diesem Krankheitsbild machen sich Gewebe-Anomalien in ischämischen Regionen auf entsprechenden Karten in Form von hypointensen Voxeln bzw. auf diffusionsgewichteten Bildern durch hyperintense Bildpunkte schnell bemerk- und lokalisierbar, wohingegen andere NMR-Kontrastmechanismen erst im späteren Verlauf ansprechen. Die Kernspin-Untersuchung kann deshalb in diesem Fall die konventionell verwendete, mit einer Strahlenbelastung verbundene Computertomographie ersetzen.

Über die Struktur des Spektrums von D lassen sich mit Hilfe abgeleiteter Größen Aussagen über den Organisationsgrad von Gewebe absolut quantifizieren. Als Maß für die Gerichtetheit dient z. B. die fraktionelle Anisotropie [34]

$$
\mathrm{FA}:=\sqrt{\frac{3}{2} \frac{\sum_{i=1}^{3}\left(\lambda_{i}-\mathrm{ADC}\right)^{2}}{\sum_{i=1}^{3} \lambda_{i}^{2}}}
$$

deren Wertebereich auf das Intervall $[0,1]$ eingeschränkt ist. Die beiden Enden dieser Skala zeigen jeweils Isotropie $(\mathrm{FA}=0)$ und höchstmögliche Richtungsabhängigkeit $(\mathrm{FA}=1)$ der Diffusion an. Wie in Abbildung 3.1 (a) deutlich wird, erlaubt diese Größe eine sinnvolle Differenzierung grauer und weißer Hirnsubstanz. Weil es sich bei der fraktionellen Anisotropie bei festen Sequenzparametern um eine absolute Größe handelt, ist sie nicht wie die Intensitäten in $T_{1}$ - oder $T_{2}$-gewichteten Bildern vom Sensitivitätsprofil der Aufnahmespulen abhängig, und man kann einzelne Voxel anhand von definierten Wertebereichen direkt unterschiedlichen Gewebsarten zuordnen. Die fraktionelle Anisotropie wird inzwischen in vielen klinischen Studien vor allem zur Untersuchung neurodegenerativer 

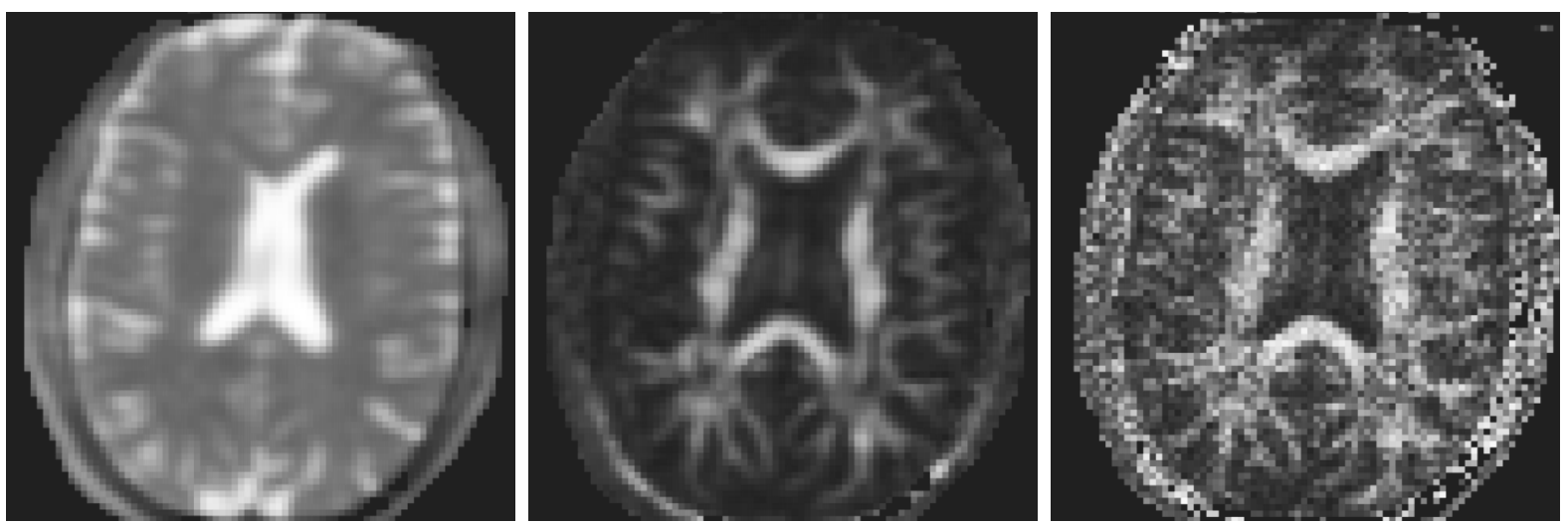

(a) Quantifizierung von DTI: (links) ADC, (Mitte) FA, (rechts) FA-Karte ohne Tiefpassfilterung.
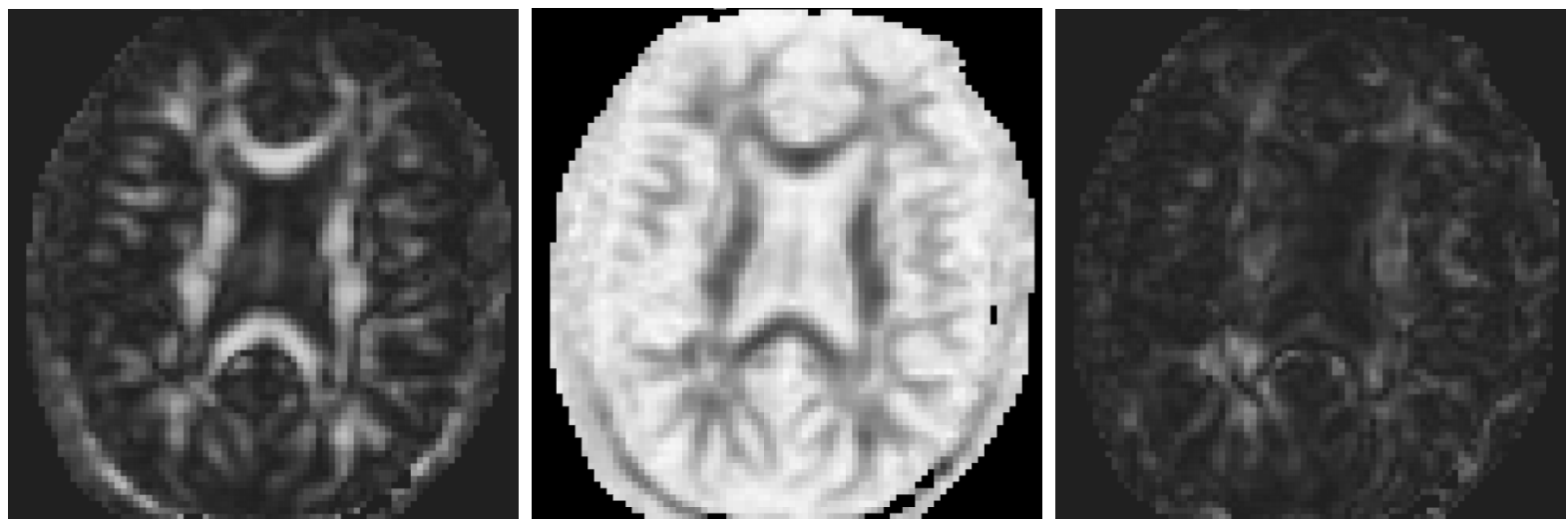

(b) Geometrische Charakterisierung des Diffusionstensor: (links) $c_{1}$, (Mitte) $c_{\mathrm{s}}$, (rechts) $c_{\mathrm{p}}$.
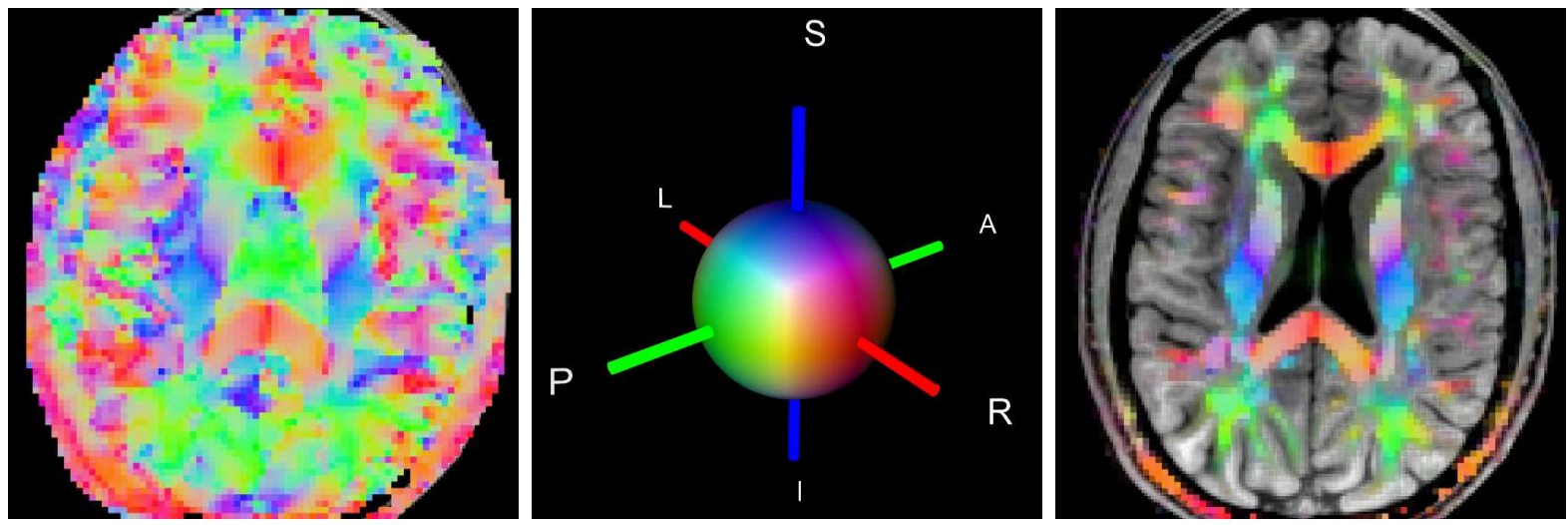

(c) Darstellung der Faserorientierung: (links) farbkodierte MDD-Karte. (Mitte) Farbkodierungskonvention. Für die typische Positionierung finden die Scanner-Koordinatenachsen, die das Referenzsystem für die Farbkodierung darstellen, ihre Entsprechung im Probandenkoordinatensystem in den Richtungen A-P: Anterior $\leftrightarrow$ Posterior. R-L: Rechts $\leftrightarrow$ Links. I-S: Inferior $\leftrightarrow$ Superior. (rechts) Farbkodierte MDD-Darstellung mit zusätzlicher Intensitätswichtung und Transparenz als Funktionen der Anisotropie über einer $T_{1}$-gewichteten anatomischen Aufnahme. 
Erkrankungen verwendet.

Die anschauliche Darstellung des Diffusionstensors mit Hilfe des Diffusionsellipsoids, dessen Dimensionen und Hauptachsen durch die Eigenwerte und -vektoren von D bestimmt sind, und das eine Niveaufläche der Verschiebungswahrscheinlichkeit eines Teilchens darstellt, legt eine allgemeine geometrische Klassifizierung nahe [37]. Während der Informationsgehalt des Linearitätsindexes $c_{1}$ und des Spherizitätsindexes $c_{\mathrm{s}}$, also die Ähnlichkeit des Ellipsoids mit einer Zigarren- bzw. Kugelform

$$
\begin{aligned}
& c_{1}:=\frac{\lambda_{1}-\lambda_{2}}{\lambda_{1}} \\
& c_{\mathrm{s}}:=\frac{\lambda_{3}}{\lambda_{1}},
\end{aligned}
$$

weitgehend durch die fraktionelle Anisotropie abgedeckt wird, beschreibt

$$
c_{\mathrm{p}}:=\frac{\lambda_{2}-\lambda_{3}}{\lambda_{1}}
$$

die Ausgeprägtheit der Planarität oder Pfannkuchenförmigkeit (siehe Abbildung 3.1 (b)). Ein hoher $c_{\mathrm{p}}$-Wert kann als Indikator für Voxel mit sich kreuzenden Fasern dienen.

Die Aussagekraft der Charakterisierung von quasifreier Diffusion mit den beschriebenen Größen wird von der Tatsache eingeschränkt, daß eine Vielzahl unterschiedlicher mikroskopischer Geometrien die gleichen Werte erzeugen. Eine Bewertung solcher Karten kann somit nur empirisch erfolgen. Außerdem muß man beachten, daß die berechneten Maße im allgemeinen Funktionen von $\delta_{\mathrm{D}}$ und $\Delta_{\mathrm{D}}$ sind. Allerdings zeigte eine Untersuchung von Clark et al. [38] innerhalb der typischerweise verwendeten Intervalle eine weitgehende Unabhängigkeit der untersuchten Indizes von den verwendeten Diffusionszeiten.

Im Gegensatz zu den vorgestellten skalaren Maßen steht bei der Rekonstruktion der makroskopischen dreidimensionalen Geometrie von gerichtetem Gewebe die vektorielle Komponente der Eigenstruktur des Diffusionstensors im Vordergrund. Wie bereits in Abschnitt 2.3.2 erwähnt wurde, orientiert sich $\boldsymbol{\epsilon}_{1}$ parallel zur Faserrichtung, wenn eine solche sinnvoll definiert ist. Bereits mit relativ einfachen Algorithmen kann man deshalb z. B. langreichweitige axonale Verbindungen in der weißen Hirnsubstanz oder Muskelstränge grob verfolgen und visualisieren (,fiber tracking“), eine Thematik, der Kapitel 5 gewidmet ist. Allerdings läßt sich in speziellen zweidimensionalen Karten schon mit dem bloßen Auge ein erster Eindruck von der Topologie gewinnen. Besonders anschaulich ist die Farbkodierung von $\boldsymbol{\epsilon}_{1}$ (Abbildung 3.1 (c)). Im Koordinatensystem des MRT-Scanners entspricht rot einer rechts $\leftrightarrow$ links-Verbindung $(x)$, grün einer Orientierung entlang des statischen Magnetfeldes $(z)$ und blau der komplementären Richtung

\footnotetext{
Abbildung 3.1 (gegenüberliegende Seite): Quantifizierung und zweidimensionale Visualisierung des Diffusionstensorfeldes. Die Daten wurden, soweit nicht explizit anders vermerkt, vor der Schätzung von D mit einem Gauß-Filter geglättet.
} 

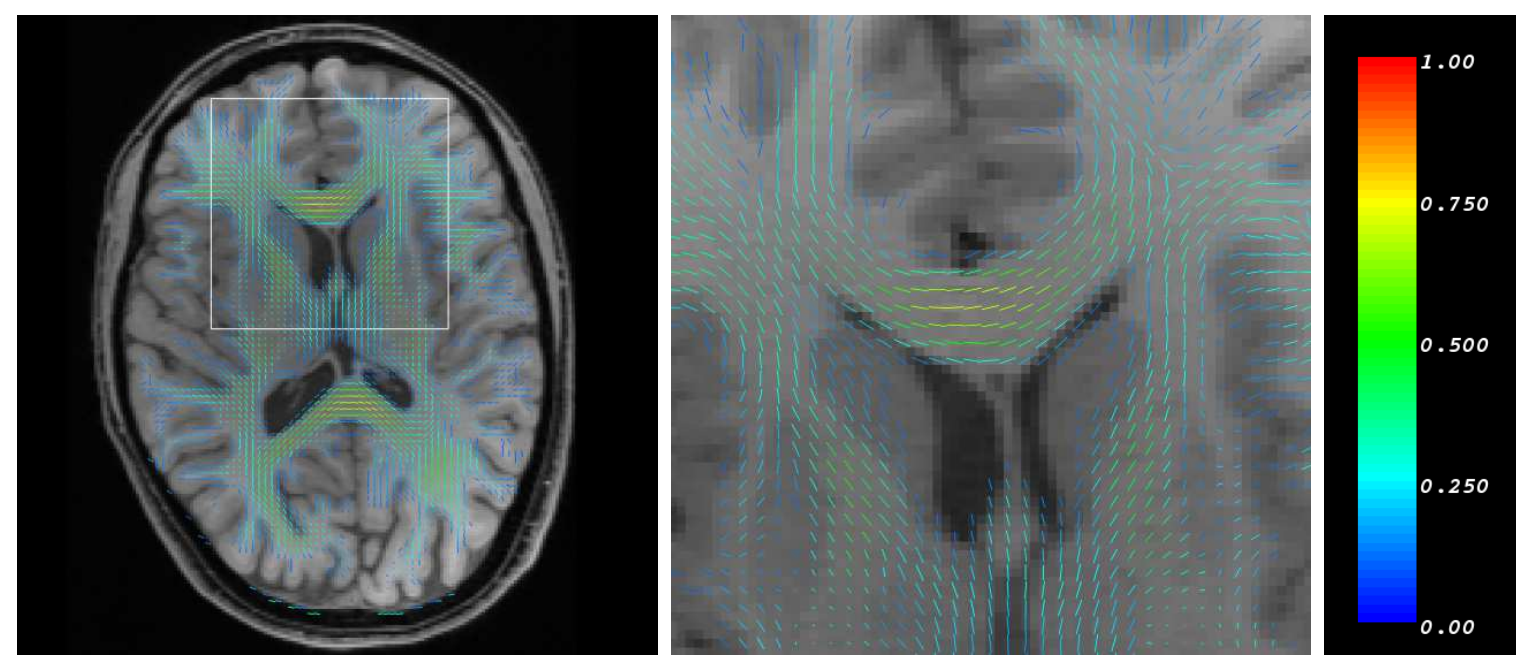

Abbildung 3.2: MDD-Glyphendarstellung. (links) Visualisierung der Hauptdiffusionsrichtung mit Linienelementen, (Mitte) Vergrößerung der im linken Bild eingezeichneten Region, (rechts) die Farbskala bildet den lokalen Anisotropiewert (FA) ab.

von oben nach unten $(y)$. Bei der für craniale Untersuchungen üblicherweise verwendeten Lagerung der Probanden ergibt sich im Körperkoordinatensystem folgende Entsprechung: rot $=$ rechts $\leftrightarrow$ links, grün $=$ anterior $\leftrightarrow$ posterior und blau $=$ inferior $\leftrightarrow$ superior. Ergänzend zeigt Abbildung 3.2 eine Glyphendarstellung der Projektion der Hauptdiffusionsrichtung $\boldsymbol{e}_{1}$, MDD (Main Diffusion Direction), auf die Ebene des hinterlegten anatomischen Schnittbildes eines menschlichen Gehirns.

\subsection{Artefakte, Randbedingungen und Sequenz-Konzeption}

Bevor die in der vorliegenden Arbeit zur Diffusionstensorbildgebung verwendete DWSSSTEAM-Sequenz vorgestellt wird, ist es an dieser Stelle angebracht, einige Bemerkungen zu den potentiellen Fehlerquellen und Randbedingungen einer Diffusionstensormessung anzubringen, die den Rahmen für die Konzeption eines solchen Experimentes vorgeben.

\subsubsection{Systematische Fehler}

Das Spektrum der systematischen Fehler läßt sich grob in zwei nicht gänzlich unabhängige Gruppen gliedern, Bildartefakte und Auswertefehler.

\section{Bildartefakte}

Wie bereits bei der Erläuterung des Gradientenechos ausgeführt, leiden Sequenzen, die auf diesem Signalmechanismus basieren, an geometrischen Abbildungsfehlern und Intensitätsartefakten aufgrund schwer kontrollierbarer statischer und transienter Magnetfeldvariationen, die einerseits durch Suszeptibilitätsunterschiede und $\boldsymbol{B}_{0}$-Inhomogenitäten 
und andererseits durch induzierte Wirbelströme ausgelöst werden. Diese Effekte sind insbesondere bei der für die Diffusionstensorbildgebung hauptsächlich eingesetzten EPIBildgebungssequenz (Echo Planar Imaging) [11], die sämtliche k-Raum-Linien eines Bildes nach einer einzigen RF-Anregung aus einer Abfolge von Gradientenechos konstruiert, stark ausgeprägt. Wirbelströme treten als Folge rascher Gradientenschaltungen sowohl in bildgebenden Spulen und anderen elektrisch leitenden Bestandteilen des NMR-Scanners als auch im Versuchsobjekt selber auf. Ihre Entstehung wird durch die Tatsache begünstigt, daß die diffusionskodierenden Pulse wegen des raschen $T_{2}$-Abfalls und der benötigten hohen b-Werte sehr schnell auf große Amplituden gefahren werden müssen. Weil außerdem die Richtung der entsprechenden Gradienten bei einer Tensor-Messung variiert, sind die geometrischen Verzerrungen für unterschiedlich gewichtete Aufnahmen derselben Schicht nicht gleich.

Ein geometrisches Abbildungsproblem anderer Art ergibt sich dadurch, daß die Wasserstoffkerne in Fettgewebe eine geringfügig andere Larmor-Frequenz als in Wasser besitzen. Durch fehlerhafte Ortskodierung erscheint deswegen bei zu geringer Aufnahmebandbreite ein entlang der Frequenzkodierrichtung verschobenes Teilbild. Dieser Effekt ist in der englischen Literatur als CS-Artefakt (Chemical Shift) bekannt. Um das Übel an der Wurzel zu packen, kann man z.B. vor der eigentlichen Sequenz sogenannte fettsättigende Anregungspulse einsetzen. Außerdem besteht die Möglichkeit, die Auswirkungen der Frequenzabweichung zu minimieren, indem man die Aufnahmebandbreite pro Pixel durch Einsatz eines starken Auslesegradienten so weit vergrößert, daß die räumliche Verschiebung in der Abbildung kleiner als die Dimension eines Bildelements ausfällt. Das impliziert aber nach Gleichung (3.12) eine Einbuße beim Signal-zu-Rausch-Verhältnis.

Sequenzbedingte Bildartefakte können auch dadurch entstehen, daß unerwünschte Anteile der transversalen Magnetisierung entweder als FIDs oder als (höhere) Echos während der Datenaufnahme zum NMR-Signal beitragen. Ihr Einfluß kann sich in Form von periodischen Intensitätsfehlern und Geisterbildern manifestieren. Davon sind besonders reine RF-Echo-Sequenzen betroffen. Man versucht dem Problem dadurch beizukommen, daß man zusätzliche Gradienten paarweise symmetrisch zu bestimmten Anregungspulsen oder einzeln direkt danach schaltet, um mit diesen im MR-Jargon als Crusher und Spoiler bezeichneten Magnetfeldern störende Echopfade zu unterdrücken (siehe [39]).

Neben den beschriebenen hardware- und sequenzspezifischen Fehlern birgt auch das Versuchsobjekt bzw. -subjekt selbst eine Fülle von Artefaktquellen in sich. Obwohl bei in-vivo Messungen an menschlichen Probanden diese mit Kissen geeignet fixiert und vor Beginn entsprechend instruiert werden, ist es während eines 15-30-minütigen Diffusionstensorexperiments natürlich nicht ausgeschlossen, daß makroskopische Bewegungen von der Größenordnung der Voxeldimensionen auftreten, wie die Beispiele in Abbildung 3.3 verdeutlichen. Bei einer Kopfuntersuchung sind außerdem Augen-Motilität und die Verwendung des Schluckapparates nahezu unvermeidbar. Je nachdem wann die Störung im Sequenzablauf erfolgt, manifestiert sie sich entweder deutlich sichtbar in den Einzelbildern als Verwaschung bzw. Geisterbild oder subtiler in Verschiebungen aufeinanderfolgender Schichten.

Eine spezielle Klasse von Bewegungsartefakten bilden Hirnpulsationen im Bereich der Ventrikel, die zeitlich mit dem Herzschlag korrelieren, mit Gewebsstauchungen von 

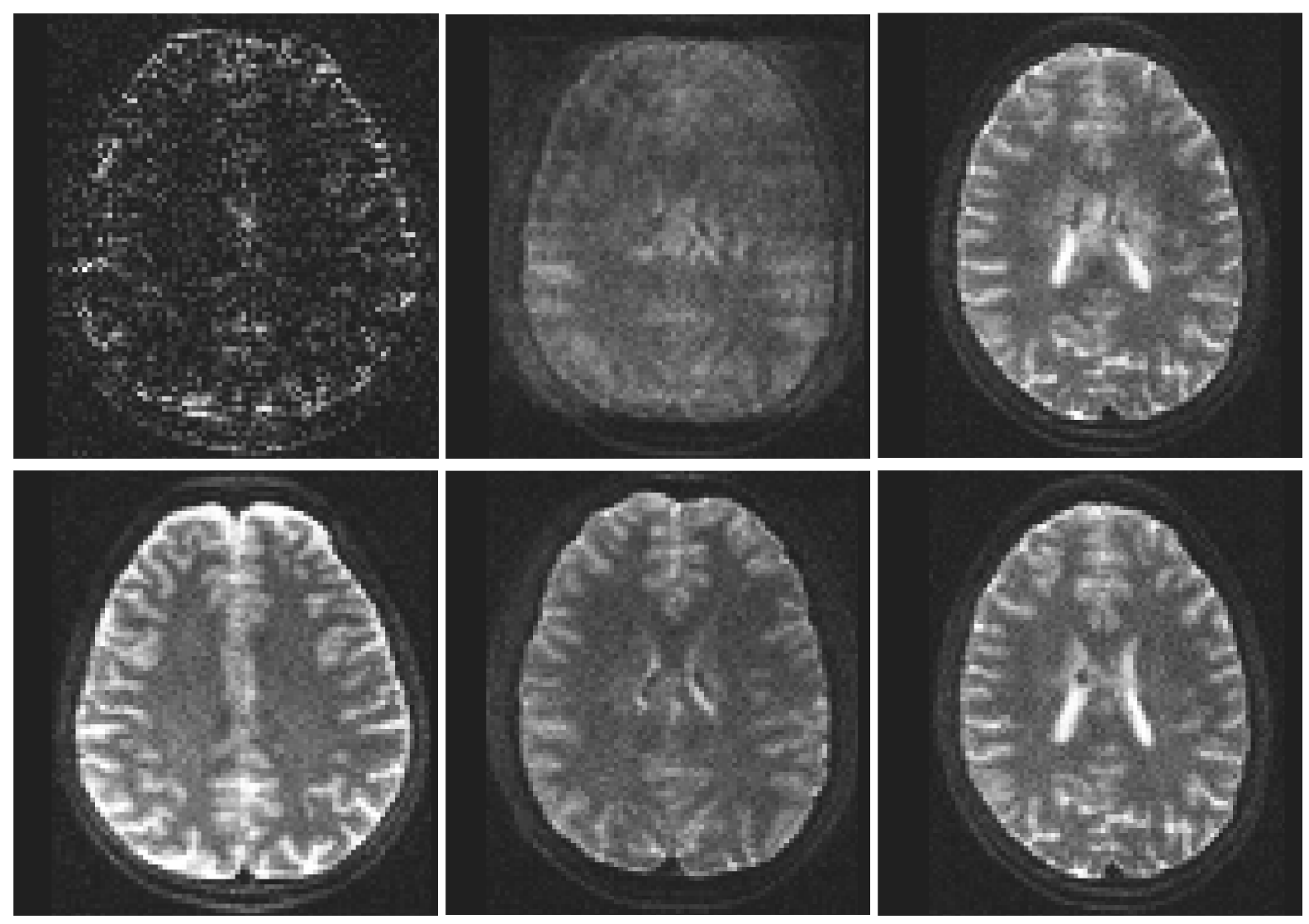

Abbildung 3.3: Ausprägungen von Bewegungsartefakten bei in-vivo Messungen. In den Spalten ist jeweils oben das Artefakt und unten eine nicht (oder gering) betroffene Referenzaufnahme zu sehen. (links) Makroskopische Bewegung zwischen Repetitionen: das obere Bild zeigt die Differenz zweier zu Beginn und am Ende einer ca. halbstündigen Sitzung angefertigter nicht diffusionsgewichteter Aufnahmen. (Mitte) Bewegung während der Schichtaufnahme. Der Proband wurde gebeten, bei der Messung zu husten. (rechts) Intensitäts- und Geometrieartefakte durch Hirnpulsation im Bereich der Ventrikel.

0.1-0.5 mm [40] verbunden sind, und deren Auftreten sich der aktiven Kontrolle des Probanden entzieht. Als Konsequenz beobachtet man in NMR-Bildern Geometrie- und Intensitätsfehler. Eine Möglichkeit, ihnen zu begegnen, ist die Synchronisation der Meßsequenz mit diesen Zyklen z. B. mithilfe von EKG-Daten (Elektrokardiogramm) [41, 42] oder aber man identifiziert und vernachlässigt die betroffenen Bilder [43].

Bewegungsartefakte innerhalb einer Einzelaufnahme können durch eine möglichst kleine Bildakquisitionszeit minimiert werden. Allerdings besteht wegen der schichtweise abweichenden Orientierung beeinträchtigter Messungen immer noch das Problem, daß sich die Schnittbilder nicht auf triviale Weise zu einem dreidimensionalen Datensatz zusammenfügen lassen, wenn die Bewegung nicht parallel zur Schnittebene erfolgt ist.

Zu guter Letzt treten bedingt durch große Feldstärkenänderungen in den Gradientenspulen des NMR-Gerätes starke mechanische Belastungen auf, die sich über Erschütterungen des Gerätes auch auf die Versuchsperson übertragen. Die dadurch provozierten 
Auslenkungen aus der Ruhelage beschränken fundamental das Aufösungsvermögen.

Auch bei auf den ersten Blick fehlerarmen Bildern sollte man sich darüber im klaren sein, daß allein die begrenzte Auflösung ein unvermeidbares Artefakt darstellt. Sind in einem Voxel mehrere unterschiedliche Gewebsarten enthalten, ist die Bildintensität in diesem Punkt eine Mischung der entsprechenden Signalanteile. Dieser Partialvolumeneffekt kann verdeckt dadurch verschärft werden, daß die Breite der Punkt-Transfer Funktion durch übermäßig starken Signalabfall über dem k-Raum die durch die FourierAuflösung (2.49b) gegebene Voxel-Abmessung übersteigt.

\section{Auswertefehler}

Die meisten Bildbeeinträchtigungen übersetzen sich unbehandelt naturgemäß in Auswertefehler. Geometrische Verzerrungen verfälschen die Topologie des Gehirns und können bei der Faserverfolgung falsch positive Verbindungen bestimmter Regionen vorgaukeln. Besonders problematisch sind solche Abbildungsfehler, wenn sie wie im Fall der Wirbelstromartefakte inkonsistent auf ein und dieselbe Aufnahmeschicht wirken, weil dadurch bei der numerischen Schätzung des Diffusionstensors räumlich nicht zusammengehörige Voxeldaten miteinander verrechnet werden. Derselbe Effekt tritt auch als Folge von Bewegungen der Versuchsperson während der Messung auf. Vielfach versucht man, topologisch inkorrekte Daten durch Transformation auf die Gestalt nicht davon betroffener anatomischer Referenzaufnahmen zu bringen. Sind allerdings bei der Bildgebung die Informationen verschiedener Ortskoordinaten (nichtaffin) auf ein einzelnes Volumenelement abgebildet worden, muß das Resultat unbefriedigend bleiben.

Neben der bereits beschriebenen Wirkung beeinträchtigen Wirbelströme und Suszeptibilitätseffekte auch die b-Matrix und machen sie in den betroffenen Regionen ortsabhängig [44]. Außerdem ist theoretisch auch der Beitrag der Bildgebungsgradienten zur b-Matrix zu beachten, die in Gleichung (2.26) in gemischten Produkten mit den eigentlichen Diffusionsgradienten auftauchen und deshalb betragsmäßig nicht vernachlässigbar sind. Eine von Neeman [45] propagierte einfache Korrekturmethode zur Eliminierung dieser Kreuzterme besteht darin, mit jeder Kodierungsrichtung $\boldsymbol{G}$ auch die Gegenrichtung $-G$ zu messen und das geometrische Mittel dieser Aufnahmen zu bilden. Die äquivalenten störenden Beiträge in den beiden Bildern haben dann nämlich wegen der konstanten Richtung der beteiligten Bildgebungsgradienten genau das entgegengesetzte Vorzeichen und heben sich deshalb im Exponenten des gemittelten Signals (siehe Gleichung (2.25b)) weg. Aus offensichtlichen Gründen sind auch hardwarebedingte unerwünschte Magnetfeldvariationen wie z. B. Inhomogenitäten des statischen Magnetfeldes $\boldsymbol{B}_{0}$ oder nichtlineare Gradienten Gift sowohl für die Bildgebung als auch für die exakte Applikation der Diffusionswichtung.

Partialvolumeneffekte machen sich in der Diffusionstensorberechnung in jedem Fall quantitativ bemerkbar. Beinhaltet ein Voxel allerdings Axone bloß eines Faserbündels und zusätzlich nur isotropes Gewebe, so wird sich das einzig auf die skalaren Größen, nicht aber auf die Bestimmung der Hauptdiffusionsrichtung negativ auswirken. Dagegen kann ein Bildelement, in dem sich mehrere Fasern kreuzen, nicht mehr adäquat mit dem Diffusionstensormodell beschrieben werden. Modell- und damit Auswertefehler schleichen 
sich, wie in Abschnitt 2.3.4 angedeutet, auch dann ein, wenn die Meßparameter, hier speziell die b-Werte, den Gültigkeitsbereich verlassen. Als empirische Obergrenze hat sich in Diffusionstensormessungen für die Stärke der Diffusionswichtung $b \approx 1000 \mathrm{~s} / \mathrm{mm}^{2}$ eingebürgert.

\subsubsection{Rauschen und das SNR}

Während der Datenakquisition stört thermisches und bei in-vivo-Messungen auch physiologisches Rauschen das wahre Signal. Sein als additiv angenommener Einfluß auf die beiden Aufnahmekanäle für die gemessene komplexe Magnetisierung $M_{\perp}$ wird jeweils durch einen stochastischen Gaußschen Prozeß mit Mittelwert Null und Varianz $\sigma^{2}$ modelliert. Das Signal-zu-Rausch-Verhältnis, SNR (Signal-to-Noise Ratio), ist über den Quotienten aus Signalstärke und der Standardabweichung des Rauschens definiert und stellt einen konventionell verwendeten Qualitätsmaßstab dar. Es läßt sich mit Hilfe von Mittelungen über $N_{\text {av }}$ Datensätze verbessern. Weil der ungestörte Signalanteil dabei kohärent aufaddiert wird, die Rauschamplitude dagegen einen „random walk“ beschreibt, verbessert sich das SNR theoretisch mit der Wurzel der Mittelungszahl. Die Wahl der Meßparameter beeinflußt allgemein die Güte der Daten. Mit dem Voxelvolumen $V_{\text {voxel }}$, der Empfängerbandbreite $B W$ und der Anzahl $N_{\mathrm{ph}}^{\text {acq }}$ der (akquirierten) Phasenkodierlinien gilt (siehe $[46, \mathrm{~S} .13-14])$

$$
\mathrm{SNR} \propto V_{\text {voxel }} \sqrt{\frac{N_{\mathrm{ph}}^{\mathrm{acq}} N_{\mathrm{av}}}{B W}} .
$$

In der NMR-Bildgebung bezieht man das Signal-zu-Rausch-Verhältnis auf ein bestimmtes charakteristisches Gewebe und gibt als Schätzwert häufig den Intensitätsmittelwert einer Region relativ hoher Homogenität im Versuchsobjekt geteilt durch die Standardabweichung des Mittelwertes einer signal- und artefaktfreien Region außerhalb der Probe. Um Phasenfehler zu eliminieren, die wegen der starken Gradienten schon durch geringfügige Bewegungen des Versuchsobjekts während der Diffusionskodierung auftreten und bei Bildmittelung zu Signalauslöschung führen, arbeitet man aber in der Diffusionsbildgebung meistens mit dem Absolutbetrag, der Magnitude, der komplexen Daten. Wegen dieser nichtlinearen Transformation folgt die Verteilung $p_{\mathrm{I}}(I)$ eines Voxelwertes nicht mehr der Gauß- sondern der Rice-Statistik [47], die nur für große wahre Bild-Werte durch eine Glockenkurve mit Mittelwert $\sqrt{I_{0}^{2}+\sigma^{2}}$ und Varianz $\sigma^{2}$ approximiert werden kann. Dabei ist $I$ die gemessene Bildintensität in Anwesenheit von Rauschen und $I_{0}$ der wahre Wert. In signalfreien Regionen tritt als Grenzfall die Rayleigh-Verteilung

$$
p_{\mathrm{I}}(I)=\frac{I}{\sigma^{2}} \exp \left[-\frac{I^{2}}{2 \sigma^{2}}\right]
$$


mit

$$
\begin{aligned}
\langle I\rangle & =\sigma \sqrt{\frac{\pi}{2}} \\
\sigma_{\mathrm{I}}^{2} & =\left(2-\frac{\pi}{2}\right) \sigma^{2}
\end{aligned}
$$

auf. Deshalb wird die oben genannte SNR-Meßmethode systematisch verfälscht. Mit Hilfe der Beziehungen (3.13b) und (3.13c) ist es aber prinzipiell möglich, das Signal-zu-RauschVerhältnis eines Magnitudenbildes konsistent zu schätzen. Wird dieser Weg gewählt, darf man bei entsprechend gemittelten Aufnahmen allerdings nur Gleichung (3.13b) verwenden, da sich die Standardabweichung im Gegensatz zum Erwartungswert verändert. Wichtig ist, daß die Mittelung von Magnitudenbildern zwar zur Verbesserung der Mittelwerte beiträgt aber nicht im eigentlichen Sinne das Signal-zu-Rausch-Verhältnis erhöht, weil der Rauschuntergrund, der Mittelwert aus Gleichung (3.13b), gleich bleibt. Das ist künstlich nur durch die Addition der komplexen Daten möglich, denn die so berechneten Bilder sind dann nicht mehr von einer Messung mit einem um den Faktor $1 / \sqrt{N_{\mathrm{av}}}$ verringertem $\sigma \mathrm{zu}$ unterscheiden.

Da der Rauschuntergrund in vielen nicht-quantitativen NMR-Messungen vernachlässigbar ist, und er dann nicht den visuellen Eindruck der Bilder beeinträchtigt, wird man manchmal mißverständlicherweise mit der Aussage konfrontiert, daß auch Magnitudenmittelung das SNR verbessert. Das ist, wie oben erläutert, im strengen Sinne nicht vollständig korrekt [48] insbesondere, wenn das SNR als Qualitätsmerkmal signalschwacher, diffusionsgewichteter Bilder verwendet wird. Für die pragmatische Charakterisierung eines Magnitudenbildes benötigt man also den Signalwert der Zielregion, die Standardabweichung seiner Verteilung und zusätzlich den Rauschuntergrund.

Die Auswirkungen des Rauschens auf die Diffusionstensorbildgebung sind vielschichtig. Zunächst einmal bewirkt es eine Verbreiterung des Spektrums von D [49], d. h. $\lambda_{3}$ ist kleiner und $\lambda_{1}$ größer als der entsprechende wahre Wert, was eine künstliche Zunahme der Anisotropie impliziert und außerdem auch unphysikalische, negativ definite Tensoren zur Folge haben kann. Andererseits wird der Diffusionsabfall bei geringem SNR und entsprechend starker Diffusionswichtung durch den nichtverschwindenden Mittelwert von $I$ in Gleichung (3.13b) nach oben verfälscht. Dadurch unterschätzt man die Beweglichkeit der Wassermoleküle und ermittelt fehlerhafte Werte für den ADC und die Anisotropie, die also in einer entsprechend ungünstigen Konstellation auch zu klein ausfallen kann. Wegen des scheinbar nicht-monoexponentiellen Abfalls des Signals mit dem b-Wert kann die Anwesenheit eines endlichen Rauschuntergrundes irrtümlicherweise zur Interpretation der Meßdaten im Sinne von beschränkter Diffusion oder der Existenz mehrerer Kompartimente führen [50]. Verschiedene Korrekturmechanismen sind bereits in der Literatur (siehe $[48,50]$ und dortige Referenzen) diskutiert aber in dieser Arbeit nicht untersucht oder angewendet worden. Je nach Wahl der Richtungen der kodierenden Gradienten wirkt sich das Rauschen außerdem orientierungsabhängig von deren Anordnung relativ zu den zu messenden Ellipsoiden mehr oder weniger anisotrop aus [51]. Für die Faserverfolgung ist zu berücksichtigen, daß auch die Eigenvektoren durch die Störung des 
Diffusionstensors von ihrer wahren Richtung abweichen und statistisch um diese ideale Achse verteilt sind. Um die Beeinträchtigung durch das Rauschen abzumildern, ist es deshalb oft notwendig, die Rohdaten vor der Auswertung räumlich zu glätten, im einfachsten Fall durch Faltung mit einem Gauß-Kern mit endlichem Träger. Weil auf diese Weise zusätzliche Partialvolumeneffekte eingeführt werden, sollte der Einsatz von kantenerhaltenden Filtern [52] erwogen werden. Auf jeden Fall muß man einen Kompromiß zwischen Rauschunterdrückung und Auflösungsverschlechterung finden.

\subsubsection{Wahl der Parameter für in-vivo Messungen}

Die sinnvolle Konzeption eines Diffusionsexperimentes im Hinblick auf die Meßparameter ist ein diffiziles, weil durch viele hardware- und sequenzspezifische, sowie physiologische Randbedingungen eingeschränktes Optimierungsproblem.

Für in-vivo Messungen und vor allem Anwendungen im klinischen Umfeld ist zu fordern, daß die Datenakquisition insgesamt und wegen Bewegungsartefakten auch für jedes Einzelbild möglichst schnell erfolgt. Außerdem möchte man selbstverständlich eine hohe Auflösung erzielen. Allerdings dürfen die auftretenden Wirbelströme und die Energiedeposition im Probanden weder eine merkliche Nervenstimulation noch einen übermäßigen Temperaturanstieg im Gewebe zur Folge haben. Deswegen unterliegen sowohl Geschwindigkeit und Amplitude der Gradientenschaltung als auch die Anzahl, Stärke und Repetitionsfrequenz der Anregungspulse einer natürlichen, harten Beschränkung. Wie im vorigen Abschnitt diskutiert, müssen die Daten ein minimales SNR aufweisen, damit man Diffusionstensoren zuverlässig berechnen kann, was sich in eine untere Grenze für die erzielbaren Voxeldimensionen übersetzt. Die zuweilen aus diesem Grund verwendeten Bildelemente mit ungleichen Kantenlängen sind abzulehnen, weil sie bestimmte Richtungen auszeichnen und dadurch speziell auch in der Faserverfolgung zusätzliche systematische Fehler herausfordern.

Als Kompromißlösung der in der Fachliteratur kontrovers diskutierten Frage nach der Optimierung der Diffusionspräparation (siehe $[53,54,55,56]$ und Referenzen für eine Übersicht) hat sich in der Anwendung die Praxis etabliert, bei vorgegebener Meßzeit möglichst viele Daten in unterschiedliche Richtungen bei einem hohen b-Wert von ungefähr $1000 \mathrm{smm}^{-2}$ und zusätzlich eine gewisse Anzahl nicht gewichteter Bilder aufzunehmen. Problematisch gestaltet sich das Unterfangen dadurch, daß die optimalen Parameter, unter anderem auch das Zahlenverhältnis von diffusionsgewichteten zu ungewichteten Bildern, von den zu beobachtenden Diffusionskoeffizienten und der Form des Tensors abhängen und deshalb die vorgeschlagenen Kenngrößen wie z. B. das Diffusions-zu-RauschVerhältnis, das SNR eines ADC-Bildes, für ein ausgedehntes Intervall zu maximieren sind. Bei der Wahl der Richtungen und der Anzahl $N_{\mathrm{G}}$ der diffusionskodierenden Gradienten ist die damit verbundene numerische Konditionierung des Gleichungssystems (3.3), also die Robustheit der Berechnung gegenüber Störungen wie dem Meßrauschen, ein Entscheidungskriterium [57]. Darüberhinaus ist es wünschenswert, daß die Richtungen, wegen der a priori unbekannten Orientierungen der zu beobachtenden Fasern, diese maximale Ungewißheit über das zu untersuchende System reflektieren und möglichst isotrop verteilt sind. Jones et al. [58] verwenden dafür einen Algorithmus, der die den Gradientenvektoren ent- 

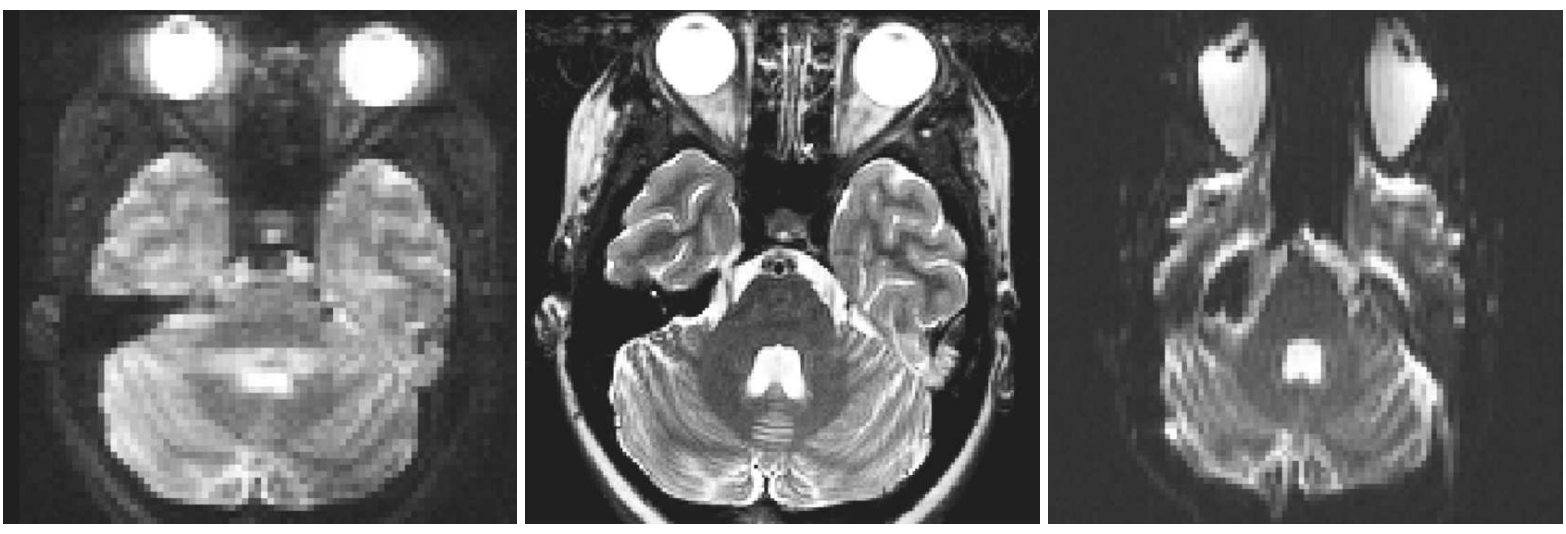

Abbildung 3.4: Anatomiegetreue Abbildung mit DW-SSSTEAM. Dargestellt sind schichtäquivalente, nicht diffusionsgewichtete, transversale Schnittbilder eines menschlichen Gehirns im Hirnstammbereich mit (links) DW-SSSTEAM, (Mitte) einer Anatomie-Sequenz mit $T_{2}$-Wichtung als geometrische Referenz und (rechts) einer Diffusions-EPI-Sequenz.

sprechenden Ortsvektoren abstoßender, punktsymmetrischer Ladungspaare, die auf die Einheitskugel beschränkt sind, durch Energieminimierung ermittelt. Für dieses Schema zeigte Jones [56], daß unter gewissen Voraussetzungen zur robusten, orientierungsunabhängigen Bestimmung des Diffusionstensors mindestens dreißig linear unabhängige Richtungen vonnöten sind. Allerdings ist schon ab ca. zwölf bis fünfzehn Gradientenvektoren eine starke Abnahme der Verbesserung der Meßunsicherheit bei weiter steigendem $N_{\mathrm{G}} \mathrm{zu}$ verzeichnen. Zur Vermeidung der in Abschnitt 3.3.1 angesprochenen Kreuzterme sollte der Diffusionsabfall auf jeden Fall symmetrisch vermessen werden.

Weil orthogonale Gradientensysteme gleichzeitig geschaltet werden können, ist die erzielbare Gradientenstärke entlang der Diagonalen des Scannerkoordinatensystems höher als entlang der Koordinatenachsen. Das führt dazu, daß sich bei der Stejskal-TannerSequenz die minimal erreichbare Echozeit für gegebene Diffusionswichtung mit wachsender Anzahl isotrop verteilter Kodierungsrichtungen hardwarebedingt vergrößert und ein Maximum erreicht, wenn eine davon parallel zu einem Basisvektor orientiert ist. Die wegen des $T_{2}$-Abfalls damit assoziierte SNR-Einbuße muß gegebenenfalls durch eine Anpassung der b-Werte kompensiert oder durch Limitierung von $N_{\mathrm{G}}$ vermieden werden.

Bei der Konzeption des Experimentes sollte man außerdem berücksichtigen, daß vor der erneuten Anregung einer Schicht eine ausreichend großes Zeitspanne abzuwarten ist, um eine hinreichend vollständige $T_{1}$-Relaxation in den Gleichgewichtszustand zu garantieren. Nichtbeachtung dieser Regel bei Diffusionsmessungen führt zu einer systematischen Verfälschung der gemessenen Beweglichkeiten und der Hauptachsen in anisotropen Medien.

\subsection{Die DW-SSSTEAM Sequenz}

Die DW-SSSTEAM-Sequenz (Diffusion-Weighted Single-Shot Stimulated Echo Acquisition Mode), auch als diffusionsgewichteter Turbo-STEAM bekannt, kombiniert die Technik 
der Bildgebung mit stimulierten Echos, die erstmals von Frahm et al. [59] propagiert wurde, mit der Diffusionspräparation nach Stejskal und Tanner und geht auf die Publikationen von Nolte et al. [60, 61] zurück. Da sie allein auf RF-refokussierten Echos beruht, ist diese Aufnahmemethode immun gegenüber Resonanzfrequenzalterationen durch Suszeptibilitätsgradienten, Inhomogenitäten von $\boldsymbol{B}_{0}$ und den damit verbundenen geometrischen Verzerrungen und Intensitätsartefakten. Zur Illustration wird in Abbildung 3.4 eine nicht diffusionsgewichtete DW-SSSTEAM-Aufnahme den äquivalenten Daten einer DiffusionsEPI-Sequenz, die die Grundlage der meisten heutigen Diffusionsbildgebungsexperimente bildet, gegenübergestellt.

Als sogenannter ,Single-Shot“-Sequenz dient DW-SSSTEAM die transversale Magnetisierung einer einzigen Anregung zur Aufnahme des k-Raums des angeregten Volumens (hier: eine Schicht), wodurch die bei „Multi-Shot"-Sequenzen auftretenden Phasenfehler in Form von Unstetigkeiten zwischen den entsprechenden Teilen des Fourier-Raums vermieden werden, die ansonsten aus Bewegungen bei der Messung resultieren können. Gegenüber konkurrierenden Sequenz-Typen [62, 63, 64, 65, 66, 67, 68, 69, 70], die auf Gradienten- oder Hahn-Echo beruhen, muß man nach Abschnitt 2.2.2 mit der Hälfte der ihnen verfügbaren Magnetisierung auskommen, die sich außerdem noch auf alle Phasenkodierlinien verteilt. Das bedeutet einen herben SNR-Verlust.

Im Vergleich vorteilhaft ist dagegen das Verhalten bei einer Feldstärkenerhöhung von $\boldsymbol{B}_{0}$, die prinzipiell in einem besseren Signal-zu-Rausch-Verhältnis resultiert (siehe [71, Abschnitt 4.2]), aber auch meistens mit einer Verringerung von $T_{2}$, einer stärkeren Ausprägung von Suszeptibilitätsgradienten und den damit assoziierten Problemen einhergeht. STE-basierte Sequenzen profitieren allerdings von einem typischerweise verlängerten $T_{1}$, so daß die durch die ersten beiden $90^{\circ}$-Pulse präparierte Magnetisierung entlang der DW-SSSTEAM-Signalpfade länger phasenkohärent gespeichert werden kann.

Nachteilig wirkt sich aus, daß die gewählte Sequenz in Reinform, gemessen an in-vivoVerhältnissen oder gar klinischen Anforderungen, recht langsam ist. Das macht sich z. B. in Abbildung 3.4 durch ein Intensitätsartefakt bemerkbar (horizontaler, weißer Streifen), das wahrscheinlich auf Hirnpulsationen während der Schichtaufnahme zurückzuführen ist. Allgemein steigt mit wachsender Akquisitionsdauer pro Bild natürlich die Gefahr von Bildfehlern durch Bewegung.

\subsubsection{Puls-Schema}

Das Grundprinzip der Sequenz wird im vereinfachenden, nicht maßstabsgetreuen Pulsdiagramm aus Abbildung 3.5 deutlich. Nach der schichtselektiven Diffusionspräparation, die sich aus dem ersten $90^{\circ}$-Puls $\left(90^{\circ}(1)\right)$, dem $180^{\circ}$-Refokussierer und den um ihn gruppierten Diffusionsgradienten (GD) sowie den zugehörigen, unbezeichneten Schichtselektionsund Schichtrephasierungsgradienten (siehe Abschnitt 2.4.1) zusammensetzt, verwendet man das zum Zeitpunkt $T E_{\mathrm{SE}}$ entstehende, diffusionsgewichtete Spinecho (SE) als Eingangssignal für den anschließenden Bildgebungsteil. Die relative Stärke von GD in Schicht- , Phasenkodier- und Frequenzkodierrichtung bestimmt die räumliche Achse entlang welcher der Diffusionskoeffizient gemessen wird. Die zweite bei $t=T E_{\mathrm{SE}}+T E_{\mathrm{STE}} / 2$ applizierte $90^{\circ}$-Anregung $\left(90^{\circ}(2)\right)$ bewirkt eine phasenkohärente Speicherung eines Teils 


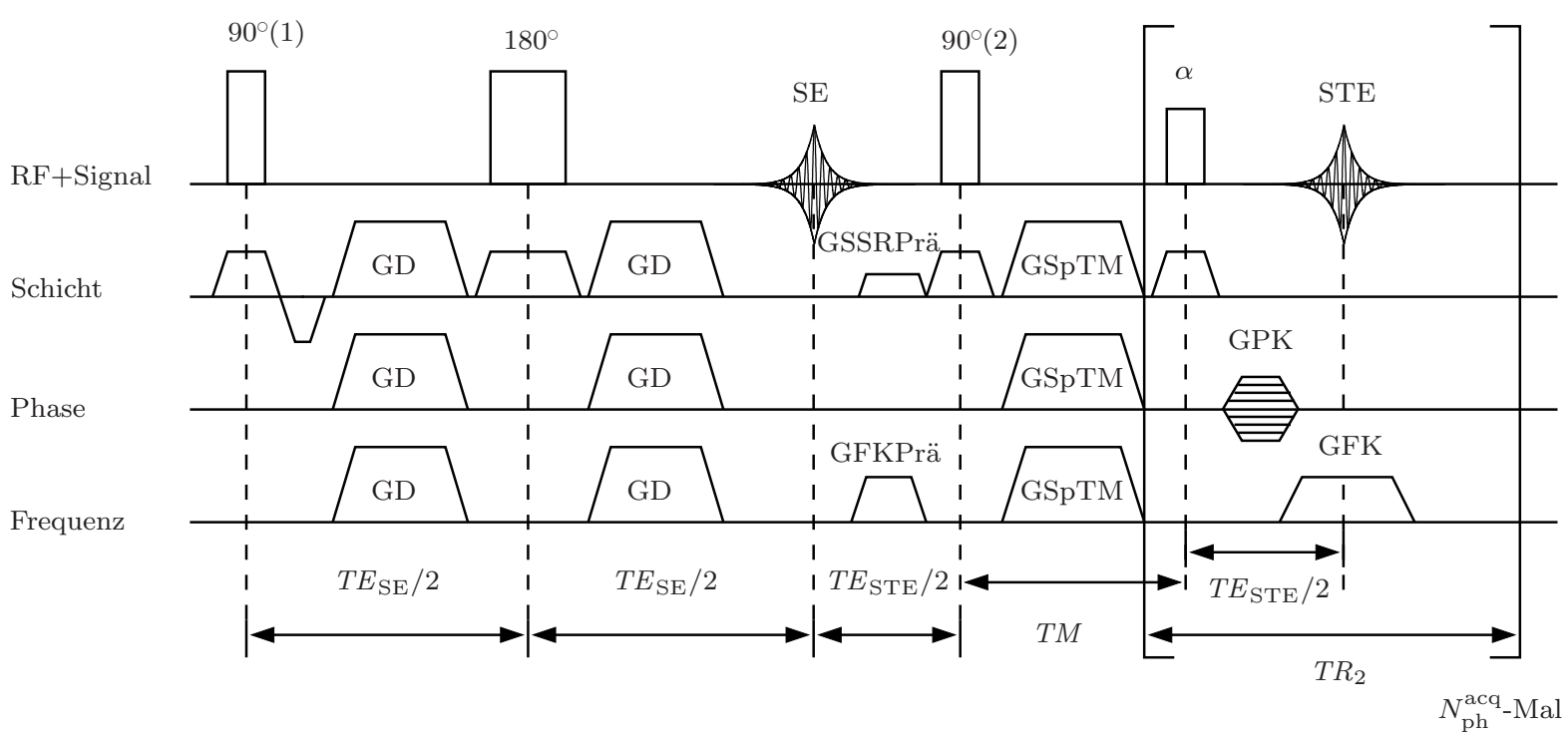

Abbildung 3.5: DW-SSSTEAM. Es sind nur die für die DW-SSSTEAM-Signalpfade relevanten Echos und nicht alle Spoiler eingezeichnet; außerdem ist die Abbildung zwecks besserer Übersichtlichkeit nicht maßstabsgetreu gehalten. Der geklammerte Abschnitt wird nach der Präparationsphase für $N_{\mathrm{ph}}^{\mathrm{acq}}$ der $N_{\mathrm{ph}}$ k-Raum-Linien einer Schicht wiederholt. Die Funktionen der einzelnen Gradienten und RF-Pulse sind im Text näher erläutert.

der präparierten Magnetisierung in Longitudinalrichtung (siehe Abschnitt 2.2.2). Weil die sich nach einem Intervall der Länge TM anschließende Folge von $\alpha$-RF-Pulsen davon in jedem Auslesevorgang nur einen gewissen Anteil verbraucht, kann der k-Raum mit dieser Serie kleinwinkliger Anregungen, die sukzessive stimulierte Echos (STE) von SE erzeugen und jeweils einer k-Raum-Linie entsprechen, mit Hilfe angemessener Frequenz- und Phasenkodiergradienten (GFK, GPK) abgetastet werden. Insgesamt nimmt man nach dem initialen $90^{\circ}$-Puls $N_{\mathrm{ph}}^{\text {acq }}$ Linien jeweils in der Repetitionszeit $T R_{2}$ auf. Die Akquisition einer Schicht erfolgt per definitionem innerhalb eines Intervalls der Länge $T R_{1}$.

Bei den Gradienten GFKPrä und GSSRPrä handelt es sich um sogenannte Prädephasierer. Der zum Frequenzkodierer GFK zugehörige GFKPrä sorgt, wie in 2.4.2 erläutert, für die symmetrische Abtastung des k-Raums in $k_{x}$-Richtung. Allerdings ist er im Gegensatz zur dortigen Darstellung zeitlich durch zwei RF-Pulse vom eigentlichen Lesegradienten getrennt. Durch das Vorziehen in den Präparationsteil verkürzt sich die Meßzeit, weil dieser Abschnitt im Gegensatz zum Auslesesegment während der Schichtaufnahme nur einmal durchlaufen wird. Es ist zu beachten, daß das Vorzeichen dieses Prädephasierers wegen der RF-Echo-bedingten Phaseninversion im STE-Kohärenzpfad dem des Frequenzkodierers entsprechen muß. Auch die Momente der Schichtrephasierer der zweiten 90 Anregung und der $\alpha$-Auslesepulse lassen sich in einem einzigen Gradienten (GSSRPrä) zusammenfassen und vorwegnehmen. Weil die entsprechenden Schichtselektionsgradienten respektive vor und nach dem TM-Intervall in Schichtrichtung dephasierend wirken, muß das zugehörige Moment im ersten Fall das entgegengesetzte und im zweiten Fall das gleiche Vorzeichen wie der auszugleichende Gradient haben. Vom Vorzeichen dieser Summe hängt die Polarität von GSSRPrä ab. Der schichtselektive $180^{\circ}$-Refokussierer ist 
im übrigen selbstrephasierend [71, Abschnitt 3.2].

Um die störenden FIDs, die von den $\alpha$-Auslesepulsen erzeugt werden, während der Datenaufnahme zu unterdrücken, appliziert man direkt nach den entsprechenden Anregungen in Frequenz- und Schichtrichtung zusätzliche Gradienten, die schon erwähnten „Spoiler", die den ungewollten Anteil der Transversalmagnetisierung dephasieren. Damit die Phasenkohärenz des erwünschten STE-Signalpfades zum Zeitpunkt des Auftretens der stimulierten Echos gewahrt bleibt, müssen die Spoiler-Momente, also die Zeit-Integrale der entsprechenden Gradientenfunktionen, zusätzlich von den prädephasierenden Gradienten GFKPrä und GSSRPrä aufgebracht werden. Zusammen bilden diese symmetrischen Gradientenpaare dann jeweils einen „Crusher“, ein Ausdruck für ein Verfahren, mit dem selektiv einige Magnetisierungsbeiträge zerstört und andere bewahrt werden. Im Gegensatz dazu versucht man mit einem reinen Spoiler zumeist unspezifisch die gesamte Phasenkohärenz zu zerstören. Solche Sequenzelemente sind zum Beispiel nach der Vermessung jeder k-Raum-Linie unerläßlich, damit sich keine Echos höherer Ordnung während folgender Datenaufnahmeperioden bilden. Denselben Zweck erfüllen die Gradienten GSpTM während des Mittenintervalls TM.

Ein weiteres Crusher-Paar wird um den Refokussierer angeordnet, da sein Flipwinkel meist nicht über die ganze Probe verteilt genau $180^{\circ}$ beträgt, und somit weitere, störende Transversal- und Longitudinalmagnetisierungsbeiträge entstehen, deren Refokussierung bei Nichtbeachtung zu Streifenmustern im Bild führt. Die Verwendung von CrusherGradienten ist nicht unproblematisch, weil sie ebenfalls eine diffusionssensibilisierende Wirkung haben. Während die Spoiler-Zwillinge um den $180^{\circ}$-Puls einfach in die b-WertBerechnung miteinbezogen werden können, bewirken diejenigen Gradientenpaare, die beidseitig von den durch $90^{\circ}(2)$ - und $\alpha$-Pulsen begrenzten Intervallen angeordnet sind, als Nebeneffekt, daß jede k-Raum-Linie wegen der linear ansteigenden Schaltzeitdifferenzen (und damit der Diffusionszeiten) eine individuelle Wichtung erfährt. Die Amplituden der FID-Spoiler und die TM-Zeit dürfen also nicht zu groß gewählt werden.

Zusätzlich zur gradientenbasierten Zerstörung unerwünschter Echos besteht für diesen Zweck außerdem die Möglichkeit, die Phasen der RF-Pulse zu variieren (RF bzw. Phase Cycling [71, S.67]). Neben Bewegungsartefakten, geringer Geschwindigkeit und dem schwachen Signal stellen solche schädlichen Kohärenzen aufgrund der verschlungenen Signalpfade ein Hauptproblem von DW-SSSTEAM dar. Ein weiterer subtiler Effekt kann dann auftreten, wenn die Longitudinalmagnetisierung während des Ausleseteils der Sequenz auf der dadurch vorgegebenen Zeitskala zu schnell relaxiert. Obwohl man dem FID, der einem $\alpha$-Puls unmittelbar folgt, wie beschrieben, durch einen Spoiler begegnen kann, stellt die Rephasierung dieser Signalkomponenten in den nachgelagerten $T R_{2}$-Intervallen ein Problem dar, insbesondere bei großen Flipwinkeln. Dabei ist es prinzipiell möglich, daß remanente Echos einer Schicht die Aufnahme später gemessener Schnittebenen beeinträchtigen. Das Problem wird durch eine Verkürzung des Auslesesegmentes entschärft, was sich entweder durch eine Erhöhung der Akquisitionsbandbreite oder die im Laufe der Arbeit zu diskutierenden k-Raum-Reduktionstechniken realisieren läßt.

Allgemein ist noch zu bemerken, daß die deutliche zeitliche Trennung von Präparationsund Bildgebungsteil durch das Mittenintervall als Nebeneffekt den Vorteil hat, daß die durch die Diffusionsgradienten potentiell ausgelösten, schnell abklingenden Wirbelströme 
keine bildverzerrende Wirkung haben.

\subsubsection{Die natürliche Punkt-Transfer Funktion: Wahl der Flipwinkel}

Wie in 2.4.4 dargelegt, bestimmt der Fourier-Raum-Abfall durch die Punkt-Transfer Funktion (PSF) die effektive Aufösung eines Bildes. Benutzt man im Ausleseteil der DWSSSTEAM-Sequenz einen konstanten Flipwinkel $\alpha(n)=$ konst. für alle mit $n$ indizierten Anregungen und nimmt die dazu korrespondierenden Linien alternierend-symmetrisch zur k-Raum-Mitte, $k_{y}=0$, auf ( „centric reordering“), d. h. für die mit dem $n$-ten Puls assoziierte Linie gilt

$$
k_{y}(n):=\left\{\begin{array}{cl}
-\frac{n-1}{2} \Delta k_{y} & , n \text { ungerade } \\
\frac{n}{2} \Delta k_{y} & , n \text { gerade }
\end{array}, \quad n=0,1, \ldots, N_{\mathrm{ph}}^{\mathrm{acq}}-1,\right.
$$

so folgt aus den Signalgleichungen

$$
\begin{aligned}
M_{z}(n) & \propto \begin{cases}M_{z}^{0} & , n=0 \\
M_{z}(n-1) \cos (\alpha(n-1)) e^{-T R_{2} / T_{1}} & , n>1\end{cases} \\
M_{\perp}(n) \propto M_{z}(n) \sin (\alpha(n)) &
\end{aligned}
$$

von SSSTEAM für die Amplitude der Transversalmagnetisierung in Abhängigkeit von $k_{y}$ $[61]$

$$
M_{\perp}\left(k_{y}\right) \propto \sin (\alpha) \exp \left[-\left(\frac{T R_{2}}{T_{1}}-\ln (\cos (\alpha))\right) \frac{2}{\Delta k_{y}}\left|k_{y}\right|\right]
$$

unter der Voraussetzung, daß

$$
\left(e^{-T R_{2} / T_{1}} \cos (\alpha)\right)^{-1} \stackrel{!}{\approx} 1
$$

Die Bedingung (3.16b) sorgt unter anderem dafür, daß die k-Raum-Modulation des Signals näherungsweise symmetrisch ist. Aus der Exponentialform $\exp \left[-c\left|k_{y}\right|\right]$ in Gleichung (3.16a) resultiert als natürliche Punkt-Transfer Funktion in $y$-Richtung des Bildes die Lorentz-Kurve

$$
\operatorname{PSF}(y)=\sin (\alpha) \frac{2 c}{c^{2}+y^{2}}
$$

mit der Halbwertsbreite

$$
\mathrm{FWHM}=2 c:=\frac{2 \mathrm{FOV}_{y}}{\pi}\left(\frac{T R_{2}}{T_{1}}-\ln (\cos (\alpha))\right),
$$

(Full Width at Half Maximum) wobei die Beziehung (2.48) angewandt wurde. Damit die Ausdehnung (3.17b) der PSF konsistent mit der Fourier-Auflösung (2.49b) bleibt, muß 


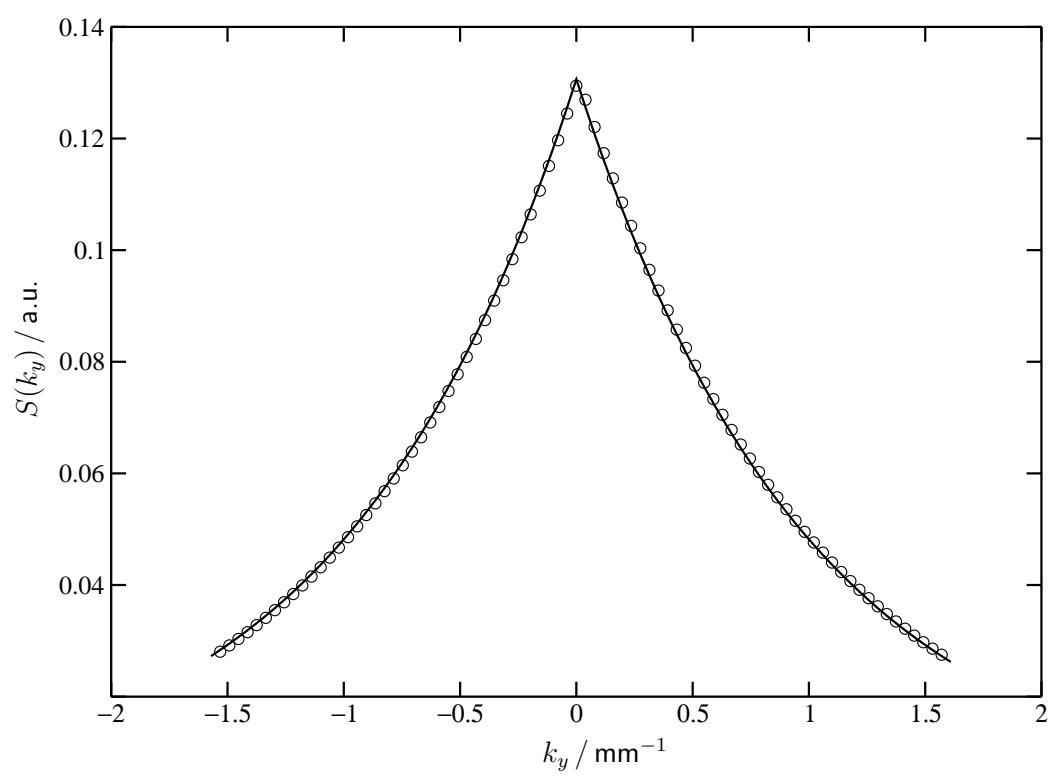

(a) k-Raum-Abfall. Man beachte die leichte Unsymmetrie der Datenpunkte. Bei der sie verbindenden, durchgezogenen Linie handelt es sich um einem Fit an (3.16a).

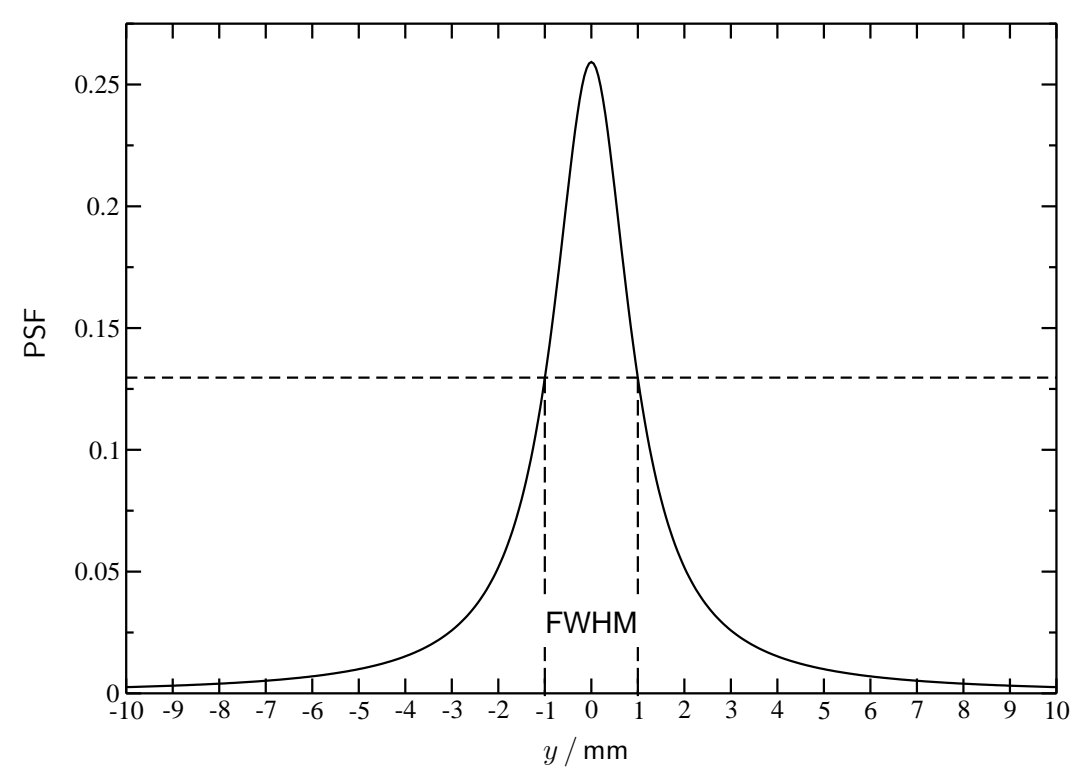

(b) PSF. Dargestellt ist nicht die direkte Fouriertransformierte des kRaum-Abfalls sondern der theoretische Verlauf nach Formel (3.17a).

Abbildung 3.6: Die natürliche Punkt-Transfer Funktion der DW-SSSTEAMSequenz. Simulierte Auswirkungen eines nach Gleichung (3.18) angepaßten, maximalen konstanten Flipwinkels für eine Full Fourier Aufnahme mit Centric Reordering bei einer FourierAuflösung von $\Delta y=2 \mathrm{~mm}$. 

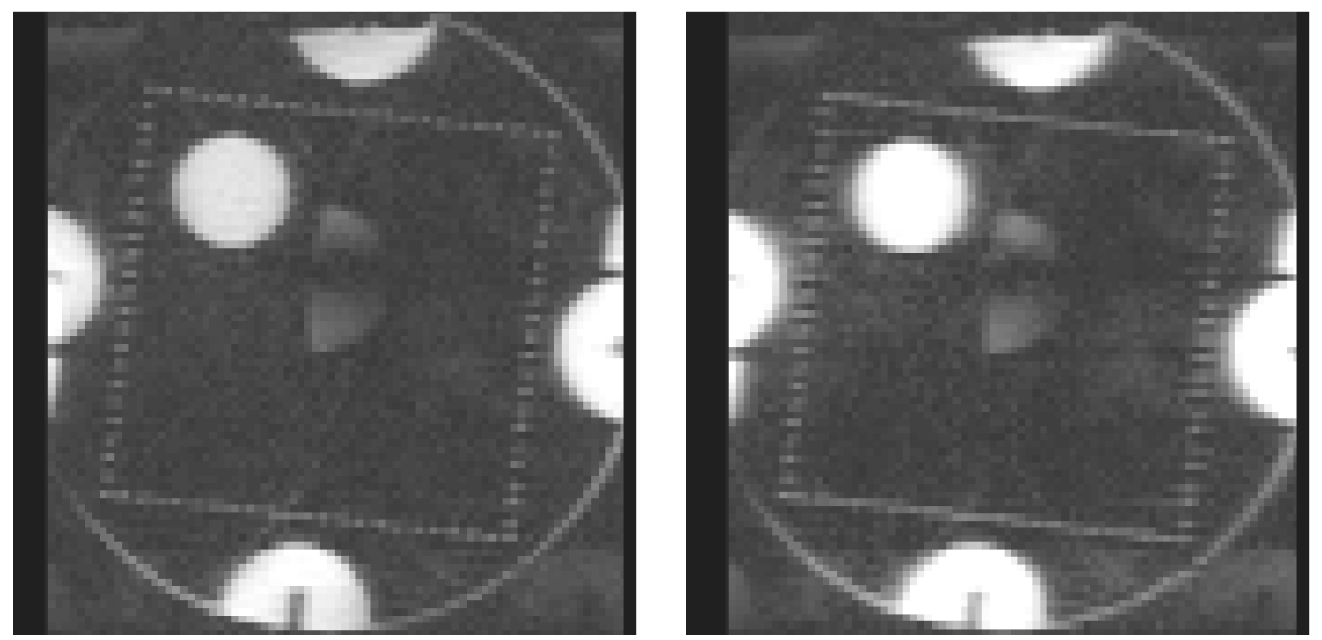

Abbildung 3.7: Breite der PSF und effektive Auflösung. Die Halbwertsbreite der PunktTransfer Funktion in $y$-Richtung (horizontal) entspricht links der einfachen und rechts der doppelten Fourier-Voxel-Abmessung. Die Verletzung der Bedingung (3.18) bewirkt zwar einen Signalanstieg, hat aber auch eine Verschmierung entlang der Phasenkodierrichtung zur Folge.

für die Größe des verwendeten konstanten Auslese-Flipwinkels $\alpha_{\mathrm{FF}}$ die Relation

$$
\alpha_{\mathrm{FF}} \leq \arccos \left(\exp \left[\frac{T R_{2}}{T_{1}}-\frac{\pi}{2} \frac{\mathrm{FWHM}}{\mathrm{FOV}_{y}}\right]\right)
$$

gelten. In Abbildung 3.7 ist der Verschmierungseffekt dargestellt, der auftritt, wenn diese obere Schranke und damit die Aufösungsbedingung FWHM $\leq \Delta y$ verletzt wird.

Die optimale Ausnutzung der zur Verfügung stehenden Magnetisierung ergibt sich durch die Anwendung einer variablen Flipwinkelfunktion, $\alpha(n)$, mit der Randbedingung, daß der letzte Auslesepuls die verbliebene kohärente Longitudinalkomponente vollständig in die Transversalebene klappt [72], also

$$
\alpha\left(N_{\mathrm{ph}}^{\mathrm{acq}}-1\right)=90^{\circ},
$$

wenn man $N_{\mathrm{ph}}^{\text {acq }}$ Echos aufnimmt. Die Anforderung aus Gleichung (3.18) für $\alpha=$ konst. wird dann durch eine iterative Konstruktionsvorschrift für $\alpha_{n} \equiv \alpha(n)$ ersetzt, die sich aus dem Verhältnis aufeinanderfolgender Signalintensitäten $S_{n} \propto\left|M_{\perp}^{\prime}\right|$ herleitet

$$
\frac{S_{n+1}}{S_{n}}=\frac{\sin \left(\alpha_{n+1}\right)}{\tan \left(\alpha_{n}\right)} e^{-T R_{2} / T_{1}},
$$

das dem Quotienten der Funktionswerte der fouriertransformierten PSF an den korrespondierenden k-Raum-Linien entsprechen muß (Abbildung 3.8). 


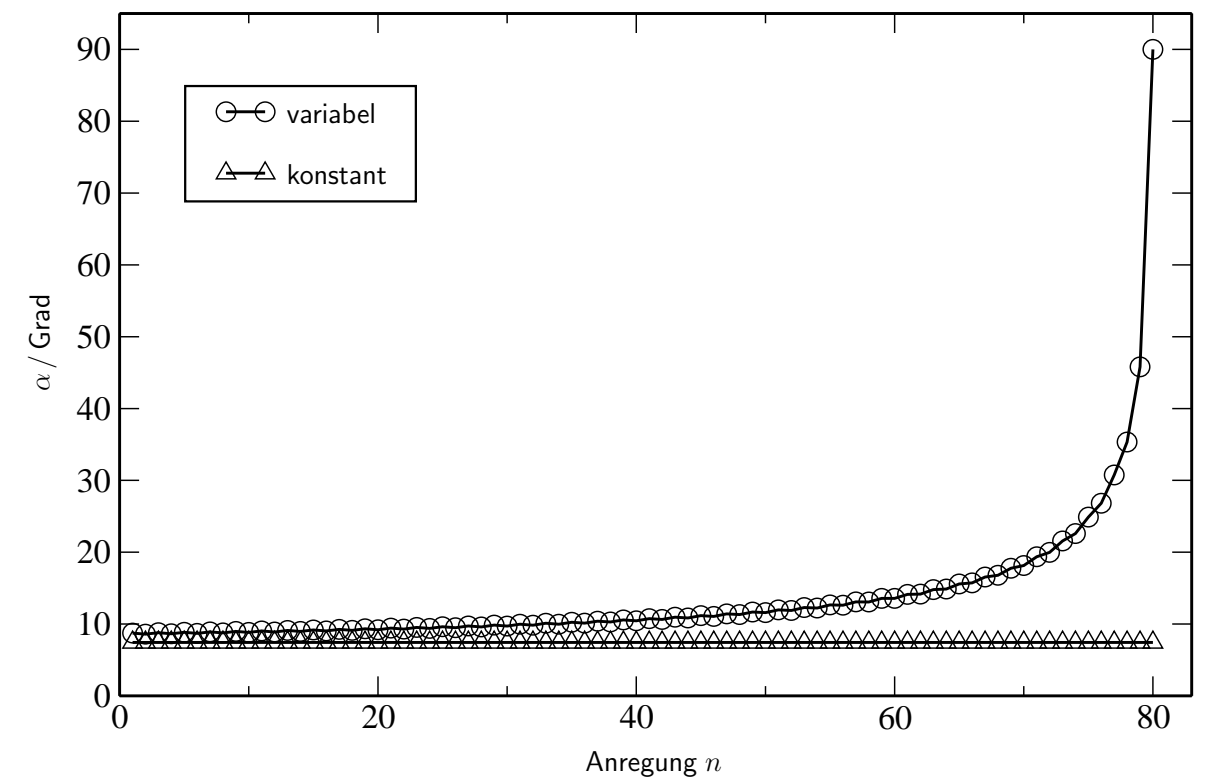

Abbildung 3.8: Flipwinkelschemata. Konstante und variable Flipwinkel für eine lorentzförmige PSF mit FOV $\mathrm{F}_{y}=2 \mathrm{~mm}$ gemäß den Berechnungsvorschriften (3.17a) und (3.20) in Abhängigkeit vom Index $n$ des Anregungspulses.

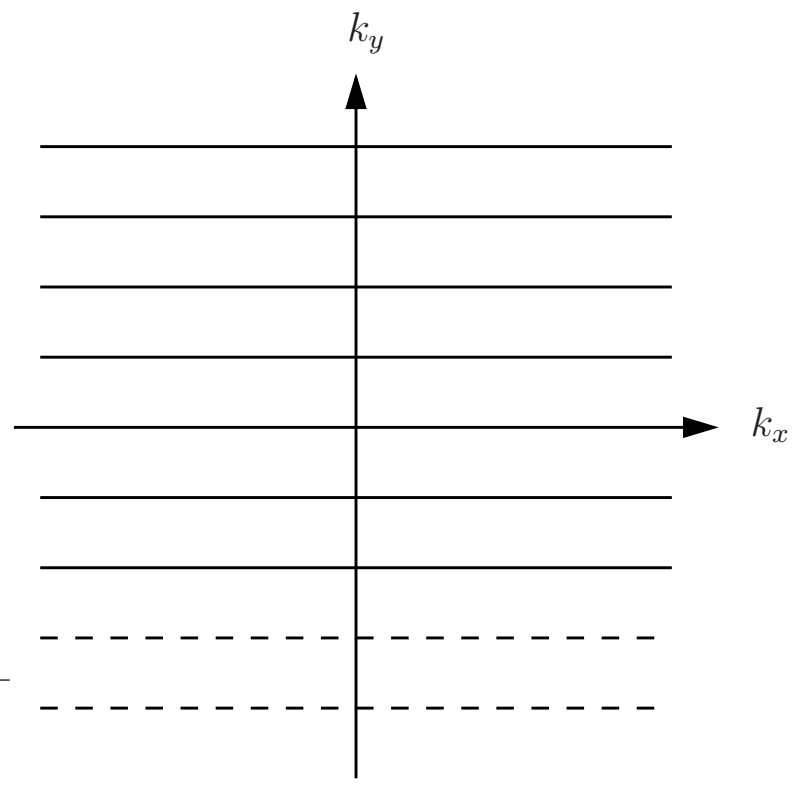

Abbildung 3.9: Partial Fourier. Ausgelassene Linien (gestrichelt) bei der Aufnahme des k-Raums bei Anwendung des Partial Fourier Prinzips. Dargestellt ist eine fiktive 7/9-Messung mit nur zwei gemessenen Phasenkorrekturlinien im negativen $k_{y}$-Bereich. 


\subsection{3 k-Raum-Reduktion I: Partial Fourier}

Weil die Fourier-Darstellung reeller Funktionen $\mathcal{F}^{-1}\{S\}(y)$ eine hermitesche Symmetrie

$$
S\left(-k_{y}\right)=S^{*}\left(k_{y}\right)
$$

zwischen den Fourier-Koeffizienten bei negativen und positiven Frequenzen aufweist, beinhalten die MRI-Meßdaten bei einer vollständigen Abtastung des k-Raums, „full Fourier " (FF), redundante Informationen. Theoretisch ist es also durch Anwendung von Gleichung (3.21) möglich, Bilder aus entsprechend reduzierten Aufnahmen zu rekonstruieren. Diese Methode wird als „partial Fourier"-Technik (PF) bezeichnet. Sie ermöglicht es, die Akquisition zu beschleunigen. Allerdings verschlechtert sich dabei das SNR gemäß (3.12). Weil aber in einer SSSTEAM-Sequenz bei einer verminderten Anzahl von Auslesepulsen mehr Magnetisierung pro Bildlinie zur Verfügung steht, kann diesem Verlust bis zu einem gewissen Grad entgegengewirkt werden; unter vorteilhaften Bedingungen sollte es sogar zu einer Überkompensation kommen [72, Gln.7,8].

Verwendet man variable Flipwinkel in Kombination mit „half Fourier"-Bildgebung $(\mathrm{HF})$, d. h. es werden nur die positiven Frequenzen $k_{y}$ gemessen, lautet die implizite Rekursionsformel für eine lorentzförmige PSF, welche die Bedingung FWHM $\stackrel{!}{=} \Delta y$ erfüllt, wegen $k_{y}(n)=n \Delta k_{y}$ und mit (3.20)

$$
\tan \left(\alpha_{n}\right)=\sin \left(\alpha_{n+1}\right) \exp \left[\pi \frac{\mathrm{FWHM}}{\mathrm{FOV}_{y}}-\frac{T R_{2}}{T_{1}}\right],
$$

woraus sich die benötigten Anregungspulse unter Ausnutzung der Randbedingung (3.19) indexinvertiert berechnen lassen.

Wegen Phasenfehlern, bedingt durch Bewegungen und $\boldsymbol{B}_{0}$-Inhomogenitäten, ist die Fouriertransformierte der Rohdaten einer NMR-Aufnahme oft nicht mehr reell, d. h. dem Bild ist eine unbekannte räumliche Phasenfunktion aufgeprägt. Deshalb führen Rekonstruktionen, die ausschließlich auf Gleichung (3.21) beruhen, zu Artefakten. Eine gängige Technik zur Behebung dieses Problems ist die Akquisition von einigen zusätzlichen niederfrequenten Korrekturlinien, die bei Half Fourier eigentlich ausgelassen werden (Abbildung 3.9). Ein aus den zentralen k-Raum-Daten erstelltes niedrig aufgelöstes Bild wird zur Schätzung der Phasenfunktion, für die man eine langsame Veränderlichkeit voraussetzt, benutzt, um diese dann für eine regularisierte d.h. daten- und phasenkonsistente Rekonstruktion zu verwenden. Für die Beschreibung der verwendeten Methoden sei der Leser auf [17, Kapitel 10] verwiesen.

\subsubsection{Referenzimplementation}

Die Referenzimplementation, der letzte Entwicklungsstand der diffusionsgewichteten Single-Shot STEAM-Bildgebungssequenz, auf der die Weiterentwicklungen in dieser Arbeit aufbauen, ist in den Referenzen [73, 74] dokumentiert. Sie beruht auf der Partial Fourier Technik und nimmt jeweils $N_{\mathrm{ph}}^{\text {acq }}=\frac{5}{8} N_{\mathrm{ph}}$ k-Raum-Linien eines Bildes auf, das dann mit Hilfe des iterativen POCS-Algorithmus (Projection Onto Convex Subsets) [75] 

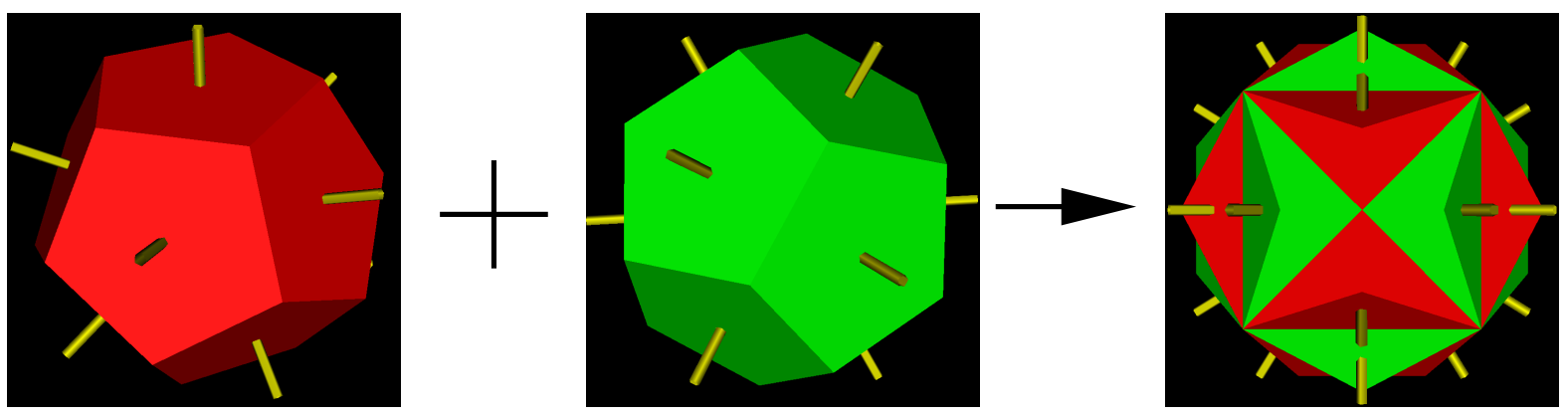

Abbildung 3.10: Standard-Diffusionsrichtungen. Die Gradienten werden in Richtung der Flächennormalen zweier gegeneinander verdrehter Dodekaeder geschaltet.

phasenkorrigiert rekonstruiert wird.

Das Pulsprogramm berechnet standardmäßig variable Flipwinkel für eine Auflösung von $2 \mathrm{~mm}$, wobei das folgende Vorgehen Anwendung findet: in dem um die k-RaumMitte symmetrischen Bereich (zentraler k-Raum), der auf der einen Seite die Phasenkorrekturlinien umfaßt, erfolgt die Aufnahme wie bei Full Fourier alternierend um $k_{y}=0$. Außerhalb dieser Zone gelten Half Fourier Bedingungen. Dort akquiriert man die Linien in aufsteigender Reihenfolge der Wellenvektoren und die Flipwinkel berechnen sich nach der (exakten) Formel (3.22). Für den zentralen k-Raum wird der erste Summand des Exponenten zusätzlich mit dem Faktor 0.5 multipliziert. Diese Strategie setzt die Gültigkeit von (3.16b) voraus und ist deshalb nur eine Näherung, weshalb unter ungünstigen Bedingungen stark unsymmetrische Modulationsfunktionen auftreten können.

Zur Vermeidung von Sättigungseffekten durch unsaubere Schichtprofile regt man das dreidimensionale Meßvolumen im sogenannten ,interleaved“-Modus an, d. h. die geraden Schichtzahlen sind zeitlich vor den ungeraden eingeordnet (oder umgekehrt).

Das Diffusionskodierungsschema sieht die Aufnahme eines ungewichteten $\left(b=0 \mathrm{~s} / \mathrm{mm}^{2}\right)$ und vierundzwanzig gewichteter Bilder $\left(b=1000 \mathrm{~s} / \mathrm{mm}^{2}\right)$ vor. Die Gradienten sind dabei entlang der Flächennormalen von zwei zueinander verdrehten Dodekaedern ausgerichtet (siehe Abbildung 3.10 und Anhang A). Diese Anordnung erlaubt einen relativ effizienten, gleichzeitigen Einsatz der drei Gradientenspulen für die verschiedenen Koordinatenachsen des NMR-Gerätes, was bei dem gewählten maximalen b-Wert in einer minimal erreichbaren Echozeit von $T E_{\mathrm{SE}}=50,1 \mathrm{~ms}$ resultiert. Wegen der Symmetrie des Schemas sind nur zwölf Diffusionsrichtungen linear unabhängig.

Mit den standardmäßig verwendeten isotropen Voxeldimensionen von $2 \times 2 \times 2 \mathrm{~mm}^{3}$ und einem FOV von $160 \mathrm{~mm} \times 256 \mathrm{~mm}$, dauert die Aufnahme eines einzelnen Schnittbildes bei einer Bandbreite von $180 \mathrm{~Hz} /$ Pixel ungefähr $466 \mathrm{~ms}$, wogegen man im Full Fourier Modus 700 ms benötigt. Um beim Menschen das Großhirn abzudecken, sind bei der eingestellten Schichtdicke ca. 50 Schichten vonnöten. Für die Faserverfolgung annehmbare Resultate erzielt man bereits bei nur zweifach magnitudengemittelten Bildern mit einer Gesamtaufnahmezeit von 19 : 25 min. Erfahrungswerte zeigen, daß bei Probandenmessungen eine Meßdauer von 15-20 Minuten noch als tolerabel angesehen wird. Es ist dabei anzumerken, daß die Diffusionsmessung wegen der erforderlichen schnellen Schaltzeiten und hohen Gradientenamplituden als Nebeneffekt zu einer beträchtlichen 
Geräuschentwicklung und zu Vibrationen des NMR-Gerätes führt, die zum einen als unangenehm empfunden werden können und zum anderen durch Bewegungsartefakte die maximale Auflösung einschränken.

\subsubsection{Versuchsaufbau}

Als Meßgerät dient ein für die medizinische Diagnostik zugelassener NMR-Scanner der Firma Siemens (Magnetom Trio), der bei einer $\boldsymbol{B}_{0}$-Feldstärke von 2.9 Tesla arbeitet. Die maximale Gradientenamplitude beträgt $40 \mathrm{mT} \cdot \mathrm{m}^{-1}$ bei einer Schaltgeschwindigkeit von $200 \mathrm{mT} \cdot \mathrm{m}^{-1} \cdot \mathrm{ms}^{-1}$. Das mittels einer Ganzkörperspule erzeugte NMR-Signal wird mit einem sogenannten „phased array“ detektiert, eine Multi-Spulen Konstruktion, die hier aus acht zusammengeschalteten Einzelelementen besteht. Diese Bauart erlaubt eine bessere Ausleuchtung des Versuchsobjekts und liefert ein höheres SNR als konventionelle Empfänger. Allerdings ist die Intensität des kombinierten Gesamtbildes mit einer Funktion der lokalen Spulensensitivitäten moduliert. Für die Diffusionsbildgebung stellt diese Eigenheit jedoch kein Problem dar, weil der Effekt für alle Bilder gleich ist und nicht Absolutwerte sondern nur relative Änderungen bei verschiedenen b-Werten für die Berechnung relevant sind.

Siemens stellt für die Sequenzimplementation auf dem Gerät eine Entwicklungsumgebung, IDEA (Integrated Development Environment for Applications), bereit, die TestWerkzeuge und Software-Bibliotheken als abstrahierte Schnittstellen zu Scanner-Hardware und Bildrechnern umfaßt. Der in der Computersprache $\mathrm{C}++$ verfaßte Ansteuerungscode wird dem Meßrechner kompiliert in Form einer „shared library“ übergeben.

Für in-vivo Untersuchungen stellten sich nach eigenem Ermessen gesunde Probanden zur Verfügung. Die anonymisierte Darstellung der Daten in dieser Arbeit erfolgt mit ihrem Einverständnis. 


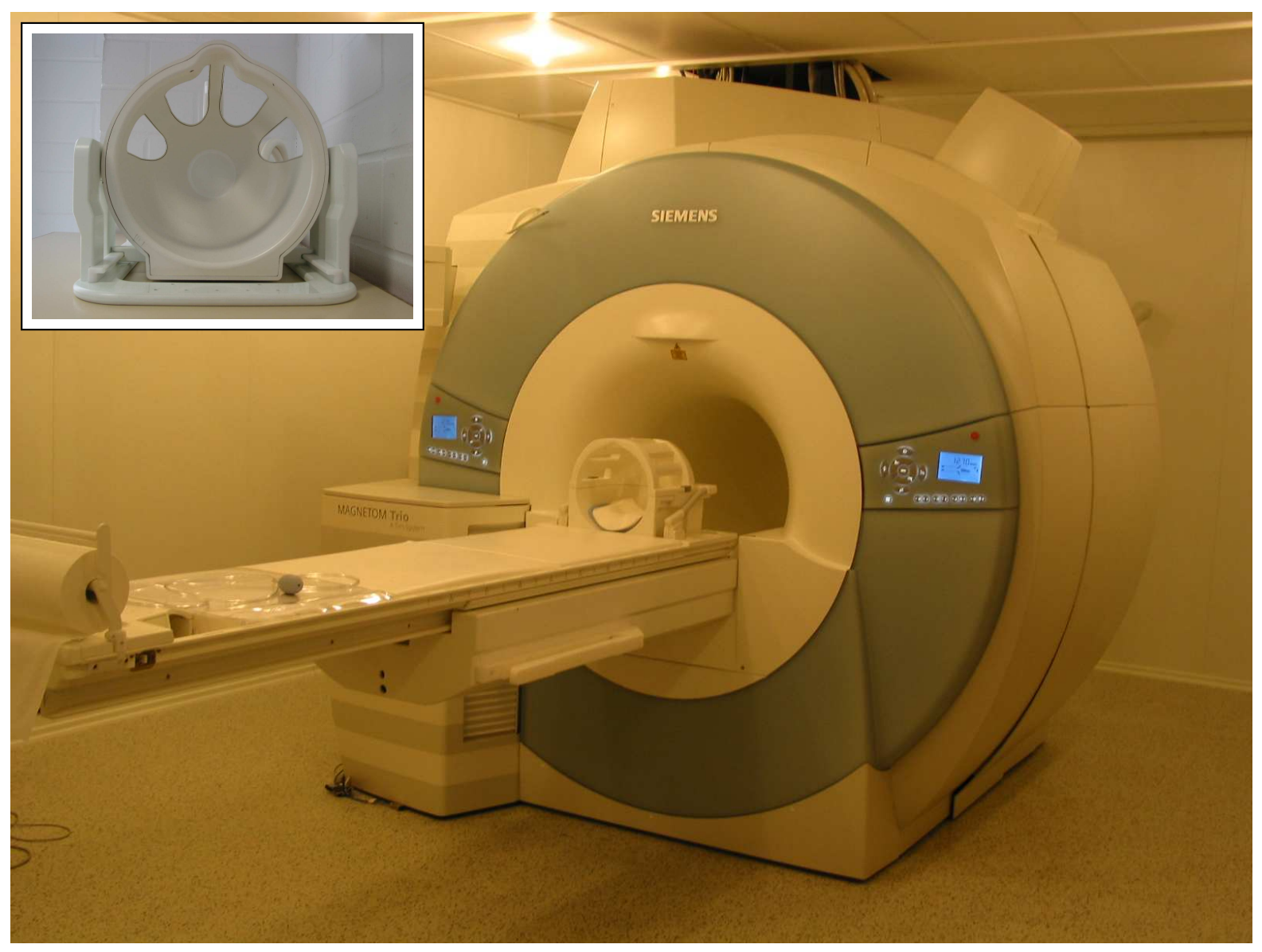

Abbildung 3.11: Versuchsaufbau. NMR-Gerät und Phased Array Aufnahmespule (kleines Bild). 


\section{KAPITEL 4}

\section{Parallele Datenaufnahmetechniken und DW-SSSTEAM}

\section{1 k-Raum-Reduktion II: sensitivitätskodierte Bildgebung}

Neben der auf Symmetriebeziehungen beruhenden k-Raum-Reduktion mit Hilfe der in Abschnitt 3.4.3 erläuterten Partial Fourier Methode hat sich in den letzten Jahren eine weitere Technik zur Beschleunigung der Datenaufnahme etabliert, die auf den ortskodierenden Eigenschaften von Phased Array Spulen fußt. In einer Verallgemeinerung von Gleichung (2.45) ist das Signal im $l$-ten von $L$ Spulenelementen nämlich als Fouriertransformierte der durch seine räumliche Empfangscharakteristik $E_{l}(\boldsymbol{r})$ (Sensitivität) modulierten Transversalmagnetisierung aufzufassen

$$
S_{l}(\boldsymbol{k})=\int \mathrm{d} \boldsymbol{r} M_{\perp}^{\prime}(\boldsymbol{r}) E_{l}(\boldsymbol{r}) e^{\mathrm{i} \boldsymbol{k} \cdot \boldsymbol{r}} .
$$

In konventionellen Messungen fügt man die $L$ Einzelbilder mit der für diese Zwecke optimalen „sum-of-squares“-Methode (SOS) zu einem Gesamtbild zusammen [76, 77].

Wenn die Spulensensitivitätsprofile eines Phased Array Empfängers überlappen und dabei gewisse Voraussetzungen erfüllen, entsteht eine zusätzliche Datenredundanz, die es ermöglicht, Bilder aus einer um den Reduktionsfaktor $R$ ausgedünnten k-Raum-Aufnahme zu rekonstruieren. Während der Datenakquisition wird dann nur jede $R$-te Linie abgetastet (Abbildung 4.1(a)). Weil man bei dieser Methode verschiedene Linien quasi gleichzeitig durch die simultane Verwendung mehrerer Spulen misst, ist das Verfahren unter dem Namen parallele Bildgebung geläufig. Aus Gründen, die im folgenden erläutert werden, erlaubt die Spulengeometrie oft nur eine partielle Aufnahmebeschleunigung, d. h. es ist $R<L$. Deswegen hat sich in der englischen Literatur auch die Abkürzung PPI (Partially Parallel Imaging) eingebürgert. Die Basis für die erfolgreiche Anwendung von PPI legten in unterschiedlichen Formulierungen Sodickson et al. [78] und Pruessmann et al. [79], einige prinzipielle Ideen wurden allerdings schon vorher in der NMR-Literatur 


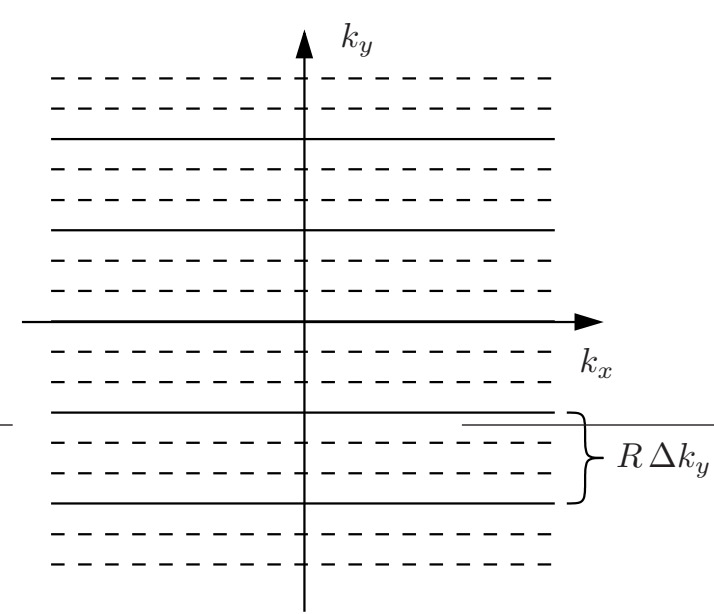

(a) Externe Referenz: das Sensitivitätsprofil der Spulen wird in einer separaten Messung bestimmt.

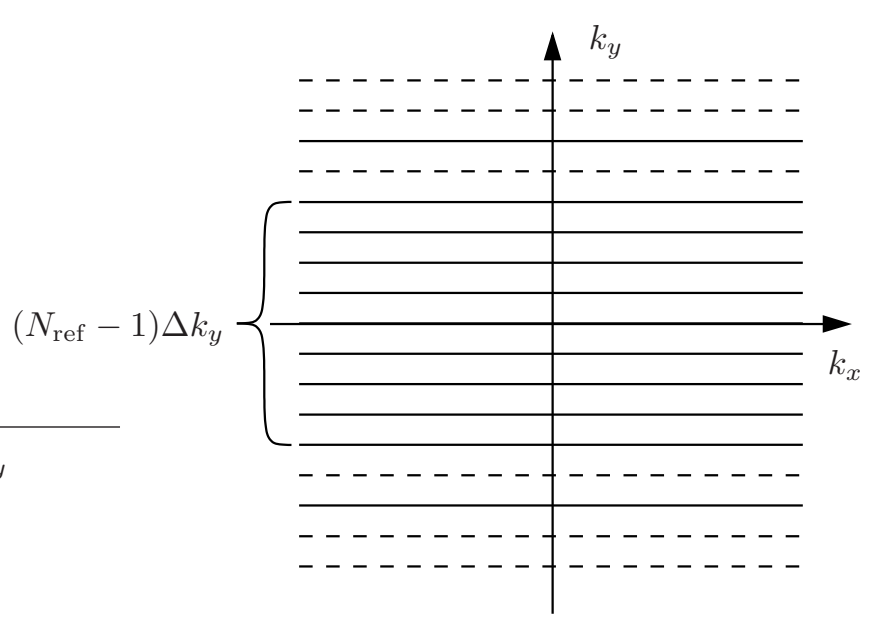

(b) Interne Referenz: die zusätzlich benötigten Spulenkalibrierungslinien werden als Teil der k-Raum-Trajektorie jedes Einzelbildes aufgenommen.

Abbildung 4.1: k-Raum-Abtastung bei paralleler Bildgebung. Die gestrichelten Linien entsprechen den bei $R=3$ ausgelassenen Linien.

diskutiert (siehe z. B. [80] und Referenzen).

Rekonstruktionsalgorithmen für die parallele Bildgebung agieren entweder im Bildoder im k-Raum und werden nach zwei Pionierarbeiten auf diesem Gebiet respektive als SENSE- [79] oder SMASH-artig [78] klassifiziert; es existieren aber auch hybride Ansätze. Desweiteren kommen unterschiedliche Methoden bei der Bestimmung der Sensitivitätsprofile zum Einsatz. Während Verfahren mit externen Referenzlinien (PPI-e, Abbildung 4.1(a)) auf einer separaten Messung beruhen, integrieren Autokalibrierungsverfahren (PPI-i/interne Referenz, Abbildung 4.1(b)) die Gewinnung der für die Rekonstruktion benötigten Spuleninformationen in die Aufnahme der Bilddaten.

Im folgenden beschränken sich die Erläuterungen auf die für diese Arbeit relevante eindimensionale Ausdünnung der k-Raum-Daten in einer Phasenkodierrichtung ( $k_{y}$-Achse), obwohl die parallele Bildgebung in 3D-Sequenzen auf zwei räumliche Dimensionen verallgemeinert werden kann. PPI ist desweiteren nicht zwingend auf die kartesische FourierBildgebung beschränkt.

\subsubsection{SENSE}

Die Verletzung des Abtast-Theorems durch das Auslassen von Phasenkodierschritten hat nach Abschnitt 2.4.4 Aliasing-Effekte zur Folge. Befindet sich bei einer konventionellen, nicht PPI-beschleunigten Messung das Objekt noch gänzlich im Field Of View, $\operatorname{FOV}_{y}(R=$ 1), führt dagegen die oben beschriebene Reduktion der k-Raum-Linien um den Faktor $R$ dazu, daß in den fouriertransformierten Einzelspulendaten ein Bildwert die Summe aus $R$ 

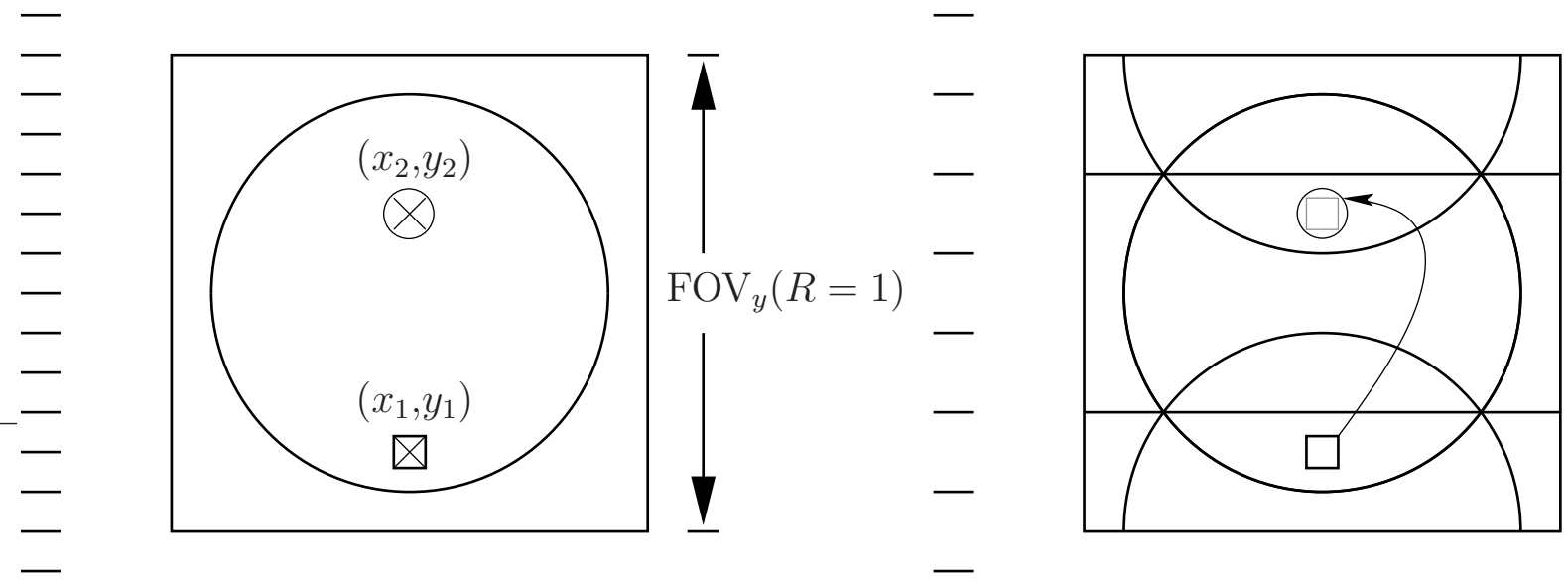

Abbildung 4.2: Aliasing Effekte bei der Verletzung des Abtast-Theorems. (links) Unbeschleunigte Aufnahme. Das kreisförmige Versuchsobjekt befindet sich vollständig in dem durch den Kasten angedeuteten Field Of View. (rechts) PPI-beschleunigte Aufnahme mit $R=2$. Durch die Einfaltungen wird der Voxelwert am Ort $\left(x_{2}, y_{2}\right)$ mit der Bildintensität des um $\operatorname{FOV}_{y}(1) / R$ verschobenen Ortes $\left(x_{1}, y_{1}\right)$ überlagert, also $I_{R=2}\left(x_{2}, y_{2}\right)=I_{R=1}\left(x_{2}, y_{2}\right)+$ $I_{R=1}\left(x_{1}, y_{1}\right)$.

um den $\mathrm{Abstand} \mathrm{FOV}_{y}(1) / R$ entlang der Phasenkodierrichtung verschobenen „wahren “ Bildintensitäten bei $R=1$ darstellt. Dieser Umstand ist schematisch in Abbildung 4.2 skizziert.

Die SENSE-Rekonstruktion (SENSitivity Encoding) [79] ordnet die komplexen Daten der an der Überlagerung beteiligten Bildpunkte von allen Spulen zu einem Vektor $\boldsymbol{a}$ und die Empfängercharakteristiken zu einer $L \times R$ Sensitivitätsmatrix $\mathbf{E}=\left\{E_{l \rho}\right\}$

$$
E_{l \rho} \equiv E_{l}\left(\boldsymbol{r}_{\rho}\right) \quad l=1, \ldots, L ; \quad \rho=1, \ldots R
$$

mit den durch $\rho$ indizierten Ortsvektoren $\boldsymbol{r}_{\rho}$ der in einem Voxel im reduzierten FOV aufeinandergefalteten Objektpositionen. Ist $R$ kleiner als die Anzahl $L$ der verwendeten Aufnahmespulen, so erhält man aus ihren Aliasing-Beziehungen, die in Abbildung 4.2 für ein Einzelbild angedeutet sind, ein überbestimmtes Gleichungssystem in den $R$ unbekannten Pixelintensitäten $I\left(x_{\rho}, y_{\rho}\right)=: v_{\rho}$, das durch Anwendung einer Entfaltungsmatrix $U$ auf den Vektor $\boldsymbol{a}$ analog zu Abschnitt 3.1 im Sinne der Moore-Penrose-Invertierung eindeutig nach den wahren, separierten Bildwerten $\boldsymbol{v} \in \mathbb{R}^{R}$ aufgelöst werden kann

$$
v=\mathbf{U} a
$$

mit

$$
\mathbf{U}:=\left(\mathbf{E}^{*} \boldsymbol{\Psi}^{-1} \mathbf{E}\right)^{-1} \mathbf{E}^{*} \boldsymbol{\Psi}^{-1}
$$

Die Matrix $\boldsymbol{\Psi} \in \mathbb{R}^{L \times L}$ beinhaltet dabei die Korrelationen des Rauschens in der Aufnahmespulenanordnung. Zur vollständigen Bildberechnung muß die beschriebene Entfaltung 
für jeden Bildpunkt innerhalb des reduzierten FOV durchgeführt werden. Die Qualität der Rekonstruktion hängt wesentlich von der ortsabhängigen Kondition des aufzulösenden Gleichungssystems ab, die durch die Form der Sensitivitätsprofile bestimmt wird. Dieser Umstand ist dafür verantwortlich, daß sich das Aufnahmerauschen lokal unterschiedlich in Bildrauschen übersetzt, es gilt also formal

$$
\mathrm{SNR}_{R>1}(\boldsymbol{r})=\frac{\mathrm{SNR}_{R=1}(\boldsymbol{r})}{g(\boldsymbol{r}) \sqrt{R}}
$$

mit dem sogenannten Geometriefaktor $g(\boldsymbol{r}) \geq 1$.

Die Bestimmung der Empfangsprofile ist ein kritisches Element eines SENSE-beschleunigten NMR-Experimentes. Fehler beim Aufstellen der Matrix E äußern sich in periodischen Artefakten in korrelierten Pixeln entlang der beschleunigten Dimension. Die Messung erfolgt gängigerweise über den Vergleich von Bildern der Einzelspulenelemente mit einer Referenzaufnahme durch die in die Röhre des NMR-Tomographen eingelagerte Körperspule, mit welcher bei der Verwendung von Phased Array Spulen gewöhnlicherweise auch der Anregungspuls vermittelt wird. Diese Aufnahmen müssen dabei die Bedingung des Abtast-Theorems für das volle zu messende FOV erfüllen.

\subsubsection{Von SMASH bis GRAPPA}

Im Gegensatz zu SENSE operiert der SMASH-Rekonstruktionsalgorithmus (Simultaneous Acquisition of Spatial Harmonics) [78] im k-Raum. Er beruht auf der Annahme, daß harmonische Funktionen der Ordnung $m$ durch Linearkombinationen der räumlich variablen Spulensensitivitäten $E_{l}(x, y)$ eines Phased Array Empfängers genähert werden können, d. h. man sucht nach Koeffizienten $w_{l}^{(m)}(x)$, so daß

$$
\begin{aligned}
E_{\mathrm{komb}}^{(m)}(x, y) & :=\sum_{l=1}^{L} w_{l}^{(m)}(x) E_{l}(x, y) \\
& \approx e^{\mathrm{i} m \Delta k_{y} y}, \quad m \in \mathbb{N}_{0}
\end{aligned}
$$

mit $\Delta k_{y}=2 \pi / \mathrm{FOV}_{y}(1)$. Dieser Approximationsprozeß wird in Abbildung 4.3 an einem eindimensionalen, synthetischen Beispiel illustriert. Die gewichtete Addition der in $k_{x^{-}}$ Richtung fouriertransformierten Signale $\widetilde{S}_{l}\left(x, k_{y}\right)$ der einzelnen Spulenelemente generiert dann im Idealfall aus den aufgenommenen Linien die um $m \Delta k_{y}$ verschobenen fehlenden Daten

$$
\begin{aligned}
\sum_{l=1}^{L} w_{l}^{(m)}(x) \widetilde{S}_{l}\left(x, k_{y}\right) & =\int \mathrm{d} y \sum_{l=1}^{L} w_{l}^{(m)}(x) E_{l}(x, y) M_{\perp}^{\prime}(x, y) e^{\mathrm{i} k_{y} y} \\
& \approx \int \mathrm{d} y M_{\perp}^{\prime}(x, y) e^{\mathrm{i}\left(k_{y}+m \Delta k_{y}\right) y} \\
& =\widetilde{S}\left(x, k_{y}+m \Delta k_{y}\right)
\end{aligned}
$$




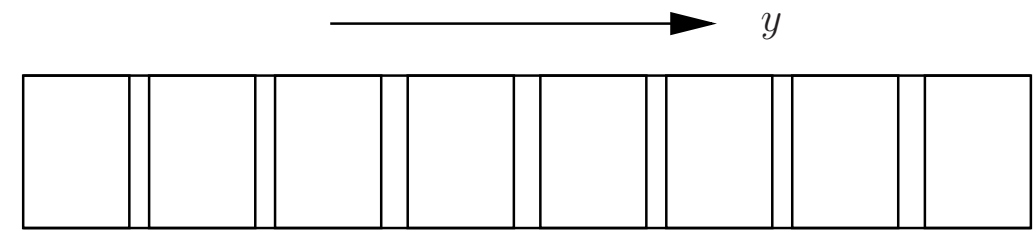

konstant

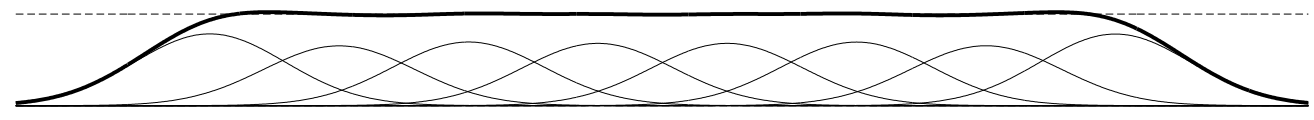

$\cos \left(\Delta k_{y} y\right)$

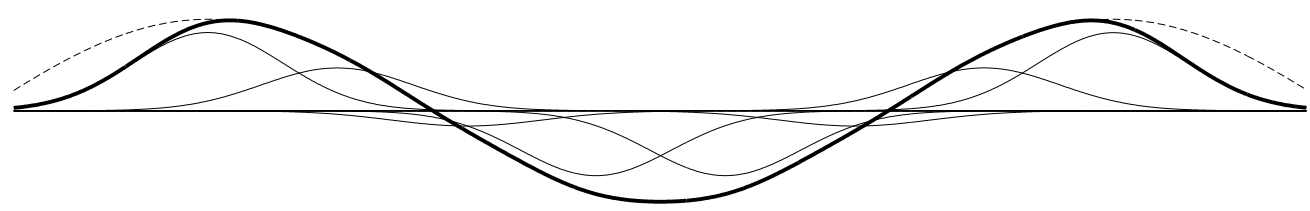

$\sin \left(\Delta k_{y} y\right)$

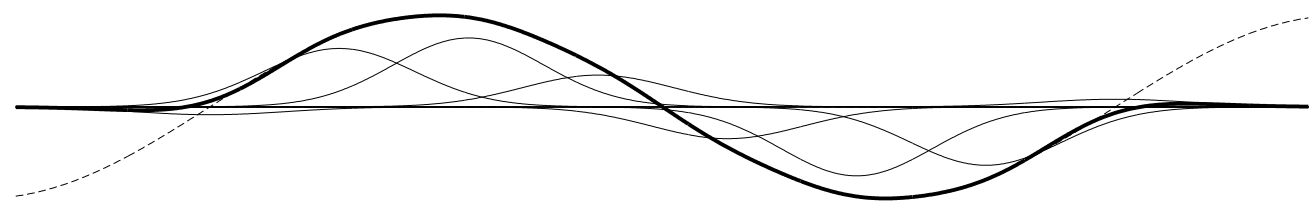

$\cos \left(2 \Delta k_{y} y\right)$

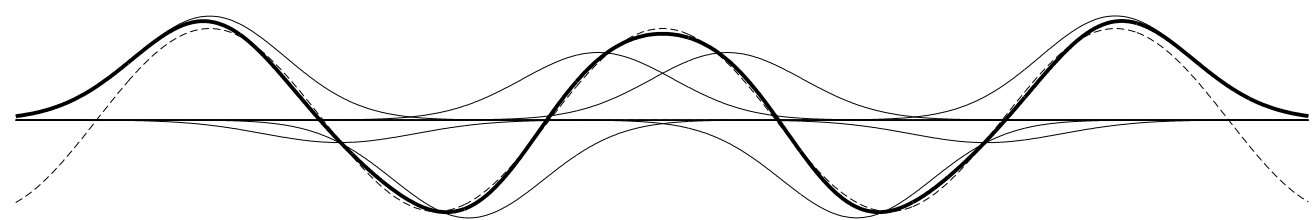

$\sin \left(2 \Delta k_{y} y\right)$

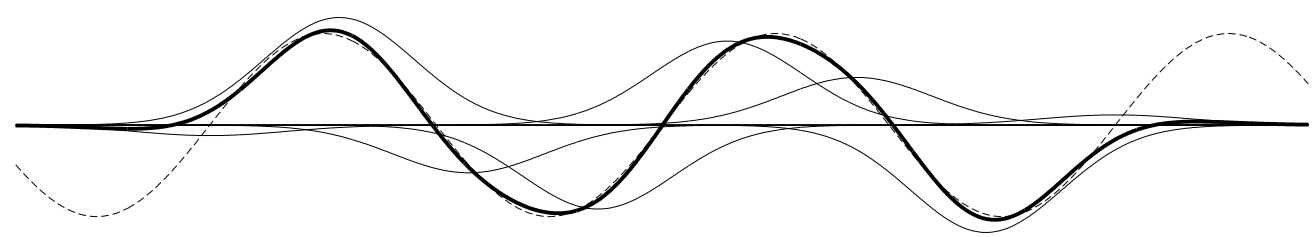

Abbildung 4.3: Prinzip der SMASH-Approximation. Die Empfangsprofile von acht linear entlang der Phasenkodierrichtung $y$ angeordneten Spulenelementen wurden als GaußFunktionen modelliert. Stellt man Gleichung (4.5) für eine vorgegebene Ordnung $m$ an jeder abgetasteten $y$-Koordinate auf, ergibt sich ein (überbestimmtes) lineares Gleichungssystem in den Gewichten $w_{l}^{(m)}$. Die zu nähernden harmonischen Funktionen sind gestrichelt, die Summen der gewichteten Spulenprofile mit dicken Linien gezeichnet (Darstellung in Anlehnung an [78]). 
und entfernt die Modulation durch die Empfangsprofile. Dabei hängt die Rekonstruktionsqualität von der Fähigkeit der Spulenanordnung ab, die benötigten harmonischen Funktionen auf die genannte Art und Weise zu generieren. Diesbezügliche Defizite äußern sich in Geisterartefakten entlang der beschleunigten Dimension. Die Rauschverstärkung durch den g-Faktor bei SENSE findet ihr Analogon bei SMASH in einer oszillatorischen Verschlechterung des SNR durch Rauschkorrelationen zwischen benachbarten kRaum-Linien, die nur dann keine Auswirkungen haben, wenn die Skalarprodukte der als Vektoren aufgefaßten Koeffiziententupel, $\boldsymbol{w}^{(m)}$, für verschiedene Werte von $m$ paarweise verschwinden [81].

Im Gegensatz zu SENSE ist für SMASH-artige Rekonstruktionsmethoden nicht notwendigerweise eine vollständige Sensitivitätsmatrix aufzustellen. Aufgrund der Tatsache, daß die Spulenprofile nur langsam veränderliche Funktionen des Ortes darstellen, kann man deshalb Meßzeit einsparen, indem man die Kalibrierungsmessung und die SMASHApproximation auf der Basis von nur niedrig aufgelösten Referenz-Bildern mit vermindertem $k_{y, \max }$ und entsprechend reduzierter Linienzahl durchführt.

Prinzipielle Probleme bei der in-vivo-Bestimmung der Sensitivitätsinformationen [82] motivierten die Entwicklung von Autokalibrierungsmethoden mit internen Referenzlinien, d. h. die Spulenprofilmessung ist in die Bildaufnahme dadurch integriert, daß der zentrale k-Raum bis zu einer Abschneidefrequenz gemäß der Bedingung des AbtastTheorems und im übrigen mit der reduzierten Rate abgetastet wird (Abbildung 4.1(b)). Die zusätzlich aufgenommenen niederfrequenten Linien tragen außerdem bei Verrechnung mit den PPI-Daten zur Steigerung des SNR und zur Vermeidung von Artefakten bei. Bei dem von Heidemann et al. [83] konzipierten VD-AUTO-SMASH Verfahren (VD steht für „variable density“) werden anders als beim SMASH-Algorithmus die für die Extrapolation benötigten Gewichte $w_{l}^{(m)}$ durch Anpassung der vorhandenen Bilddaten an die Autokalibrierungssignale (AKS) bestimmt

$$
\sum_{l=1}^{L} S_{l}^{\mathrm{AKS}}\left(k_{x}, k_{y}+m \Delta k_{y}\right)=\sum_{l=1}^{L} w_{l}^{(m)} S_{l}\left(k_{x}, k_{y}\right),
$$

d. h. die durch die Phasenkodiergradienten hervorgerufenen, relativen Intensitätsvariationen zwischen zwei Linien und nicht die Spulenprofile, die nur indirekt über die Signalintensitäten eingehen, bilden die Grundlage des Fits. Um die Robustheit der Schätzung der Gewichte gegenüber Rauschen und Gradientennichtlinearitäten zu verbessern, verwendet man für die Approximation (4.7) die Signale sämtlicher paarweiser Linienkombinationen, die einen Abstand von $m \Delta k_{y}$ aufweisen.

Der in dieser Arbeit benutzte GRAPPA-Algorithmus (Generalized Autocalibrated Partially Parallel Acquisitions) geht auf Griswold et al. [84] zurück. In Abwandlung von VD-AUTO-SMASH erfolgt die Rekonstruktion für jede Spule separat und mit einem aufwendigeren Fit-Verfahren. Die Einzelbilder werden dann in einem abschließenden Schritt mit der SOS-Methode zusammengefügt. Weil das recht umständlich darzustellende Vorgehen konzeptionell über die bereits erwähnten Unterschiede hinaus nicht wesentlich von VD-AUTO-SMASH abweicht und außerdem im Detail nicht für das weitere prinzipielle Verständnis der vorliegenden Arbeit wichtig erscheint, sei für die genauen technischen 
Details an dieser Stelle auf die Originalveröffentlichung verwiesen. Gegenüber den vorangegangenen methodischen Entwicklungen verspricht man sich von GRAPPA eine größere Rekonstruktionsstabilität bei für die Erzeugung von harmonischen Funktionen suboptimalen Spulengeometrien bzw. Schichtorientierungen, eine Verbesserung des SNR und die Vermeidung von Intensitätsartefakten aufgrund von unzulänglich phasensynchronisierten Einzelspulen.

Bei der Verwendung des GRAPPA-Algorithmus' ist es prinzipiell möglich, die Aufnahme entweder mit internen Referenzlinien durchzuführen oder aber die für die Rekonstruktion benötigten Spulengewichte aus einer Vorabmessung mit wahlweise reduzierter Aufösung zu gewinnen, um dann die eigentlichen Bilder mit voller Beschleunigung akquirieren zu können (externe Referenz).

\subsubsection{Konsequenzen für DW-SSSTEAM}

Die Anwendung paralleler Aufnahmetechniken auf die DW-SSSTEAM-Sequenz verheißt ähnliche Vorteile wie die in der Referenzimplementation verwendete Partial Fourier Methode. Die Aufnahmezeit pro Bild wird gegenüber einer unbeschleunigten Full Fourier Messung drastisch verkürzt. Dabei wirkt im Hinblick auf die Bildqualität dem sich aufgrund der reduzierte Linienzahl stärker bemerkbar machenden Rauschen der Signalgewinn durch einen höheren Auslesewinkel entgegen. Es sind jedoch Unterschiede im SNRVerhalten der beiden Beschleunigungstechniken zu erwarten, weil zumindest bei paralleler Bildgebung mit externer Referenz und lorentzförmiger PSF in vermehrtem Maß signalintensive zentrale k-Raum-Bereiche eingespart werden. Zu guter Letzt sind die beiden Ansätze zur weiteren Beschleunigung auch miteinander kombinierbar. Allerdings besteht die Gefahr von Artefakten, weil dann ein Teil der für die Partial Fourier Rekonstruktion benötigten Korrekturlinien mit PPI phasenkonsistent geschätzt werden muß.

Generell profitiert die diffusionsgewichtete NMR-Bildgebung am stärksten von den PPI-Verfahren, die auf einer separaten Sensitivitätsmessung beruhen (externe Referenzlinien), weil jede einzelne Schicht vielfach aufgenommen wird, und der zusätzlich benötigte zeitliche Aufwand bei einer großen Zahl von Diffusionsrichtungen zu einer vernachlässigbaren Größe gerät. Dagegen skaliert die Menge der Rekonstruktionsdaten für die Spulenprofile bei Autokalibrierungsmethoden mit der Anzahl der Akquisitionen. Es ist dabei außerdem zu beachten, daß die inhärente SNR-Schwäche der diffusionsgewichteten Bilder eine robuste Bestimmung der Empfangscharakteristik bzw. die Schätzung der Spulengewichte zumindest erschwert und dadurch schwierig zu detektierende Artefakte entstehen können. Auf der anderen Seite verliert unter in-vivo Bedingungen eine den Diffusionstensoraufnahmen vorangeschickte Eichung aufgrund von Bewegungsartefakten an Kongruenz.

Rekonstruktionsfehler durch die Akquisitionsbeschleunigung in Gestalt von periodischen Geisterartefakten sind bei quantitativen Methoden wie DTI natürlich besonders fatal, weil in den berechneten Karten auf subtile Weise die Eigenschaften von räumlich nicht zusammengehörigen Positionen zu einem gewissen Grad miteinander vermischt werden.

Als allgemeines Merkmal PPI-beschleunigter Aufnahmen ist noch die eingeschränkte 
Wahl der Schichtorientierung zu nennen, die aus der damit verbundenen numerischen Konditionierung der Rekonstruktion resultiert. In der Praxis wirkt sich das manchmal in einer suboptimalen Abdeckung des Untersuchungsgebietes aus. Außerdem sind anatomische Atlanten vielfach auf gewisse standardisierte Ansichten festgelegt. Aus diesem Grund ist es in den meisten MRI-Messungen wichtig, isotrope Voxel zu verwenden, um eine sinnvolle Schichtreformatierung mittels Interpolation zu ermöglichen.

\subsection{Verallgemeinerung der Flipwinkelberechnung}

Weil die k-Raum-Trajektorie $k_{y}(n)$ sich bei paralleler Bildgebung durch die Verminderung der Linienzahl ändert, ist bei der Datenaufnahme mit einer auf diese Weise beschleunigten DW-SSSTEAM-Sequenz eine Anpassung der Flipwinkelfunktion $\alpha(n)$ zur Erhaltung der Punkt-Transfer Funktion und der vorgegebenen Auflösung in $y$-Richtung in jedem Fall erforderlich. Für externe Referenzlinien besteht theoretisch die Möglichkeit, einen konstanten Flipwinkel zu verwenden, indem man in Gleichung (3.14) $\Delta k_{y}$ durch $\Delta k_{y} R$ ersetzt, da sich das FOV um den Faktor $1 / R$ reduziert. Bei Full Fourier Bildgebung muß also gelten

$$
\alpha_{\mathrm{FF}} \leq \arccos \left(\exp \left[\frac{T R_{2}}{T_{1}}-\frac{\pi}{2} \frac{\mathrm{FWHM}}{\mathrm{FOV}_{y}} R\right]\right) .
$$

In Abbildung 4.4 sind dazu der numerisch nach (3.15) berechnete k-Raum-Abfall und das maximal wählbare $\alpha_{\mathrm{FF}}$ in Abhängigkeit vom Reduktionsfaktor $R$ aufgetragen.

Für die Sequenzparameter werden im folgenden die Einstellungen aus Abschnitt 3.4.4 vorausgesetzt und der $T_{1}$-Wert des interessierenden Nervenfasergewebes mit $700 \mathrm{~ms}$ bei ca. drei Tesla konservativ nach unten abgeschätzt. In der Literatur zur $T_{1}$-Messung bei dieser Feldstärke (siehe [85] für eine Übersicht) findet man regions- und studienabhängig einen Wertebereich von 700-850 ms für die weiße Substanz, die das eigentliche Zielgewebe für die Untersuchung langreichweitiger neuronaler Konnektivität darstellt. Allerdings durchsetzen viele der betreffenden Axone auch subkortikale Kerngebiete, weshalb es angebracht erscheint, auch die Spin-Gitter Relaxationszeit von 990-1760 ms in grauer Substanz zu berücksichtigen. Eine Unterschätzung dieses Wertes bei der Berechnung der Flipwinkelfunktion bedeutet aber höchstens eine schärfere PSF. Außerdem ist in dem Parameterbereich der Messungen nur eine schwache $T_{1}$-Abhängigkeit des maximal erreichbaren Flipwinkels zu verzeichnen.

Die Graphen in den Abbildungen 4.4(a) und 4.4(c) machen deutlich, daß die Symmetrieannahme (3.16b) mit zunehmendem $R$ nicht mehr vorausgesetzt werden kann. Außerdem läßt sich das Konzept eines konstanten Anregungswinkels nicht sinnvoll auf inhomogene Abtastungsschemata des k-Raums, wie sie bei paralleler Bildgebung mit internen Referenzlinien oder bei Partial Fourier Aufnahmen auftreten, übertragen, was Abbildung 4.5 veranschaulicht.

Weil auch die Konstruktion der variablen Flipwinkelfunktion nach Abschnitt 3.4.4 bei symmetrischer k-Raum-Akquisition auf Gleichung (3.16b) beruht, erfährt der Ansatz an dieser Stelle eine einfache Verallgemeinerung, indem jetzt $\alpha(n)$ gemäß einer frei wähl- 


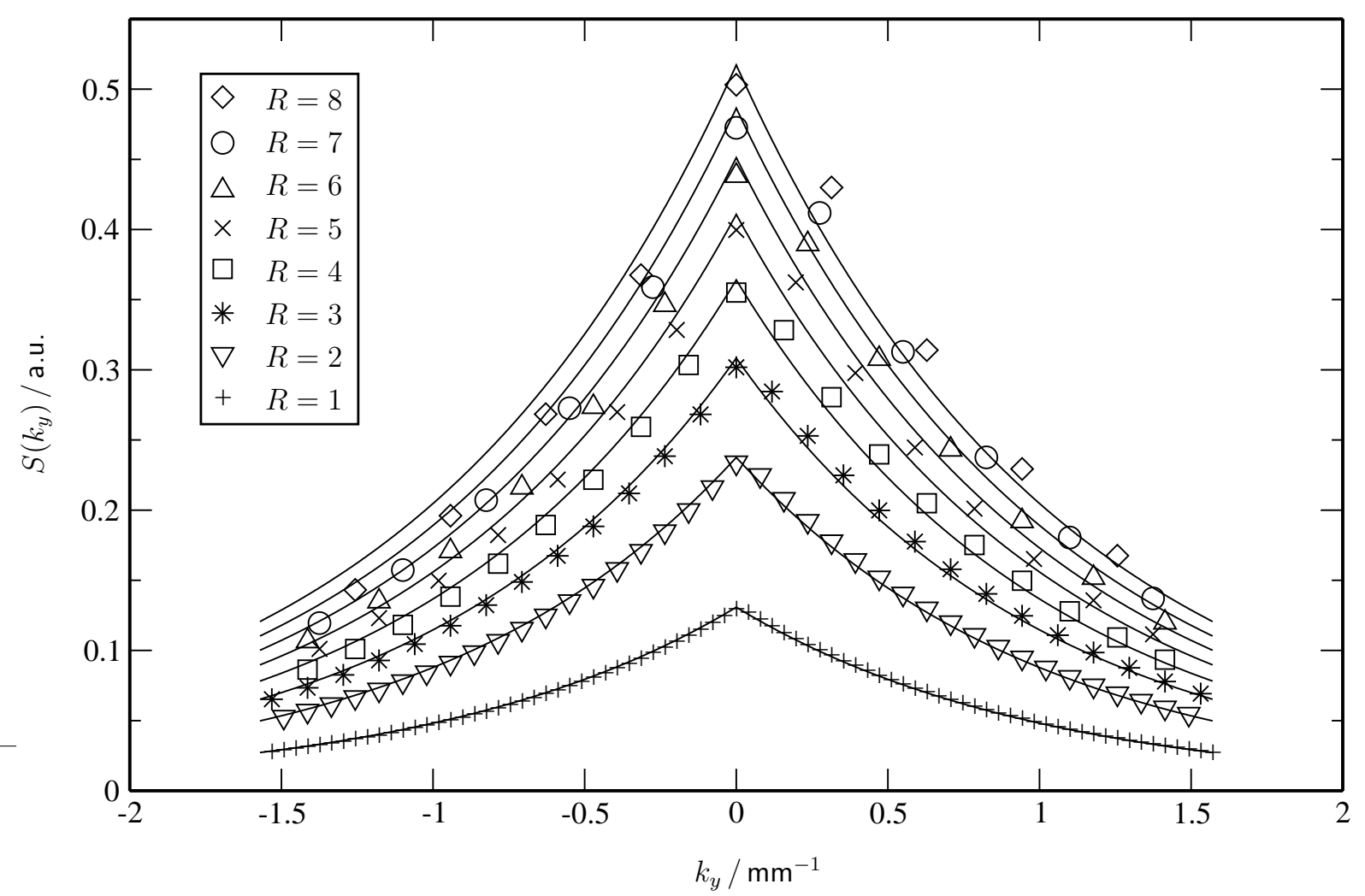

(a) k-Raum-Abfall für verschiedene Reduktionsfaktoren bei optimalem Flipwinkel und angepaßte Fit-Kurven (durchgezogene Linien) der Form $f(k)=a \exp [-b|k|]$.

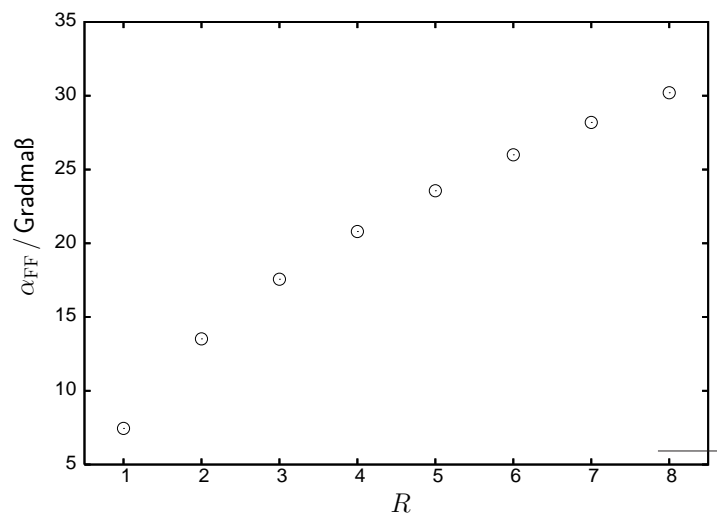

(b) Optimaler Flipwinkel in Abhängigkeit vom Reduktionsfaktor nach Gleichung (4.8).

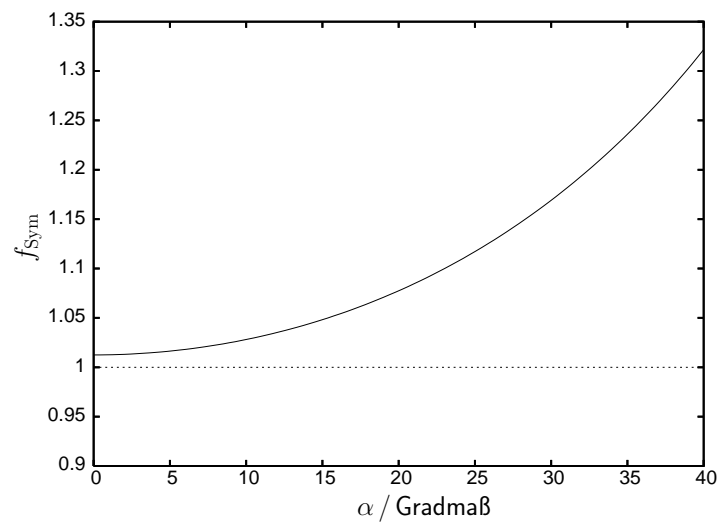

(c) Verletzung der Symmetriebedingung (3.16b) mit steigendem Flipwinkel; Symmetriefaktor $f_{\text {Sym }}:=\left(e^{-T R / T_{1}} \cos (\alpha)\right)^{-1}$.

Abbildung 4.4: DW-SSSTEAM mit PPI und konstantem Flipwinkel. Theoretische Auswirkungen auf den k-Raum-Abfall in Full Fourier Aufnahmen. 
baren, nicht mehr auf die Lorentz-Kurve beschränkten Punkt-Transfer Funktion durch explizite Vorgabe des entsprechend benötigten Funktionswertes an jedem k-Raum-Punkt berechnet wird. Das ist möglich, weil die in longitudinaler Richtung gespeicherte, diffusionspräparierte Magnetisierung in dem durch Gleichung (3.20) eingeschränkten Rahmen beliebig zur Formung des k-Raum-Abfalls und (über dessen Fouriertransformierte) der PSF eingesetzt werden kann. Mit diesem Vorgehen ist auch die Einschränkung auf die alternierend-symmetrische k-Raum-Trajektorie obsolet; sie wird an dieser Stelle der Einfachheit halber und aufgrund ihrer geringen Anfälligkeit gegenüber Bewegungsartefakten trotzdem beibehalten. Allgemein muß gefordert werden, daß die Flipwinkel so gewählt sind, daß für das Signal $S_{n}$ der $n$-ten aufgenommenen Linie gilt

$$
\frac{S_{n+1}}{S_{n}}=\frac{\mathcal{F}\{\mathrm{PSF}\}\left(k_{y}(n+1)\right)}{\mathcal{F}\{\mathrm{PSF}\}\left(k_{y}(n)\right)} .
$$

Diese Gleichung stellt nach der Wahl einer speziellen PSF in Kombination mit der Randbedingung (3.19) wieder eine iterative Konstruktionsvorschrift für die Auslesewinkel dar. Abbildung 4.5 zeigt einen Vergleich verschiedener Flipwinkelfunktionen beim Einsatz der Partial Fourier Technik ohne zusätzliche Beschleunigung durch parallele Bildgebung. Die neuartige Berechnung der Anregungspulse nach (4.9) bringt in diesem Beispiel im Vergleich zur alten Methode bei Lorentz-Kurven nur einen schwachen Signalgewinn (+1,3\%), hat aber den Vorteil der Symmetrie und der Erweiterbarkeit auf beliebige k-RaumTrajektorien. Die Vorgabe einer gaußförmigen Punkt-Transfer Funktion führt zwar zu einem geringeren Verschmierungsgrad in den Bildern, ist aber mit einem deutlich niedrigerem Flipwinkel für die nullte k-Raum-Linie verbunden. Das ist darauf zurückzuführen, daß mehr Magnetisierung für den äußeren Fourier-Raum verwendet wird. Wegen

$$
\mathrm{SNR} \propto \sin \left(\alpha\left(n\left(k_{y}=0\right)\right)\right)
$$

hat man deswegen mit einem reduzierten SNR zu rechnen.

\subsection{Theoretische Auswirkungen}

In den folgenden Abschnitten wird anhand der numerisch optimierten variablen (4.9) oder konstanten (4.8) Anregungspulse der Konfigurationsraum der DW-SSSTEAM-Sequenz untersucht, um die unterschiedlichen Akquisitionsmethoden und Flipwinkelfunktionen zu bewerten und sinnvolle Parameterbereiche herauszuarbeiten. Abbildung 4.6 faßt alle hier betrachteten Kombinationsmöglichkeiten von Punkt-Transfer Funktionen und Datenreduktionstechniken (PF, PPI-e, PPI-i), die durch ihre Signalverteilung im k-Raum charakterisiert sind, zusammen. Soweit nicht explizit anders angegeben, hat die PSF im folgenden eine Lorentz-Form.

Die verwendeten Formeln setzen eine in gewissem Sinn ideale PPI-Rekonstruktion voraus, indem die Rauschzunahme nur über die verringerte Linienzahl eingeht, aber keine zusätzlichen lokalen Verstärkungen, z. B. durch den g-Faktor (4.4), berücksichtigt werden. 


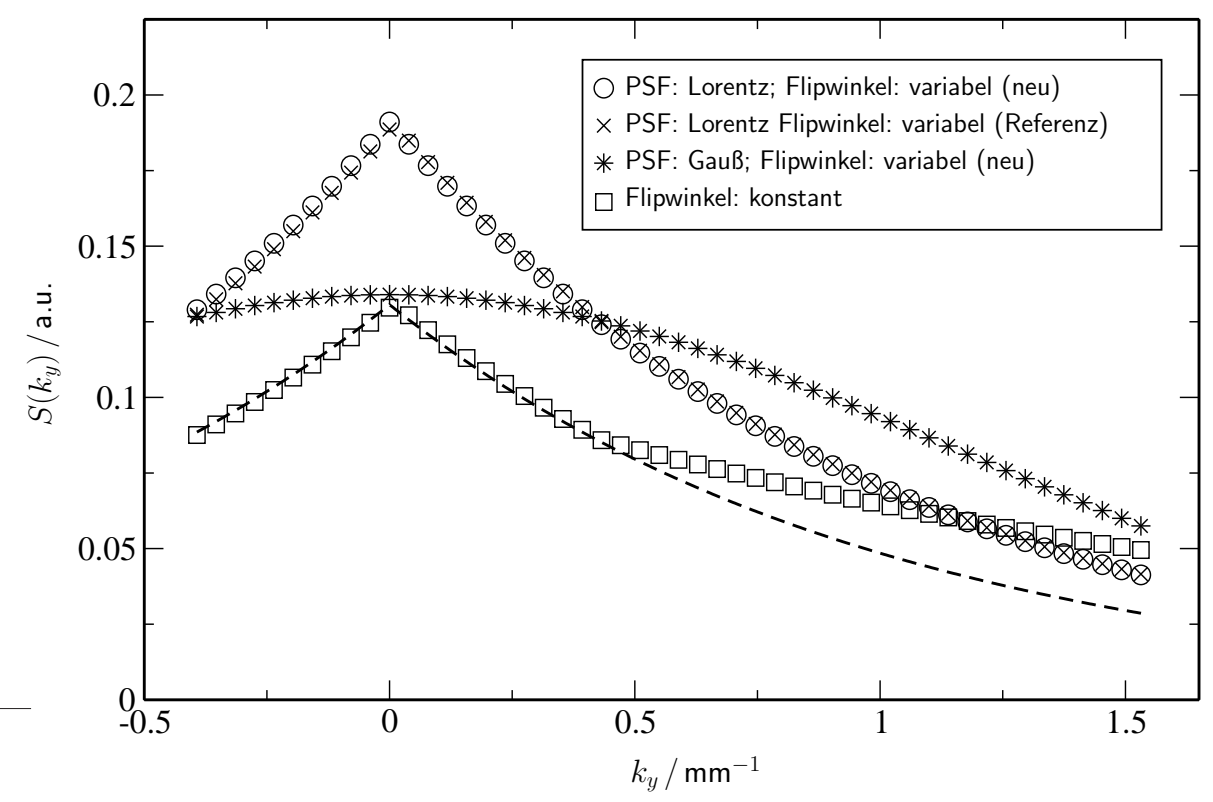

Abbildung 4.5: Notwendigkeit einer verallgemeinerten Flipwinkelberechnung. Die Darstellung vergleicht die theoretische Modulation des k-Raums für verschiedene Flipwinkelfunktionen bei einer 5/8-Partial Fourier Aufnahme. Die Methode der Referenzimplementation aus Kapitel 3.4.4 ist unsymmetrisch. Der Signalabfall für $\alpha(n)=$ konst. induziert wegen der notwendigerweise inhomogenen Abtastung des Fourier-Raums keine lorentzförmige PSF mehr, wie sie durch eine k-Raum-Modulation von der Form der gestrichelten Linie hervorgerufen würde. Nur die verallgemeinerte Flipwinkelberechnung führt zu einem symmetrischen Verlauf und kann auch andere Punkt-Transfer Funktionen wie das Gauß-Profil erzeugen.

\subsubsection{Reduktionsfaktor}

Für eine theoretische Vorhersage über die Auswirkungen von paralleler Bildgebung auf die Datenqualität, wurde das zu erwartende relative SNR in Abhängigkeit vom Reduktionsfaktor $R$, also indirekt von der Anzahl der tatsächlich aufgenommenen Linien $N_{\mathrm{ph}}^{\text {acq }}$ gemäß

$$
\mathrm{SNR} \propto \sin \left(\alpha\left(n\left(k_{y}=0\right)\right)\right) \sqrt{N_{\mathrm{ph}}^{\mathrm{acq}}}
$$

aus dem Flipwinkel für die nullte k-Raum-Linie abgeschätzt.

Wie aus Abbildung 4.7 hervorgeht, ist bei Verwendung eines Autokalibrierungsverfahrens (interne Referenz) das SNR-Verhalten erwartungsgemäß von der Anzahl $N_{\text {ref }}$ der Referenzlinien abhängig. Bei $N_{\text {ref }}=17$ ist bis zu $R=3$ keine wesentliche Verschlechterung oder Verbesserung gegenüber der Full Fourier (FF) Akquisition zu erwarten. Wird eine genauere Kalibrierung benötigt, muß man mit einem kontinuierlichem Qualitätsverlust in den Einzelbildern rechnen. Eine Aufnahme mit 25 Referenzlinien hat für $R=2(\mathrm{FF}+\mathrm{R} 2-\mathrm{i})$ dabei ungefähr das gleiche SNR wie eine 5/8 Partial Fourier Messung (PF 5/8). Dagegen zeigen alle Kurven, die auf der Bestimmung der Sensitivitätsprofile durch eine separate Eichung beruhen, einen vielversprechenden Anstieg des Signal-zu-Rausch-Verhältnisses, 


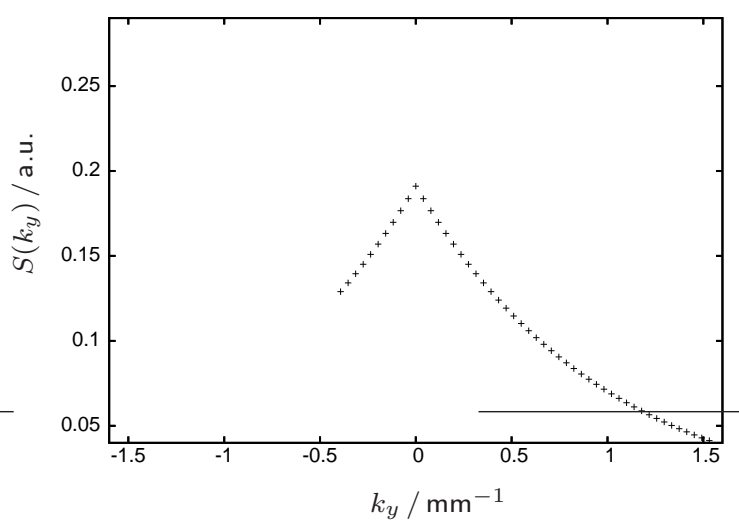

(a) PF, PPI: - $(R=1)$, PSF: Lorentz, $\alpha(n)$ : variabel.

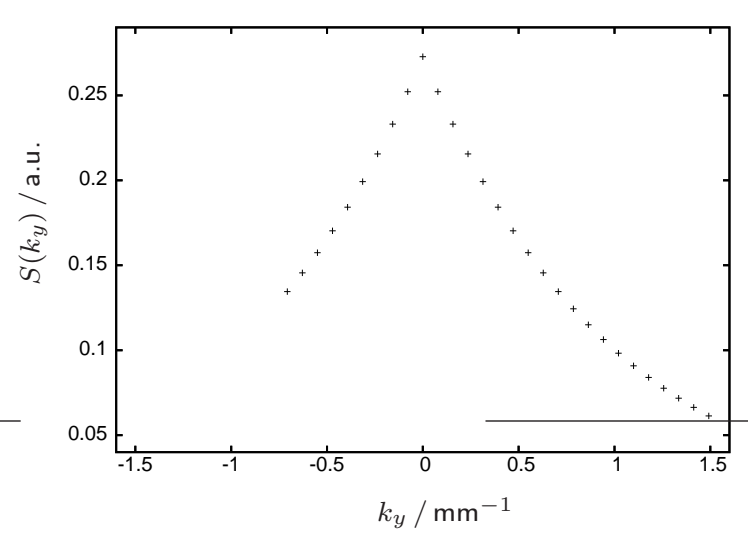

(c) PF, PPI: extern, PSF: Lorentz, $\alpha(n)$ : variabel.

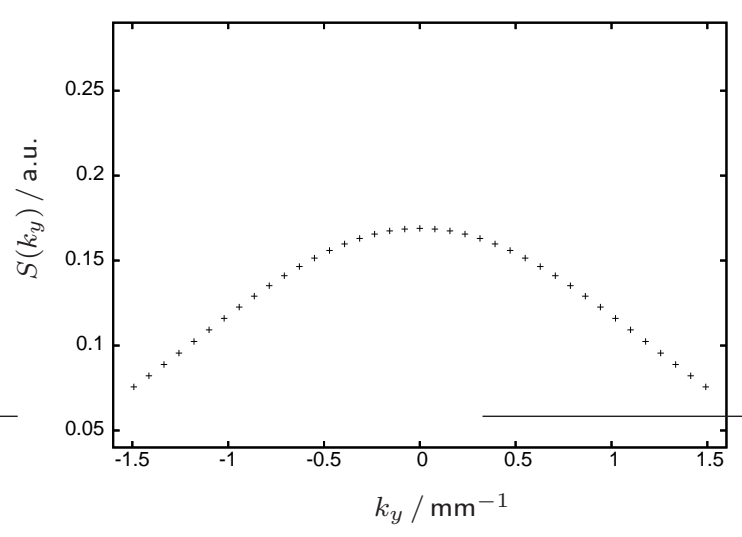

(e) FF, PPI: extern, PSF: Gauß, $\alpha(n)$ : variabel.

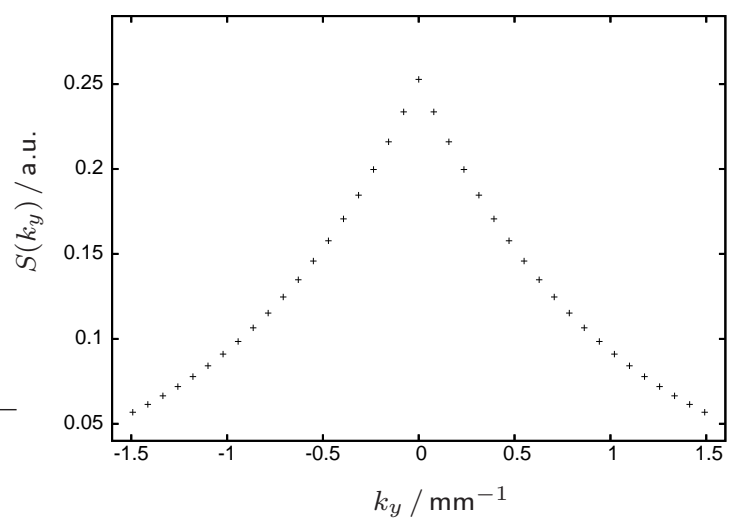

(b) FF, PPI: extern, PSF: Lorentz, $\alpha(n)$ : variabel.

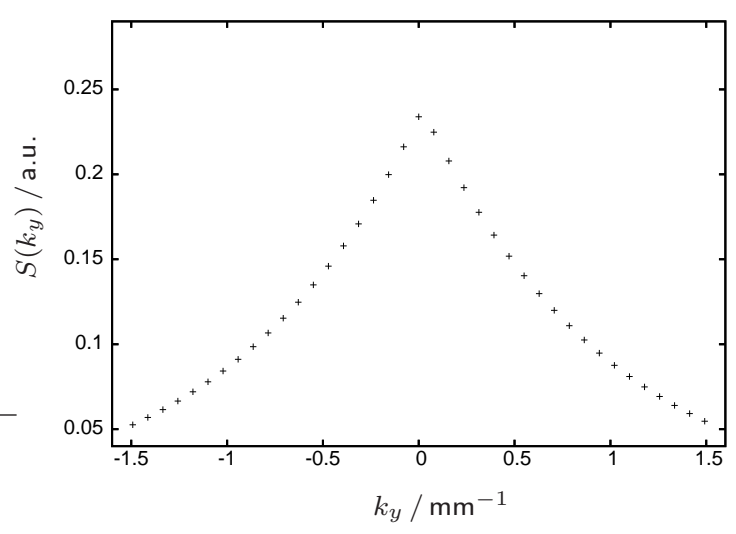

(d) FF, PPI: extern, PSF: Lorentz, $\alpha(n)$ : konstant.

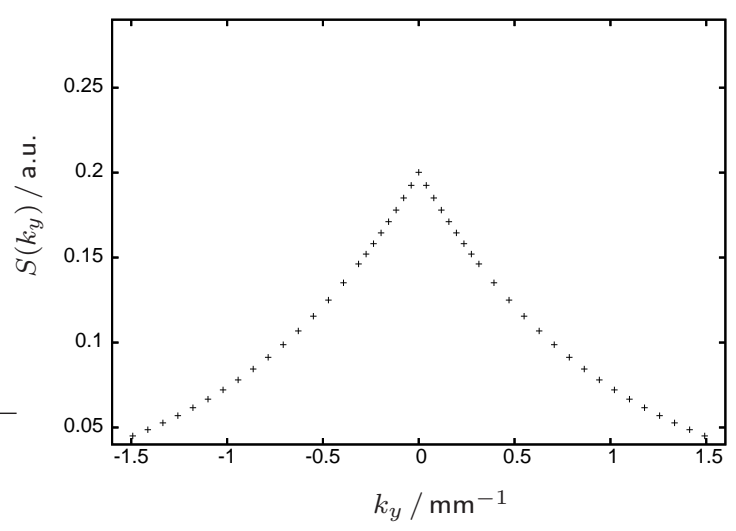

(f) FF, PPI: intern, PSF: Lorentz, $\alpha(n)$ : variabel.

Abbildung 4.6: Akquisitionsmethoden und Flipwinkelfunktionen. k-Raum-Abfall der verglichenen Kombinationen. Für Varianten mit PPI ist hier exemplarisch $R=2$. 
wobei ab $R=3$ eine Art Sättigungsverhalten einzutreten scheint.

Die Verwendung eines Gauß-Profils als PSF führt gegenüber der Lorentz-Variante mit variablen Flipwinkeln zu einem gravierenden SNR-Verlust. Diese verspricht ihrerseits im Vergleich mit der entsprechenden, nicht beschleunigten Full Fourier Methode zusätzlich zur Meßzeiteinsparung einen Qualitätsgewinn von ca. 16\% bei $R=2$ (FF+R2-e/Lorentz) bzw. $18 \%$ gegenüber der Referenzimplementation und maximal $26 \%$ bzw. $28 \%$ für $R=6$.

Bei der Kombination der PPI- mit der PF-Technik (externen Referenz) wurde ein PFFaktor von 6/8 gewählt, um gegenüber der reinen 5/8-Partial Fourier Messung eine ähnliche Anzahl von tatsächlich akquirierten Phasenkorrekturlinien zu erhalten. Der theoretische SNR-Verlauf weist diese Variante als der korrespondierenden, PPI-beschleunigten Full Fourier Methode unterlegen aus, was nicht unerwartet ist, denn die zusätzlich eingesparten Linien liegen im äußeren Bereich des k-Raums, für den sowieso vergleichsweise wenig Magnetisierung aufgewendet wird.

\subsubsection{Bandbreite}

Die durch parallele Bildgebung gewonnene Meßzeit kann in eine niedrigere Datenaufnahmebandbreite $(B W)$ reinvestiert werden, in der Hoffnung, damit gemäß Formel (3.12) das für die Diffusionssequenz so wichtige SNR eines Einzelbildes zu steigern und insbesondere den Rauschuntergrund zu reduzieren. Bei einer Verlängerung des Datenakquisitionsintervalls steht wegen der stärker ausgeprägten $T_{1}$-Relaxation eigentlich effektiv weniger Magnetisierung für die Bildgebung zur Verfügung und der maximal applizierte Flipwinkel ist reduziert. Weil gleichzeitig der Einfluß des Rauschens mit der Wurzel der Bandbreite verringert wird, sollte ein Maximum der SNR-Kurve zu erwarten sein. Ein niedrigerer $B W$-Wert geht aber bei der DW-SSSTEAM-Sequenz neben einem erhöhten $T R_{2}$ ebenso mit einer Ausdehnung des Zeitabschnitts vor dem zweiten 90'-Puls einher, kurz gesagt verlängert sich $T E_{\mathrm{STE}}$ (siehe Abbildung 3.5). Dadurch ist die Magnetisierung dem $T_{2}$-Zerfall vermehrt ausgesetzt, was als Korrekturfaktor in die Berechnung miteingehen muß. Die angeführten Abhängigkeiten lassen sich in der Beziehung

$$
\mathrm{SNR} \propto \sin \left(\alpha\left(n\left(k_{y}=0\right)\right)\right) e^{-T E_{\mathrm{STE}} / T_{2}} \sqrt{\frac{N_{\mathrm{ph}}^{\mathrm{acq}}}{B W}} .
$$

zusammenfassen. Die zulässigen Werte für $T E_{\mathrm{STE}}$ und damit auch die erlaubten Bandbreiten wurden mit der Siemens-Scannersoftware berechnet. Die hier durchgeführten SNR-Kalkulationen beruhen auf der Annahme eines $T_{2}$-Wertes von $65 \mathrm{~ms}$, der sich damit im unteren Bereich der Schätzungen für die Spin-Spin-Relaxationszeiten in der weißen Hirnsubstanz bei drei Tesla bewegt [85].

In [74] wurde für die DW-SSSTEAM-Sequenz bei einer Full Fourier Messung mit konstanten Flipwinkeln als optimale Einstellung $B W=180 \mathrm{~Hz} /$ Pixel angegeben und dieser Wert auf die Partial Fourier Akquisition übertragen. Diese Parameterwahl entspricht jedoch, wie man in Abbildung 4.8 sieht, für variable Flipwinkel nicht mehr dem Maximum der SNR-Funktion, das sich zu niedrigeren Bandbreiten verschiebt. Der optimale Wert ist insbesondere bei paralleler Bildgebung mit dem niedrigsten Reduktionsfaktor $R=2$ 
selbst bei $B W=100 \mathrm{~Hz} /$ Pixel scheinbar noch nicht erreicht, wo technische Probleme das weitere Verfolgen der funktionalen Abhängigkeiten verhinderten.

Hinsichtlich der Qualitätsverbesserung erlaubt die Methode der Bandbreitenverringerung im Gegensatz zur Mittelung von Magnitudenbildern in einem gewissen Rahmen eine nahezu stufenlose Kontrolle der Meßzeit. Außerdem wird nicht nur die Schätzung der Erwartungswerte der Pixelintensitäten verbessert, sondern auch der Rauschuntergrund unterdrückt und damit ein echter SNR-Gewinn erzielt (vgl. Abschnitt 3.3.2).

Die Bandbreitenerniedrigung unterliegt unter in-vivo Bedingungen aufgrund von Bewegungsartefakten gewissen Beschränkungen. So stellt zum Beispiel bei EKG-synchronisierten Messungen die inverse Herzfrequenz eine natürliche obere Schranke für die sinnvolle Meßzeit eines Einzelbildes dar. Bei einer Pulsrate von typischen 75 Schlägen pro Minute sind das $800 \mathrm{~ms}$, wovon bei Gehirnaufnahmen nach empirischen Untersuchungen ein etwa $200 \mathrm{~ms}$ langes systolisches Intervall erheblich von Pulsationsartefakten betroffen ist [86]. Es verbleiben also $600 \mathrm{~ms}$ zur Aufnahme. Eine weitere Einschränkung ist dadurch gegeben, daß CS-Artefakte (siehe Abschnitt 3.3.1) bei kleineren Bandbreiten stärker ausgeprägt sind.

\subsection{Implementierung und Einstellung der PPI-Parameter}

Die in Abschnitt 3.4.4 beschriebene Sequenz wurde um die neue Flipwinkelberechnung ergänzt und so modifiziert, daß die für die parallele Bildgebung benötigten k-RaumTrajektorien abgefahren werden können. Außerdem machen es die Änderungen an der Ansteuerungssoftware möglich, auch die Kombination von PPI (externe Referenzlinien) mit der Partial Fourier Technik zu realisieren. Allerdings ist der minimale PF-Faktor dabei aus technischen Gründen nach unten durch 6/8 beschränkt.

Für die Bildrekonstruktion stehen die von Siemens in Bibliotheksformat bereitgestellten Methoden GRAPPA und SENSE zur Verfügung, denen gegebenenfalls der POCSAlgorithmus nachgeschaltet wird, dessen Umsetzung auf der verwendeten Plattform Bestandteil einer vorangegangenen Promotionsarbeit [74] war.

Es sollte darauf hingewiesen werden, daß bereits im Rahmen zweier Konferenzen Veröffentlichungen zur Kombination von paralleler Bildgebung mit Single-Shot STEAM Sequenzen existieren [87, 88]. Allerdings benutzen die Autoren anscheinend konstante Flipwinkel und sie wenden die Technik auch nicht auf die Diffusionstensorbildgebung an.

Für die PPI-Rekonstruktion erwies sich GRAPPA gegenüber SENSE (zumindest in der vorliegenden Realisierung) bei der Bildqualität, speziell im Hinblick auf Geisterartefakte, als überlegen. Sämtliche experimentellen Resultate der vorliegenden Arbeit beruhen deshalb auf diesem Algorithmus. Die zur Messung eingesetzte Phased Array Spule mit acht Empfangskanälen erlaubt zwar theoretisch einen maximalen Reduktionsfaktor von $R=8$, jedoch wird eine Aufnahmeebene durch die zylindrische Anordnung der Spulenelemente typischerweise nur einen Überlapp mit vier unabhängigen Sensitivitätsprofilen haben. Der verwendbare Wertebereich für $R$, sowie die minimal benötigte Anzahl der Referenzlinien wurden visuell anhand der auftretenden Rekonstruktionsartefakte bestimmt. Abbildung 4.9 zeigt exemplarisch die Einstellung des Parameters $N_{\text {ref }}$. 


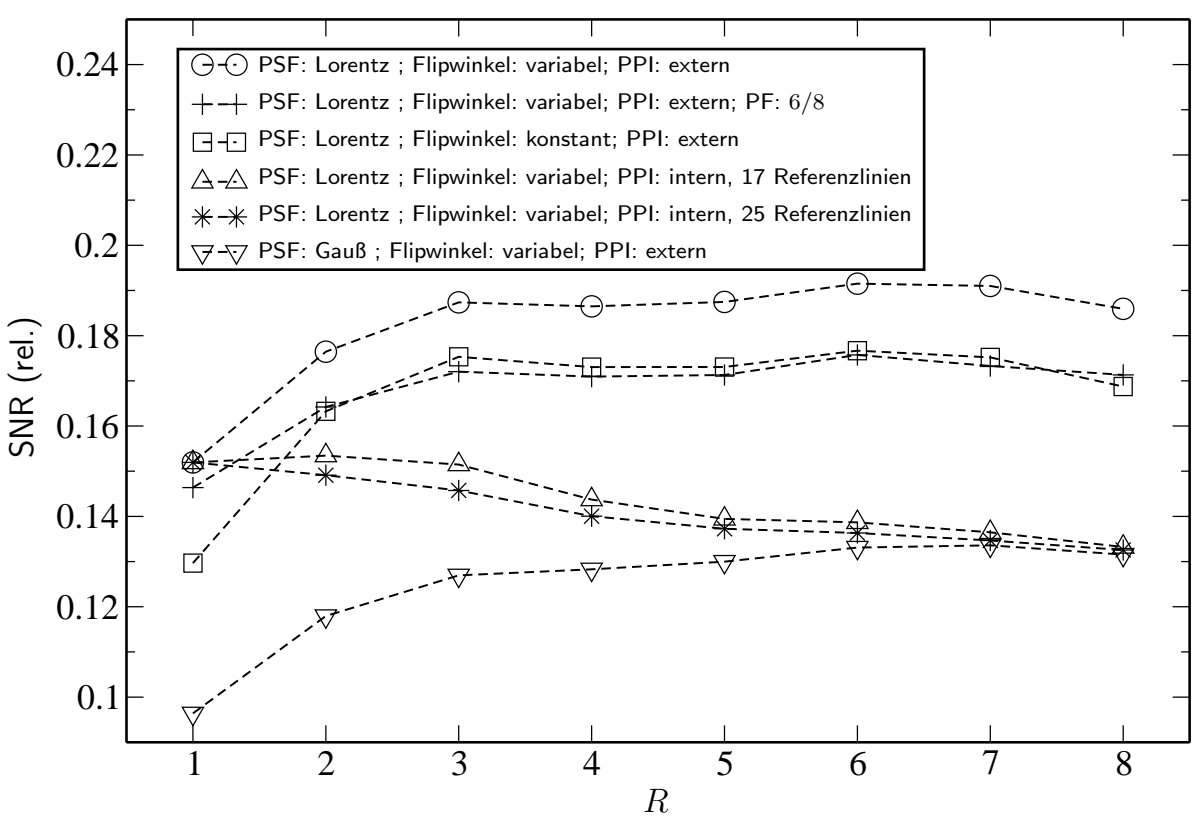

Abbildung 4.7: SNR-Verhalten bei PPI-Beschleunigung. Vergleich des theoretisch vorhergesagten relativen SNR (Gleichung (4.11)) bei verschiedenen Meßmethoden und PPIParametern. Soweit nicht anders angegeben, entsprechen die Kurven Full Fourier Akquisitionen.

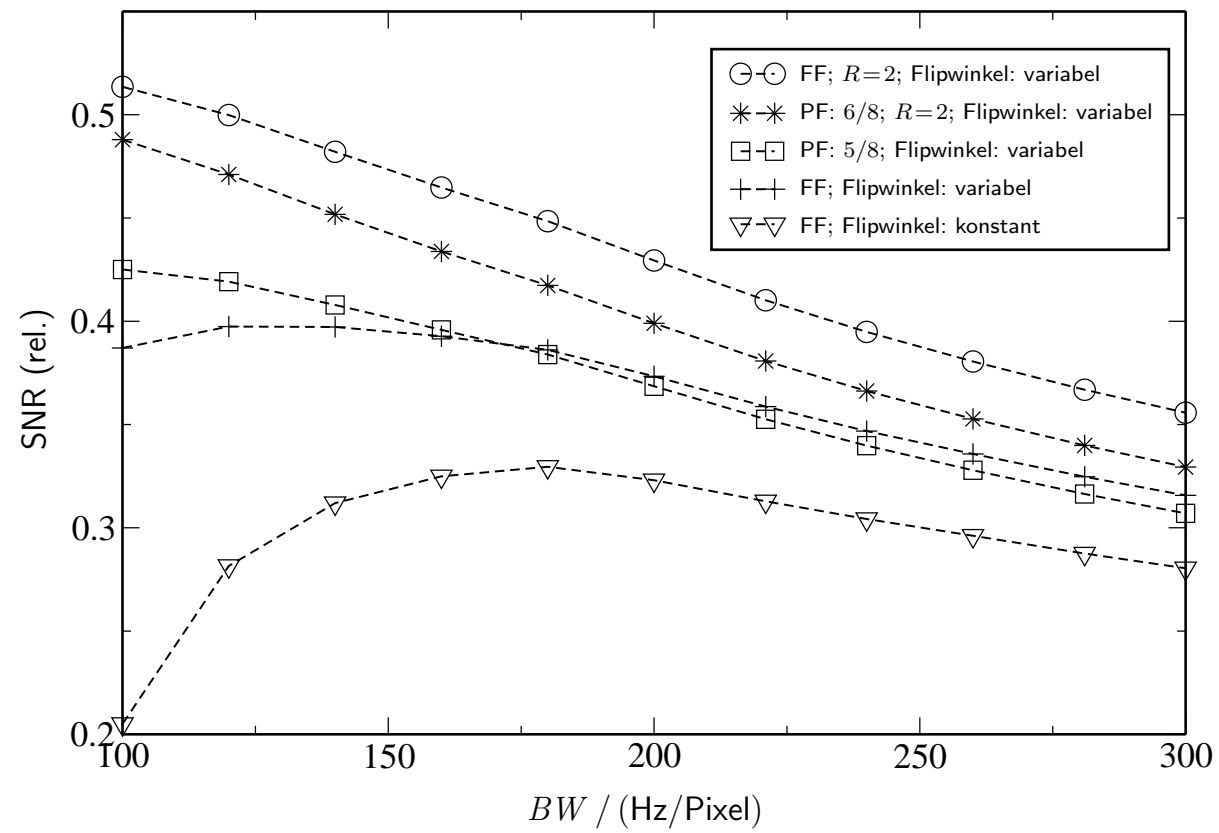

Abbildung 4.8: Optimierung der Bandbreite. Theoretische funktionale Abhängigkeit des relativen SNR von der Bandbreite (Gleichung (4.12)) für eine lorentzförmige PSF bei unterschiedlichen Aufnahmetechniken. Ist $R>1$, handelt es sich um PPI mit externen Referenzlinien. 


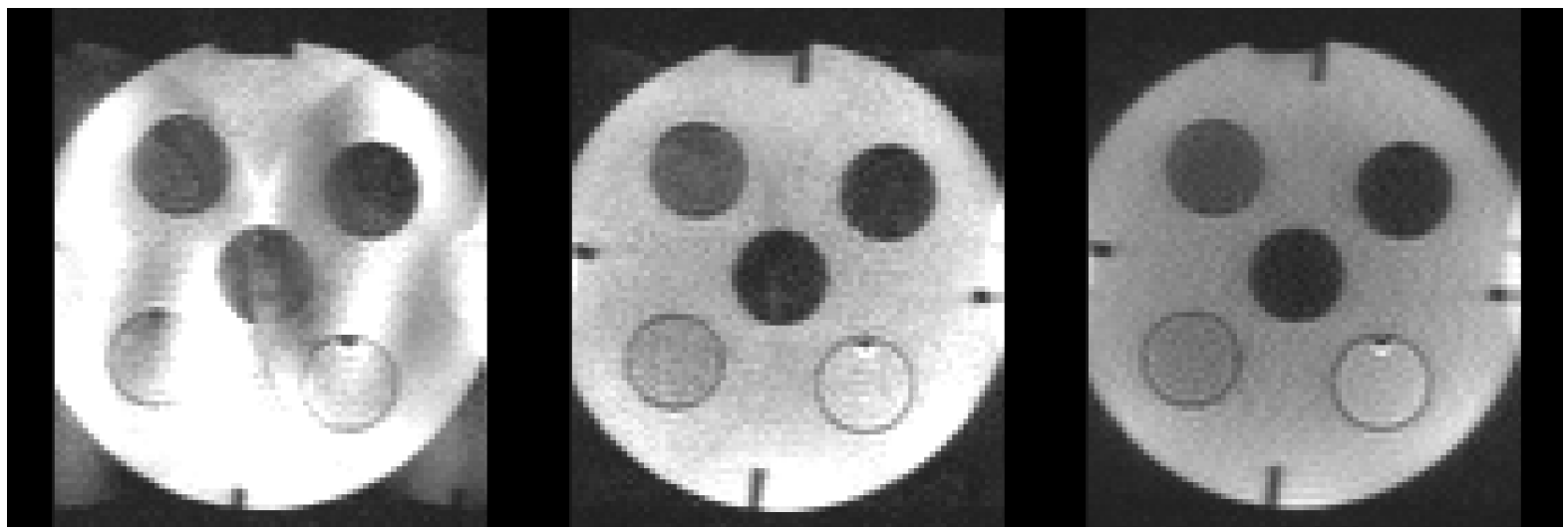

(a) $N_{\text {ref }}=9$ Autokalibrierungslinien (b) $N_{\text {ref }}=17$ Autokalibrierungslinien (c) $N_{\text {ref }}=25$ Autokalibrierungslinien

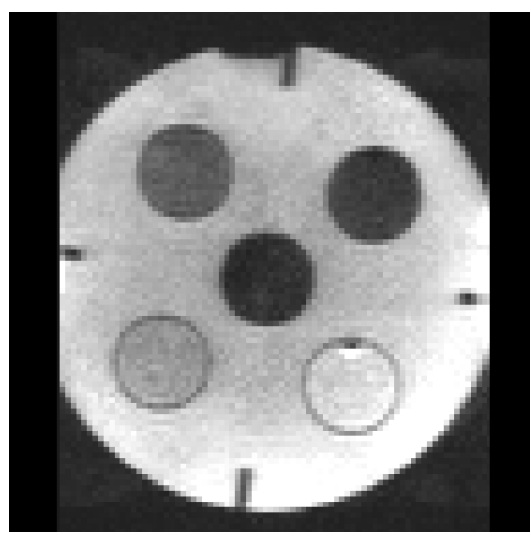

(d) $N_{\text {ref }}=25$ externe Referenzlinien

Abbildung 4.9: PPI-Parameter. (oben) Wahl der Spulenkalibrierungslinien $N_{\text {ref }}$ bei PPI mit Autokalibrierung anhand einer Phantommessung. Erwartungsgemäß haben Bilder mit weniger Referenzlinien höhere Bildintensitäten, sie leiden aber unter rekonstruktionsbedingten Geisterbildern entlang der reduziert akquirierten Koordinate. (unten) Zum Vergleich ist in Abbildung 4.9(d) ein Aufnahme mit 25 externen Referenzlinien abgebildet.

Es stellt sich heraus, daß unter den gegebenen Voraussetzungen nur der minimale Reduktionsfaktor $R=2$ sinnvoll verwendet werden kann, da ansonsten periodische PPIArtefakte und übermäßige Rauschverstärkung die Bildqualität zu stark beeinträchtigen. In diesem Fall sind 25 Referenzlinien nötig um bei autokalibrierten Messungen sichtbare Rekonstruktionsfehler nahezu auszuschließen; bei separater Spulenprofilbestimmung verringert sich die Stärke der immer noch (wenn auch schwach) vorhandenen Bildfehler ab diesem Wert nicht mehr. Ihre Ausprägung ist vergleichbar zu einer Aufnahme mit siebzehn internen Autokalibrierungslinien. Um eine SNR-Gewinn gegenüber der Referenzimplementation aus Abschnitt 3.4.4 zu erzielen, wird deshalb im folgenden trotz 
leichter remanenter Geister-Artefakte für die PPI-i-Meßvariante die Einstellung $N_{\text {ref }}=17$ verwendet.

\subsection{Qualitativer Methodenvergleich}

Für eine erste qualitative Einschätzung der Ergebnisse genügt erfahrungsgemäß bei der Diffusionstensorbildgebung nicht allein die Betrachtung der Rohdaten. Es ist darüberhinaus notwendig, zusätzlich auch die daraus gewonnenen skalaren Karten, z. B. der fraktionellen Anisotropie, zu berücksichtigen.

Wegen des erheblichen Zeitaufwands einer Diffusionsmessung war es für den Methodenvergleich nicht möglich alle Meßvarianten am selben Probanden anzuwenden. Obwohl versucht wurde, durch eine ähnliche Schichtorientierung und -position Vergleichbarkeit herzustellen, muß klar sein, daß wegen der interindividuellen anatomischen Unterschiede, durch Bewegungen eines Probanden zwischen zwei Akquisitionen und aufgrund von Hirnpulsationen die Darstellungen einzelner Hirnwindungen nicht sinnvoll detailliert miteinander verglichen werden können. Aussagekräftiger sind das Ausmaß des Rauschens in FA-Karten und die Häufigkeit von negativ definiten Diffusionstensoren (siehe Abschnitt 3.3.2), die bei der rauschbehafteten Bestimmung kleiner Diffusionskonstanten in Erscheinung treten können, also insbesondere auch in hochstrukturierter weißer Hirnsubstanz orthogonal zur Faserrichtung (vgl. [89]).

Für einen visuellen Vergleich (Abbildung 4.10) werden an dieser Stelle

1. die unbehandelten Rohdaten, zur Abklärung von grundlegenden Bildartefakten,

2. die direkt aus den Rohdaten berechneten Anisotropiewerte und

3. Anisotropiekarten auf der Basis von geglätteten Rohdaten (lineare Interpolation auf $1 \times 1 \times 1 \mathrm{~mm}^{3}$, 3D-Gauß-Filter mit $\sigma=1 \mathrm{~mm}$ )

herangezogen.

Als Ergebnis kann unter anderem festgehalten werden, daß die Verwendung interner Referenzlinien (FF+R2-i) gegenüber der in Meßzeit und Signalstärke vergleichbaren Partial Fourier-Methode (PF 5/8) keine nennenswerten Vorteile, aber Einbußen in der Bildqualität mit sich bringt. Das zeigt sich besonders in der stark erhöhten Anzahl negativ definiter Diffusionstensoren auf den ungeglätteten Daten. Aufnahmemethoden mit externen Referenzlinien erscheinen dagegen aussichtsreicher, weil sie, mit Ausnahme der Meßvariante mit Gaußscher PSF (FF-R2-e/Gauß, aus diesem Grund ebenfalls ein Ausschlußkandidat), theoretisch einen beträchtlichen Signalanstieg gegenüber der Referenzsequenz zu verbuchen haben.

Die Kombination der Partial Fourier Technik mit PPI-e (PF 6/8+R2-e) ist theoretisch bei gleicher Bandbreite, vom SNR pro Bild betrachtet, der alleinigen Beschleunigung durch parallele Bildgebung unterlegen (siehe Abbildung 4.7), was sich auch qualitativ in den Bildern niederschlägt. Allerdings reduziert sich die (totale) Aufnahmezeit gegenüber der Referenzsequenz um ca. 34\%. Neben dem prinzipiellen Problem, daß man bei dieser Variante einige für die PF-Rekonstruktion benötigte zentrale Phasenkorrekturlinien 
nur schätzt und nicht misst, hat der verwendete POCS Algorithmus anscheinend unvorteilhafte Auswirkungen auf den Rauschuntergrund, ein Thema des nächsten Abschnitts. Deswegen wurde auf eine weitere Untersuchung von PF 6/8+R2-e, also auch auf eine mögliche Anpassung der Bandbreite, verzichtet.

Einen Zeitgewinn von $17 \%$ bei augenscheinlich mit der Ausgangssequenz vergleichbarer Güte bietet dagegen die beschleunigte Full Fourier Meßvariante mit externen Referenzlinien und lorentzförmiger PSF (FF+R2-e/Lorentz). Die Zahl der auftretenden negativ definiten Diffusionstensoren ist dabei um ca. 9\%, bei einer Bandbreite von $130 \mathrm{~Hz} /$ Pixel und gleicher Aufnahmedauer wie bei der Referenzimplementation sogar um ca. $21 \%$ reduziert.

\subsection{Quantifizierung der Datenqualität}

Das am häufigsten zur quantitativen Bewertung herangezogene Qualitätsmerkmal eines NMR-Bildes ist sein Signal-zu-Rausch-Verhältnis. Bei der experimentellen Überprüfung der theoretischen Vorhersagen aus Abschnitt 4.3 stellt sich das Problem, daß das SNR in Aufnahmen mit Phased Array Spulen a priori keine absolute Größe darstellt, denn sowohl Intensität als auch Rauschcharakteristik sind räumlichen Schwankungen unterworfen. Der variable Rauschuntergrund ist inbesondere auch charakteristisch für mit PPI-Algorithmen rekonstruierte Bilder (siehe Gleichung (4.4)). Deshalb können die in Abschnitt 3.3.2 angeführten Meßverfahren nicht naiv übertragen werden. Desweiteren ist es bei in-vivo Aufnahmen schwierig, Bereiche homogenen Gewebes zu definieren. Aus den angeführten Gründen erscheint es sinnvoll, eine voxelbasierte Berechnungsstrategie $\mathrm{zu}$ verfolgen.

Zuerst sollen die numerisch über $\sin \left(\alpha\left(n\left(k_{y}=0\right)\right)\right)$ geschätzten Bildintensitäten mit den in-vivo erzielten Daten verglichen werden. Dabei findet das folgende Verfahren Anwendung: während der Meß-Sitzung wird zunächst die diffusionsgewichtete Referenzsequenz eingesetzt. Außerdem umfaßt das Protokoll im Anschluß die Aufnahme ungewichteter Datensätze mit den zu vergleichenden Akquisitionsmethoden. In einem Nachbearbeitungsschritt erfolgt anhand der auf dem Diffusionsdatensatz berechneten FA-Werte die Segmentierung der weißen Hirnsubstanz (FA $\geq 0,2)$. Durch Übertragung dieser Maske auf die ungewichteten Aufnahmen ist ein voxelweiser Vergleich der Bildwerte der einzelnen Methoden in der Zielregion in Bezug auf die Referenzsequenz und eine Gegenüberstellung der Ergebnisse mit den theoretischen Vorhersagen möglich.

An die Histogramme der Intensitätsdifferenzen und der Bildwerte in der weißen Hirnsubstanz lassen sich im Bereich des Maximums Gauß-Verteilungen anfitten. Mit den Abszissenwerten der Extrema kann man dann eine relative Quantifizierung durchführen. Teilweise auftretende Symmetrieabweichungen sind auf den Rauschuntergrund, Artefakte durch Hirnpulsationen und Inhomogenitäten in der weißen Substanz (biologisch immanent oder wegen Spulenprofilmodulationen) zurückzuführen.

Die Aufstellung in Tabelle 4.1 belegt die qualitative Übereinstimmung der experimentellen Daten mit den theoretischen Vorhersagen, allerdings wird der Absolutbetrag der Signaländerung in einigen Fällen um 4-8\% überschätzt. Eine mögliche Ursache dafür wäre 


\begin{tabular}{l|ll|ll}
\hline & \#Linien & $\begin{array}{c}\text { Aufnahmezeit } \\
\text { pro Bild (ms) }\end{array}$ & \multicolumn{2}{|c}{ Relative Signaländerung } \\
experimentell & theoretisch \\
\hline PF 5/8 & 50 & 466 & Referenz & \\
FF (konst. Flipwinkel) & 80 & 700 & $-0,240 \pm 0,001$ & $-0,321$ \\
FF (var. Flipwinkel) & 80 & 700 & $-0,162 \pm 0,001$ & $-0,205$ \\
FF+R2-e/Lorentz & 39 & 380 & $+0,290 \pm 0,001$ & $+0,323$ \\
FF+R2-e/Gauß & 39 & 380 & $-0,133 \pm 0,001$ & $-0,115$ \\
PF 6/8+R2-e & 29 & 302 & $+0,382 \pm 0,001$ & $+0,428$ \\
FF+R2-i (17 Ref.Linien) & 47 & 442 & $+0,038 \pm 0,001$ & $+0,048$ \\
FF+R2-i (25 Ref.Linien) & 51 & 474 & $-0,028 \pm 0,001$ & $-0,022$ \\
\hline
\end{tabular}

Tabelle 4.1: Signalstärke. Vergleich der experimentellen relativen Bildintensitätsveränderungen der eingesetzten Meßmethoden gegenüber der Referenzsequenz mit den theoretische Vorhersagen. Man beachte, daß es sich um eine Gegenüberstellung der Signalstärken, nicht aber der SNR-Werte handelt, die nach Formel (4.11) zusätzlich mit der Anzahl der aufgenommenen Linien skalieren. Die Auflistung sagt also noch nichts über die Güte der entsprechenden Bilder aus.

eine nicht konstante Flipwinkelverteilung über dem Zielvolumen. Der Vergleich der Messung mit Gaußscher Punkt-Transfer Funktion anhand des Signals in der k-Raum-Mitte ist eigentlich zur Abschätzung der relativen Intensitätsveränderung nicht zulässig, weil die Energie der entsprechenden Bilder anders verteilt ist als bei der Referenzaufnahme.

Nach den bisher gewonnenen Erkenntnissen hat sich die Meßvariante FF+R2-e/Lorentz als sinnvolle Grundlage weiterer Untersuchungen erwiesen. Um das SNR dieser Sequenz mit der Referenzimplementation vergleichen zu können, wurden zehn zusätzliche ungewichtete Aufnahmen mit beiden Methoden gemacht. Die Daten erlauben es, in den durch die FA-Maske selektierten Voxeln jeweils eine Standardabweichung zu berechnen. Das ist insofern sinnvoll, als daß nach Abschnitt 3.3.2 eine Gauß-Kurve das statistische Verhalten der gemessenen Intensitäten in Magnitudenbildern für hinreichend große Werte gut approximiert, und die Varianz $\sigma^{2}$ der des Meßrauschens entspricht. Dabei wird hier vorausgesetzt, daß diese Eigenschaften auch in PPI-rekonstruierten Bildern erfüllt sind.

Der Vergleich der Histogramme der SNR-Verteilungen von PPI- und Referenzsequenz in Abbildung 4.11 macht deutlich, daß die Rauschverstärkung infolge der Rekonstruktion den theoretischen Vorteil der durch parallele Bildgebung beschleunigten Meßvariante wieder zunichte macht, allerdings auch keine Qualitätseinbußen trotz der verkürzten Meßzeit festzustellen sind. Nimmt man das Maximum der Kurven als Bezugspunkt, so liegt das SNR der beiden Aufnahmemethoden ungefähr bei 7 .

Im Gegensatz zu der hier propagierten Vorgehensweise ergibt die Anwendung der Technik aus Abschnitt 3.3.2 zur Bestimmung des Signal-zu-Rausch-Verhältnisses über ROIMittelwerte und die Eigenschaften des Rauschuntergrundes (siehe Gleichung (3.13b)) für PF 5/8 ein SNR von 5,1 und für FF+R2-e einen Wert von ca 8. Dieses Resultat würde auf einen massiven Güte-Unterschied der beiden Sequenzen hindeuten, der jedoch weder mit dem voxelbasierten Verfahren noch anhand des visuellen Eindrucks bestätigt werden 
kann, abgesehen von der Differenz in der Anzahl negativ definiter Diffusionstensoren. Eine genauere Untersuchung zur Auflösung des Widerspruchs zeigt, daß die auf die konventionelle Weise bestimmten Werte für die mittlere Intensität bei PF 5/8 und FF+R2-e, sowie von $\sigma$ bei $\mathrm{FF}+\mathrm{R} 2$-e mit den Maxima der entsprechenden Histogramme gut übereinstimmen. Dagegen wird die Standardabweichung bei PF 5/8 durch die Messung der mittleren Bildintensität in signalfreien Regionen irrigerweise um 58\% gegenüber dem durch die Ensemble-Betrachtung (Abbildung 4.12) ermittelten Wert überschätzt. Die genaue Ursache für diese anomale Erhöhung des Rauschuntergrundes ist unklar, könnte aber mit der POCS-Rekonstruktion zusammenhängen.

\subsection{Diskussion}

In diesem Kapitel konnte gezeigt werden, daß durch parallele Bildgebung eine verlustfreie Beschleunigung von DW-SSSTEAM gegenüber der Referenzsequenz möglich ist. Das Einsparen von Meßzeit durch das Überspringen von k-Raum-Linien resultiert zwar nach Formel (4.11) in einer Vergrößerung des Bildrauschens, was sich auch anhand von Abbildung 4.12 nachvollziehen läßt. Trotzdem führt diese Tatsache hier nicht zu einer Verschlechterung des SNR (siehe Abbildung 4.11), weil wegen der ausgelassenen Auslesepulse die ihnen in einer unbeschleunigten Aufnahme ursprünglich zugedachte longitudinale Magnetisierung über eine auflösungswahrende, verallgemeinerte Flipwinkelfunktion umgewidmet, und zur Verbesserung des Signals eingesetzt werden kann. Dasselbe Argument trifft natürlich auch auf die Partial Fourier Technik zu. Allerdings hängt der kompensierende Effekt bei der gewählten lorentzförmigen Punkt-Transfer Funktion stark von der Lage der eingesparten Linien ab. Er ist umso größer, je zentraler die entsprechenden k-Raum-Positionen sind, denn auf diese Stellen entfällt normalerweise der Hauptteil der verfügbaren Magnetisierung. Die Art des Datenreduktionsschemas (vergleiche Abbildungen 3.9 und 4.1(a)) begünstigt deshalb PPI-e im Vergleich zu Partial Fourier, jedenfalls bei den gewählten Parametern. Obwohl die neue Meßvariante FF+R2-e also nach der numerischen Berechnung trotz der Aufnahme von nur 39 Linien der PF 5/8-Sequenz mit ihren 50 Linien sogar überlegen ist (siehe Abbildung 4.8), halten sich unter den gegebenen experimentellen Voraussetzungen, d. h. Spulengeometrie und Rekonstruktionsalgorithmus, die Rauschverstärkung und der Signalgewinn die Waage, so daß im Endeffekt eine Meßzeitverkürzung von $17 \%$ bei gleichbleibender Qualität zu verzeichnen ist. Ein weiteres Resultat dieses Kapitels ist die Erkenntnis, daß das Optimum der Aufnahmebandbreite zumindest im Hinblick auf das SNR pro Bild gerade auch bei k-Raum-reduzierten Aufnahmen weit unter dem in der Referenzimplementation voreingestellten Wert von $180 \mathrm{~Hz} /$ Pixel liegt.

Zur Bestimmung des Signal-zu-Rausch Verhältnisses kam eine auf Ensemble-Messungen beruhende voxelbasierte Methode zum Einsatz. Mit einiger Berechtigung kann man behaupten, daß dieser Zugang den traditionell benutzten Ansätzen überlegen ist. Allerdings spielt dabei die räumliche Inhomogenität der zu bestimmenden Größen $I$ und $\sigma$, derentwegen das Verfahren überhaupt erst ausgearbeitet wurde, hier nicht die entscheidende Rolle. Vielmehr ließ sich mit der Methode unbeabsichtigt ein Rekonstruktionsar- 
tefakt in den mit POCS berechneten Aufnahmen aufdecken. Die in den PF 5/8-Bildern festgestellte Anomalie äußert sich in einem gegenüber dem theoretischen Niveau (3.13b) inkrementierten Rauschuntergrund, der anscheinend nicht immer den Gesetzmäßigkeiten der Rayleigh-Verteilung folgt. Diese Beeinträchtigung der Aufnahmen der Partial Fourier Variante kann sehr wahrscheinlich auch für die empirisch beobachtete Differenz in der Anzahl der negativ definiten Diffusionstensoren im Vergleich mit der FF+R2e-Akquisition verantwortlich gemacht werden, die auftritt, obwohl beide Sequenzen eigentlich das gleiche SNR haben. Das Artefakt verhindert die korrekte Bestimmung der Standardabweichung mit der für Magnitudenbilder üblichen Prozedur. 

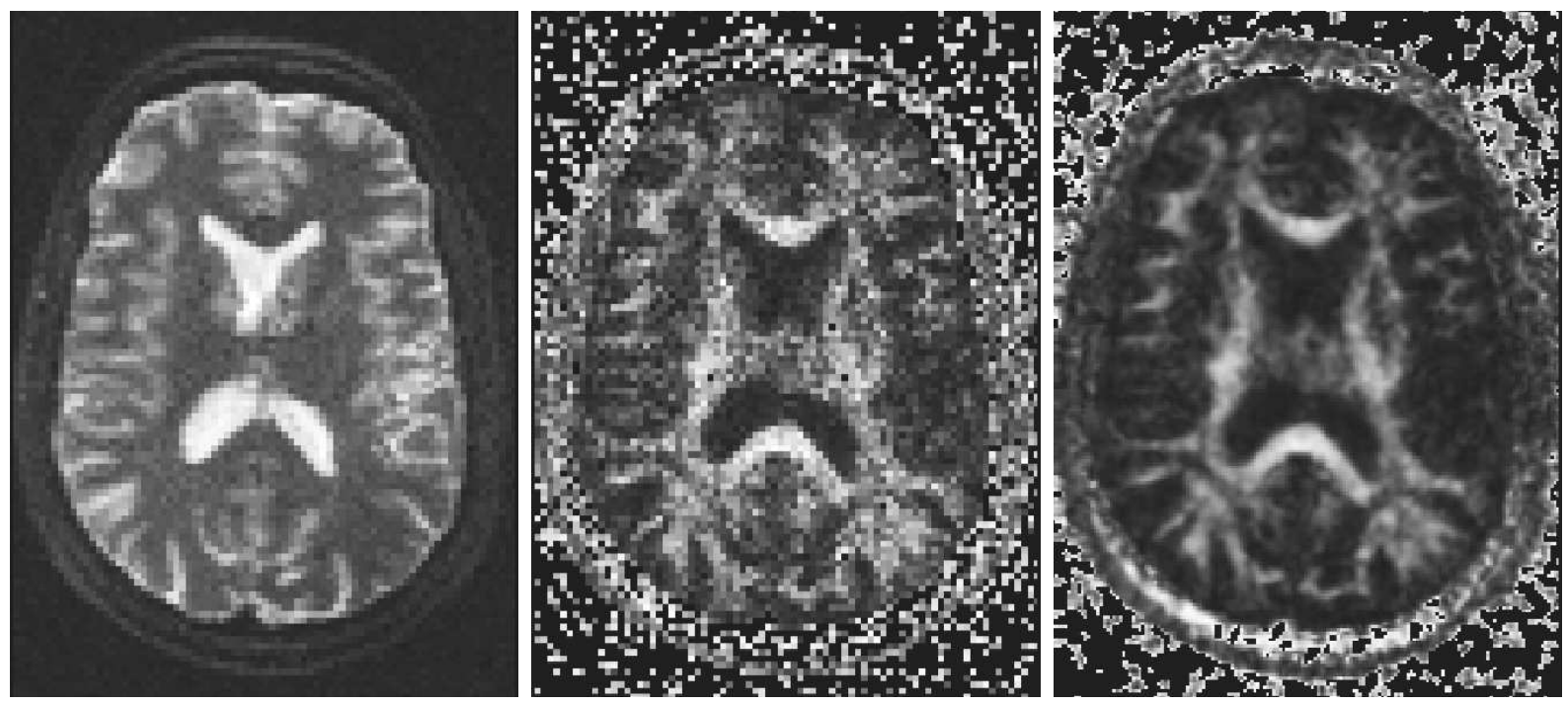

(a) PF $5 / 8$
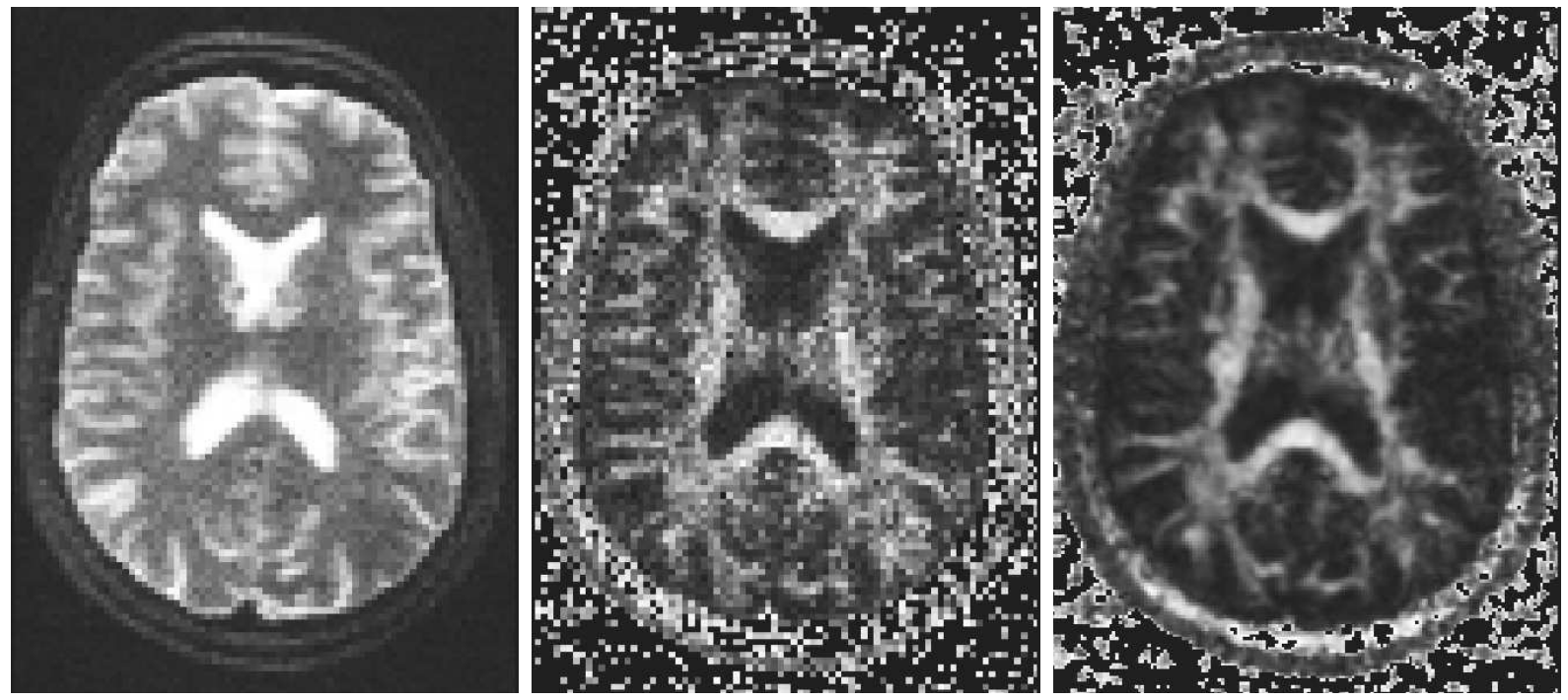

(b) $\mathrm{FF}+\mathrm{R} 2-\mathrm{e} /$ Lorentz

Abbildung 4.10: Visuelle Bewertung der Bildqualität unterschiedlicher Akquisitionsmethoden. Wenn nicht explizit notiert, lag die Aufnahmebandbreite bei $B W=$ $180 \mathrm{~Hz} /$ Pixel. (links) Nicht diffusionsgewichtete Aufnahme, (Mitte) FA-Karte, (rechts) FAKarte mit geglätteten Rohdaten 

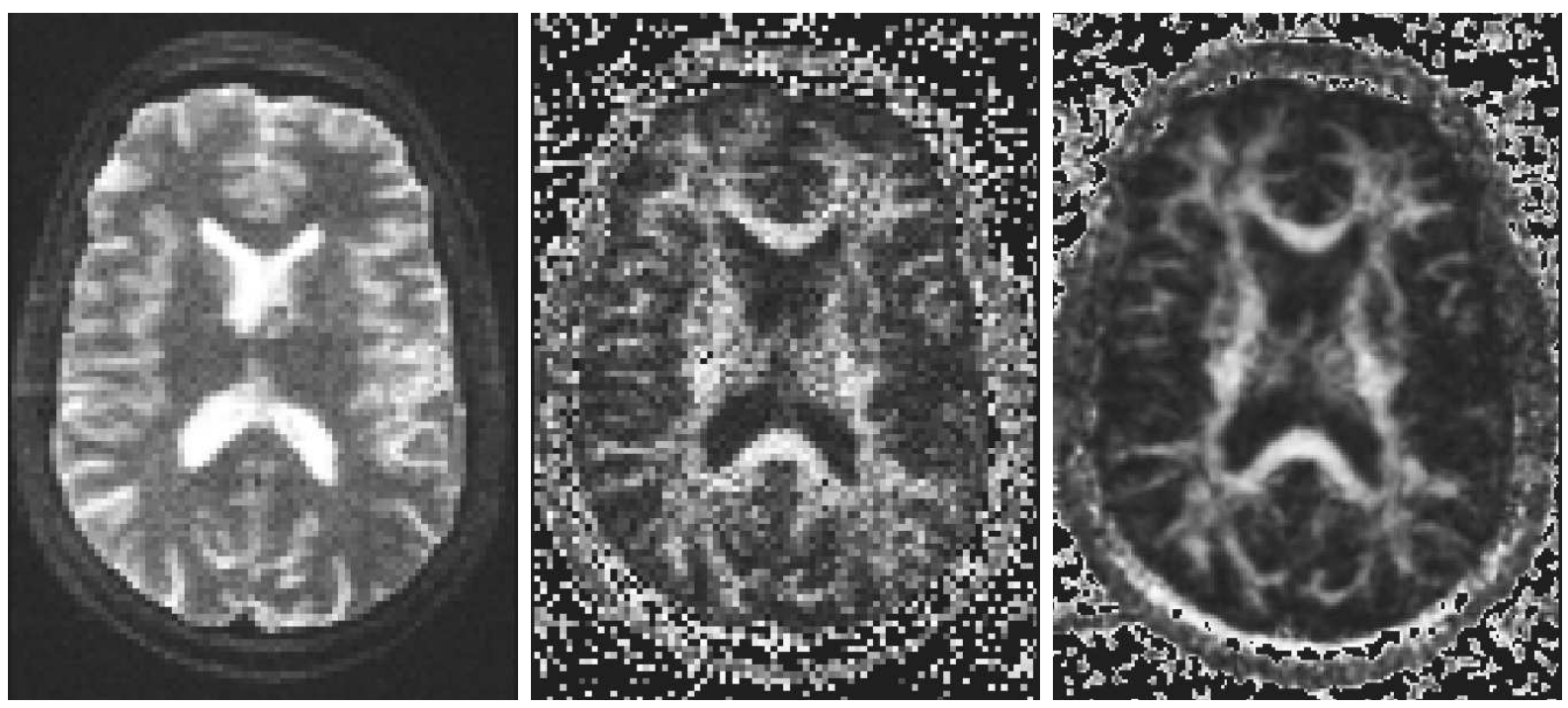

(c) $\mathrm{FF}+\mathrm{R} 2-\mathrm{e} /$ lorentz, $B W=130 \mathrm{~Hz} /$ Pixel
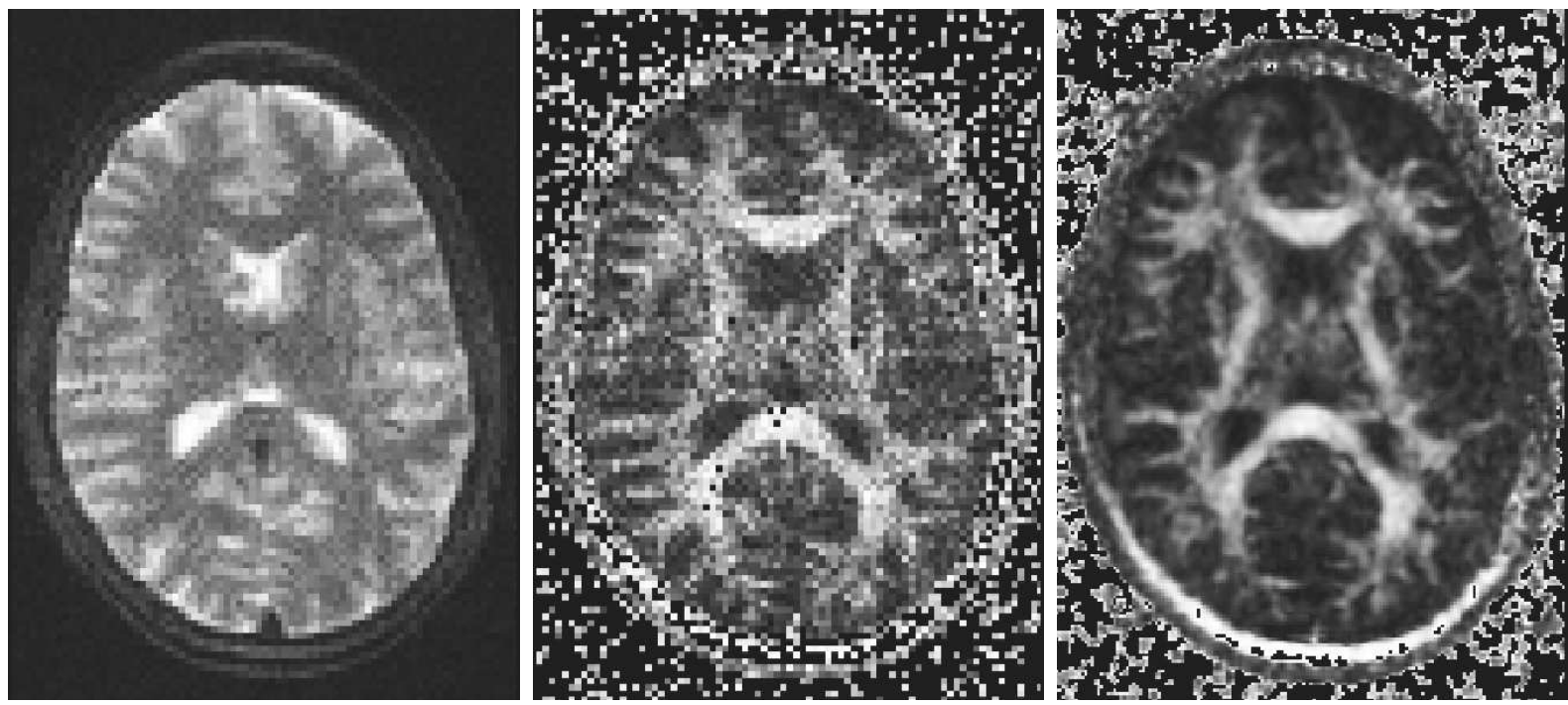

(d) $\mathrm{PF} 6 / 8+\mathrm{R} 2-\mathrm{e}$

Abbildung 4.10: (fortgesetzt) 

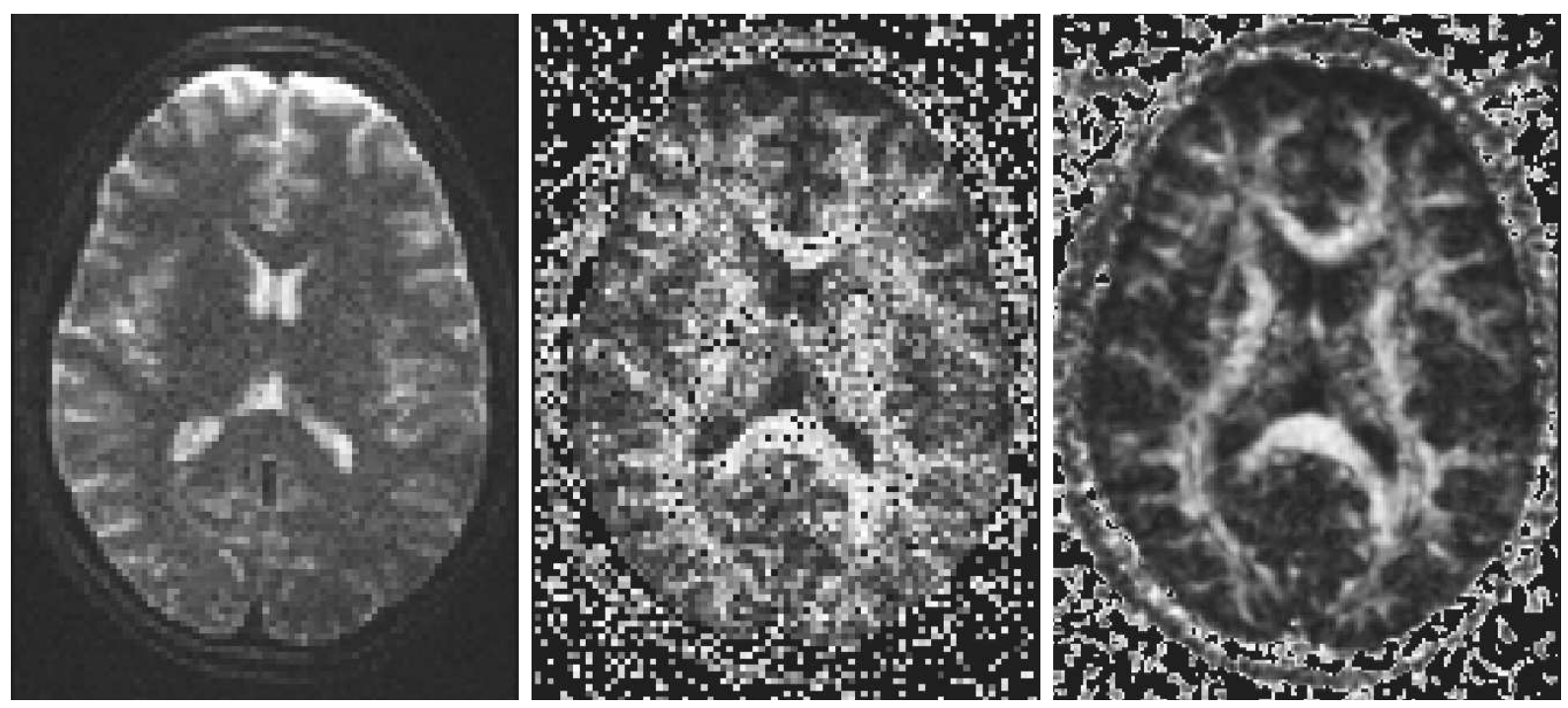

(e) $\mathrm{FF}+\mathrm{R} 2-\mathrm{e} / \mathrm{Gauß}$
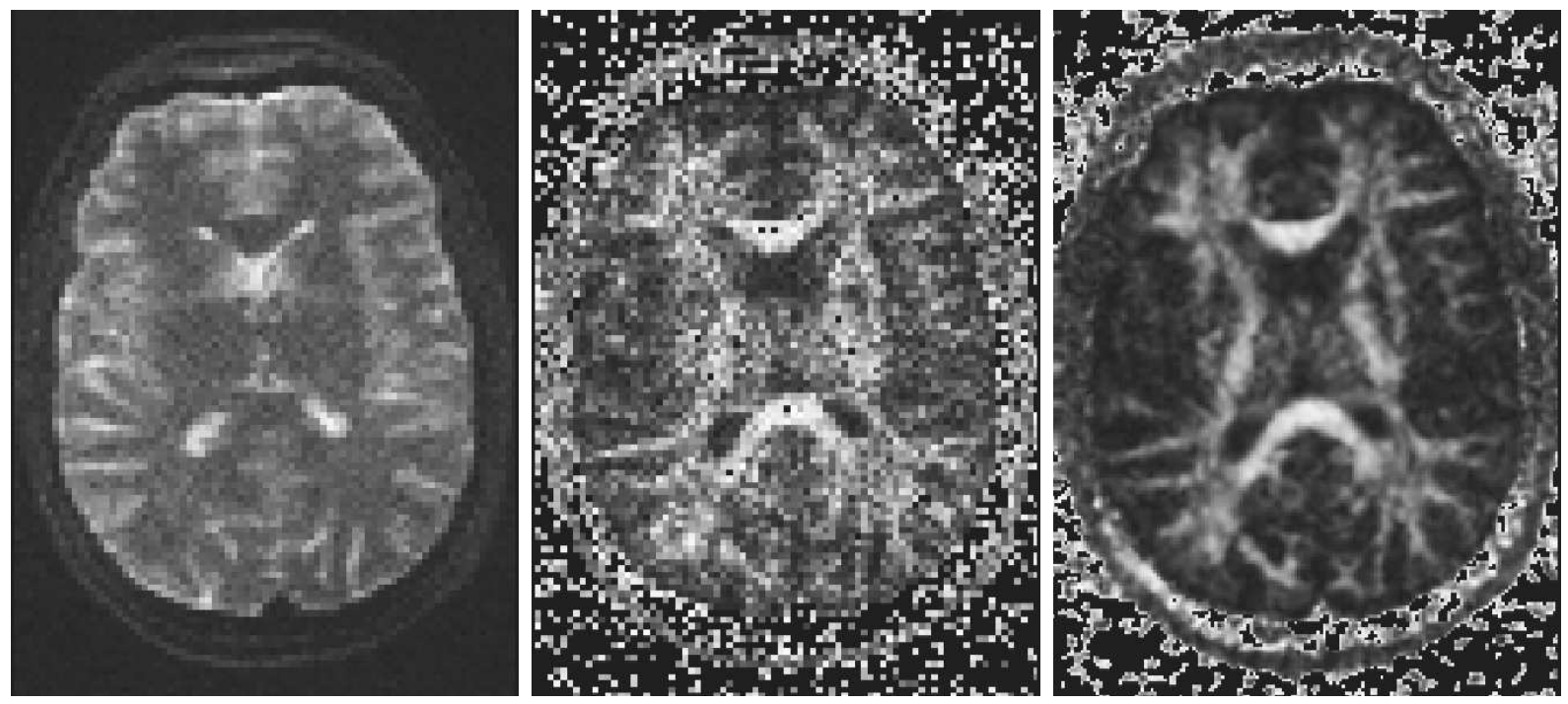

(f) $\mathrm{FF}+\mathrm{R} 2-\mathrm{i}, N_{\text {ref }}=17$

Abbildung 4.10: *

(fortgesetzt) 


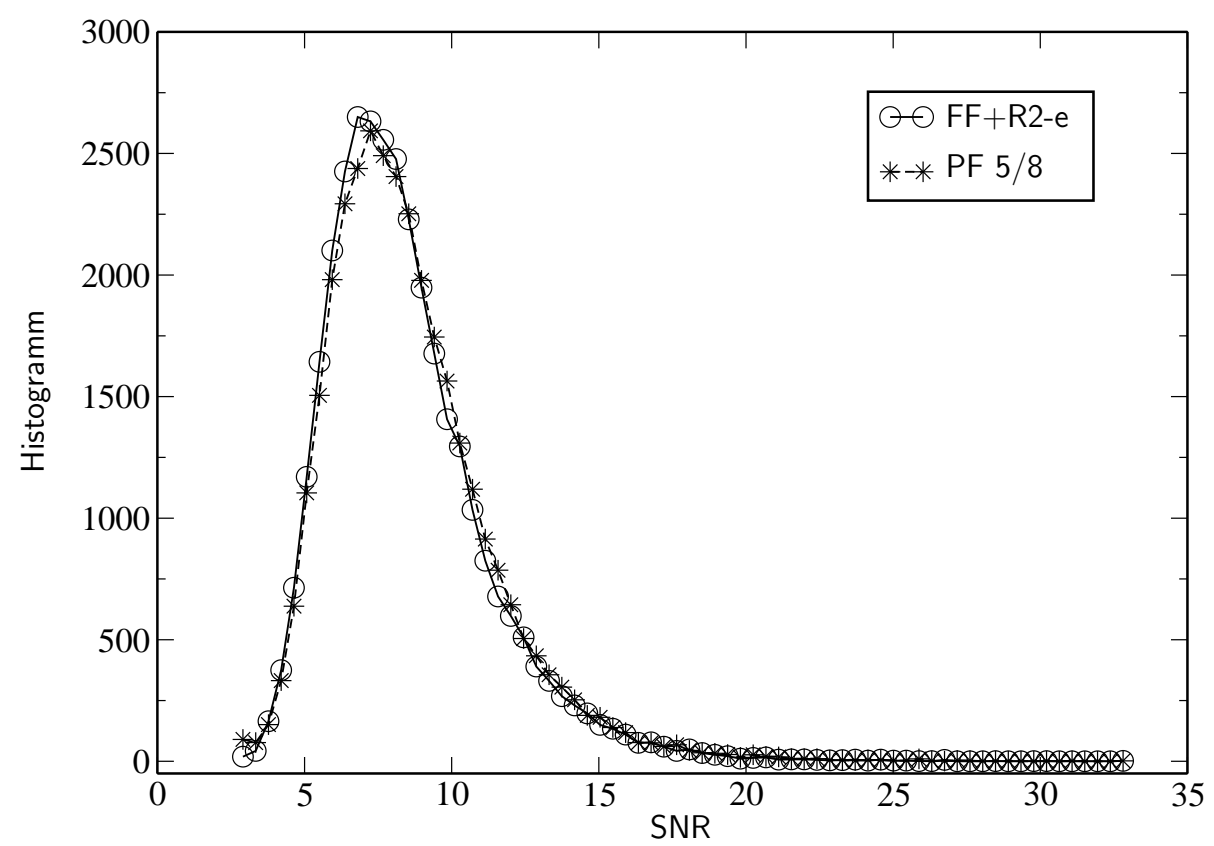

Abbildung 4.11: SNR-Histogramm. Quantitativer Vergleich der Datenqualität von FF+R2-e mit der Referenzimplementation über eine voxelbasierte Ensemble-Messung.

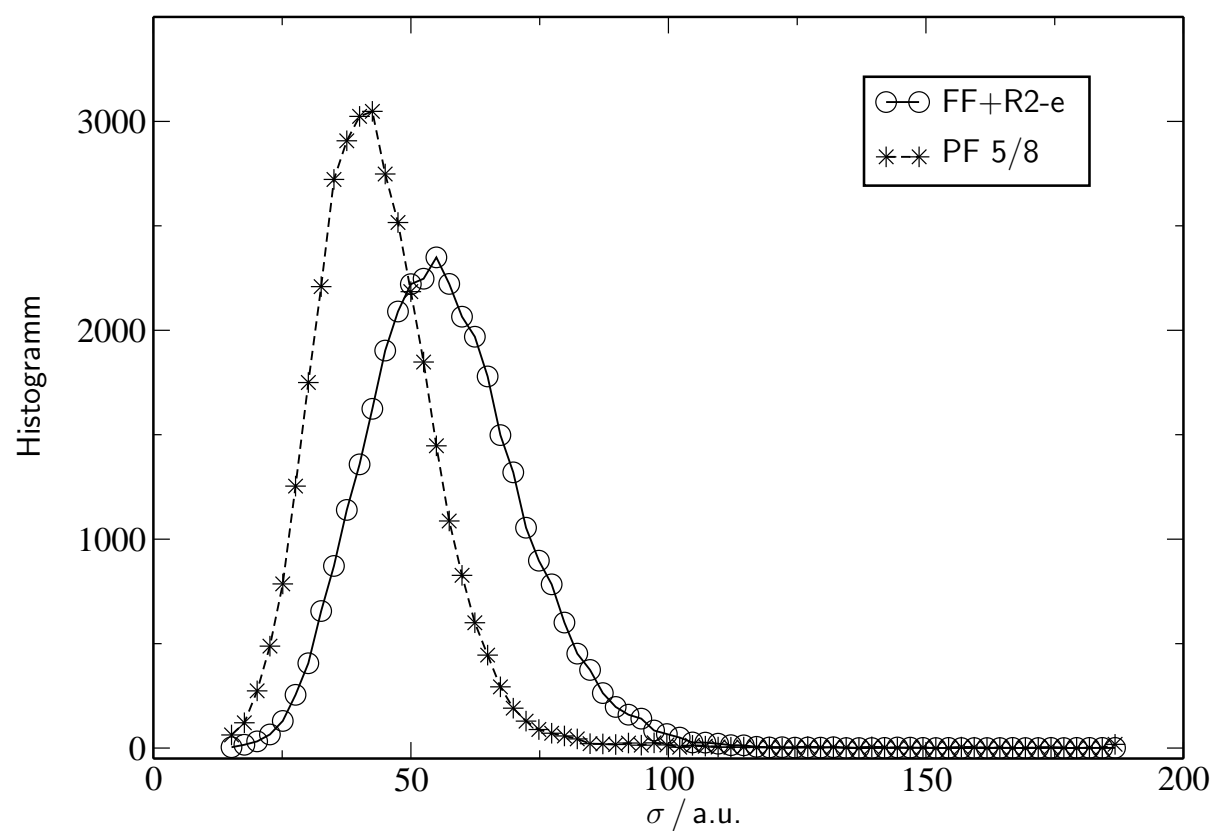

Abbildung 4.12: $\sigma$-Histogramm. Durch die Verschlechterung der Standardabweichung in Messungen mit FF+R2-e relativiert sich der theoretische Vorteil dieser Methode gegenüber der Referenzsequenz PF 5/8. Im Gegensatz zu konventionellen Methoden liefert die EnsembleMessung zur Bestimmung von $\sigma$ einen realistischeren Wert, der nicht durch die in POCSRekonstruktionen beobachtete Verfälschung des Rauschuntergrundes beeinträchtigt wird. 


\section{KAPITEL 5}

\section{Faserverfolgung}

\subsection{Konventionelle Techniken}

Die Aufklärung der Struktur neuronaler Netzwerke im menschlichen Gehirn ist grundlegend für das Verständnis seiner Funktionsweise. Angesichts der Relevanz dieser Fragestellung erscheint der gegenwärtige Wissensstand um die axonale Konnektivität noch bemerkenswert unvollständig und inkonsistent, zumal die moderne Neuroanatomie schon im neunzehnten Jahrhundert unter anderem durch die Arbeiten von Golgi und Ramón y Cajal als eigenes Wissenschaftsgebiet etabliert wurde. Im Laufe der Zeit entstanden viele Methoden für die Traktographie, die hier im weitesten Sinne als ein Oberbegriff für Ansätze zur Erlangung von Informationen über die Topologie langreichweitiger Nervenfasern dienen soll. Um die potentielle Bedeutung der Diffusionstensorbildgebung für diesen Forschungszweig einschätzen zu können, werden hier zunächst die Prinzipien herkömmlicher Ansätze skizziert.

Traktographie-Techniken teilen sich grob in direkte und indirekte Verfahren. Ohne explizite Lokalisierung von Axonverbünden ist es zum Beispiel anhand von bereits in ihrer Funktion kartierten Cortexarealen möglich, über Ausfallerscheinungen aufgrund von Läsionen oder Veränderungen in der weißen Hirnsubstanz, wie sie durch Tumore und neurodegenerative Erkrankungen hervorgerufen werden können, Rückschlüsse auf den Faserverlauf zu ziehen. Wegen fallbezogener und individueller Unterschiede muß dieser Ansatz recht induktiv bleiben. Er eignet sich nur bedingt für systematische Untersuchungen. Mit invasiveren Methoden gewonnene Erkenntnisse aus Tiermodellen sind in vielerlei Hinsicht oft nicht übertragbar. Ein weiteres Beispiel für ein indirektes Verfahren beruht ebenfalls auf einem NMR-Experiment. In der funktionellen Bildgebung [90], fMRI (functional Magnetic Resonance Imaging), wird über den Umweg metabolisch bedingter Änderungen der magnetischen Eigenschaften von Gewebe mit angeregten Neuronenpopulationen Hirnaktivität nachgewiesen. Korrelationen in diesen Aktivitätsmustern können auf direkte Verbindungen von separaten Arealen der grauen Substanz über ausgedehnte Axone hindeuten [91]. 
Die Methoden, mit denen man konventionell versucht, Nervenfaserbahnen direkt abzubilden, sind ausnahmslos invasiv und beruhen entweder auf in-vitro Untersuchungen oder auf am Menschen nicht anwendbaren Kontrastmittelverfahren. Im klassischen Hirnschnitt sind z. B. Axonverbünde durch Färbetechniken, die die Myelinschicht hervorheben, abbildbar [92, 93]. In Gewebe mit sich mischenden Nervenfaserpopulationen ist ihr Verlauf häufig nicht mehr zu eruieren. Hirnschnitte sind außerdem das Ausgangsmaterial für mikroskopische Untersuchungen mit linear polarisiertem Licht, dessen Ebene durch die Anisotropie des Gewebes charakteristisch gedreht wird, und das auf diese Weise die Identifikation von zusammengehörigen Strukturen erlaubt [94]. In beiden Fällen beeinträchtigt die Art der Präparation eine dreidimensionale Rekonstruktion. Von dieser Einschränkung nicht betroffen ist die Methode nach Klingler [95]. Ein formalinfixiertes Gehirn wird dazu in mehreren Zyklen tiefgefroren und wieder aufgetaut, wobei die Bildung von Eiskristallen der Separation von Faserbündeln Vorschub leistet. Diese lassen sich dann mit viel Umsicht und einem Spatel voneinander trennen. Kreuzende Axone und die Rückübertragung der Erkenntnisse auf anatomische Referenz-Koordinaten stellen auch bei dieser Methode ein Problem dar. Außerdem muß zur Präparation eines einzelnen Traktes das umliegende Gewebe zerstört werden.

Einige in-vivo Färbetechniken beruhen auf dem aktiven Transport eines Kontrastmittels durch die Nervenzellen, manchmal sogar über Synapsen hinweg, und lösen einige der angesprochenen Probleme. Ihre Anwendung am Menschen verbietet sich aber wegen der Invasivität und der toxischen Wirkung dieser sogenannten „Tracer“, z. B. radioaktive Substanzen oder Mangan, deren Anreicherung durch bildgebende Verfahren wie MRI räumlich aufgelöst dargestellt werden kann. Zu diesen Studien findet der Leser einen Überblick und Referenzen auf die einschlägige Literatur in der Sonderausgabe des Journal of Neuroscience Methods, Band 103 (2000). Kontrastmittelverfahren lassen sich am menschlichen Gehirn nur in post-mortem Studien durchführen, wobei man mangels Zellaktivität auf die Diffusion als Transportmechanismus zurückgreifen muß, was die Untersuchung auf sehr kurze Verbindungen beschränkt, sie äußerst langwierig und anfällig für Artefakte macht. Mit Färbetechniken ist es außerdem möglich, im Zuge von Neurodegeneration nach axonalen Schäden (Wallersche Degeneration) auftretende chemische Zerfallsprodukte zu markieren und so Faserverläufe zu visualisieren. Dieser Prozeß kann auch in mit entsprechend sensiblen Kontrasten ausgestatteten MR-Bildern beobachtet werden [96], so daß sich das Prinzip über die histologische Präparation hinaus auch auf in-vivo Untersuchungen ausweiten läßt. Wie schon angemerkt, ist ein Zugang über pathologische Einzelfälle schwierig zu systematisieren.

\subsection{DTI-Traktographie}

Durch die räumlich aufgelöste Messung und die Diagonalisierung des Diffusionstensors in gerichtetem Gewebe erhält man eine voxelweise Schätzung der Orientierung anisotroper Strukturen. Damit lassen sich unter gewissen Voraussetzungen Verläufe von Axonbündeln oder Muskelfasern dreidimensional rekonstruieren, indem man eine Startregion definiert und die beiden Äste des Traktes symmetrisch nach einer im einfachsten Fall MDD- 
basierten Propagationsvorschrift verfolgt [97]. Eine Unterscheidung zwischen afferenten und efferenten Neuronenbahnen, also die Bestimmung der Richtung der Erregungsleitung, ist mit diesem Prinzip allerdings nicht möglich.

Die bereits in Abschnitt 3.3 diskutierten Artefakte tragen zur Unsicherheit bei der Schätzung der Faserorientierung mit DTI bei. Verschiedene sogenannte probabilistische Traktographie-Strategien [98, 99] versuchen, diesem Umstand Rechnung zu tragen, und dabei zusätzlich die Konnektivität zu quantifizieren, indem sie aus den Meßdaten lokale Wahrscheinlichkeitsverteilungen der Hauptdiffusionsrichtung berechnen, um dann durch „sampling“ eine Vielzahl von potentiell zulässigen Pfaden zu generieren. Die Befürworter dieser Methode interpretieren die relative Häufigkeit der Anzahl der Verbindungen zweier Voxel bzw. ROIs als Vertrauensmaß, Verbindungsstärke oder gar als „Wahrscheinlichkeit" für die Verschaltung der beiden Regionen. Eine Bewertung dieses Ansatzes und die schwierige Frage nach der Quantifizierbarkeit von Traktographie-Ergebnissen sind nicht Gegenstand dieser Arbeit. Hier wird auf weitaus einfachere, deterministische Algorithmen [100] zur Rekonstruktion dreidimensionaler Faserverbindungen zurückgegriffen.

Faßt man eine Faser als eindimensionale Mannigfaltigkeit auf, deren Tangentialraum durch die Hauptdiffusionsrichtungen $\boldsymbol{\epsilon}_{1}(\mathbf{D}(\boldsymbol{r}))$ aufgespannt wird, läßt sich ihr durch die Weglänge $s$ parametrisierbarer Verlauf durch Lösung der Differentialgleichung

$$
\frac{\mathrm{d} \boldsymbol{r}(s)}{\mathrm{d} s}=\boldsymbol{\epsilon}_{1}(\mathbf{D}(\boldsymbol{r}(s)))
$$

ermitteln. Dazu stehen bekannte Algorithmen wie das Euler- oder das Runge-KuttaVerfahren bereit. Allerdings existiert nur eine verrauschte, räumlich diskrete Schätzung der Tangentialvektoren, weshalb die Exaktheit der Rekonstruktion durch die Voxelgröße und die Datenqualität eingeschränkt ist, und sie sich deshalb nicht beliebig über die Schrittweite einstellen läßt.

Eine dem Problem auf natürliche Weise angepaßte adaptive Schrittweite resultiert aus dem von Mori vorgeschlagenen FACT-Algorithmus [101] (Fiber Assignment by Continuous Tracking). Die Propagationsrichtung ist ebenfalls parallel zur Hauptdiffusionsrichtung, die so lange weiterverfolgt wird, bis der Pfad auf eine Voxelwand stößt, was eine Aktualisierung der Orientierung mit der Richtung des betretenen Bildelementes bewirkt. Bei der Implementation muß beachtet werden, daß Situationen auftreten können, in denen der neue Vektor wieder auf das eben verlassene Voxel weist, was eine Endlosschleife zur Folge hat. Sie kann vermieden werden, wenn man nach einer solchen Reflektion einen Aktualisierungsschritt in Bezug auf die Feststellung der Faserrichtung ausläßt.

Durch die Faserverfolgung enstehen neue Klassen von Artefakten. Zum einen schlagen sich systematische und statistische Fehler bei der Rohdatenakquisition in einer Unsicherheit für die geschätzten Richtungen nieder. Zum anderen bewirkt ein Verlassen des Axonbündels nicht notwendigerweise den Abbruch des Traktographie-Algorithmus'. Vielmehr kann es in diesem Fall zu einem Übersprung in benachbarte Bahnen kommen, wodurch falsch positive Verbindungen suggeriert werden.

Auf der Datenebene des Diffusionstensormodells sind außerdem Orientierungen in Voxeln mit mehreren, unter Umständen einander durchdringenden Axonpopulationen nicht 
auflösbar. Einfache Faserkreuzungen führen zu diskusförmigen Diffusionsellipsoiden, wobei der Eigenvektor $\boldsymbol{\epsilon}_{1}$ im Fall einer rechtwinkligen Begegnung prinzipiell beliebig in der Ebene orthogonal zu $\boldsymbol{\epsilon}_{3}$ gedreht sein kann. Solche Konstellationen machen sich in Form eines erhöhten $c_{\mathrm{p}}$-Wertes des Diffusionstensors (siehe Gleichung 3.11c) bemerkbar. Allerdings gilt der Umkehrschluß nicht, denn auch auf der Längenskala der Voxeldimensionen stark gekrümmte Bahnen haben unter Umständen diese Signatur. Andere singuläre Konfigurationen entstehen, wenn sich zwei gebogene Fasern ohne Durchdringung in einem Punkt berühren oder sich vereinigen bzw. aufspalten. Zumindest das Kreuzungsproblem läßt sich nicht durch eine Verringerung der Voxelgröße lösen.

\subsection{Regularisierung und singuläre Punkte}

Die mangelnde Robustheit der DTI-Traktographie hat neben den genannten Problemen auch einen Vorteil. Definiert man mehrere Startpunkte des gewählten Algorithmus' in einem Voxel, werden bei sich aufspaltenden Fasern auch mehrere Verzweigungspfade verfolgt. Die vollständige Darstellung des Traktes ist damit natürlich nicht garantiert, und in den berechneten Trajektorien lassen sich a priori korrekte Bahnen nicht von falsch positiven Verbindungen unterscheiden. Im Allgemeinen machen es die Rekonstruktionsartefakte unumgänglich, Start-, Abbruch- und Ausschlußkriterien auf der Basis von Annahmen oder gesicherten Informationen festzulegen. Weil man die Faserverfolgung auf DTI-Daten als inverses Problem auffassen kann, redet man in der Sprache dieses mathematischen Themenbereichs von Regularisierung. Naheliegend sind zum Beispiel die Einschränkung des Algorithmus' auf die weiße Hirnsubstanz durch die Definition einer unteren Anisotropieschranke, eines Graustufenbereichs auf einer anatomischen Referenzaufnahme oder die Voraussetzung einer begrenzten Faserelastizität bzw. minimalen Steifheit, d. h. der Winkel zwischen zwei aufeinanderfolgenden Rekonstruktionsvektoren unterliegt einer oberen Schranke, um die Stetigkeit des Pfades zu gewährleisten. Leider erweist sich, daß es wegen der unterschiedlichen Geometrien und Gewebstexturen keine Wertebereiche mit universeller Gültigkeit gibt, so daß die Parameter in einem gewissen Maß individuell an den darzustellenden Trakt anzupassen sind.

Ein nützlicher Ansatz, der allerdings ein noch größeres Maß an Vorwissen zur Auswahl vertrauenswürdiger Verbindungen voraussetzt, besteht in der Verwendung von MultiROI-Verfahren [97, 102]. Dazu definiert man neben den Startvoxeln, die im allgemeinen in einer Ebene orthogonal zur vermuteten Faserrichtung liegen sollten und deshalb sinnvollerweise auf den farbkodierten MDD-Karten ausgewählt werden, weitere Regionen, die entweder dazu dienen, durch sie passierende Pfade auszuschließen oder aber diese explizit zu selektieren. Auf diese Weise lassen sich viele Bahnen gezielt isolieren. Der Erkenntnisgewinn ist dagegen natürlich eingeschränkt, und Fehler bei der Definition der zusätzlichen ROIs können unter anderem unvollständige Darstellungen zeitigen.

Angesichts der Problematik kreuzender Fasern liegt es nahe, Regularisierungsmechanismen direkt in die Traktographie-Algorithmen einzubauen und auf der Ebene der Richtungsschätzung zusätzliche Annahmen oder Informationen einfließen zu lassen. Der Tensorlines-Algorithmus [103] versucht über eine an den Linearitätsparameter $c_{1}$ gekop- 
pelte, adaptive Richtungsberechnung, in einigen singulären Bereichen, also den im vorigen Abschnitt beschriebenen fehlerinduzierenden Geometrien, eine stabilere Pfadverfolgung zu gewährleisten. Dazu wird ein zusätzlicher Steifigkeitsterm eingebaut, der im $n$-ten Iterationsschritt eine gewichtete Summe aus vorheriger Propagationsrichtung $\boldsymbol{v}_{n-1}$ und demselbem Vektor allerdings durch die Diffusionstensormatrix gedreht darstellt

$$
\boldsymbol{v}_{n}:=c_{1} \boldsymbol{\epsilon}_{1}+\left(1-c_{1}\right)\left((1-w) \boldsymbol{v}_{n-1}+w \mathbf{D} \boldsymbol{v}_{n-1}\right),
$$

wobei $w \in[0,1]$ ein anzupassender konstanter Wichtungsfaktor ist. Weil die Faserrichtung in Regionen mit hohem $c_{\mathrm{p}}$ orthogonal zu $\epsilon_{3}$ nicht wohldefiniert ist, macht dieser Ansatz insofern Sinn, als daß dort durch die Matrixmultiplikation die Projektion des Ausgangsvektors auf die betroffene Ebene unverändert bleibt (zumindest ihre Orientierung), weil der Tensor diesen Teilraum invariant läßt. Im Gegensatz zu einfachen MDD-Strategien, macht man sich dabei direkt das volle Spektrum des Diffusionstensors zunutze. Bis dato wurde das Tensorlines-Prinzip an numerischen Phantomen getestet und seine Anwendbarkeit am Beispiel des Corpus callosums und seiner Faserkreuzungen zumindest plausibel gemacht, aber die Validität der gefundenen Trajektorien nicht explizit verifiziert. Ein Kritikpunkt an dem Algorithmus ist, daß er in einigen Bereichen die Krümmung der Faserbahn nicht korrekt nachvollzieht.

Über die Verhältnisse der skalaren Hauptdiffusivitäten hinaus scheinen sich auch abgesehen von $\boldsymbol{\epsilon}_{1}$ zusätzliche sinnvolle Informationen in der geometrischen Eigenstruktur von $\mathbf{D}$ zu verbergen, die möglicherweise zur Regularisierung ausgenutzt werden können. So beobachtet man bei einigen Nervenfasern einen schichtartigen Aufbau, der sich in der Ausrichtung der zweiten und dritten Eigenvektoren niederschlagen sollte. Tatsächlich entdeckt man in entsprechend farbkodierten Abbildungen (Abbildung 5.1) von $\boldsymbol{\epsilon}_{2}$ (und notwendigerweise ebenso bei $\boldsymbol{\epsilon}_{3}$ ) auch dort Regelmäßigkeiten in der Orientierung [104].

\subsection{DeffCoN}

Zur quantitativen und qualitativen Erforschung der Diffusionseigenschaften des menschlichen Gehirns und seiner dreidimensionalen neuronalen Konnektivität wurde im Rahmen der vorliegenden Arbeit ein Computerprogramm, DeffCoN (effective Diffusion Coefficient Navigator), erstellt, das einen eingeschränkt interaktiven Zugang zu den DTI-Daten bietet. In Abbildung 5.2 ist das Bildschirmphoto einer Auswertesitzung zu sehen.

DeffCoN wurde in $\mathrm{C}++$ unter dem Betriebssystem Linux programmiert. Die Realisierung stützte sich dabei maßgeblich auf Software-Bibliotheken für wissenschaftliches Rechnen (GSL [105], BLAS/ATLAS [106, 107], LAPACK [108]), die unter der GPL (GNU Public License) verfügbare Version von Qt [109] zur Erstellung einer graphischen Benutzeroberfläche und die auf Visualisierung und graphische Datenverarbeitung abzielenden Bibliotheken VTK [110] und ITK [111]. 

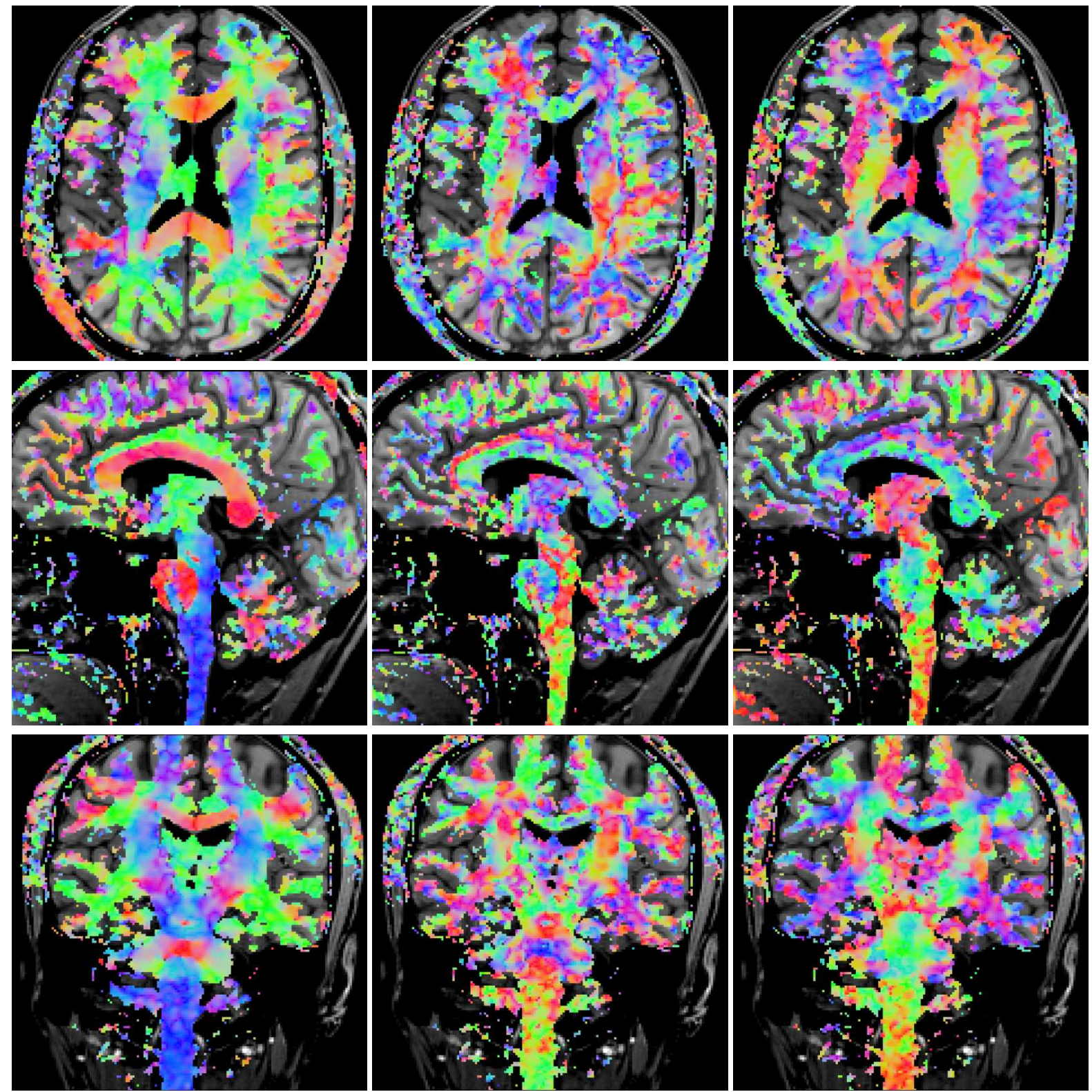

Abbildung 5.1: Farbkodierte Karten der Eigenvektor-Orientierungen. Die Reihen entsprechen von oben nach unten transversaler, sagittaler und coronaler Schnittführung. In Spalten angeordnet sind die Darstellungen von (links) $\boldsymbol{\epsilon}_{1}$ bzw. MDD, (Mitte) $\boldsymbol{\epsilon}_{2}$ und (rechts) $\epsilon_{3}$. 


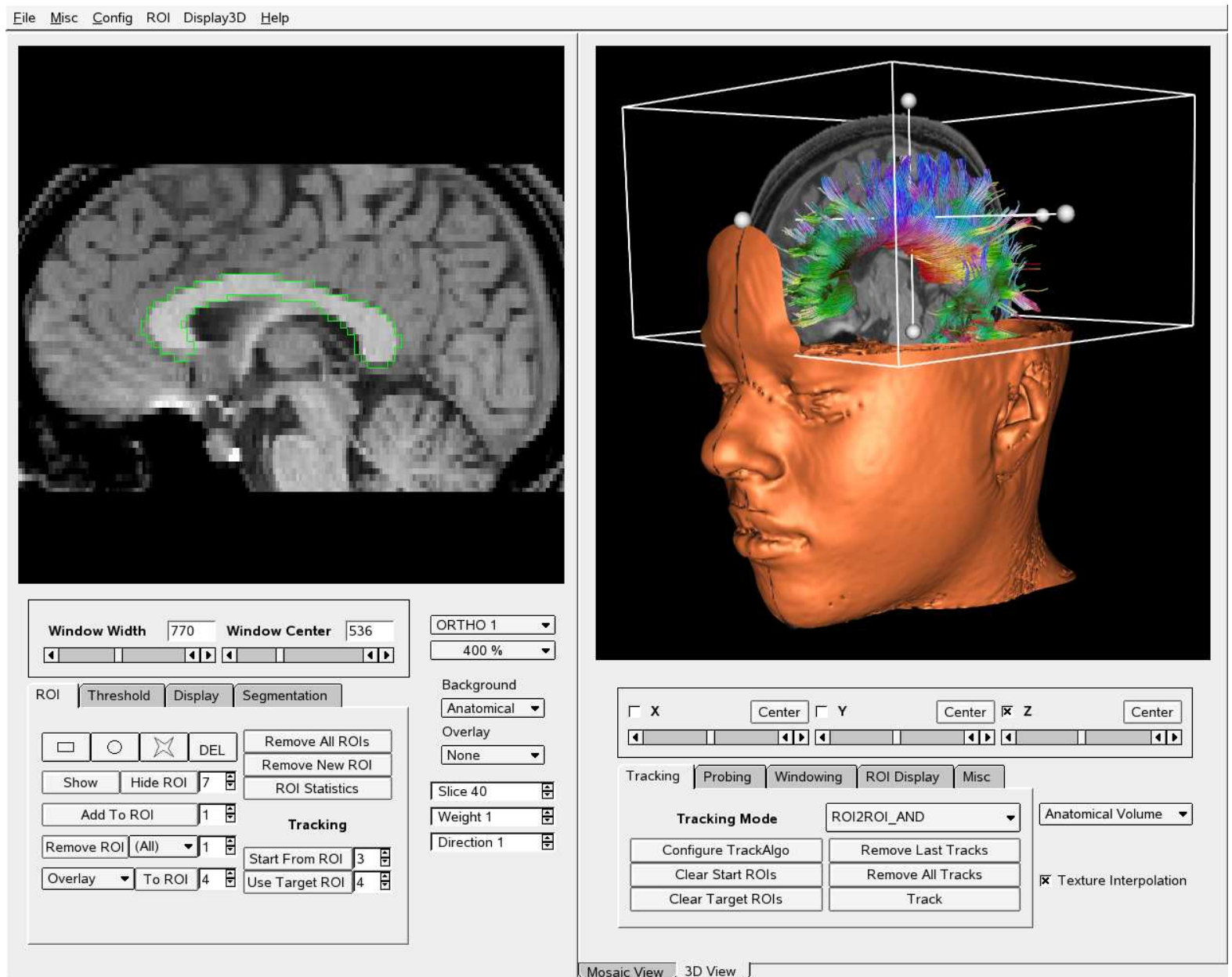

Abbildung 5.2: DeffCoN Bildschirmphoto. Darstellung von zwei- und dreidimensionalen Analysemöglichkeiten. Die auf einer MDD-Karte definierte und auf die Anatomie-Referenz übertragene grüne ROI des Corpus callosums (links), belegt die geometrische Korrektheit einer DW-SSSTEAM-Aufnahme.

\subsubsection{Datenverarbeitung}

Nach dem Einlesen der Schichtbilder, die im DICOM-Format vorliegen müssen, berechnet DeffCoN den Diffusionstensor voxelweise entweder durch lineare Regression oder durch einen nichtlinearen Fit mit Hilfe des Levenberg-Marquardt-Algorithmus', der zwar in der weißen Hirnsubstanz geringfügig bessere Ergebnisse liefert [89], aber wegen des weitaus größeren Zeitaufwandes nur selten zum Einsatz kommt. Optional läßt sich bei Vorliegen einer entsprechenden Messung vorher eine Neeman-Mittelung (siehe Abschnitt 3.3.1) durchführen. Häufig erfordert das geringe SNR von DW-SSSTEAM-Daten auch deren räumliche Glättung, wofür ein dreidimensionaler Gauß-Filter oder kantenerhaltende Algorithmen aus der Softwarebibliothek ITK in einem weiteren anwählbaren Vorverarbeitungsschritt eingesetzt werden können. Das Diffusionsschema, d.h. die Anzahl und Orientierung der Diffusionsrichtungen und -wichtungen, ist dabei weitgehend beliebig 
und wird DeffCoN in Form einer Parameter-Datei übergeben. Außer dem Tensor errechnet das Programm die in Abschnitt 3.1 vorgestellten Karten, z. B. die fraktionelle Anisotropie, und speichert diese Daten sowie die durch Diagonalisierung gewonnenen Hauptachsen der Diffusionsellipsoide. Es besteht die Möglichkeit in ihrer Aufnahmeorientierung frei wählbare anatomische Referenzaufnahmen hinzuzuladen, die dann anhand der im DICOM-Format beinhalteten geometrischen Informationen relativ zu den Diffusionsbildern räumlich konsistent reformatiert und transformiert werden. Die Datenverarbeitung dauert auf aktuellen handelsüblichen Heim-Rechnern im einfachsten Fall sogar bei umfangreichen Datensätzen von 80 Schichten nur wenige Minuten.

\subsubsection{Programm-Funktionalität}

Dem Benutzer stehen in einem 2D-Teilbereich von DeffCoN neben der Visualisierung von Originaldaten und berechneten Karten in drei orthogonalen Ansichten auch quantitative Histogramm- und ROI-Analysemöglichkeiten (Region Of Interest) zur Verfügung. Unterstützend lassen sich Schwellenwerte definieren, um die Darstellung von dem jeweiligem Hintergrundbild überlagerten Abbildungen der Hauptdiffusionsrichtung zu steuern. Die Schnittstelle des Programms zu einem Segmentierungsalgorithmus aus der ITK-Bibliothek hilft bei der Auswahl von Landmarken. Jede ROI kann dem 3D-Modul übergeben werden, um sie als Start- oder Zielregion von Faserverfolgungsalgorithmen zu deklarieren. In der 3D-Ansicht dient die verstellbare Schnittebenenansicht einer anatomischen Referenz (im einfachsten Fall die ungewichteten Diffusionsbilder) zur räumlichen Orientierung, zu der auch die dreidimensionale Darstellbarkeit der ROIs noch einmal beiträgt. Die Hauptfunktionalität dieses Programmteils besteht in der Faserverfolgung, für die verschiedene parametrisierbare Algorithmen zur Verfügung stehen (Euler, RungeKutta, FACT, Tensorlines). Die berechneten, durch Röhren dargestellten Pfade lassen sich individuell ansprechen und uniform oder bunt einfärben, entweder gemäß der MDDKodierung oder durch die über eine beliebig definierbare Farbtabelle abgebildeten, bei jeder Algorithmusiteration ermittelten FA- oder $c_{\mathrm{p}}$-Werte.

\subsubsection{Typisches Vorgehen}

Für die in dieser Arbeit routinemäßig angewandte Vorgehensweise zur Faserverfolgung werden die Rohdaten nach der Neeman-Mittelung linear auf eine Voxelgröße von $1 \times 1 \times$ $1 \mathrm{~mm}^{3}$ interpoliert. Die anschließende Anwendung eines dreidimensionalen Gauß-Filters mit einer Breite von $\sigma=1 \mathrm{~mm}$ dient zur Rauschunterdrückung.

Unter Verwendung von anatomischen Atlanten $[112,113]$ lassen sich z. B anhand koregistrierter, $T_{1}$-gewichteter Bilder besonders aber mit Hilfe der MDD-Karten im Idealfall orthogonale Querschnitte bekannter Faserbahnen identifizieren und als Startregionen für die Traktographie definieren.

Als Standard-Algorithmus kommt FACT zum Einsatz, wobei der Diffusionstensor an den Iterationspunkten des berechneten Pfades linear interpoliert und dann zur Bestimmung der MDD diagonalisiert wird. In jedem Schritt kontrolliert DeffCoN die Regularisierungs- bzw. Abbruchbedingungen. Je nach Eigenschaften des Traktes und der Topologie 
der angrenzenden Bahnen variieren die empirisch bestimmten Werte für die untere FASchwelle von 0.06 bis 0.1 und für den maximale Ablenkungswinkel von ca. $20^{\circ}$ bis $40^{\circ}$. In jedem Startvoxel stellen der Mittelpunkt und die Zentren des in acht gleiche Quader geteilten Volumenelements Anfangspunkte der Traktographie dar.

\subsection{Ergebnisse}

Mit DTI-Faserverfolgung konnten in DeffCoN zahlreiche dokumentierte Nervenbündel des menschlichen Gehirns abgebildet werden. Dabei machen sich allerdings auch die bereits diskutierten Schwächen des Verfahrens in Regionen mit dicht gepackten oder kreuzenden Bahnen bemerkbar. Die Exaktheit der Rekonstruktion läßt sich mangels Verifikationsmöglichkeit schwer ermessen, da oft nur grobe oder unvollständige Referenzen zur Verfügung stehen. Erschwert wird die Situation durch die teilweise uneinheitliche oder sogar widersprüchliche Darstellung in der Literatur. Die Diskussion der Resultate muß also auf einem deskriptiven und manchmal hypothetischen Niveau erfolgen. Sie orientiert sich hier am Maßstab anerkannter neuroanatomischer Fachbücher [113, 112, 114]. Die Datengrundlage der Ergebnisse bilden ohne besondere Systematik DW-SSSTEAM-Aufnahmen mit der Partial Fourier Referenzimplementation und mit dem PPI-beschleunigten Typ ( $R=2,25$ externe Referenzlinien) aus Kapitel 4 (Sequenzparameter: siehe Anhang A). Obwohl hier kein Sequenzvergleich auf der Basis von Traktographie beabsichtigt ist, sei darauf hingewiesen, daß insbesondere die im folgenden gezeigten Assoziationsbahnen, die zum Hirnstamm gehörigen Trajektorien und wichtige Teile des visuellen Systems Rekonstruktionen aus Messungen mit der neuen Variante FF+R2-e entstammen.

Mit Blick auf die Auflösung der NMR-Daten ist festzustellen, daß nur relativ breite Axonbündel zuverlässig abbildbar sind, wogegen z. B. bei der Verfolgung der Commissura anterior mit ihrem Durchmesser von in einigen Bereichen nur ca. ein bis zwei Aufnahmepixeln auch aufgrund von benachbarten, orthogonal verlaufenden Fasern des Fornix nicht immer überzeugende Ergebnisse erzielt werden können. Im Hirnstamm, im Thalamus und in hypothalamischen Regionen erweist sich die mit der DW-SSSTEAM-Sequenz zur Zeit erreichbare Auflösung als zu grob, um den diffizilen Verhältnissen gerecht zu werden. Zumeist sind die Namen der rekonstruierten Bahnen nur Oberbegriffe feinerer Substrukturen. Interessanterweise konnten mit den zur Verfügung stehenden Daten keine reinen Hirnnerven des peripheren Nervensystems (PNS) dargestellt werden, was einerseits an ihrer verzweigten Struktur und andererseits an zu geringen Signalintensitäten liegen mag. Daß die Diffusionstensor-Technik nicht selektiv sensibel auf Neuronenbündel, sondern allgemein anisotrop texturiertes Gewebe reagiert, zeigt in diesem Kapitel das Beispiel der Augenmuskulatur.

Topologisch unterscheidet man zwischen Kommissurbahnen, die die beiden Hemisphären des Gehirns miteinander verbinden, Projektionsbahnen als Träger des Informationsflusses von subkortikalen Kernen zur grauen Substanz der Hirnoberfläche (Cortex) und Assoziationsbahnen, welche Verknüpfungen von Cortex-Regionen innerhalb oder zwischen einzelnen Hirnlappen (Lobus frontalis, L. parietalis, L. occipitalis und L. temporalis) herstellen. Bei der folgenden Zusammenstellung wurden die Nervenfasern, gegebenen- 
falls von dieser Klassifikation abweichend, teilweise nach ihrer geometrischen Anordnung gruppiert, teilweise nach ihrer funktionellen Zusammengehörigkeit. So ist z. B. das Cingulum als Assoziationsfaser ein wichtiger Bestandteil des limbischen Systems, in das es hier eingeordnet wird. Gleichzeitig ist die Commissura anterior eine Kommissurbahn, aber ihre Lage relativ zum Fornix macht es sinnvoll, die beiden Bahnen gemeinsam zu diskutieren. Für die anatomischen Konventionen im Hinblick auf Richtungs- und Bereichsbezeichnungen sei der Leser auf [114, Kapitel 4] verwiesen.

Die meisten der hier illustrierten Faserverbindungen sind bereits von anderen Gruppen mit DTI-Traktographie nachvollzogen worden $[102,115,116]$. Dank der unverzerrten Abbildung der Anatomie durch die DW-SSSTEAM-Sequenz war es in dieser Arbeit zusätzlich möglich, den Nervus opticus mit der ihn umschließenden Augenmuskulatur darzustellen. Aus dem gleichen Grund gelang es auch, eine qualitativ hochwertige und detailreiche Kartierung des Hirnstamms vorzunehmen. Dessen Bahnen hatten zuvor mit DTI explizit nur Stieltjes et al. [117] mithilfe einer segmentierten (d. h. „multi-shot“) DTIEPI-Sequenz vermessen, wobei die Ergebnisse trotz der angepaßten Akquisitionstechnik stark von Geometrieartefakten beeinträchtigt sind. Darüberhinaus wird in diesem Kapitel eine natürliche traktographiebasierte Segmentierung des vorderen Abschnittes der Capsula interna (Crus anterior) vorgestellt. Bei der Diskussion liegt der Schwerpunkt nicht auf der funktionellen Bedeutung einzelner Axonverbünde, die sowieso in weiten Teilen nicht sonderlich gut bekannt ist, sondern auf der Exaktheit der Darstellung und auf möglichen Artefakten. Obwohl eine aus physikalischer Sicht befriedigende Lösung des Problems komplizierter Gewebsgeometrien, respektive Faserkreuzungen, am Diffusionsmodell ansetzen muß, werden in diesem Abschnitt zwecks Vollständigkeit an zwei Beispielen die Vor- und Nachteile des Tensorlines-Algorithmus erläutert. Dabei dient Gleichung (5.2) als Vorschrift für die Propagationsrichtung während der entsprechende Teil des FACT-Algorithmus' die Schrittweite vorgibt.

\subsubsection{Corpus callosum}

Das Corpus callosum (CC), auch als Balken bezeichnet, stellt die ausgedehnteste Kommissurbahn und einen der größten logischen Faserverbünde des menschlichen Gehirns überhaupt dar. Auf einem medialen sagittalen Schnitt werden die vorderen Teile als Rostrum und Genu, der mittlere als Truncus und der hintere als Splenium corporis callosi bezeichnet, die sich zu einem nach unten offenen Bogen formen. Im mittleren bis hinteren Bereich kann es ventral zur Berührung mit Teilbahnen des Fornix (FX) kommen, die ebenfalls interhemisphärisch verlaufen und in der Commissura fornicis ähnlich orientierte Strukturen bilden, was dem Überspringen von Trajektorien in die jeweils benachbarten Bündel Vorschub leistet.

Die nächstgrößere Faserstruktur auf der dorsalen Seite ist das Cingulum (CG), dessen Axone medial größtenteils orthogonal zur Ausrichtung der darunterliegenden Kommissurbahnen verlaufen, und das dabei immer wieder Axonbündel in die Windungen des Gyrus Cinguli schickt. Es umschließt den Balken halbkreisförmig. Besonders im Genu und im Splenium ist dabei der Abstand meist äußerst gering, so daß ebenfalls mit TraktographieArtefakten zu rechnen ist. 
Im hinteren Abschnitt beinhaltet das Corpus callosum unter anderem nicht besonders gut dokumentierte Faserbündel, die über das untere Horn der lateralen Ventrikel in einer dünnen Schicht, dem Tapetum, zu den Temporallappen laufen. Hier besteht wegen der räumlichen Nähe und möglicherweise auch wegen Faserkreuzungen die Gefahr des Überspringens in den Fasciculus occipitofrontalis (FOF). Häufig ziehen einige dieser dann wahrscheinlich eher den Fibrae occipito- bzw. parietopontinae (FOPP) zuzuordnenden Trajektorien auch in den Hirnstamm. Bei der Faserverfolgung des Corpus callosums besteht das Problem, daß sich die Krümmung der weißen Substanz im medialen Bereich mit der Ventrikelgröße ändert. Eine Zunahme der Ausdehnung dieser Liquorkammern durch Hirnatrophie oder -resektionen führt dann zu einer Abflachung der Bahnen, so daß die Trajektorien in Extremfällen alle nach unten in die Capsula interna wechseln und das Resultat dem umgestülpten Original entspricht.

Im Kontrast zu den in Abbildung 5.3 dargestellten Ergebnissen, die eine in weiten Teilen der Radiatio corporis callosi, dem Teilstück vor der Einmündung in den Cortex, streng nach oben ausgerichtete Struktur suggerieren, sind in der Literatur oft auch stärker lateral verlaufende Faserstränge beschrieben (siehe z. B. [112, S.395],[113, S.42]). Man findet zwar recht häufig im frontalen Bereich nahezu waagrechte Trajektorien (siehe dazu Abbildung 5.4), im allgemeinen müßten die Bahnen des Corpus callosums aber, um der zitierten Darstellung zu entsprechen, sowohl Fasern der Capsula interna bzw. Corona radiata als auch des Fasciculus longitudinalis superior (FLS) kreuzen. Die erhöhten $c_{\mathrm{p}}$-Werte in den betreffenden Regionen lassen diese Möglichkeit tatsächlich plausibel erscheinen. Neben Indizien aus hochwinkelaufgelösten Diffusionsmessungen [118] sprechen auch die häufig auftretenden, wegen Verletzung von Steifigkeitsannahmen abbrechenden Trajektorien im ventralen Bereich des Truncus corporis callosi für diese Vermutung, die ansonsten bei aufgeweichten Bedingungen nach unten ziehen, dabei aber wohl in die Fasern der Capsula interna überspringen.

Um die Anwendbarkeit von zusätzlich regularisierter Traktographie zur Behebung dieses Problems zu illustrieren, sind in Abbildung 5.4 die Ergebnisse von FACT und Tensorlines (Abschnitt 5.3) einander zum Vergleich gegenübergestellt. Der Parameter $w$ aus der Propagationsregel (5.2) wurde auf 0,2 gesetzt, wie von den Urhebern vorgeschlagen. Gegenüber FACT zeigen die Trajektorien des Tensorlines-Algorithmus eine stärkere Zerfaserung der Bahnen, die nun im oberen Hirnbereich wie gewünscht auch laterale Cortex-Areale miteinander verbinden. Sie erscheinen bei dem gewählten Parametersatz recht steif, was zur Folge hat, daß die im Referenzbild zu beobachtende, ausgeprägt mediale Orientierung der inneren Fasern aufgrund der starken Krümmungen nicht mehr nachvollzogen werden kann. Dagegen vermeidet Tensorlines Projektionen in den Hirnstamm, und die Häufigkeit von Übersprüngen in den Fasciculus occipitofrontalis (inferior) ist reduziert.

Die Aufklärung der genauen Topographie des Corpus callosums ist Gegenstand aktueller Forschung. Inbesondere ist man an einer Parzellierung eines sagittalen Mittelschnitts nach Faserverlauf interessiert, also eine Klassifizierung nach den jeweils über bestimmte Regionen verbundenen funktionellen Zentren. Erste Schritte in diese Richtung mit Hilfe von DTI-Traktographie wurden von Huang et al. [119] unternommen. 

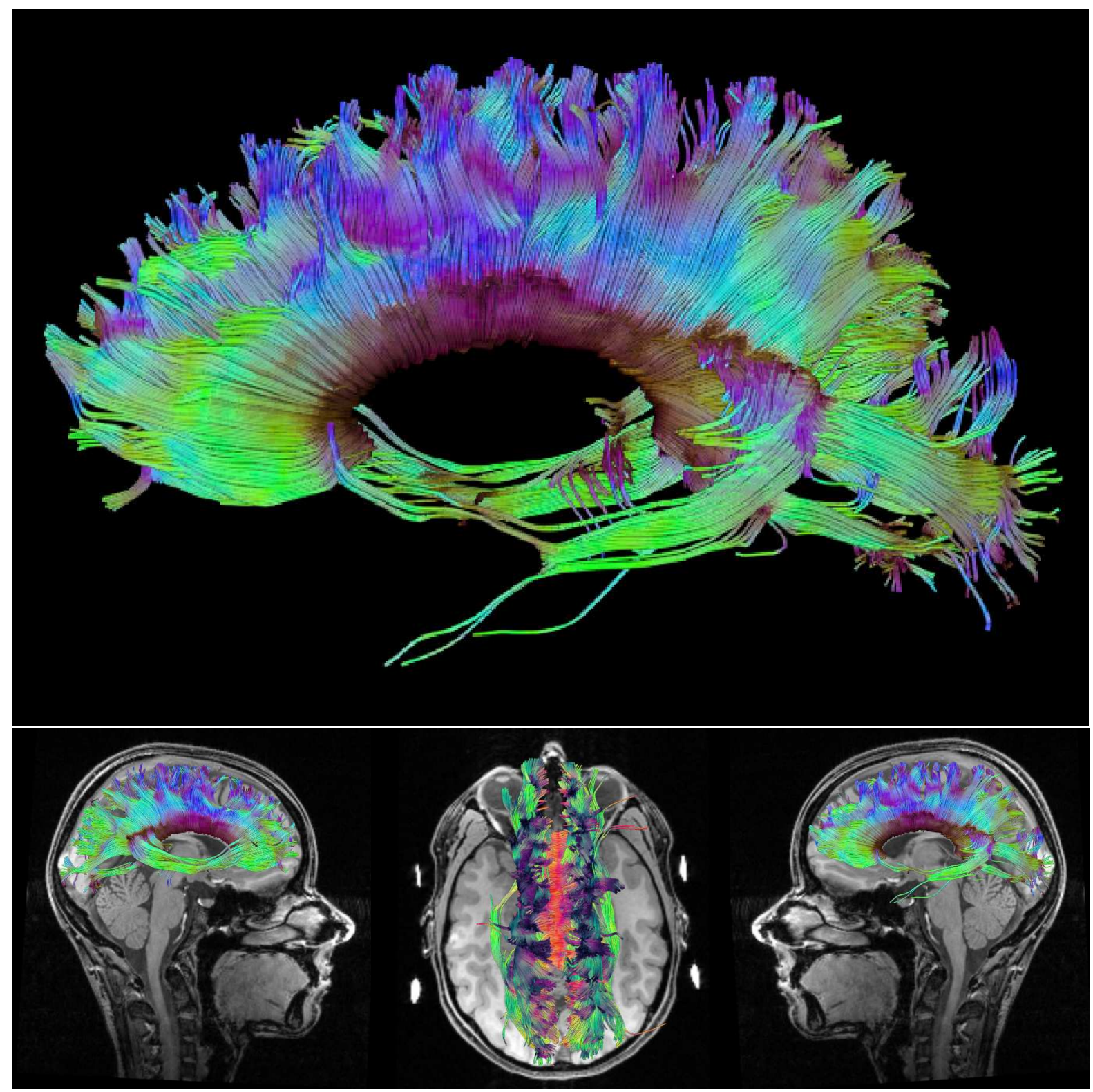

Abbildung 5.3: Corpus callosum. Rekonstruktion der Fasern des Balkens mit dem FACTAlgorithmus. 

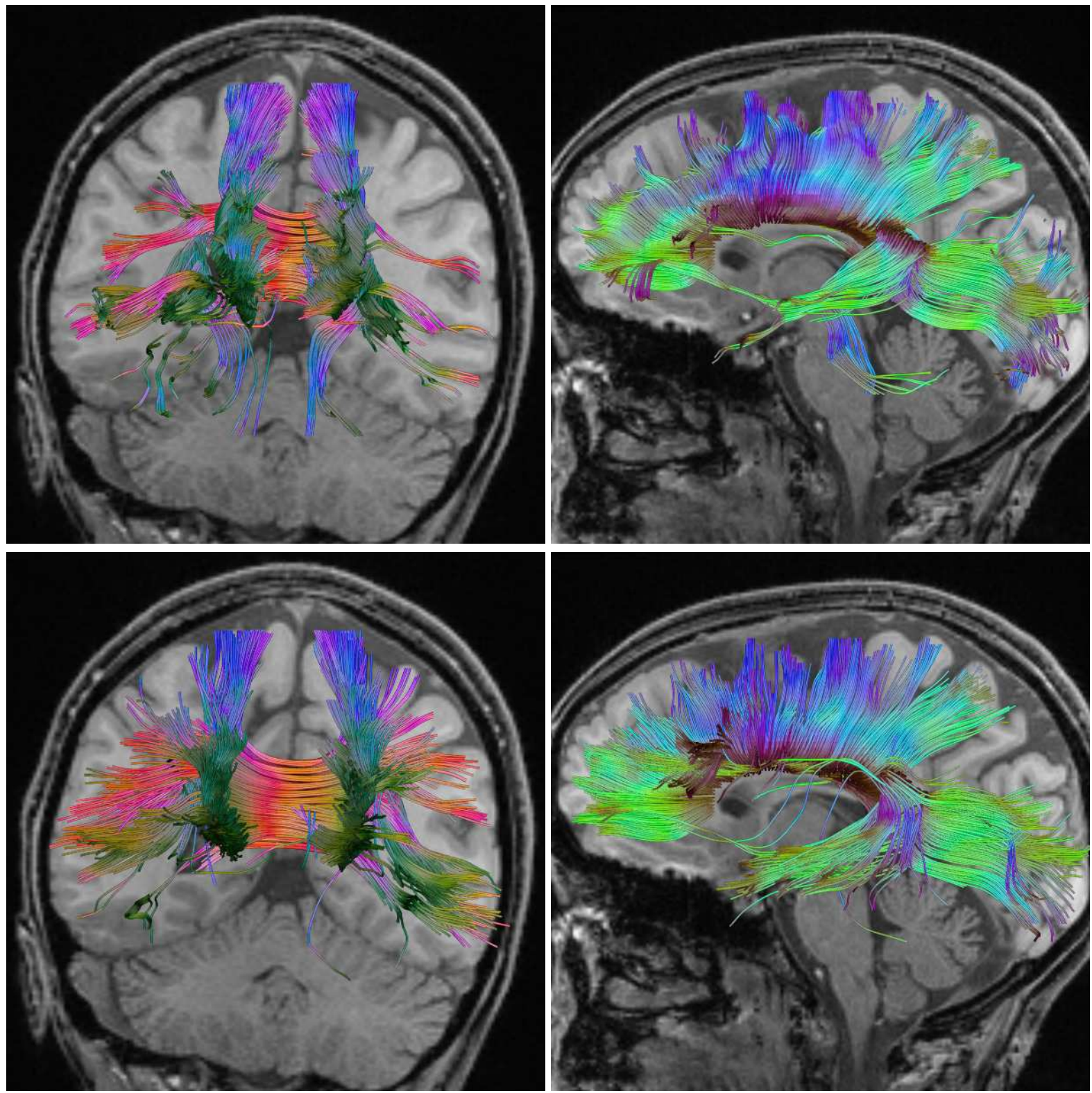

Abbildung 5.4: Laterale Verbindungen des Corpus callosums. Vergleich von Tensorlines-Trajektorien (untere Reihe) mit FACT-Resultaten (oben). Die Rekonstruktion beruht auf einem anderen Datensatz als Abbildung 5.3. 


\subsubsection{Limbisches System}

Das limbische System spielt eine Rolle bei der Verarbeitung von Emotionen. Die neuronale Konnektivität der assoziierten Hirnregionen wird von den Faserbahnen des Fornix (FX) und des Cingulums (CG) hergestellt, die sich als seine Hauptbestandteile zu dem sogenannten Papez-Kreis zusammenschließen.

Das Cingulum beinhaltet sowohl lang- als auch kurzreichweitige Verbindungen des Gyrus cinguli. Sein Verlauf wurde schon im vorangegangenen Abschnitt in Teilen umrissen. Für eine vollständige Beschreibung sind hier noch die sich hinter der kaudalen Grenze des Corpus callosums anschließenden Temporallappenprojektionen zu erwähnen. Technisch gesehen benötigt man zur Darstellung des Cingulums mit DTI-Traktographie typischerweise beidseitig jeweils vier Start-ROIs, deren Definition für die mittleren Teile in coronaler Ansicht, für die vorderen, hinteren und temporalen Abschnitte am besten auf transversal orientierten MDD-Farbdarstellungen erfolgt. Neben den bereits angesprochenen Problemen in der Nähe von Genu und Splenium corporis callosi treten auch im mittleren Abschnitt des Cingulums teilweise kommissurale Trajektorien (nicht dargestellt) auf, wobei einige ungeklärte Anteile nicht durch den Balken ziehen. Ansonsten entspricht die DTI-Rekonstruktion dieses Traktes in befriedigender Übereinstimmung der bekannten Literatur.

Die Axone des Fornix umschließen die Commissura anterior (CA) in einem prä- und postkommissuralen Teil. In zwei medial symmetrisch vom Corpus mammillare in Richtung des Truncus corporis callosi aufsteigenden Ästen (Columnae fornicis) krümmen sich die eng beieinanderliegenden postkommissuralen Hauptbahnen zu einem Bogen (Corpus fornicis) unter dem Corpus callosum und über dem Thalamus und laufen ab dem Crus fornicis lateral getrennt zu den Hippocampi in den Temporallappen der beiden Hemisphären, wo sie in den als Fimbriae bekannten Faserbündeln aufgehen.

Die ROI-Definition zur DTI-Faserverfolgung des Fornix (und der in dem gewählten Bereich ununterscheidbaren Stria terminalis) erfolgt typischerweise in einer koronalen Schicht am Crus fornicis. Wegen der in der Nähe ähnlich strukturierten ThalamusProjektionen sollte eine anatomische Referenzabbildung hinzugezogen werden. Selbst dann kommt es oft zu Übersprungartefakten zwischen parallel verlaufenden Bündeln. In manchen Fällen knicken auch einige Trajektorien, die unter Umständen mit der Stria medullaris thalamis in Beziehung stehen, im Bereich der Columnae fornicis kaudal in den Thalamus ab, für dessen vorderen Kern (Nucleus anterior thalami) unter anderem Anbindungen über den Fornix dokumentiert sind [113, S.195 u. 213]. Abbildung 5.5 zeigt im Widerspruch zur Literatur, daß die FACT-Trajektorien des Fornix nicht, wie eigentlich zu erwarten, postkommissural zum Corpus mammillare führen, sondern weiter frontal in den Nervus opticus (NO) abdriften. Interessanterweise beobachtet man häufig beim Einstellen der Abbruchkriterien, daß sich eine der beiden Temporallappenverbindungen des Fornix durch merklich niedrigere FA-Werte als die andere auszeichnet.

Die nicht zum limbischen System zählende Commissura anterior (CA) dient nach gegenwärtigem Wissensstand hauptsächlich dem Informationsaustausch zwischen den unteren Bereichen der beiden Temporallappen. Sie besteht aus neopallialen, amygdaloiden und intertuberkularen Anteilen [112, Kap. 7]), die vermutlich auch alle in der koronalen 
Ansicht in Abbildung 5.5 respektive von außen nach innen zu sehen sind (teilweise nur einseitig). Die Tensorlines-Rekonstruktion (Abbildung 5.6) zeigt außerdem in Ansätzen die als interbulbare Komponente der Commissura anterior bekannte Hemisphärenverbindung des Frontallappens zwischen den Bulbi olfactorii, die zum Riechzentrum gehören. Darüberhinaus gibt es auch Hinweise auf schwierig zu verfolgende kortikale Zielgebiete im unteren Okzipitallappen. Die DTI-Resultate mit FACT legen (hier nur auf einer Seite) manchmal zumindest kaudal verlaufende Fasern nahe, die Trajektorien liegen allerdings zu hoch, um mit den Ergebnissen von Di Virgilio et al. [120] als konsistent betrachtet werden zu können. Dagegen scheint der Tensorlines-Algorithmus auf dem Datensatz von Abbildung 5.6 dieser Beschreibung entsprechende Bahnen (neben einigen falsch positiven durch das Corpus callosum) zu detektieren. Seine Steifigkeitsanteile wirken sich offensichtlich auch vorteilhaft auf die Darstellbarkeit der Temporallappenkonnektivität aus.

Der kleine Durchmesser der Commissura anterior und die Nähe zu den Bahnen des Fornix provozieren das Auftreten von falsch positiven Verbindungen zu dem jeweils anderen Trakt. Allerdings werden in [113, S.191] auch Komponenten der Stria terminalis erwähnt, die in die Commissura anterior eintreten.

\subsubsection{Assoziationsbahnen}

Viele der langreichweitigen Assoziationsbahnen des menschlichen Gehirns sind wegen ihres großen Querschnitts mit DTI-Faserverfolgung auf grober Skala sehr gut darstellbar (Abbildungen 5.7 und 5.8). Neben den in diesem Abschnitt vorgestellten Axonverbünden gehört auch das schon beschriebene Cingulum (CG) in diese Klasse. Kurzreichweitige Assoziationsbahnen werden wegen ihrer Form auch U-Fasern genannt und verknüpfen einzelne Gyri/Hirnwindungen miteinander. Ein kleiner Anteil ihres Bestandes ist entlang der hier explizit abgebildeten großskaligen Trakte zu beobachten.

Der Fasciculus uncinatus (FU) ist ein hakenförmiges Faserbündel zur Verbindung der grauen Substanz des unteren Temporallappens mit dem Frontalbereich. Sein Verlauf demonstriert, daß die DTI-Traktographie auch stark gekrümmte Strukturen erfaßt. Bei der Definition der Startregion, typischerweise in einer koronalen Schicht im oberen, vorderen Bereich des Temporallappens, kommt es nahezu unweigerlich dazu, daß auch zum Fasciculus longitudinalis inferior (FLI) gehörige weiße Substanz markiert wird, der dort parallel, aber weiter ventral verläuft und dann in den okzipitalen Teil des Gehirns zieht. Bei beiden Axonbündeln ist ihre Nähe zum medial benachbarten (unteren) Fasciculus occipitofrontalis (FOF) zu beachten, dessen Verlauf vom Namen her nahezu selbsterklärend ist. Markant erscheint die Verengung seiner kaudal weit aufgefächerten Fasern in der Capsula externa in dem Bereich, ab dem er parallel zum Fasciculus uncinatus verläuft, sowie die anschließende erneute Aufweitung. Bei unvorsichtiger Wahl der Startregion erhält man viele, sehr wahrscheinlich falsch positive Verbindungen durch das Corpus callosum via Tapetum. Es empfiehlt sich deshalb, einen ROI-zu-ROI-Strategie zu verfolgen und die Startpunkte kaudal hinter das Tapetum und die Zielregion an die schmalste Stelle des vermuteten Verlaufs zu legen. Im Gegensatz zu fast allen anderen Assoziationsfasern ist das multi-ROI-Verfahren beim Fasciculus occipitofrontalis (inferior) nicht in dem Sin- 


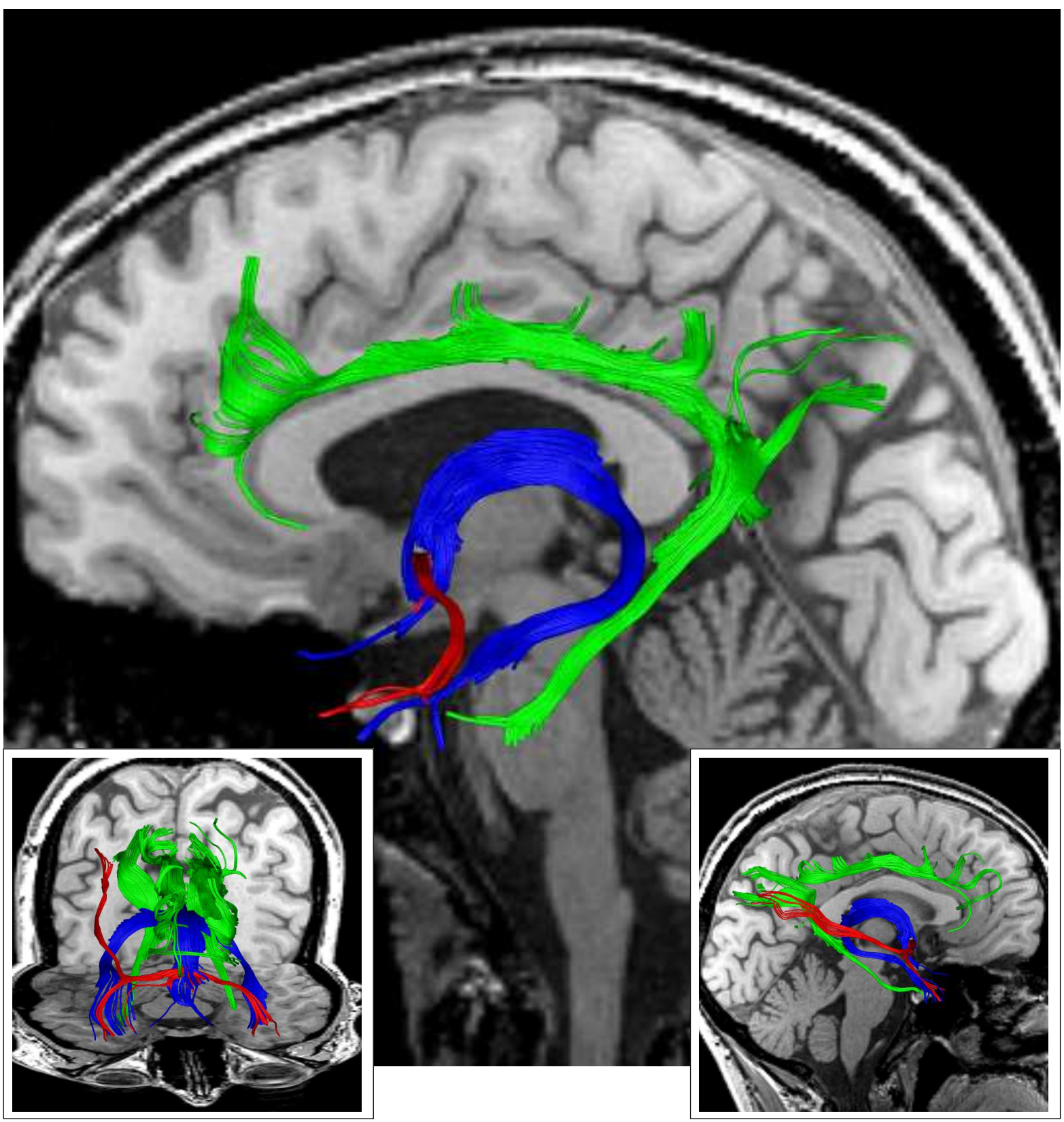

Abbildung 5.5: Limbisches System und Commissura anterior. Fornix (FX, Fasern des Cingulums ( $\mathrm{CG}$ ) und Commissura anterior ( $\mathrm{CA}$ ). 

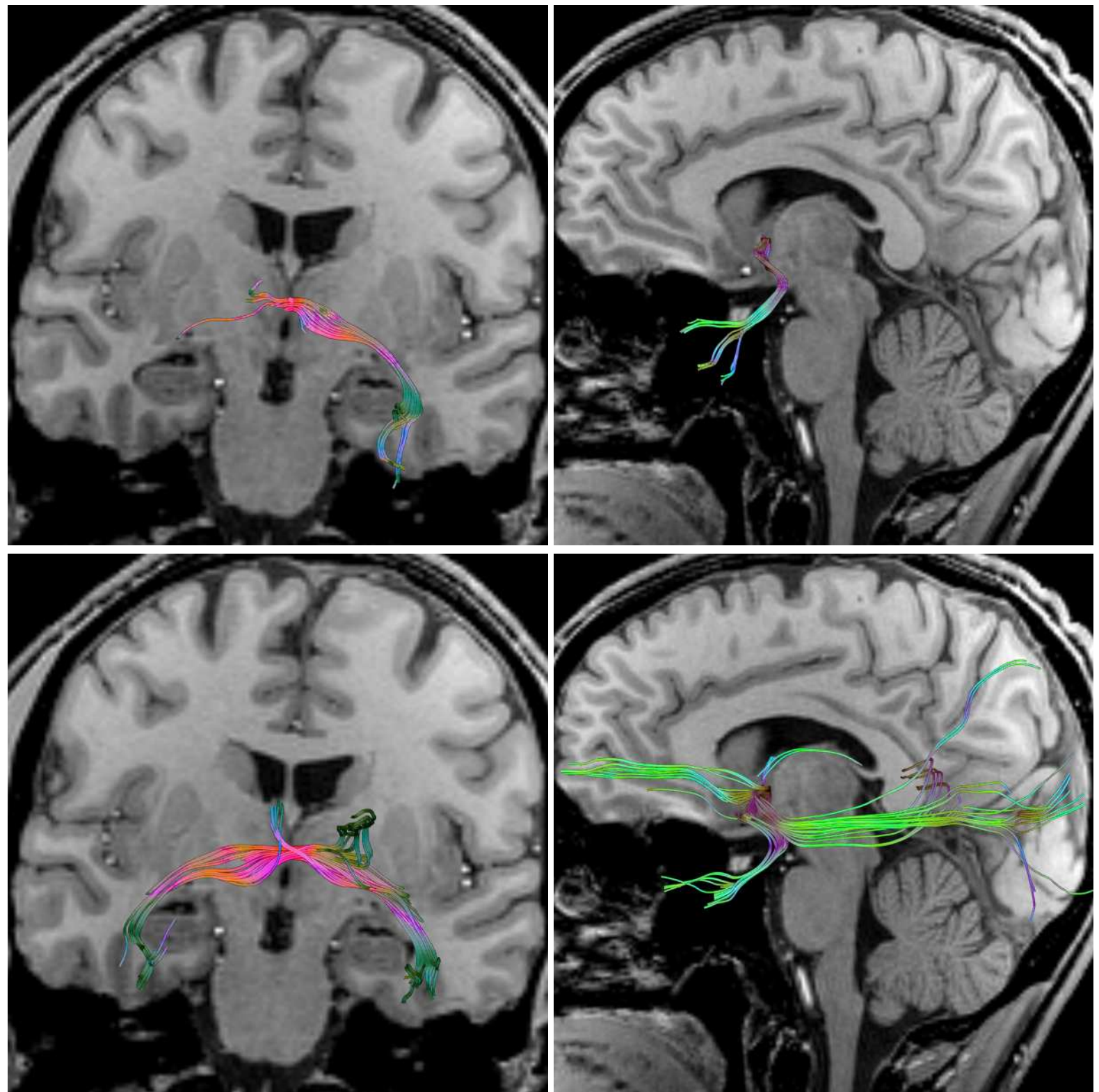

Abbildung 5.6: Commissura anterior. Vergleich des Tensorlines-Algorithmus (untere Reihe) mit FACT (oben). Es handelt sich um einen anderen Datensatz als in Abbildung 5.5. 
ne verlustreich, als daß er in seinem Verlauf sehr wahrscheinlich keine kurzreichweitigen Fasern beinhaltet. Das Gegenteil ist z. B. beim Fasciculus longitudinalis superior (FLS) der Fall. Dieser verbindet unter anderem die beiden nach Broca und Wernicke benannten Sprachzentren und umschließt die Insula-Region von oben in einem Bogen, der in der Capsula externa verläuft und schließlich in den Temporallappen mündet. Außerdem zieht ein Teil der Bahnen in den parietalen Cortex. Bemerkenswert ist eine Asymmetrie, die häufig bei der DTI-Traktographie auftritt: in der rechten Hemisphäre ist, wie am Beispiel in Abbildung 5.8 deutlich zu sehen, die Bogenstruktur oft nicht vollständig oder zumindest schlecht ausgeprägt. Dafür laufen dort vermehrt Fasern in den hinteren Bereich des Gehirns. Es erscheint nicht abwegig, diesen Umstand mit der bekannten Lateralisierung der Sprache, d. h. die einseitige Lokalisierung ihrer Funktionszentren in der zumeist linken Hemisphäre, in Zusammenhang zu bringen. Direkt neben dem Zweig des Fasciculus longitudinalis superior, der den Temporallappen erreicht, auch Fasciculus arcuatus genannt, wurde ein weiterer von oben nach unten laufender Axonverbund isoliert, der mit dem in einigen englischsprachigen Büchern erwähnten „perpendicular occipital fasciculus" (POF) identisch sein könnte, und der Nachbarschaftsbeziehungen zwischen kortikalen Regionen im Okzipitallappen herstellt. Wegen der unmittelbaren Nähe der beiden Faserbahnen sind Traktographieartefakte sehr wahrscheinlich nicht selten. Es stellt sich die Frage, ob die beobachtete Asymmetrie des FLS nicht wenigstens zum Teil auf diese Tatsache zurückzuführen ist. Außer den hier angeführten Assoziationsbahnen wird in einigen Arbeiten [102, 121] zur DTI-Traktographie auch ein in der Neuroanatomie als Fasciculus occipitofrontalis superior (FOFS) bezeichneter, wenig erforschter Trakt abgebildet. Er verläuft angeblich beidseitig medial von der Corona radiata unmittelbar unter dem Dach des Corpus callosums, was in dieser Arbeit aber nicht nachvollzogen werden konnte. Die bisherigen Darstellungen erscheinen wenig überzeugend und fußen unter Umständen auf überspringenden Fasern aus den Thalamusprojektionen. Darüberhinaus wird die Existenz des Fasciculus occipitofrontalis superior auch in anatomischen Kreisen angezweifelt (siehe [122]).

\subsubsection{Capsula interna (Crus anterius)}

Capsula interna ist der Name einer Region, die sich von unten her fächerförmig ausbreitende Fasern im Großhirn enthält. Diese verbinden subkortikale Zentren mit dem Cortex. Der hier interessierende vordere Teil wird lateral durch den Nucleus caudatus und das Putamen begrenzt, deren Ausdehnungen auch die Abmessung nach ventral und dorsal vorgeben. Er enthält frontal ausgerichtete Axonbahnen und ist deshalb technisch gesehen am besten mit einer koronal definierten Startregion zu erfassen. Das Ergebnis der Faserverfolgung ist in Abbildung 5.9 zu sehen. Man kann unschwer vier TrajektorienHäufungen ausmachen und diese mit Hilfe von multi-ROI-Verfahren voneinander trennen (Abbbildung 5.10). Dabei tritt topographisch eine gut definierte Schichtstruktur zutage. Gemäß der neuroanatomischen Literatur sind im Crus anterius der Capsula interna die Radiatio anterior thalami (RAT) und die Fibrae frontopontinae (FFP) angesiedelt, die auch erfolgreich rekonstruiert werden konnten. Daneben findet man mit der DTITraktographie auch Verbindungen ins Cerebellum und in den hinteren Bereich des Hirn- 


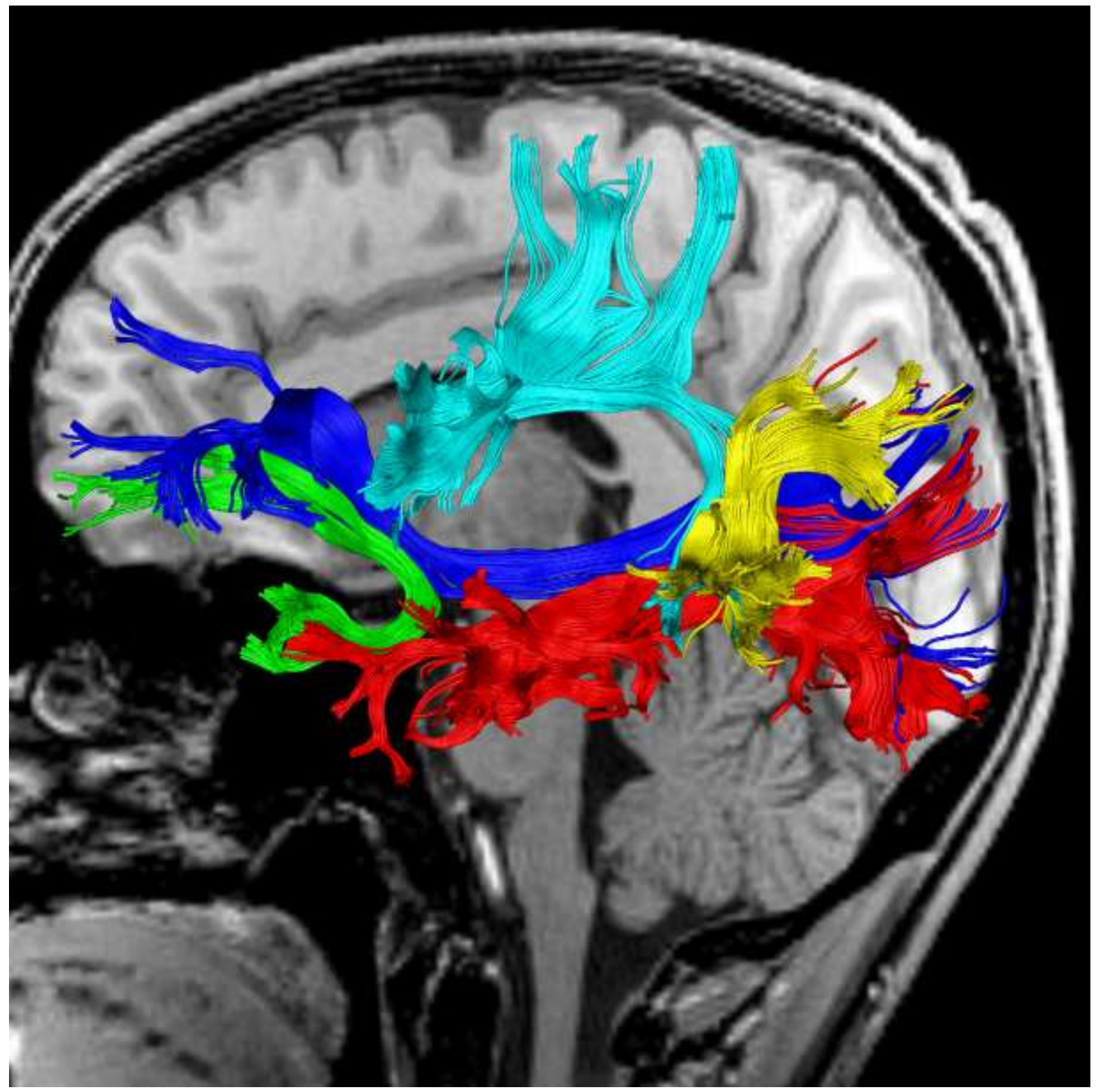

Abbildung 5.7: Assoziationsbahnen. Fasciculus longitudinalis inferior (FLI), Fasciculus uncinatus (FU), „perpendicular occipital fasciculus" ( POF ), Fasciculus occipitofrontalis (FOF) und Fasciculus longitudinalis superior (FLS). 

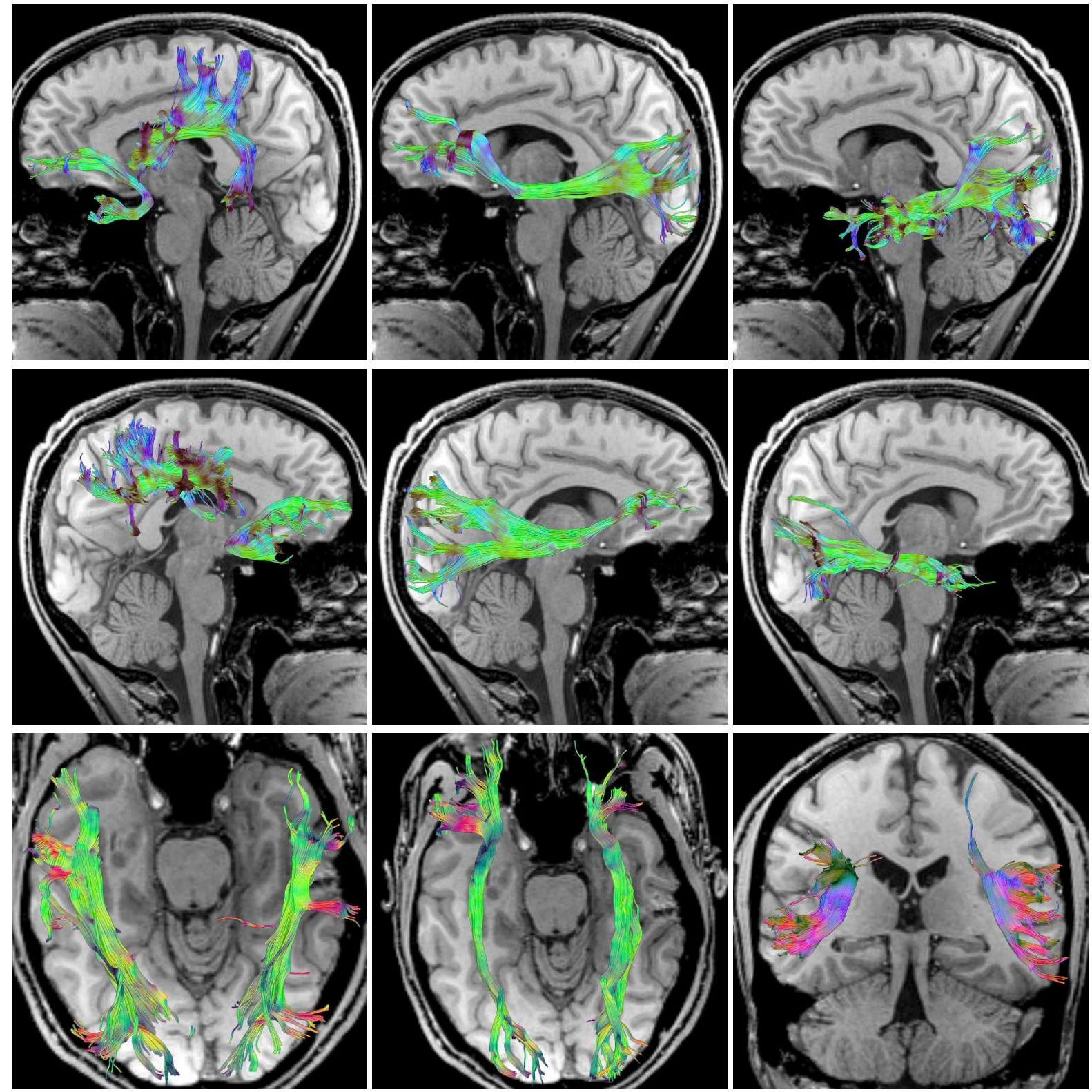

Abbildung 5.8: Symmetrie der Assoziationsbahnen. Die Beschreibung erfolgt von links nach rechts. Abkürzungen gemäß Abbildung 5.7. Die erste und zweite Reihe vergleichen sagittale Darstellungen von FLS\&FU, FOF und FLI jeweils von links (oben) und rechts (Mitte). Die unterste Zeile zeigt neben transversalen Ansichten von FLI und FOF auch den Verlauf des POF vor einer koronalen Schnittebene. 
stamms, die in dem entsprechenden Gebiet beide nahe des Nucleus ruber verlaufen. Sie sind möglicherweise mit den von Crosby [112, S.394-396] allerdings für das Genu der Capsula interna verzeichneten, korticorubralen und korticotegmentalen Axonbündeln in Verbindung zu bringen. Die DTI-Resultate legen deshalb eine Neubewertung oder zumindest vermehrten Aufklärungsbedarf über die wahre Topographie der Fasern in der Capsula interna nahe.

\subsubsection{Hirnstamm}

Legt man eine transversal orientierte Startregion in die sich unmittelbar an den Spinalkanal anschließende Medulla oblongata, lassen sich bereits einige der vielen den Hirnstamm bevölkernden Faserbündel identifizieren und im Anschluß mit der in diesem Bereich unabdingbaren multi-ROI-Methode gezielt isolieren (Abbildungen 5.11 und 5.12). Zielregionen im motorischen Cortex und im Kleinhirn (Cerebellum) separieren so beidseitig respektive den Tractus corticospinalis (TCS), auch Pyramidenbahn im engeren Sinne genannt [114, S.135], und das Pedunculus cerebellaris inferior (PCI). Eine eindeutige Trennung der im dorsalen Bereich verlaufenden Fasern des Fasciculus longitudinalis medialis/dorsalis (FL) von dem Lemniscus medialis (LM) ist erst ab dem Pedunculus cerebri (Crus cerebri und Tegmentum), einer oberen Grenze des Mittelhirns, gesichert, ab welcher der erstgenannte Trakt nach vorne in hypothalamische Regionen zieht (der mediale Anteil dient allerdings nur der Verknüpfung von Kerngebieten des Hirnstamms), während der LM eigentlich im weiter oben gelegenen Thalamus endet, aber über die sich anschließenden Bahnen letztendlich im Gyrus postcentralis auf den Cortex trifft. Latero-dorsal des Lemniscus medialis kann man eine weitere prägnante, durch das Diencephalon ziehende Faserstruktur ausmachen. Es handelt sich dabei wahrscheinlich um das in der anatomischen Literatur als Tractus spinothalamicus (TST) bekannte Axonbündel, das im Thalamus eine Verschaltung erfährt und danach ebenfalls zum Gyrus postcentralis weitergeleitet wird.

Die der Medulla oblongata angegliederte Pons ist Durchlauf-, Ursprungs- und Zielgebiet vieler Projektions- und Assoziationsbahnen. Prominent treten dort in einer sagittalen, farbkodierten MDD-Ansicht (siehe linke Spalte von Abbildung 5.1) in rot die großen Strukturen der ventralen und dorsalen Teile des Pedunculus cerebellaris medius (PCMV, PCM-D), einer Brücke zwischen den beiden Hälften des Cerebellums, hervor. Bei der Isolierung des Traktes kommt allerdings erschwerend hinzu, daß die Region von vielen Kernen grauer Substanz durchsetzt ist, die unter anderem auch kortikale Verbindungen haben. Außerdem passiert zwischen dem dorsalen und ventralen Teil des Pedunculus cerebellaris medius der Tractus corticospinalis. Zwar legt man die Startregion für die Fibrae frontopontinae besser, wie oben bereits geschehen, in den vorderen Teil der Capsula interna, man kann sie aber auch von der Pons aus verfolgen. Aufgrund der dort ausgeprägten komplizierten Gewebsorganisation sind auch die Crus cerebri (Hirnschenkel) geeigneter als Ausgangspunkt für die Darstellung der Fibrae occipito- und parietopontinae (FOPP). Als letzte hier vorgestellte, separat sinnvoll abbildbare Strukturen der weißen Substanz im Hirnstamm treten beidseitig die Pedunculi cerebellaris superior (PCS) aus dem Cerebellum in den Grenzbereich zwischen Mittelhirn und Diencephalon ein. 


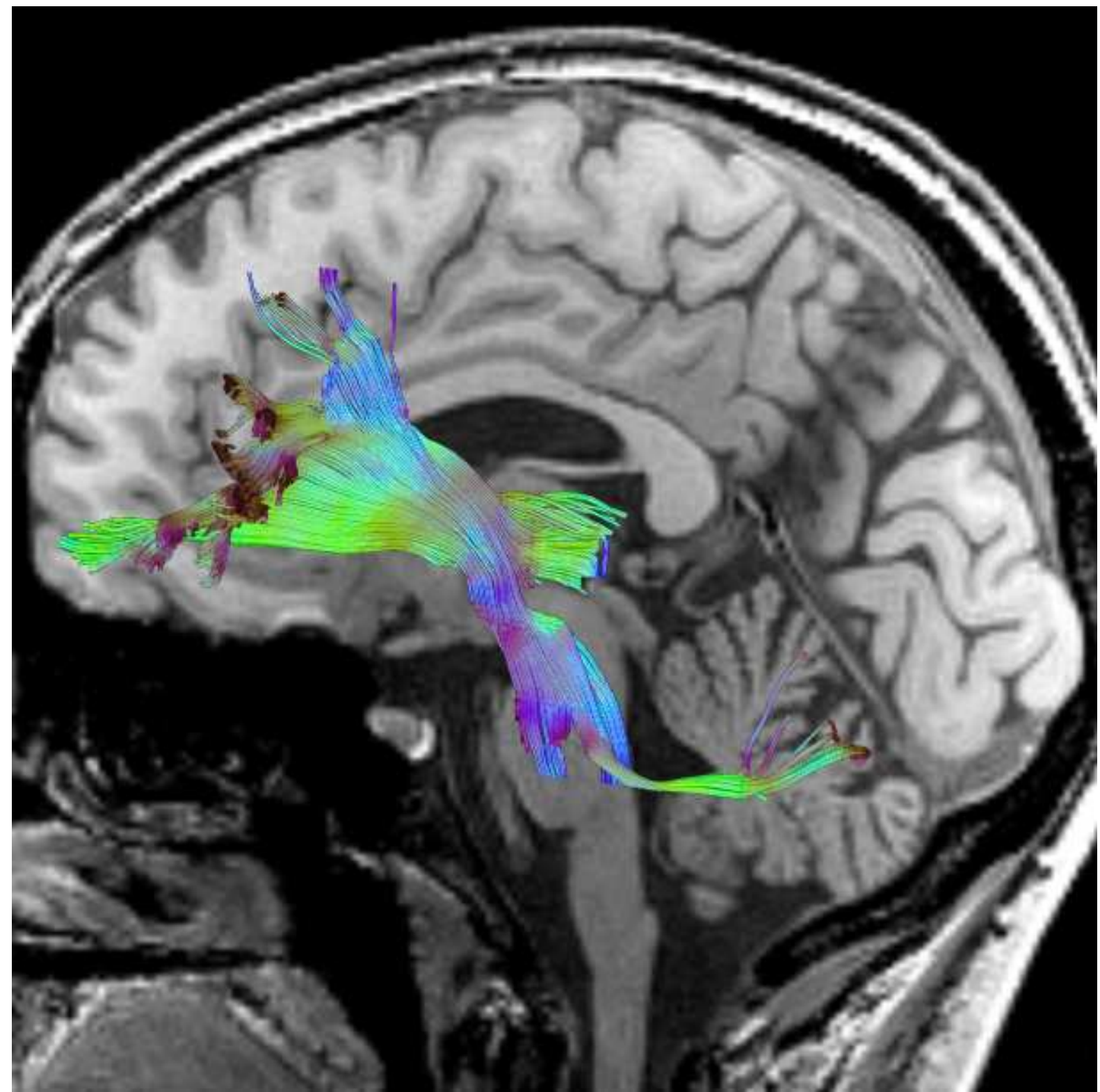

Abbildung 5.9: Crus anterius der Capsula interna. Weil das Meßprotokoll nur achtundreißig Schichten umfaßte, sind die Fasern nach unten hin abgeschnitten. 


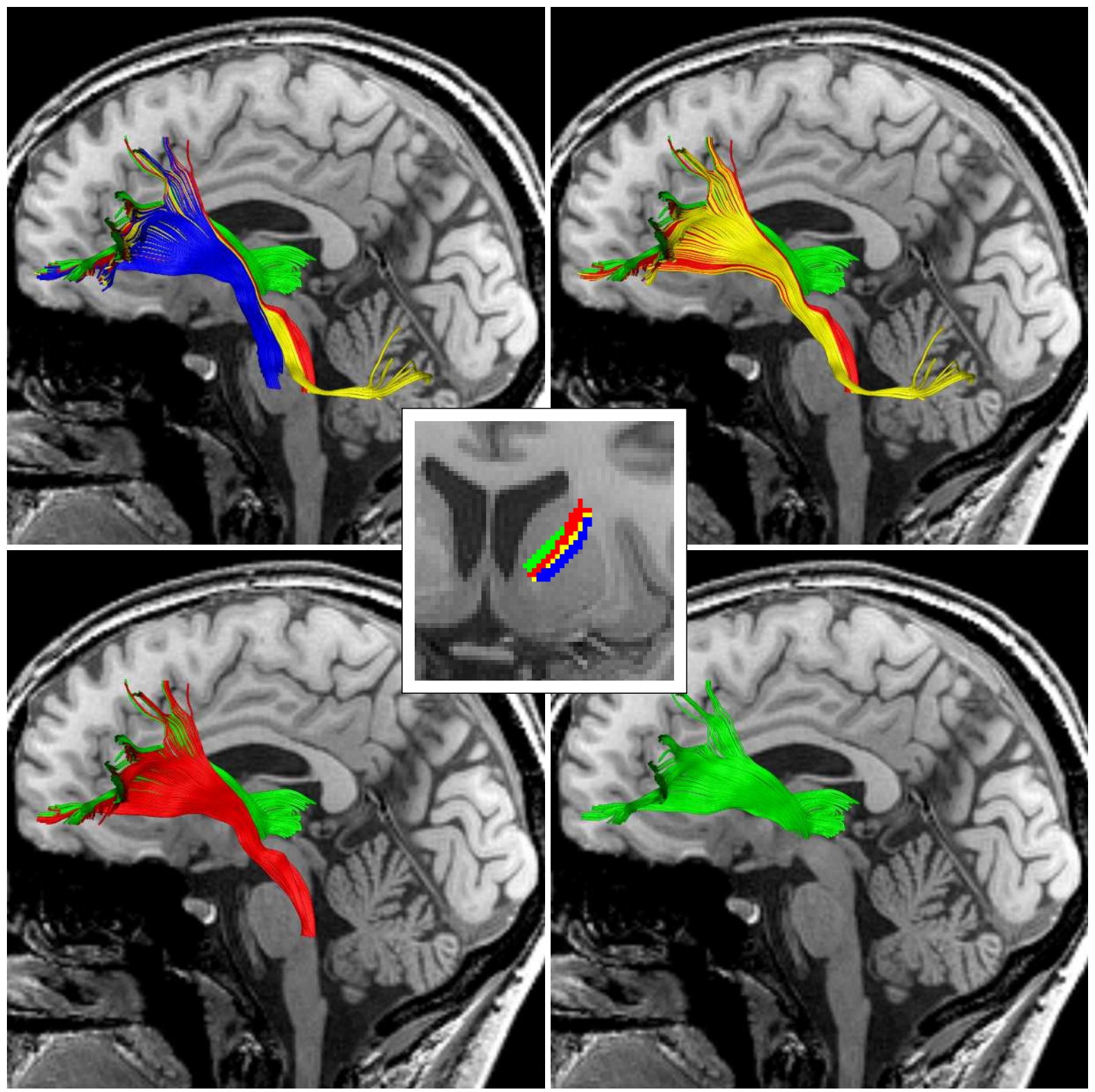

Abbildung 5.10: Topographie der Fasern durch das Crus anterius der Capsula interna. Die Schichtstruktur wird durch sukzessives Ablösen von isolierten Faserverbänden verdeutlicht. Fibrae frontopontinae (FFP), undokumentierte Verbindung in das Cerebellum (undokumentiert), undokumentierte Verbindung in den hinteren Hirnstammbereich (undokumentiert) und Radiatio anterior thalami (RAT). Kleines Bild: Trakt-zugehörige Pixel in einer koronalen Ansicht der vorderen Capsula interna. 
Die Rekonstruktionen der vorgestellten Faserbündel kommen der Beschreibung in der neuroanatomischen Literatur recht nahe. Allerdings treten auch gravierende Abweichungen vom als bekannt vorausgesetzten Verlauf auf. Am auffälligsten ist dabei die vollständige Abwesenheit von Faserkreuzungen des Tractus corticospinalis und des Lemniscus medialis bzw. der in ihm aufgehenden Axone im oberen Teil der Medulla oblongata, sowie des Pedunculus cerebellaris superior vor dem Eintreten in den Nucleus ruber. Die Begriffsbildung beim PCS ist allerdings verwirrend, da in manchen Atlanten der Eindruck entsteht, daß dessen Axone im Bereich des dorsalen Zweigs des Pedunculus cerebellaris medius verlaufen. Bemerkenswert ist auch, daß der Lemniscus lateralis, ein Teil des auditiven Apparates und eine der Hauptbahnen, nicht lokalisiert werden konnte, ebenso die von außen in den Hirnstamm eintretenden Hirnnerven des PNS. Anlaß zum Zweifel geben die Cortex-Anbindungen des rekonstruierten medialen Lemniscussystems, also der Lemniscus medialis und die Projektionen von seiner Eintrittsstelle in den Thalamus, die nicht in den somatosensorischen Gyrus postcentralis führen.

Zusammenfassend läßt sich sagen, daß DTI-Traktographie auf DW-SSSTEAM Daten im Hirnstamm möglich ist, aber in Anbetracht der zahlreichen dicht gepackten Axone im Moment ein Auflösungsproblem besteht. Selbst bei einer Überwindung dieser technischen Hürde muß eine zuverlässige Bildgebung dieser Region auf einem geeigneteren Diffusionsmodell aufbauen, das Partialvolumeneffekte im Zusammenhang mit Faserkreuzungen korrekt behandelt. Für Hirnpulsationen und Artefakte durch Probandenbewegungen ist der Hirnstamm zudem aufgrund seiner longitudinalen Durchsetzung mit dem vierten Ventrikel und seiner exponierten Lage besonders anfällig, was sich speziell auch auf DW-EPI Aufnahmen mit ihren sensiblen k-Raum-Trajektorien negativ auswirkt.

\subsubsection{Visuelles System}

Beginnend in den Sinneszellen der Retina verlaufen die Bahnen des visuellen Systems (Abbildungen 5.13 und 5.14) nach kleinskaliger Verschaltung durch Zwischenneurone zunächst im Nervus opticus (NO), der auch als zweiter Hirnnerv bezeichnet und damit dem periphären Nervensystem zugeordnet wird. Allerdings wächst er ontogenetisch aus dem Zwischenhirn heraus und hat auch entwicklungsgeschichtlich eher den Charakter einer zentralnervösen Faserstruktur. Das ist möglicherweise ein Grund, warum er hier mit der DTI-Traktographie erfaßt werden kann, während die übrigen Bahnen des PNS aus noch im Detail ungeklärter Ursache nahezu unsichtbar bleiben.

Im weiteren Verlauf des Nervus opticus, der im übrigen inklusive der ihn umgebenden Membrane nur 4-5 mm dick ist, trifft er im Chiasma opticum auf Axone des kontralateral gelegenen Sehnervs, wo sich die beiden berühren und die Anteile aus den medialen Netzhauthälften auf die jeweils andere Seite kreuzen. Den sich beidseitig kaudal anschließenden Teil der Sehbahn, in dem die Nerven wieder getrennt verlaufen, bezeichnet man als Tractus opticus (TO). Er endet im Corpus geniculatum laterale (CGL) des Thalamus, von wo aus in unmittelbarer Nachbarschaft die Fasern des letzten Teils der Sehbahn, als Radiatio optica (RO) bekannt, in den Okzipitallappen des Gehirns projizieren. Diese Sehstrahlung fächert von ihrem Ursprung im Thalamus aus sehr weit auf, so daß in der Nähe des CGL eine Vielzahl von Faserrichtungen zu beobachten ist. Einige Bahnen zielen 


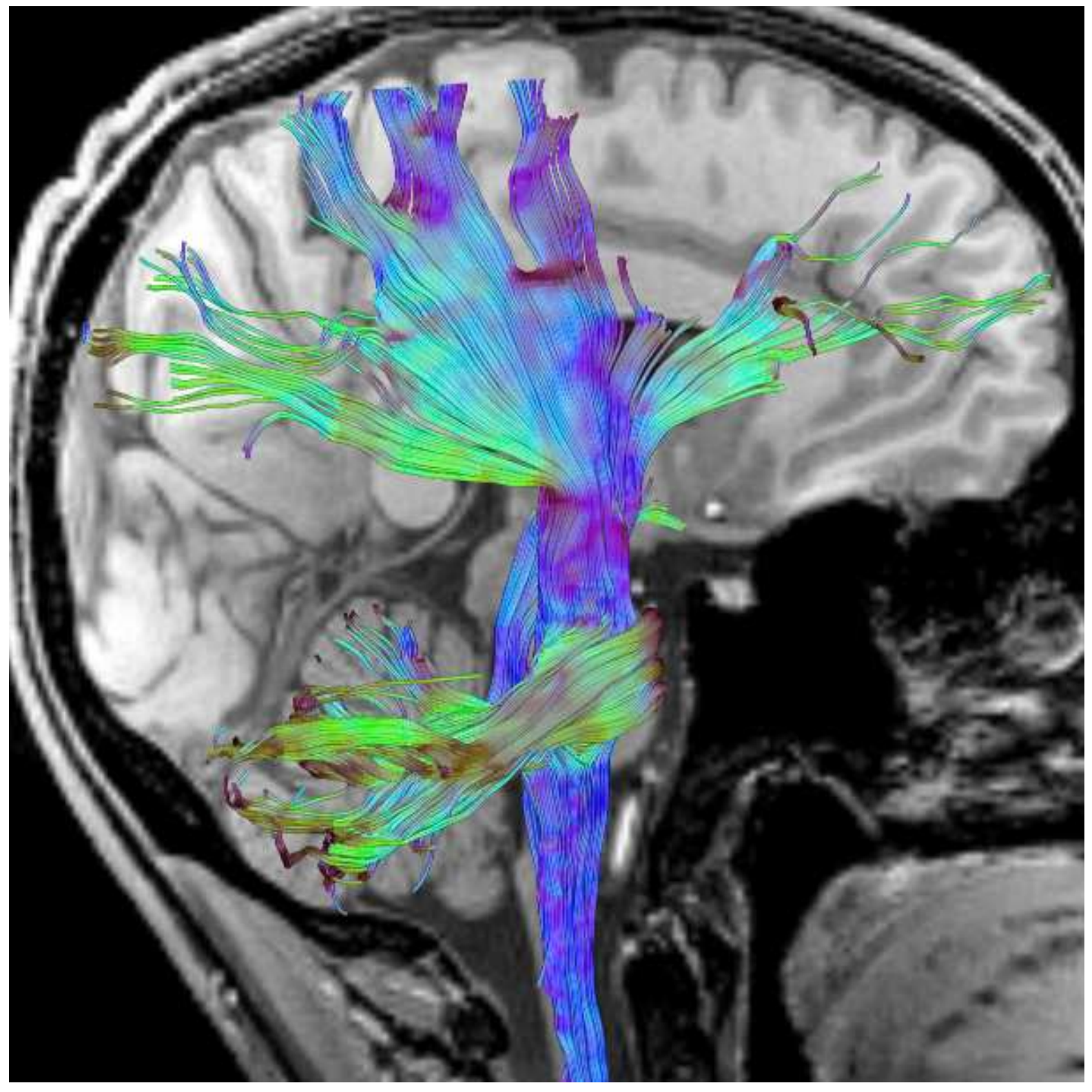

Abbildung 5.11: Hirnstamm. MDD-Ansicht aller FACT-Trajektorien. 

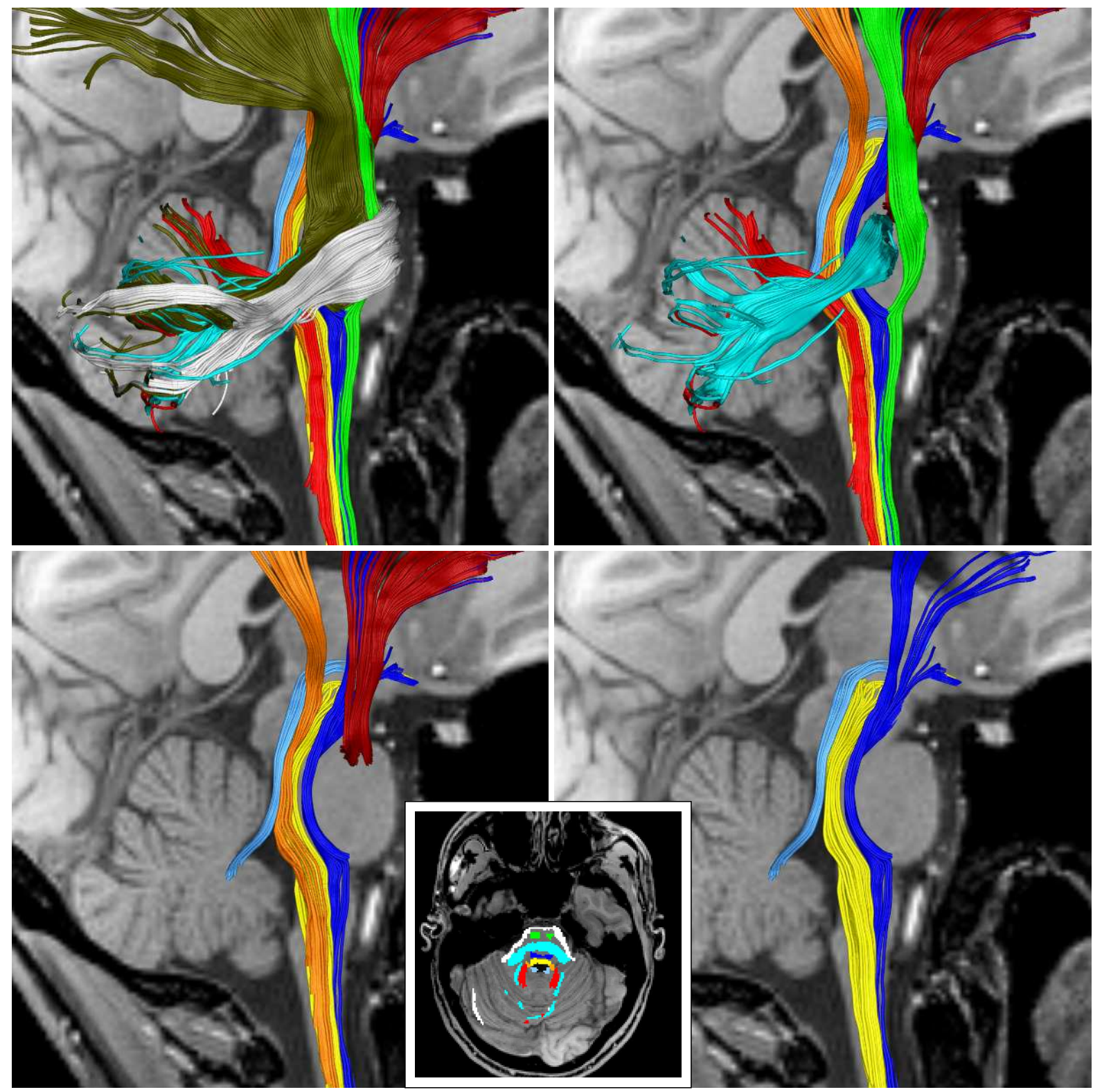

Abbildung 5.12: Hirnstamm Detailansicht. Pedunculus cerebellaris inferior ( PCI ), Tractus corticospinalis (TCS), Lemniscus medialis ( $\mathrm{LM}$ ), Pedunculus cerebellaris medius (dorsal) ( PCM-D ), Pedunculus cerebellaris medius (ventral) ( PCM-V $)$, Tractus spinothalamicus ( TST ), Pedunculus cerebellaris superior ( PCS ), Fasciculus longitudinalis (medialis und dorsalis) ( FL ), Fibrae occipito- und parietopontinae (FOPP ) und Fibrae frontopontinae (FFP). Kleines Bild: 2D Faseransicht über koronalem Schnitt durch Pons und Cerebellum. 
zwar relativ direkt in die Sehrinde, ein erheblicher Teil nimmt aber einen stark gekrümmten Umweg über den Temporallappen. Die Radiatio optica umkleidet „die Lateral- und Medialwände des Hinter- und Unterhorns des Seitenventrikels " [114].

Es gibt einige Berichte über Darstellungen des hinteren Teils der Sehbahn mit DTITraktographie [97, 123, 115, 124]. Das ist angesichts der Uneinheitlichkeit der Faserrichtungen am Corpus geniculatum laterale recht erstaunlich und vielfach lassen auch die Art der Abbildung (nur aktivierte Pixel, transversale Ansicht) oder starke Verzerrungen der Aufnahmen keine abschließende Beurteilung der Validität zu. In der vorliegenden Arbeit konnte die Radiatio optica nur in einem Probanden, und auch nur ein direkt verlaufendes Teilstück rekonstruiert werden (Abbildungen 5.13 und 5.14 links oben). Vielfach springen am CGL gestartete, kaudal verlaufende Bahnen entweder in das Corpus callosum oder den Fasciculus occipitofrontalis über und treffen im oberen Bereich des Okzipitallappens oder gar im Parietallappen auf den Cortex, nicht aber auf die Sehrinde. Daneben erschweren auch occipito- und parietopontine Bahnen aus dem Hirnstamm die Isolierung der hinteren Sehbahn. Seine Nähe zu den Ventrikeln macht den Bereich außerdem anfällig für Pulsationsartefakte.

Die DTI-Traktographie ist wegen der geringen Aufösung, der stumpfen Winkel und der Kreuzungen der Axone im Chiasma opticum nicht in der Lage, die dortige Fasertopographie anatomiegetreu aufzulösen. Auf Hindernisse anderer Natur stößt man bei der Verfolgung des Nervus opticus, der nämlich am Eingang zum Canalis opticus in die unmittelbare Nähe des Ursprungsrings der Augenmuskeln (Musculus rectus medialis, M. r. lateralis, M. r. inferior, M. r. superior) gerät und diesen durchstößt. Aufgrund ihrer ebenfalls faserigen Konsistenz werden die Muskeln gleichermaßen als anisotrope Strukturen erkannt, weil das DTI-Verfahren nicht spezifisch auf weiße Hirnsubstanz sondern auf die Gewebstextur sensibel reagiert. Wegen der ähnlichen Orientierung sind Übersprungsartefakte sehr häufig und auch deutlich in Abbildung $5.13 \mathrm{zu}$ beobachten. Dagegen zeigt das Beispiel einer anderen Messung in Abbildung 5.14, daß unter günstigen Voraussetzungen nicht nur der Verlauf des Sehnervs allem Anschein nach korrekt dargestellt, sondern auch Teile der umgebenden Muskulatur visualisiert werden können.

Die Region um den Nervus opticus entzieht sich einer Abbildung mit der üblichen EPI-DTI-Aufnahmetechnik wegen ihrer ausgeprägten Verzerrungen. Für alle Aufnahmemethoden problematisch ist die Tatsache, daß der Nerv bei Augenbewegungen leicht verschoben wird. In auf „Fast Spin-Echo “-Sequenzen (FSE) basierenden Arbeiten [125, 126] gelang unter Verwendung angepaßter Spulentechnik, Schichtorientierung und -auflösung bisher zwar die Erstellung von ADC- und FA-Karten, nicht aber die Rekonstruktion des Verlaufs mittels Traktographie. Um so erstaunlicher ist die Tatsache, daß dies mit DWSSSTEAM (FF+R2-e/Lorentz), Standard-Hardware und ohne besondere weitere Vorkehrungen erstmalig möglich geworden ist. Hinsichtlich der inter- und intra-individuellen Reproduzierbarkeit der Ergebnisse liegen allerdings noch keine Erkenntnisse vor. 


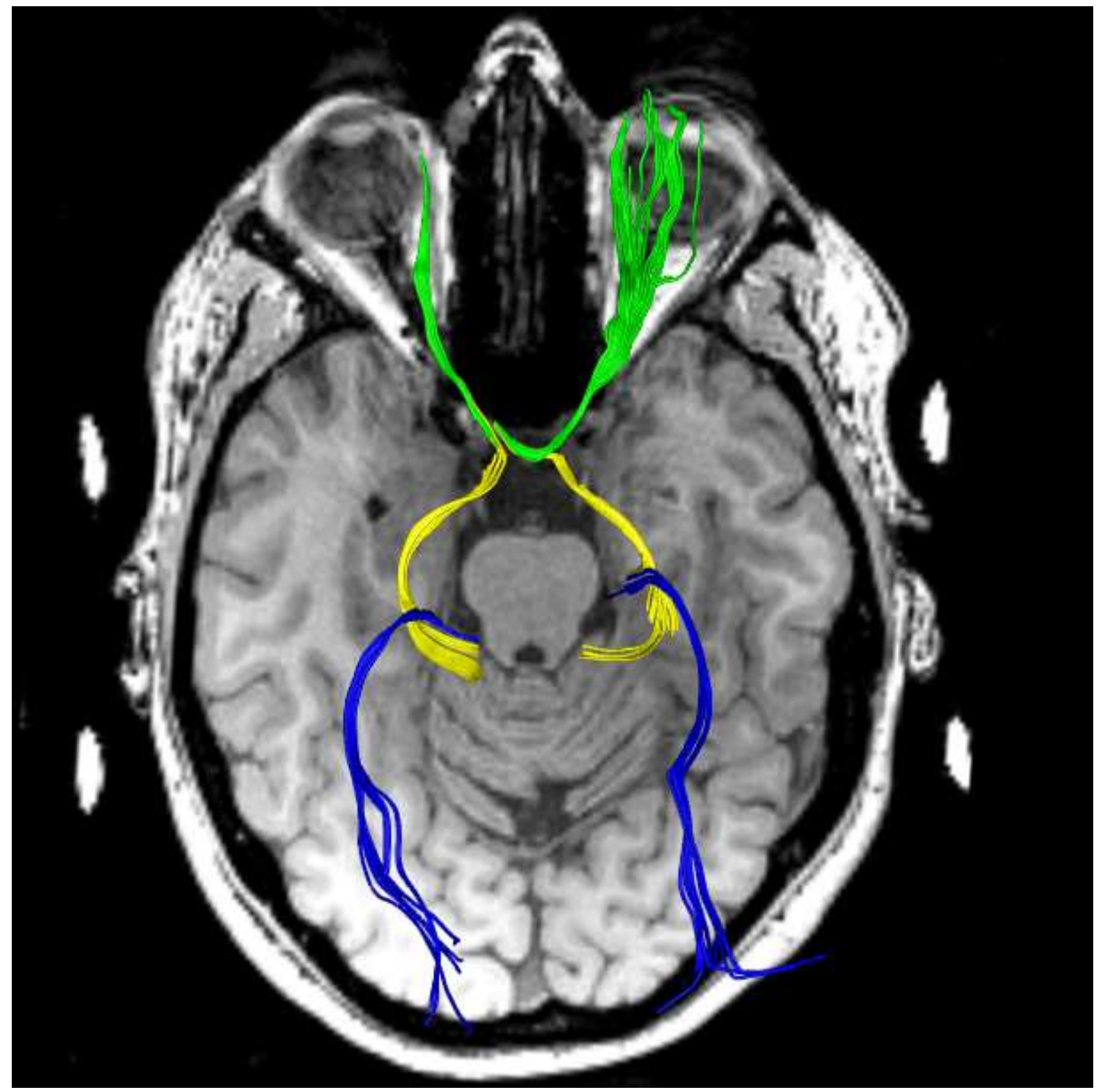

Abbildung 5.13: Visuelles System. Nervus opticus (NO), Tractus opticus ( TO ) und Radiatio optica (RO). 

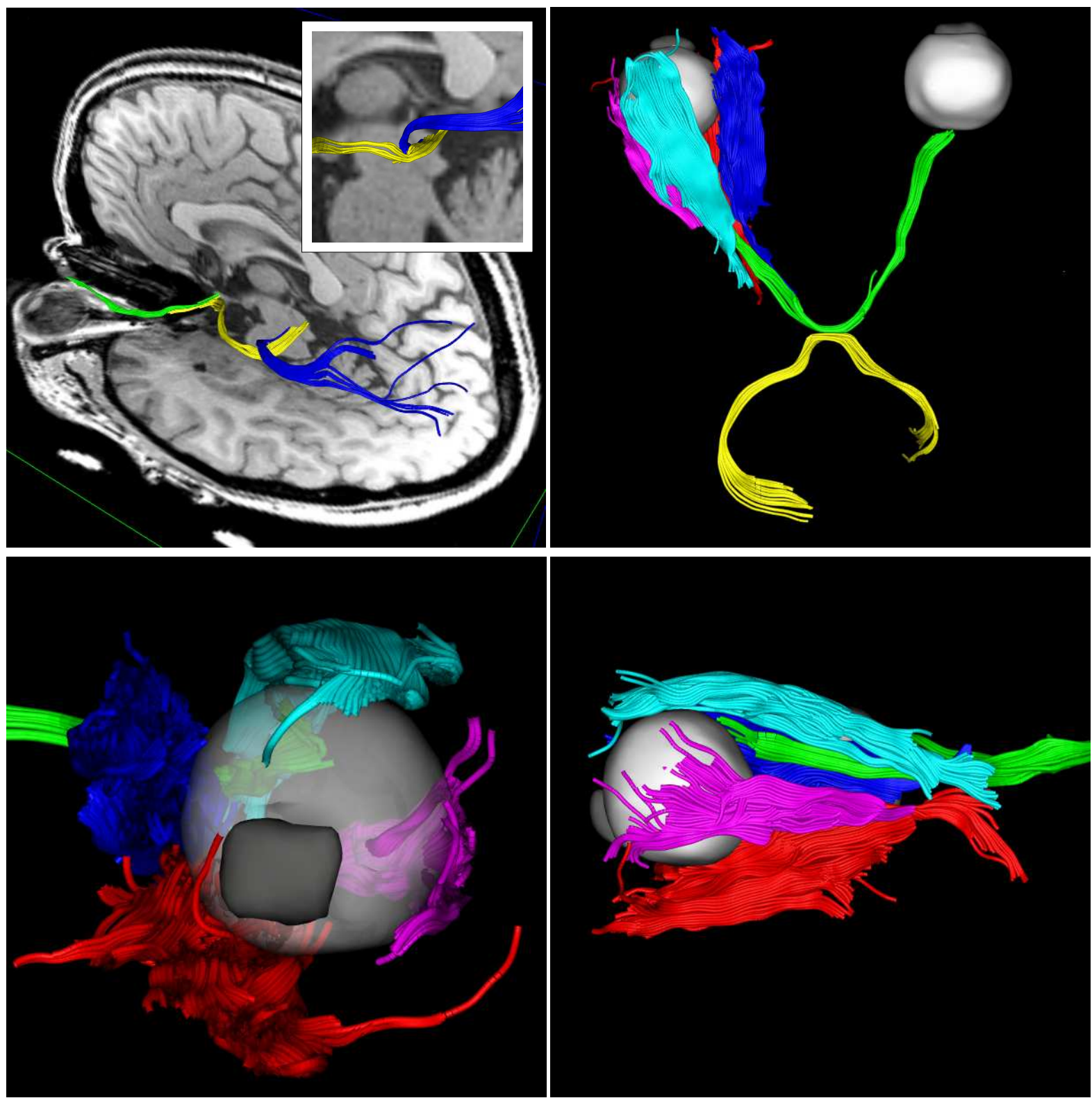

Abbildung 5.14: Visuelles System und Augenmuskulatur. Nervus opticus ( NO), Tractus opticus ( TO ), Radiatio optica/Musculus rectus medialis ( RO/MRM), Musculus rectus lateralis (MRL), Musculus rectus superior ( MRSUP ) und Musculus rectus inferior (MRINF ). Zur besseren Illustration sind Darstellungen von den Augäpfeln (weiß) und den Linsenregionen (grau) beigefügt. Die linke obere Aufnahme entspricht einer anderen Ansicht der Rekonstruktion aus Abbildung 5.13 und entstammt aus einer Akquistion mit der DW-SSSTEAM Referenzimplementation. Die restlichen Bilder beruhen auf Daten, die mit der FF+R2-e Variante gewonnen wurden. 


\section{KAPITEL 6}

\section{Abschließende Anmerkungen}

Die in dieser Arbeit vorgenommene Erweiterung der DW-SSSTEAM-Sequenz um die Anwendbarkeit paralleler Bildgebung hat sich als fruchtbarer Weg erwiesen, die Aufnahmedauer im Vergleich zur Partial Fourier Referenzsequenz ohne Qualitätseinbußen zu verkürzen. Für den mit den bestehenden Mitteln erreichbaren Reduktionsfaktor $R=2$ beträgt die Meßzeit-Ersparnis ca. 17\%, im Vergleich zur unbeschleunigten Full-FourierAkquisition sogar 45\%. Infolgedessen sinkt zum einen die Anfälligkeit gegenüber Bewegungsartefakten, zum anderen steigt damit die Akzeptanz im klinischen Umfeld. Als positiver Nebeneffekt des zeitlich reduzierten Ausleseabschnittes und der begleitenden Erhöhung der Flipwinkel vermindert sich außerdem die Gefahr von störenden Echos aufgrund von Anregung und Refokussierung $T_{1}$-relaxierender Magnetisierung.

Zur weiteren Reduktion der Meßzeit auf 302 ms pro Bild wurde die Kombination von paralleler Bildgebung und Partial Fourier Technik realisiert. Obwohl sie prinzipiell einsetzbar ist, gibt es bei dieser Variante noch weiteren Forschungsbedarf hinsichtlich der Rekonstruktion, die zwei verschiedenen k-Raum-Reduktionsmechanismen gerecht werden muß.

In theoretischen und experimentellen Untersuchungen stellte sich heraus, daß es bei Fortschritten in der Verbesserung der PPI-Rekonstruktionsgüte im Hinblick auf die Rauschverstärkung durch Linienauslassung, die zur Zeit über dem theoretischen Minimum liegt, noch Spielraum für eine Verbesserung des SNR gibt. Bei der gewählten Auflösung und Matrixgröße muß man für den sinnvollen Einsatz des nächsthöheren Reduktionsfaktors $R=3$, der neben weiterer Meßzeitverkürzung auch einen zusätzlichen Qualitätsgewinn verspricht, sehr wahrscheinlich auf Spezialspulen zurückgreifen, die für die Zwecke paralleler Bildgebung optimiert worden sind. Obwohl in den PPI-beschleunigten Bildern keine Verschmierungen auftauchen, sollte vor einer weitergehenden Parallelisierung überprüft werden, inwiefern die GRAPPA-Rekonstruktion die durch die Flipwinkelfunktion aufgeprägte Punkt-Transfer Funktion erhält. Einstweilen bietet nach den Ergebnissen aus Kapitel 4 die Verringerung der Aufnahmebandbreite eine Möglichkeit, das SNR der Bilder im Tausch gegen Meßzeit weiter zu steigern. 
Trotz der erzielten Verbesserungen erscheint die in-vivo Verwendung von fortschrittlicheren Verfahren zur Aufklärung der Gewebsarchitektur wie Q-Ball-Imaging [29] in Kombination mit der DW-SSSTEAM-Sequenz unter den gegebenen Voraussetzungen nicht nur aufgrund ihres zeitlichen Umfangs unrealistisch. Zum einen erfordern diese Techniken hochwinkelaufgelöste Daten, wodurch sich wegen der Richtungsabhängigkeit der erzielbaren Magnetfeldgradientenamplitude eines NMR-Gerätes die Diffusionspräparationszeit verlängern würde, mit wegen $T_{2}$-Zerfalls nachteiligen Folgen für die Signalstärke. Zum anderen und mit im Endeffekt gleichen Konsequenzen muß dabei die Diffusionswichtung verstärkt werden. Dafür reicht das verfügbare SNR bei weitem nicht aus. Für eine unverfälschte Messung sollte nämlich das theoretische, diffusionsgewichtete Signal, $S_{0} e^{-b D}$, zumindest über dem Rauschuntergrund liegen. Nimmt man für eine limitierende Abschätzung den größten vorkommenden Diffusionskoeffizienten entlang der Faserrichtung in der weißen Substanz des menschlichen Gehirns von ca. $D=2 \cdot 10^{-3} \mathrm{~mm}^{2} / \mathrm{s}$ und setzt einen b-Wert von $1000 \mathrm{~s} / \mathrm{mm}^{2}$ voraus, so muß nach Formel (3.13b) in Magnitudenbildern

$$
\frac{S_{0}}{\sigma} \geq \sqrt{\frac{\pi}{2}} e^{b D}
$$

gelten [50]. Das bedeutet bei den gewählten Werten $\mathrm{SNR} \geq 9,3$. Ruft man sich mit der Vorgabe dieses Maßstabs den in Kapitel 4 ermittelten Wert von SNR $\approx 7$ ins Gedächtnis, reicht die Bildqualität der DW-SSSTEAM-Sequenz noch nicht einmal für die Diffusionstensorbildgebung völlig aus. Um eine im obigen Sinne korrekte Schätzung der Diffusionskoeffizienten zu erhalten müßte der maximale b-Wert auf ca. $860 \mathrm{~s} / \mathrm{mm}^{2}$ sinken. Abmildernd muß hier hinzugefügt werden, daß gemäß dieser Argumentation in einem Großteil des Zielgewebes mit typischerweise $D \approx 1 \cdot 10^{-3} \mathrm{~mm}^{2} / \mathrm{s}$ schon ein SNR-Niveau von 3,4 für eine korrekte Berechnung ausreicht. Zwar stellt Gleichung (6.1) eine natürliche untere Schranke für das in quantitativen Untersuchungen notwendige Signal-zu-RauschVerhältnis dar, weil aber die Rice-Verteilung nur im Limes großer Signalstärken in eine Gauß-Kurve übergeht, werden insbesondere die diffusionsgewichteten Intensitäten in der Nähe des Rauschuntergrundes immer noch nach oben hin verfälscht.

Wegen der in den mit POCS rekonstruierten Partial Fourier Bildern beobachteten Anomalie bezüglich der Statistik der Intensitätswerte (siehe Abschnitt 4.6) ist es wohl allgemein und gerade bei mit k-Raum-reduzierten Verfahren gemessenen Magnitudenbildern angeraten, neben dem wahren SNR auch den Rauschuntergrund zur Qualitätsmessung von Diffusionssequenzen heranzuziehen. Die Bestimmung des Signal-zu-RauschVerhältnisses sollte, wie in Abschnitt 4.6 durchgeführt, über eine Ensemble-Messung in hinreichend signalintensiven Objekt-Voxeln erfolgen.

Die vorangegangene Argumentation macht deutlich, daß auch eine Auflösungserhöhung angesichts der aktuell erreichbaren Bildqualität nicht realistisch ist, und daß sogar über eine moderate Voxelvergrößerung nachgedacht werden muß, um zuverlässig quantitative Studien betreiben zu können. Die Wahl von isotropen Bildelementen mit einer Kantenlänge von 2,5 mm verdoppelt z. B. nahezu das zu erwartende Signal. Außerdem ist zu klären, warum die mit DW-SSSTEAM erzielten FA-Werte im Vergleich zu den Ergebnissen aus SNR-stärkeren EPI-Akquisitionen geringer ausfallen. 
Ein weitgehend ungelöstes Problem von Diffusionstensormessungen stellen Artefakte durch Probandenbewegungen dar, denen wohl zufriedenstellend nur durch zusätzliche Lokalisierungsmessungen (Navigatoren) und Echtzeit-Korrekturen an den Gradienten mit großem technischen Aufwand beizukommen wäre. Aufgrund der massiv unterschiedlichen Bildkontraste und der durch die Diffusionsgradienten ausgezeichneten Richtung bietet eine Koregistrierung der Daten auf die ungewichteten Messungen auch nur einen eingeschränkten Nutzen. In EPI-Aufnahmen ist außerdem wegen Wirbelströmen eine diffusionsrichtungsabhängige Verzerrung zu verzeichnen. Die konzeptionell beste Art, Bewegungsartefakte zu minimieren, besteht in einer schnellen Messung. Zur Vermeidung von Bildbeeinträchtigungen durch Hirnpulsationen und den dadurch in ventrikelnahen Bereichen sowie im Hirnstamm entstehenden Fehlern bei der Berechnung der Hauptdiffusionsrichtung wäre es vorteilhaft, die Messung mit der Herzaktivität des Probanden zu synchronisieren [127]. Auch aus diesem Grund ist eine Realisierung von DW-SSSTEAM mit einem Reduktionsfaktor von $R=3$ und einer Aufnahmezeit von $286 \mathrm{~ms}$ pro Bild bei $180 \mathrm{~Hz} /$ Pixel Aufnahmebandbreite sehr erstrebenswert, weil man dann mit zwei Bildern pro Herzschlag den verfügbaren Rahmen von etwa 600 ms (siehe Abschnitt 4.3.2) optimal ausnutzen könnte.

In den Untersuchungen zur Eignung von DW-SSSTEAM-Daten für die Faserverfolgung stellte sich heraus, daß die Ergebnisse, soweit vergleichbar, eine gute Übereinstimmung mit den aus EPI-Messungen gewonnenen Resultaten zeigen, sofern es sich um nicht verzerrungsanfällige Regionen handelt. Neben den bereits publizierten Assoziationsbahnen konnte darüberhinaus der „perpendicular occipital fasciculus“ isoliert werden. Bisher einmalig ist die Rekonstruktion des Nervus opticus und der ihn umgebenden Muskeln. Ebenso hat die Qualität und Detailtreue der mit DW-SSSTEAM abbildbaren Fasern im Hirnstamm als ebenso sensibler Region in der DTI-Literatur zur Zeit kein Äquivalent. Einige Strukturen und Einzelheiten entzogen sich allerdings der Rekonstruktion, und der Verlauf mehrerer Trajektorien ist in Teilen zweifelhaft bzw. weicht von der Literaturbeschreibung ab.

Trotz der vielversprechenden Resultate bei der Segmentierung der Capsula interna muß angemerkt werden, daß die Exaktheit der Darstellung von Axonbündeln nahe den Ventrikeln von einer EKG-Synchronisation der Sequenz profitieren dürfte. Die bisherige DTI-Analyse hat neben der Bestätigung der schichtartigen Struktur der parallel gepackten Frontalprojektionen durch das Crus anterius außerdem ergeben, daß möglicherweise nicht nur wie bisher angenommen die Fibrae frontopontinae und die vordere thalamische Radiation diesen Bereich der weißen Substanz ausmachen, sondern ebenso weiter kaudal geglaubte Bahnen.

Neben den Artefakten auf der Meßebene schlägt sich auch das Versagen des Diffusionstensormodells in Regionen mit Faserkreuzungen deutlich in den Ergebnissen nieder. Das wurde am Beispiel der fehlenden Lateralprojektionen in den mit dem Standard-FACTVerfahren nachvollzogenen Bahnen des Corpus callosums diskutiert. Die Frage nach dem sinnvollen Einsatz von stärker regularisierenden Algorithmen zur Behebung solcher Probleme sollte in dieser Arbeit nur angesprochen, aber nicht erschöpfend behandelt werden. Das Tensorlines-Verfahren liefert bei der Commissura anterior überzeugende Ergebnisse. Auch einige der gesuchten Verbindungen im Balken können anscheinend nachvollzogen 
werden. Der Algorithmus vermeidet dafür aber andere potentiell richtige Trajektorien. Die Ergebnisse sind außerdem stark von der Größe des Parameters $w$ abhängig. Die durch dessen Einstellung getroffenen Annahmen über die Steifigkeit einer Bahn lassen sich nicht verallgemeinern, denn unter denselben Bedingungen wie bei der Commissura anterior schlägt z. B. die Rekonstruktion des Fornix größtenteils fehl. Aufgrund der komplexen Verhältnisse im menschlichen Gehirn erscheint es daher zweifelhaft, ob eine tragfähige Lösung der mangelnden Darstellbarkeit von Faserkreuzungen in DTI-Daten auf algorithmischer Ebene gefunden werden kann.

Bis heute ist es aus der Sicht des Autors noch nicht gelungen, die Resultate von DTIFaserverfolgungsalgorithmen sinnvoll zu quantifizieren. Vor der Klärung dieser konzeptionellen Frage muß zuallererst die Reproduzierbarkeit der Technik überprüft werden. Ist diese gegeben, können die Trajektorien von Axonbündeln zur Definition von ROIs dienen, mit denen dann über die skalaren Karten der Diffusionstensorbildgebung oder anderer koregistrierter Bilder eine aussagekräftige quantitative Auswertung möglich ist, z. B. haben sich Anisotropiemaße als sensible Indikatoren bei neurodegenerativen Erkrankungen herausgestellt. Davon abgesehen lassen sich auch aus dem qualitativen Faserverlauf neue Einsichten in alte Fragestellungen gewinnen. So bemüht man sich schon seit längerem um eine Segmentierung des Corpus callosums anhand der funktionell abgrenzbaren Regionen, die dessen Fasern verbinden. Erste Untersuchungen mittels DTI-Traktographie deuten darauf hin, daß das bisher verwendete Parzellierungsschema, das zudem von Primatenstudien auf den Menschen extrapoliert wurde, der Korrektur bedarf. Außerdem ist die genaue Lage selektiver axonaler Verknüpfungen von großem Wert für den Neurochirurgen, der möglicherweise in Zukunft anhand von Traktographie-Resultaten bei Tumoroperationen sein Vorgehen präoperativ plant und postoperativ kontrolliert, oder sogar interventionell während des Eingriffs Faserbündel verfolgen kann. Die DW-SSSTEAM-Sequenz ist prinzipiell durch ihre anatomiegetreuen Abbildungseigenschaften für geometrisch sensible Anwendungen prädestiniert. Für den Einsatz im Operationssaal sollte aber nach weiteren Möglichkeiten zur Verkürzung der Meßdauer gesucht werden. 
Appendix 



\section{ANHANG A}

\section{Sequenzeinstellungen}

\section{Parameter}

\begin{tabular}{|c|c|c|}
\hline Parameter & \multicolumn{2}{|c|}{ Wert } \\
\hline \multicolumn{3}{|r|}{ Bildgebung } \\
\hline Auflösung & \multicolumn{2}{|c|}{$2 \times 2 \times 2 \mathrm{~mm}^{3}$} \\
\hline $\mathrm{TE}_{\mathrm{SE}}$ & \multicolumn{2}{|c|}{$50,10 \mathrm{~ms}$} \\
\hline $\mathrm{TE}_{\mathrm{STE}}$ & \multicolumn{2}{|c|}{$8,70 \mathrm{~ms}$} \\
\hline $\mathrm{TR}_{2}$ & \multicolumn{2}{|c|}{$7,81 \mathrm{~ms}$} \\
\hline $\mathrm{TR}_{1}$ & \multicolumn{2}{|c|}{$700 \mathrm{~ms}(\mathrm{FF})$} \\
\hline & \multicolumn{2}{|c|}{$466 \mathrm{~ms}(\mathrm{PF} 5 / 8)$} \\
\hline & \multicolumn{2}{|c|}{$380 \mathrm{~ms}(\mathrm{FF}+\mathrm{R} 2-\mathrm{e})$} \\
\hline & \multicolumn{2}{|c|}{$302 \mathrm{~ms}$ (PF 6/8+R2-e) } \\
\hline$B W$ & \multicolumn{2}{|c|}{$180 \mathrm{~Hz} /$ Pixel } \\
\hline$N_{\mathrm{av}}$ & \multicolumn{2}{|c|}{2} \\
\hline PSF & \multicolumn{2}{|c|}{$\begin{array}{c}\text { Lorentz (variable Flipwinkel, } T_{1}=700 \mathrm{~ms} \text { ) } \\
\text { PPI (extern) }\end{array}$} \\
\hline$R$ & 2 & \\
\hline \multirow[t]{2}{*}{$N_{\text {ref }}$} & 25 & \\
\hline & & Diffusion \\
\hline$\delta_{\mathrm{D}}$ & \multicolumn{2}{|c|}{$17,42 \mathrm{~ms}$} \\
\hline$\Delta_{\mathrm{D}}$ & \multicolumn{2}{|c|}{$33,11 \mathrm{~ms}$} \\
\hline$b$ & \multicolumn{2}{|c|}{$0 \mathrm{~s} / \mathrm{mm}^{2}$ und $1000 \mathrm{~s} / \mathrm{mm}^{2}$} \\
\hline
\end{tabular}

Tabelle A.1: Standard Sequenzparameter. Experimentell verwendete Konfigurationen der DW-SSSTEAM-Sequenz. Die Erwähnung von FF und PF 6/8+R2-e dient nur dem Vergleich. 


\section{Diffusionsrichtungen}

\begin{tabular}{lccc}
\hline Gradientenindex & $x$ & $y$ & $z$ \\
\hline 1 & $+0,618034$ & $+0,000000$ & $-1,000000$ \\
2 & $-0,618034$ & $-0,000000$ & $+1,000000$ \\
3 & $+0,618034$ & $+0,000000$ & $+1,000000$ \\
4 & $-0,618034$ & $-0,000000$ & $-1,000000$ \\
5 & $+0,000000$ & $+1,000000$ & $-0,618034$ \\
6 & $-0,000000$ & $-1,000000$ & $+0,618034$ \\
7 & $+0,000000$ & $+1,000000$ & $+0,618034$ \\
8 & $-0,000000$ & $-1,000000$ & $-0,618034$ \\
9 & $+1,000000$ & $-0,618034$ & $+0,000000$ \\
10 & $-1,000000$ & $+0,618034$ & $-0,000000$ \\
11 & $+1,000000$ & $+0,618034$ & $+0,000000$ \\
12 & $-1,000000$ & $-0,618034$ & $-0,000000$ \\
13 & $+0,000000$ & $+0,618034$ & $-1,000000$ \\
14 & $-0,000000$ & $-0,618034$ & $+1,000000$ \\
15 & $+0,000000$ & $+0,618034$ & $+1,000000$ \\
16 & $-0,000000$ & $-0,618034$ & $-1,000000$ \\
17 & $+0,618034$ & $+1,000000$ & $+0,000000$ \\
18 & $-0,618034$ & $-1,000000$ & $-0,000000$ \\
19 & $+0,618034$ & $-1,000000$ & $+0,000000$ \\
20 & $-0,618034$ & $+1,000000$ & $-0,000000$ \\
21 & $+1,000000$ & $+0,000000$ & $+0,618034$ \\
22 & $-1,000000$ & $-0,000000$ & $-0,618034$ \\
23 & $+1,000000$ & $+0,000000$ & $-0,618034$ \\
24 & $-1,000000$ & $-0,000000$ & $+0,618034$ \\
\hline & & & \\
\hline & & & \\
\hline & & &
\end{tabular}

Tabelle A.2: Koordinaten der symmetrischen Standard Diffusionsrichtungen für DWSSSTEAM im raumfesten Bezugssystem des NMR-Scanners, entsprechend den Flächennormalen zweier gegeneinander gedrehter Dodekaeder (siehe Abbildung 3.10). 


\section{Symbolverzeichnis}

$\alpha, \alpha(n)$

b

$\Delta$

$\Delta_{\mathrm{D}}$

$\delta_{\mathrm{D}}$

D, $D$

$\mathbf{D}_{\text {eff }}$

$\gamma$

H

$\hbar$

$(\cdot)^{*}$

i

$\boldsymbol{k}(t)$

$\langle\cdot\rangle$

$\mathcal{F}\{f\}(x)$

$\mathcal{P}\left(\boldsymbol{r} \mid \boldsymbol{r}_{0}, \Delta_{\mathrm{D}}\right)$

$\mathcal{P}_{G}(\phi, t)$
Auslenkungswinkel der Magnetisierung durch einen RF-Puls bzw. Winkelfunktion einer Abfolge von Anregungspulsen in Abhängigkeit von deren Index $n$, Seite 8

b-Matrix, Gleichung (2.26), Seite 17

Differenzoperator, Seite 5

zeitlicher Abstand eines Paares diffusionskodierender Pulse, Seite 14

Dauer eines diffusionskodierenden Pulses, Seite 14

Diffusionstensor und Diffusionskonstante, Seite 14

effektiver Diffusionstensor, Seite 15

gyromagnetisches Verhältnis, Seite 4

Hamilton-Operator, Seite 4

Plancksches Wirkungsquantum, Seite 4

Hermitesche Konjugation des durch Klammern angedeuteten Ausdrucks, Seite 9

imaginäre Einheit, Seite 8

k-Raum-Vektor zum Zeitpunkt $t$ während des Durchfahrens einer k-Raum-Trajektorie; siehe Formel (2.23), Seite 17

Erwartungswert bzw. Ensemblemittel, Seite 5

Fourier-Transformation der Funktion $f(k)$, Seite 24

Diffusionspropagator, Seite 19

zeitabhängige Phasenverteilung des Spinsystems bei Gradientenschaltung $\boldsymbol{G}(t)$, Seite 14 
$M_{\perp}$

$a$

$v$

$\mu$

B

$\boldsymbol{B}_{0}$

$B_{1}$

$B_{\text {eff }}$

$\boldsymbol{B}_{\mathrm{G}}$

G

$J$

j

M

$\boldsymbol{v}_{n}$

$N_{\text {av }}$

$N_{\mathrm{b}}$

$N_{\mathrm{G}}$

$\omega_{0}$

$\omega_{1}$

$\phi$

$\mathcal{R}$

$\rho(\boldsymbol{r}, t)$

$r$ (komplexe Darstellung der) Transversalkomponente der Magnetisierung in der $x$ - $y$-Ebene, die gestrichene Variante $M_{\perp}^{\prime}$ bezieht sich auf das rotierende Bezugsystem, Seite 8

Vektor in einem linearen überbestimmten Gleichungssystem, dessen Dimension der Anzahl der Gleichungen entspricht, siehe z. B. Gleichung (3.3), Seite 25

aufzulösender Variablenvektor eines überbestimmten Gleichungssystems (siehe Abschnitte 3.1 und 4.1), Seite 25

atomares magnetisches Moment, Seite 4

der aus der Addition aller makroskopischen Magnetfelder resultierende Vektor, Seite 6

statisches, homogenes externes Magnetfeld, Seite 4

externes magnetisches Wechselfeld mit Trägerfrequenz $\omega_{1}$, Seite 4

effektives Magnetfeld bei RF-Anregung im rotierten Bezugssystem, Seite 8

magnetisches Gradientenfeld, Seite 11

Gradient der z-Komponente eines Magnetfeldes, Seite 11

Drehimpuls bzw. Drehimpulsoperator mit Quantenzahlen $j$ und $m$, Seite 4

Teilchenstromdichte, Seite 15

makroskopische Magnetisierung eines Spin-Ensembles, Seite 5

Propagationsrichtung eines Traktographiealgorithmus' im $n$-ten Schritt, Seite 78

Anzahl der Mittelungen eines Bildes, Seite 34

Anzahl der von Null verschiedenen gemessenen b-Werte, Seite 25

Anzahl der gemessenen Diffusionsrichtungen, Seite 25

Larmorfrequenz, Seite 4

Frequenz des Wechselfeldes $\boldsymbol{B}_{1}$, Seite 4

Phase einer Isochromate, Seite 12

Residuum eines Ausgleichsproblems, Seite 26

orts- und zeitabhängige Teilchendichte, Seite 15

Ortsvektor, Seite 11 
Standardabweichung, Seite 26

Kovarianzmatrix der Rauschterme von Einzelmessungen, Seite 26

Echozeit, Seite 8

Anregungsdauer eines RF-Pulses, Seite 7

Dauer der Puls-Schaltung der DW-SSSTEAM-Sequenz für die Akquisition einer Schicht, Seite 39

Dauer der Puls-Schaltung im Ausleseteil der DW-SSSTEAM-Sequenz für die Akquisition einer k-Raum-Linie, Seite 39

Spur eines Operators, Seite 17

Transposition eines Tensors, Seite 16

in $x$-Richtung fouriertransformiertes Signal der $l$-ten Spule eines Phased Arrays, Seite 52

Menge der Eigenwerte (Hauptdiffusivitäten) eines Diffusionstensors, $i \in\{1,2,3\}$, Seite 27

Menge der Eigenvektoren eines Diffusionstensors, $i \in\{1,2,3\}$, Seite 27

Einheitsvektoren eines Koordinatensystems, dessen $z$-Achse in $\boldsymbol{B}_{0^{-}}$ Richtung zeigt, Seite 4

mit der Anregungsfrequenz $\omega_{1}$ um $\boldsymbol{e}_{z}$ rotierendes Koordinatensystem, Seite 7

Diffusionsabfall in einem Stejskal-Tanner-Experiment mit Gradientenschaltung $\boldsymbol{G}(t)$, Seite 14

b-Wert, Diffusionswichtung (2.27), Seite 17

Einhüllende des anregenden Magnetfeldes $\boldsymbol{B}_{1}$, Seite 7

Empfangscharakteristik bzw. Sensitivitätsprofil der $l$-ten Spule eines Phased Arrays. Element $E_{l \rho}$ der Sensitivitätsmatrix E, Seite 49

gemessene Bildintensität bzw. Graustufenwert in Anwesenheit von Rauschen, Seite 34

Boltzmann-Konstante, Seite 6

Gleichgewichtsmagnetisierung eines Systems in einem homogenen Magnetfeld $\boldsymbol{B}_{0}$, Seite 6

Wahrscheinlichkeitsverteilung der Intensitätswerte eines Bildpunktes, Seite 35 
$R$

$S(t)$

$t_{-}, t_{+}$

$T_{1}$

$T_{2}$

$T_{2}^{\star}$

$w_{l}^{(m)}(x)$

$\mathrm{ADC}$

FA

FOV

FWHM

$c_{1}$

$c_{\mathrm{p}}$

$c_{\mathrm{S}}$

$N_{\text {ref }}$

$T M$
Reduktionsfaktor bei PPI (ab Kapitel 4), typische Länge (Kapitel 2), Seite 14 gemessenes NMR-Signal, Seite 14

Zeitpunkt direkt vor (-) bzw. nach (+) einer RF-Anregung, Seite 8

Spin-Gitter-Relaxationszeit, Seite 6

Spin-Spin-Relaxationszeit, Seite 6

effektive Spin-Spin-Relaxationszeit, Seite 7

Gewicht der $l$-ten Spule beim Fitten von Sensitivitätsprofilen an harmonische Funktionen der Ordnung $m$ bei SMASH, Seite 52

Apparent Diffusion Coefficient, siehe Gleichung (3.9), Seite 27

Fraktionelle Anisotropie, siehe Gleichung (3.10), Seite 27

Field of View, siehe Gleichung (2.48), Seite 23

Halbwertsbreite einer unimodalen, symmetrischen Verteilung, Seite 41

Linearitätsindex, siehe Gleichung (3.11a), Seite 29

Planaritätsindex, siehe Gleichung (3.11c), Seite 29

Spherizitätsindex, siehe Gleichung (3.11b), Seite 29

Anzahl der Referenzlinien zur Bestimmung des Sensitivitätsprofils einer Spule, siehe Abbildung 4.1, Seite 50

Länge des Mittenintervalls zwischen dem zweiten und dritten $90^{\circ}$ Puls einer STEAM-Sequenz, Seite 39 


\title{
Abkürzungsverzeichnis
}

\author{
ADC $\ldots . . \ldots \ldots \ldots \ldots$ Apparent Diffusion Coefficient \\ AKS ............. Autokalibrierungssignal \\ CA $\ldots \ldots \ldots \ldots \ldots$ Commissura anterior \\ CC............... Corpus callosum \\ CG ............... Cingulum \\ CGL .............. Corpus geniculatum laterale \\ CS ................ Chemical Shift \\ CW ................ Continuous Wave \\ DeffCoN ........... effective Diffusion Coefficient Navigator \\ DICOM ............ Medizinisches Bildformat \\ DTI.............. Diffusion Tensor Imaging \\ DW-SSSTEAM ...... Diffusion-Weighted Single-Shot Stimulated Echo Acquisition \\ Mode \\ EKG............... Elektrokardiogramm \\ EPI $\ldots \ldots \ldots \ldots \ldots \ldots$ Echo Planar Imaging \\ FA ............... Fraktionelle Anisotropie \\ FACT $\ldots \ldots \ldots \ldots \ldots$ Fiber Assignment by Continuous Tracking \\ FF $\ldots \ldots \ldots \ldots \ldots \ldots$ Full Fourier
}


FFP

Fibrae frontopontinae

FFT Fast Fourier Transform

FID Free Induction Decay

FL Fasciculus longitudinalis

FLI Fasciculus longitudinalis inferior

FLS Fasciculus longitudinalis superior

FMRI functional Magnetic Resonance Imaging

FOF Fasciculus occipitofrontalis (inferior)

FOFS Fasciculus occipitofrontalis superior

FOPP Fibrae occipito- und parietopontinae

FOV Field Of View

FSE Fast Spin-Echo

FU Fasciculus uncinatus

FWHM Full Width at Half Maximum

FX Fornix

GE.

Gradienten-Echo

GPL GNU Public License

GRAPPA Generalized Autocalibrated Partially Parallel Acquisitions

${ }^{1} \mathrm{H}$ Wasserstoff-Kern

$\mathrm{HF}$ Half Fourier

IDEA Integrated Development Environment for Applications

$\mathrm{LM}$ Lemniscus medialis

MDD Main Diffusion Direction

MRI Magnetic Resonance Imaging

MRINF . Musculus rectus inferior

MRL Musculus rectus lateralis 
MRM Musculus rectus medialis

MRSUP Musculus rectus superior

NMR Nuclear Magnetic Resonance

$\mathrm{NO}$ Nervus opticus

PCI . Pedunculus cerebellaris inferior

PCM-D Pedunculus cerebellaris medius (dorsal)

PCM-V . . Pedunculus cerebellaris medius (ventral)

PCS Pedunculus cerebellaris superior

$\mathrm{PF}$ Partial Fourier

PGSE Pulsed-Gradient Spin-Echo

PNS Periphäres Nervensystem

POCS Projection Onto Convex Subsets

POF . Perpendicular occipital fasciculus

PPI Partially Parallel Imaging mit externer (PPI-e) und interner (PPI-i) Referenz

PSF Point Spread Function

RAT Radiatio anterior thalami

$\mathrm{RF}$ Radiofrequenz

$\mathrm{RO}$ Radiatio optica

ROI. Region Of Interest

SE Spin-Echo

SENSE SENSitivity Encoding

SGP Short Gradient Pulse

SMASH Simultaneous Acquisition of Spatial Harmonics

SNR. Signal-to-Noise Ratio

SOS Sum-Of-Squares Reconstruction

STE Stimuliertes Echo 
SVD .............. Singular Value Decomposition

TCS .............. Tractus corticospinalis

TO $\ldots \ldots \ldots \ldots \ldots$ Tractus opticus

TST $\ldots \ldots \ldots \ldots \ldots$ Tractus spinothalamicus

VD-AUTO-SMASH .. Variable Density Auto-Calibrated SMASH

ZNS ............. Zentrales Nervensystem 


\section{Literaturverzeichnis}

[1] Rabi, I. I., S. Millman, P. Kusch und J. R. Zacharias: The Molecular Beam Resonance Method for Measuring Nuclear Magnetic Moments. Phys. Rev., 55:526535 (1939).

[2] Bloch, F.: Nuclear Induction. Phys. Rev., 70:460-474 (1946).

[3] Bloch, F., W. W. Hansen und M. Packard: The Nuclear Induction Experiment. Phys. Rev., 70:474-485 (1946).

[4] Purcell, E. M., H. C. Torrey und R. V. Pound: Resonance Absorption by Nuclear Magnetic Moments in a Solid. Phys. Rev., 69:37-38 (1946).

[5] Bloembergen, N., E. M. Purcell und R. V. Pound: Relaxation Effects in Nuclear Magnetic Resonance Absorption. Phys. Rev., 73:679-712 (1948).

[6] Hahn, E. L.: Spin-Echos. Phys. Rev., 80:580-594 (1950).

[7] Lauterbur, P. C.: Image formation by induced local interactions: Examples employing nuclear magnetic resonance. Nature, 242:190-191 (1973).

[8] Mansfield, P. und P. K. Grannell: NMR Diffraction in Solids? J. Phys. C, 6:L422-L426 (1973).

[9] Cooley, J. W. und J. W. Tukey: An algorithm for the machine calculation of complex Fourier series. Math. Computation, 19:297-301 (1965).

[10] Haase, A., J. Frahm, D. Matthaei, K. D. Merboldt und W. Hänicke: FLASH imaging: Rapid NMR imaging using low flip angle pulses. J. Magn. Reson., 67:258-266 (1986).

[11] Mansfield, P.: Multi-planar image formation using NMR spin echoes. J. Phys. C, 10:L55-L58 (1977). 
[12] Tanner, J. E.: Self diffusion of water in frog muscle. Biophys. J., 28:107-116 (1979).

[13] Stejskal, E. O. und J. E. Tanner: Spin Diffusion Measurements: Spin Echoes in the Presence of a Time-Dependent Field Gradient. J. Chem. Phys., 288-292 (1965).

[14] Basser, P., J. Mattiello und D. LeBihan: MR Diffusion Tensor Spectroscopy and Imaging. Biophys. J., 66:259-267 (1994).

[15] Basser, P., J. Mattiello und D. LeBihan: Estimation of the Effective SelfDiffusion Tensor from the NMR Spin Echo. J. Magn. Reson. B, 103:247-254 (1994).

[16] Woessner, D. E.: Effects of Diffusion in Nuclear Magnetic Resonance Spin-Echo Experiments. J. Chem. Phys., 34:2057-2061 (1961).

[17] Liang, Z.-P. und P. C. Lauterbur: Principles of Magnetic Resonance Imaging: A Signal Processing Perspective (IEEE Press, New York, 2000).

[18] Price, W. S.: Pulsed-Field Gradient Nuclear Magnetic Resonance as a Tool for Studying Translational Diffusion: Part 1. Basic Theory. Concepts Magn. Reson., 299-336 (1997).

[19] Price, W. S.: Pulsed-Field Gradient Nuclear Magnetic Resonance as a Tool for Studying Translational Diffusion: Part II. Experimental Aspects. Concepts Magn. Reson., 10:197-237 (1998).

[20] Torrey, H. C.: Bloch Equation with Diffusion Terms. Phys. Rev., 104:563-565 (1956).

[21] Stejskal, E. O.: Use Of Spin Echoes in a Pulsed Magnetic Field Gradient to Study Anisotropic Restricted Diffusion and Flow. J. Chem. Phys., 3597-3603 (1965).

[22] LeBihan, D. (Hrsg.): Diffusion and Perfusion Magnetic Resonance (Raven Press, 1995).

[23] Basser, P. J. und D. K. Jones: Diffusion-tensor MRI: theory, experimental design and data analysis — a technical review. NMR Biomed., 15:456-467 (2002).

[24] Kärger, J. und W. Heink: The Propagator Representation of Molecular Transport in Microporous Crystallites. J. Magn. Reson., 51:1-7 (1983).

[25] Cory, D. G. und A. N. Garroway: Measurement of Translational Displacement Probabilities by NMR: An Indicator of Compartmentation. Magn. Reson. Med., 14:435-444 (1990). 
[26] Callaghan, P. T., C. D. Eccles und Y. XiA: NMR microscopy of dynamic displacements: k-space and q-space imaging. J. Phys. E: Sci. Instrum., 21:820-822 (1988).

[27] Basser, P. J.: Relationships Between Diffusion Tensor and q-Space MRI. Magn. Reson. Med., 47:392-397 (2002).

[28] Jansons, K. M. und D. C. Alexander: Persistent angular structure: new insights from diffusion magnetic resonance imaging data. Inverse Problems, 19:10311046 (2003).

[29] Tuch, D. S.: Q-Ball Imaging. Magn. Reson. Med., 52:1358-1372 (2004).

[30] Onsager, L.: Reciprocal Relations in Irreversible Processes. I. Phys. Rev., 37:405426 (1931).

[31] Onsager, L.: Reciprocal Relations in Irreversible Processes. II. Phys. Rev., 38:2265-2279 (1931).

[32] Deuflhard, P. und A. Hohmann: Numerische Mathematik (Walter de Gruyter, 1991).

[33] Pierpaoli, C. und P. J. BAsser: Toward a Quantitative Assessment of Diffusion Anisotropy. Magn. Reson. Med., 36:893-906 (1996).

[34] Basser, P. J. und C. Pierpaoli: Microstructural and Physiological Features of Tissues Elucidated by Quantitative-Diffusion-Tensor MRI. J. Magn. Reson. B, 111:209-219 (1996).

[35] Pajevic, S. und P. Basser: Parametric and non-parametric statistical analysis of DT-MRI data. J. Magn. Reson., 161:1-14 (2003).

[36] Kingsley, P. B. und W. G. Monahan: Contrast-to-Noise Ratios of Diffusion Anisotropy Indices. Magn. Reson. Med., 53:911-918 (2005).

[37] Westin, C.-F., S. E. Maier, H. Mamata, A. Navabi, F. A. Jolesz und R. KIKINIS: Processing and visualization for diffusion tensor MRI. Medical Image Analysis, 6:93-108 (2002).

[38] Clark, C. A., M. Hedehus und M. E. Moseley: Diffusion Time Dependence of the Apparent Diffusion Tensor in Healthy Human Brain and White Matter Disease. Magn. Reson. Med., 45:1126-1129 (2001).

[39] Bernstein, M. A., K. F. King und X. J. Zhou: Handbook of MRI Pulse Sequences (Academic Press, 2004).

[40] Enzmann, D. R. und N. J. PelC: Brain motion: measurement with phasecontrast MR imaging. Radiology, 185:653-660 (1992). 
[41] Pierpaoli, C., S. Marenco, G. Rohde, D. K. Jones und A. S. Barnett: Analyzing the contribution of cardiac pulsation to the variability of quantities derived from the diffusion tensor. In: Proc. Intl. Soc. Mag. Reson. Med. (2003), Bd. 11, 70 .

[42] Skare, S. und J. L. R. Andersson: On the effects of gating in diffusion imaging of the brain using single shot EPI. Magn. Reson. Imaging, 19:1125-1128 (2001).

[43] Chang, L. C., D. K. Jones und C. Pierpaoli: ReSTORE: Robust Estimation of Tensors by Outlier Rejection. Magn. Reson. Med., 53:1088-1095 (2005).

[44] Sun, P. Z., J. G. Seland und D. Cory: Background gradient suppression in pulsed gradient stimulated echo measurements. J. Magn. Reson., 161:168-173 (2003).

[45] Neeman, M., J. P. Freyer und L. O. Sillerud: A Simple Method for Obtaining Cross-Term-Free Images for Diffusion Anisotropy Studies in NMR Microimaging. Magn. Reson. Med., 21:138-143 (1991).

[46] Wehrli, F. W.: Magnetic Resonance Imaging: Principles of Magnetic Resonance, Bd. I (Mosby Year Book, 1992), 2. Aufl..

[47] Gudbuartsson, H. und S. Patz: The Rician Distribution of Noisy MRI Data. Magn. Reson. Med., 34:910-914 (1995).

[48] Dietrich, O., S. Heiland und K. Sartor: Noise Correction for the Exact Determination of Apparent Diffusion Coefficients at Low SNR. Magn. Reson. Med., 45:448-453 (2001).

[49] Anderson, A. W.: Theoretical Analysis of the Effects of Noise on Diffusion Tensor Imaging. Magn. Reson. Med., 46:1174-1188 (2001).

[50] Jones, D. K. und P. J. BAsser: Squashing Peanuts and Smashing Pumpkins:How Noise Distorts Diffusion-Weighted MR Data. Magn. Reson. Med., 57:979-993 (2004).

[51] Batchelor, P. G., D. Atkinson, D. L. G. Hill, F. Calamante und A. Connelly: Anisotropic Noise Propagation in Diffusion Tensor MRI Sampling Schemes. Magn. Reson. Med., 49:1143-1151 (2003).

[52] Perona, P. und J. Malik: Scale-space and edge detection using anisotropic diffusion. IEEE Transactions on Pattern Analysis and Machine Intelligence, 12:629-639 (1990).

[53] Bastin, M. E. und P. A. Armitage: Utilizing the Diffusion-to-Noise Ratio to Optimize Magnetic Resonance Diffusion Tensor Acquisition Strategies for Improving Measurements of Diffusion Anisotropy. Magn. Reson. Med., 45:1056-1065 (2001). 
[54] Jones, D. K., S. C. R. Williams, D. Gasston, M. A. Horsfield, A. SimMONS und R. HowARD: Isotropic Resolution Diffusion Tensor Imaging With Whole Brain Acquisition in a Clinically Acceptable Time. Human Brain Mapping, 15:216-230 (2002).

[55] Brihuega-Moreno, O., F. P. Heese und L. D. Hall: Optimization of Diffusion Measurements Using Cramer-Rao Lower Bound Theory and Its Application to Articular Cartilage. Magn. Reson. Med., 50:1069-1076 (2003).

[56] Jones, D. K.: The Effect of Gradient Sampling Schemes on Measures Derived From Diffusion Tensor MRI: a Monte Carlo Study. Magn. Reson. Med., 51:807815 (2004).

[57] Skare, S., M. Hedehus, M. E. Moseley und T.-Q. Li: Condition Number as a Measure of Noise Performance of Diffusion Tensor Data Acquisition Schemes with MRI. J. Magn. Reson., 147:340-352 (2000).

[58] Jones, D. K., M. A. Horsfield und A. Simmons: Optimal Strategies for Measuring Diffusion in Anisotropic Systems by Magnetic Resonance Imaging. Magn. Reson. Med., 42:515-525 (1999).

[59] Frahm, J., A. HaAse, D. Matthaei, K. D. Merboldt und W. Hänicke: Rapid NMR imaging using stimulated echoes. J. Magn. Reson., 65:130-135 (1985).

[60] Nolte, U. G.: Diffusionskodierende NMR-Tomografie des Gehirns. Diplomarbeit, Universität Göttingen (1999).

[61] Nolte, G. U., J. Finsterbusch und J. Frahm: Rapid Isotropic Diffusion Mapping Without Susceptibility Artifacts: Whole Brain Studies Using DiffusionWeighted Single-Shot STEAM MR Imaging. Magn. Reson. Med., 44:731-736 (2000).

[62] Jaermann, T., K. P. Pruessmann, A. Valvanis, S. Kollias und P. BoeSIGER: Influence of SENSE on Image Properties in High-Resolution Single-Shot Echo-Planar DTI. Magn. Reson. Med., 55:335-342 (2006).

[63] Thomas, D. L., G. S. Pell, M. F. Lythgoe, D. G. Gadian und R. J. ORDIDGE: A Quantitative Method for Fast Diffusion Imaging Using MagnetizationPrepared TurboFLASH. Magn. Reson. Med., 39:950-960 (1998).

[64] Wang, F.-N., T.-Y. Huang, F.-H. Lin, T.-C. Chuang, N.-K. Chen, H.-W. Chung, C.-Y. Chen und K. K. Kwong: PROPELLER EPI: An MRI Technique Suitable for Diffusion Tensor Imaging at High Field Strength With Reduced Geometric Distortions. Magn. Reson. Med., 54:1232-1240 (2005).

[65] Pipe, J. G., V. G. Farthing und K. P. Forbes: Multishot Diffusion-Weighted FSE Using PROPELLER MRI. Magn. Reson. Med., 47:42-52 (2002). 
[66] PIPE, J. G.: Turboprop - an improved PROPELLER sequence for diffusionweighted MRI. In: Proc. Intl. Soc. Mag. Reson. Med. (2002), Bd. 10, 435.

[67] Mamata, H., Y. Mamata, C.-F. Westin, M. Shenton, R. Kikinis, F. A. JoLESZ und S. E. MAIER: High-Resolution Line Scan Diffusion Tensor MR Imaging of White Matter Fiber Tract Anatomy. Am. J. Neuroradiol., 23:67-75 (2002).

[68] Li, T.-Q., D.-H. Kim und M. E. Moseley: High-Resolution Diffusion-Weighted Imaging With Interleaved Variable-Density Spiral Acquisitions. J. Magn. Reson. Imaging, 21:468-475 (2005).

[69] Jeong, E.-K., S.-E. Kim und D. L. PARker: High-Resolution DiffusionWeighted 3D MRI, Using Diffusion-Weighted Driven-Equilibrium (DW-DE) and Multishot Segmented 3D-SSFP Without Navigator Echoes. Magn. Reson. Med., 50:821-829 (2003).

[70] Golay, X., H. Jiang, P. C. M. van ZiJl und S. Mori: High-Resolution Isotropic 3D Diffusion Tensor Imaging of the Human Brain. Magn. Reson. Med., 47:837-843 (2002).

[71] Callaghan, P. T.: Principles of Nuclear Magnetic Resonance Microscopy (Oxford University Press, 1991).

[72] Finsterbusch, J. und J. Frahm: Half-Fourier Single-Shot STEAM MRI. Magn. Reson. Med., 47:611-615 (2002).

[73] Rieseberg, S., K. D. Merboldt, M. Küntzel und J. Frahm: Diffusion Tensor Imaging using Partial Fourier STEAM MRI with Projection onto Convex Subsets Reconstruction. Magn. Reson. Med., 54:486-490 (2005).

[74] Rieseberg, S.: Kartierung des Diffusionstensors mittels schneller NMRTomographie. Axonale Konnektivität im menschlichen Gehirn. Dissertation, Universität Göttingen (2004).

[75] Cuppen, J. J. und A. van Est: Reducing MR imaging time by one-sided reconstruction. In: Topical Conference on Fast MRI Techniques (1987).

[76] Roemer, P. B., W. A. Edelstein, C. E. Hayes, S. P. Souza und O. M. Mueller: The NMR phased array. Magn. Reson. Med., 16:192-225 (1990).

[77] Larsson, E. G., D. Erdogmus, R. Yan, J. C. Principe und J. R. FitzsimMONS: SNR-optimality of sum-of-squares reconstruction for phased-array magnetic resonance imaging. J. Magn. Reson., 163:121-123 (2003).

[78] Sodickson, D. K. und W. J. Manning: Simultaneous Acquisition of Spatial Harmonics (SMASH): Fast Imaging with Radiofrequency Coil Arrays. Magn. Reson. Med., 38:591-603 (1997). 
[79] Pruessmann, K. P., M. Weiger, M. B. Scheidegger und P. Boesiger: SENSE: Sensitivity Encoding for Fast MRI. Magn. Reson. Med., 42:952-962 (1999).

[80] RA, J. B. und C. Y. Rim: Fast Imaging Using Subencoding Data Sets from Multiple Detectors. Magn. Reson. Med., 30:142-145 (1993).

[81] Sodickson, D. K., M. A. Griswold, P. M. Jakob, R. R. Edelman und W. J. Manning: Signal-to-Noise Ratio and Signal-to-Noise Efficiency in SMASH imaging. Magn. Reson. Med., 41:1009-1022 (1999).

[82] Jakob, P. M., M. A. Griswold, R. R. Edelman und D. K. Sodickson: AUTO-SMASH: a self-calibrating technique for SMASH imaging. MAGMA, 7:5254 (1998).

[83] Heidemann, R. M., M. A. Griswold, A. Haase und P. M. Jakob: VDAUTO-SMASH Imaging. Magn. Reson. Med., 45:1066-1074 (2001).

[84] Griswold, M. A., P. M. Jakob, R. M. Heidemann, M. Nittka, V. JelLus, J. Wang, B. Kiefer und A. HaAse: Generalized Autocalibrating Partially Parallel Acquisitions (GRAPPA). Magn. Reson. Med., 47:1202-1210 (2002).

[85] Lu, H., L. M. Nagae-Poetscher, X. Golay, D. Lin, M. Pomper und P. C. M. VAN ZIJL: Routine Clinical Brain MRI Sequences for Use at 3.0 Tesla. J. Magn. Reson. Imaging, 22:13-22 (2005).

[86] Jiang, H., X. Golay, P. C. M. van ZiJl und S. Mori: Origin and Minimization of Residual Motion-Related Artifacts in Navigator-Corrected Segmented DiffusionWeighted EPI of the Human Brain. Magn. Reson. Med., 47:818-822 (2002).

[87] Finsterbusch, J. und M. A. Koch: (Almost) Free Lunch: Single-Shot STEAM MRI with GRAPPA. In: Proc. Intl. Soc. Mag. Reson. Med. (2003), Bd. 11, 1012.

[88] Finsterbusch, J. und M. A. Koch: Single-Shot STEAM MRI combined with SENSE. In: Proc. Intl. Soc. Mag. Reson. Med. (2004), Bd. 12, 2251.

[89] Koay, C. G., J. D. Carew, A. L. Alexander, P. J. Basser und M. E. MEYERAND: Investigation of Anomalous Estimates of Tensor-Derived Quantities in Diffusion Tensor Imaging. Magn. Reson. Med., 55:930-936 (2006).

[90] Ogawa, S., D. Tank, R. Menon, J. Ellermann, S. Kim, H. Merkle und K. UGURBIL: Intrinsic signal changes accompanying sensory stimulation - functional brain mapping with magnetic-resonance-imaging. Proc. Natl. Acad. Sci. U.S.A., 89:5951-5955 (1992).

[91] Biswal, B. B., F. Z. Yetkin, V. M. Haughton und J. S. Hyde: Functional connectivity in the motor cortex of resting human brain using echo-planar MRI. Magn. Reson. Med., 34:537-541 (1995). 
[92] Dejerine, J.: Anatomie des Centres Nerveux (Rueff, 1895).

[93] Vogt, O.: Neurobiologische Arbeiten. Erste Serie: Beiträge zur Hirnfaserlehre (Fischer, 1902).

[94] Axer, H., M. Axer, T. Krings und D. G. von Keyserlingk: Quantitative estimation of $3 D$ fiber course in gross histological sections of the human brain. J. Neurosci. Methods, 105:121-131 (2001).

[95] KlingleR, J.: Erleichterung der makroskopischen Präparation des Gehirns durch den Gefrierprozeß. Schweiz. Arch. Neurol., 36:247-256 (1935).

[96] Pierpaoli, C., A. Barnett, S. Pajevic, R. Chen, L. Penix, A. Virta und P. BASSER: Water Diffusion Changes in Wallerian Degeneration and Their Dependence on White Matter Architecture. NeuroImage, 13:1174-1185 (2001).

[97] Conturo, T. E., N. F. Lori, T. S. Cull, E. Akbudak, A. Z. Snyder, J. S. Shimony, R. C. McKinstry, H. Burton und M. E. Raichle: Tracking neuronal fiber pathways in the living human brain. Proc. Natl. Acad. Sci. USA, 96:10422-10427 (1999).

[98] Koch, M. A., D. G. Norris und M. Hund-Georgiadis: An investigation of functional and anatomical connectivity using magnetic resonance imaging. NeuroImage, 16:241-250 (2002).

[99] Behrens, T. E. J., M. W. Woolrich, M. Jenkinson, H. Johansen-Berg, R. G. Nunes, S. Clare, P. M. Matthews, J. M. Brady und S. M. Smith: Characterization and Propagation of Uncertainty in Diffusion-Weighted MR Imaging. Magn. Reson. Med., 50:1077-1088 (2003).

[100] Basser, P. J., S. Pajevic, C. Pierpaoli, J. Duda und A. Aldroubi: In Vivo Fiber Tractography Using DT-MRI Data. Magn. Reson. Med., 44:625-632 (2000).

[101] Mori, S., B. Crain, V. Chacko und P. C. M. van ZiJl: Three-Dimensional Tracking of Axonal Projections in the Brain by Magnetic Resonance Imaging. Ann. Neurol., 45:265-269 (1999).

[102] Catani, M., R. J. Howard, S. Pajevic und D. K. Jones: Virtual in Vivo Interactive Dissection of White Matter Fasciculi in the Human Brain. NeuroImage, 17:77-94 (2001).

[103] Weinstein, D., G. Kindlmann und E. Lundberg: Tensorlines: AdvectionDiffusion based Propagation through Diffusion Tensor Fields. In: IEEE Visualization Proc., San Francisco (1999), 249-252.

[104] Lazar, M., J. H. Lee und A. L. Alexander: Axial Asymmetry of Water Diffusion in Brain White Matter. Magn. Reson. Med., 54:860-867 (2005). 
[105] GSL: GNU Scientific Library. http://www.gnu.org/software/gsl/.

[106] BLAS: Basic Linear Algebra Subprograms. http://www.netlib.org/blas/.

[107] ATLAS: Automatically Tuned Linear Algebra Software. http://math-atlas. sourceforge.net/.

[108] LAPACK: Linear Algebra Package. http://www.netlib.org/lapack/.

[109] Qt. http://www.trolltech.no/products/qt.

[110] VTK: The Visualization Toolkit. http://www.vtk.org.

[111] ITK: The Insight Toolkit. http://www.itk.org.

[112] Crosby, E. C., T. Humphrey und E. W. Lauer: Correlative Anatomy of the Nervous System (Macmillan, 1962).

[113] Nieuwenhuys, R., J. Voogd und C. V. Huijzen: The Human Central Nervous System (Springer, 1981).

[114] Trepel, M.: Neuroanatomie: Struktur und Funktion (Urban \& Fischer, 1999).

[115] Catani, M., D. K. Jones, R. Donato und D. Ffytche: Occipito-temporal connections in the human brain. Brain, 126:2093-2107 (2003).

[116] Wakana, S., H. Jiang, L. M. Nagae-Poetscher, P. C. M. van ZiJl und S. MorI: Fiber Tract-based Atlas of Human White Matter Anatomy. Radiology, 230:77-87 (2004).

[117] Stieltues, B., W. E. Kaufmann, P. C. M. van Zijl, K. Fredericksen, G. D. Pearlson, M. Solaiyappan und S. Mori: Diffusion Tensor Imaging and Axonal Tracking in the Human Brainstem. NeuroImage, 14:723-735 (2001).

[118] Tuch, D. S., T. G. Reese, M. R. Wiegell und J. van Wedeen: Diffusion MRI of Complex Neuronal Architecture. Neuron, 40:885-895 (2003).

[119] Huang, H., J. Zhang, H. Jiang, S. Wakana, L. Poetscher, M. I. MilLER, P. van ZiJl, A. Hillis, R. Wytik und S. Mori: DTI tractography based parcellation of white matter: Application to the mid-sagittal morphology of corpus callosum. NeuroImage, 26:195-205 (2005).

[120] Di Virgilio, G., S. Clarke, G. Pizzolato und T. Schaffner: Cortical regions contributing to the anterior commissure in man. Exp. Brain Res., 124:1-7 (1999).

[121] Jellison, B. J., A. S. Field, J. Medow, M. Lazar, M. S. Salamat und A. L. Alexander: Diffusion Tensor Imaging of Cerebral White Matter: A Pictorial Review of Physics, Fiber Tract Anatomy and Tumor Imaging Patterns. Am. J. Neuroradiol., 25:356-369 (2004). 
[122] Ture, U., M. G. YAsArgil und T. G. PAIT: Is There a Superior Occipitofrontal Fasciculus? A Microsurgical Anatomic Study. Neurosurgery, 40:1226-1232 (1997).

[123] Mori, S., W. E. Kaufmann, C. Davatzikos, B. Stieltues, L. Amodei, K. Frederickson, G. D. Pearlson, E. R. Melhem, M. Solaiyappan, G. V. Raymond, H. W. Moser und P. C. M. van Zisl: Imaging Cortical Association Tracts in the Human Brain Using Diffusion-Tensor-Based Axonal Tracking. Magn. Reson. Med., 47:215-223 (2002).

[124] Toosy, A. T., O. Ciccarelli, G. J. M. Parker, C. A. M. WheelerKingshott, D. H. Miller und A. J. Thompson: Characterizing functionstructure relationships in the human visual system with functional MRI and diffusion tensor imaging. NeuroImage, 21:1452-1463 (2005).

[125] Chabert, S., N. Molko, Y. Cointepas, P. Le Roux und D. Le Bihan: Diffusion Tensor Imaging of the Human Optic Nerve Using a Non-CPMG Fast Spin Echo Sequence. J. Magn. Reson. Imaging, 22:307-310 (2005).

[126] Vinogradov, E., A. Degenhardt, D. Smith, R. Maruqis, T. K. Vartanian, P. Kinkel, S. Maier, D. B. Hackney und R. E. Lenkinski: HighResolution Anatomic, Diffusion Tensor, and Magnetization Transfer Imaging of the Optic Chiasm at 3T. J. Magn. Reson. Imaging, 22:302-306 (2005).

[127] Jones, D. K. und C. Pierpaoli: Contribution of Cardiac Pulsation to Variability of Tractography Results. In: Proc. Intl. Soc. Mag. Reson. Med. (2005), Bd. 13, 222. 


\title{
Auf den Schultern von Giganten
}

\author{
If I have seen a little further, it is by \\ standing on the shoulders of giants. \\ Isaac Newton
}

Für die Möglichkeit zur Anfertigung einer Dissertation und die wissenschaftliche Unterstützung möchte ich mich bei Prof. J. Frahm sehr herzlich bedanken. Unter seiner Ägide entstand die vorliegende Arbeit in der „Biomedizinischen NMR Forschungs GmbH“ am Max-Planck-Institut für biophysikalische Chemie. Herr Prof. H. Hofsäss hat dabei freundlicherweise die Betreuung seitens der physikalischen Fakultät der Universität Göttingen übernommen. Tobias Block war so liebenswürdig, Zeit für das Korrekturlesen einiger Kapitel zu erübrigen.

Ich schätze mich glücklich, daß ich vier bereichernde Jahre in einer interessanten Arbeitsgruppe mit netten Kollegen verbringen durfte. All jenen, die mir bei meinen Anstrengungen, auf eigenen wissenschaftlichen Füßen zu stehen, Beistand geleistet haben, sei an dieser Stelle meine Verbundenheit ausgedrückt. Diejenigen, denen ich dabei manchmal auf die Zehen getreten habe, mögen Nachsicht walten lassen.

Das Max-Planck-Institut verdient ein großes Lob für seine im positiven Sinne nahezu unsichtbare Verwaltung und die stimulierende Forschungsatmosphäre. Außerdem soll an dieser Stelle die Gemeinschaft der Entwickler von „open source“ Computersoftware dankende Erwähnung finden, neben meinen wissenschaftlichen Ahnen ein weiterer Gigant mit vielen breiten Schultern und von unschätzbarem Wert für die vorliegende Arbeit.

Meiner Familie fühle ich mich für Rat und Tat in Angelegenheiten jenseits akademischer Fragestellungen und für die großzügige Unterstützung meines Studiums verpflichtet. Einen indirekten aber fundamentalen Beitrag zum harmonischen Abschluß der Promotionzeit verdanke ich Ирина Дуданова, die meinem Leben eine unerwartete und schöne Wendung gab und mich gelehrt hat, daß man Schultern nicht unbedingt erklimmen muß, sondern sich auch an sie anlehnen kann. 


\section{Lebenslauf}

$\begin{array}{ll}\text { NAme } & \text { Matthias Küntzel } \\ \text { GEBURTSDATUM } & 12.05 .1976 \\ \text { GeBURTSORT } & \text { Hannover } \\ \text { StAATSANGEHÖRIGKEIT } & \text { deutsch }\end{array}$

\section{Werdegang}

- 1982-1995 Schulbesuch in Münster/Nordrhein-Westfalen und Bad Nauheim/Hessen. Erwerb der allgemeinen Hochschulreife (Abitur) am St. Lioba-Gymnasium, Bad Nauheim.

- 1995-1996 Zivildienst in der Kerckhoff-Klinik, Bad Nauheim

- 1996-2001 Studium der Physik an der Universität Göttingen. Diplomarbeit am Institut für theoretische Physik, Arbeitsgruppe Prof. A. Zippelius.

- 2002-2006 Promotionsstudiengang Physik an der Universität Göttingen. Anfertigung einer Dissertation. Betreuung durch Prof. H. Hofsäss, Universität Göttingen. Anleitung durch Prof. J. Frahm, „Biomedizinische NMR Forschungs GmbH“ am Max-Planck-Institut für biophysikalische Chemie. 